\title{
AUG 201964
}

NBS MONOGRAPH 25-SECTION 3

Referencatour the to be take litom the library.

\section{Standard X-ray Diffraction}

\section{Powder Patterns}

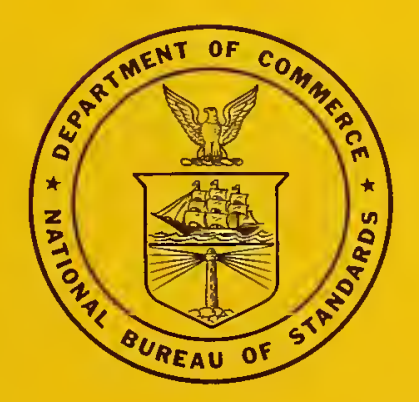

U.S. DEPARTMENT OF COMMERCE

NATIONAL BUREAU OF STANDARDS 


\section{THE NATIONAL BUREAU OF STANDARDS}

The National Bureau of Standards is a principal focal point in the Federal Government for assuring maximum application of the physical and engineering sciences to the advancement of technology in industry and commerce. Its responsibilities include development and maintenance of the national standards of measurement, and the provisions of means for making measurements consistent with those standards; determination of physical constants and properties of materials; development of methods for testing materials, mechanisms, and structures, and making such tests as may be necessary, particularly for government agencies; cooperation in the establishment of standard practices for incorporation in codes and specifications; advisory service to government agencies on scientific and technical problems; invention and development of devices to serve special needs of the government; assistance to industry, business, and consumers in the development and accceptance of commercial standards and simplified trade practice recommendations; administration of programs in cooperation with United States business groups and standards organizations for the development of international standards of practice; and maintenance of a clearinghouse for the collection and dissemination of scientific, technical, and engineering information. The scope of the Bureau's activities is suggested in the following listing of its four Institutes and their organizational units.

Institute for Basic Standards. Electricity. Metrology. Heat. Radiation Physics. Mechanics. Applied Mathematics. Atomic Physics. Physical Chemistry. Laboratory Astrophysics.* Radio Standards Laboratory: Radio Standards Physics; Radio Standards Engineering.** Office of Standard Reference Data.

Institute for Materials Research. Analytical Chemistry. Polymers. Metallurgy. Inorganic Materials. Reactor Radiations. Cryogenics.** Office of Standard Reference Materials.

Central Radio Propagation Laboratory.** Ionosphere Research and Propagation. Troposphere and Space Telecommunications. Radio Systems. Upper Atmosphere and Space Physics.

Institute for Applied Technology. Textiles and Apparel Technology Center. Building Research. Industrial Equipment. Information Technology. Performance Test Development. Instrumentation. Transport Systems. Office of Technical Services. Office of Weights and Measures. Office of Engineering Standards. Office of Industrial Services.

*NBS Group, Joint Institute for Laboratory Astrophysics at the University of Colorado.

**Located at Boulder, Colorado. 
UNITED STATES DEPARTMENT OF COMMERCE • Luther H. Hodges, Secretary NATIONAL BUREAU OF STANDARDS • A. V. Astin, Director

\section{Standard X-ray Diffraction}

\section{Powder Patterns}

Howard E. Swanson, Marlene Cook Morris,

Eloise H. Evans, and Linda Ulmer

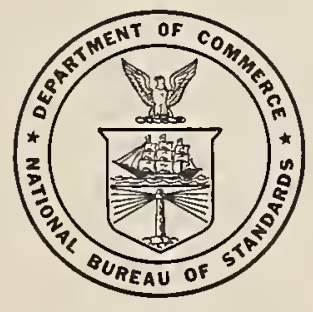

National Bureau of Standards Monograph 25-Section 3

Issued July 31, 1964 
Library of Congress Catalog Card Number: 53-61386 


\section{Contents}

Introduction

Page

Standard x-ray powder patterns:

Aluminum $3: 2$ Silicate (mullite), $3 \mathrm{Al}_{2} \mathrm{O}_{3} \cdot 2 \mathrm{SiO}_{2}$ (orthorhombic)

Ammonium Fluoberyllate, $\left(\mathrm{NH}_{4}\right)_{2} \mathrm{BeF}_{4}$ (ortho-

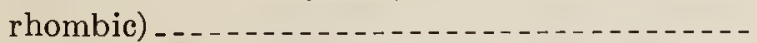

Ammonium Fluoborate, $\mathrm{NH}_{4} \mathrm{BF}_{4}$ (orthorhombic) -

*Antimony Selenide, $\mathrm{Sb}_{2} \mathrm{Se}_{3}$ (orthorhombic) _....-

*Antimony Telluride, $\mathrm{Sb}_{2} \mathrm{Te}_{3}$ (trigonal)

*Arsenic Trioxide, claudetite, $\mathrm{As}_{2} \mathrm{O}_{3}$ (mono-

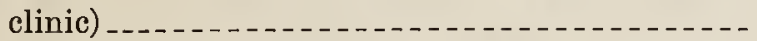

*Barium Bromide Monohydrate, $\mathrm{BaBr}_{2} \cdot \mathrm{H}_{2} \mathrm{O}$ (orthorhombic)

Barium Stannate, $\mathrm{BaSnO}_{3}$ (cubic) ..........

Bismuth Orthophosphate, $\mathrm{BiPO}_{4}$ (monoclinic) -.-

*Bismuth Orthophosphate, $\mathrm{BiPO}_{4}$ (trigonal) -...-

*Bismuth Orthovanadate (low form), $\mathrm{BiVO}_{4}$ (tetragonal)

*Bismuth Orthovanadate (high form) $\mathrm{BiVO}_{4}$

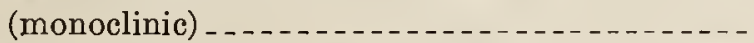

Bismuth Telluride, (tellurobismuthite), $\mathrm{Bi}_{2} \mathrm{Te}_{3}$ (trigonal)

Bismuth Trioxide, alpha (bismite), $\mathrm{Bi}_{2} \mathrm{O}_{3}$ (pseudo-

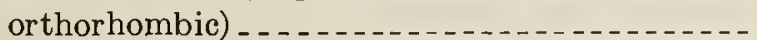

*Cadmium Perchlorate Hexahydrate $\mathrm{Cd}\left(\mathrm{ClO}_{4}\right)_{2}$. $6 \mathrm{H}_{2} \mathrm{O}$ (trigonal)

Cadmium Sulfate, $\mathrm{CdSO}_{4}$ (orthorhombic)

Cadmium Telluride, CdTe (cubic) _............

Calcium Fluoride Phosphate, (fluorapatite),

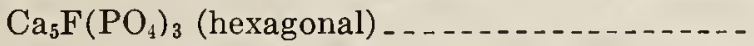

*Cerium Niobium Titanium Oxide, (eschynite), $\mathrm{CeNbTiO}_{6}$ (orthorhombic) _................

*Cesium Chromate, $\mathrm{Cs}_{2} \mathrm{CrO}_{4}$ (orthorhombic) -...Cesium Fluoride, CsF (cubic) _.............

*Cobalt Fluosilicate Hexahydrate, $\mathrm{CoSiF}_{6} \cdot 6 \mathrm{H}_{2} \mathrm{O}$ (trigonal)

*Cobalt Perchlorate Hexahydrate, $\mathrm{Co}\left(\mathrm{ClO}_{4}\right)_{2}$. $6 \mathrm{H}_{2} \mathrm{O}$ (hexagonal)

Copper Sulfate, (chalcocyanite), $\mathrm{CuSO}_{4}$ (ortho-

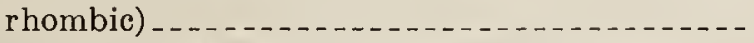

*Dysprosium Arsenate, Dy $\mathrm{AsO}_{4}$ (tetragonal) -.--

*Erbium Arsenate, $\mathrm{ErAsO}_{4}$ (tetragonal) ........
*Europium Arsenate, EuAsO (tetragonal) _..... -

*Gallium Arsenide, GaAs (cubic) ..............

*Holmium Arsenate, $\mathrm{HoAsO}_{4}$ (tetragonal) _....-

Indium Arsenide, In As (cubic) _...........

*Lanthanum Arsenate, $\mathrm{LaAsO}_{4}$ (monoclinic) -...

*Lanthanum Niobium Titanium Oxide, LaNb$\mathrm{TiO}_{6}$ (monoclinic)

Lithium Phosphate, low form, (lithiophosphate),

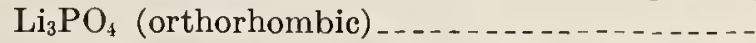

Lithium Phosphate, high form, $\mathrm{Li}_{3} \mathrm{PO}_{4}$ (orthorhombic)

Magnesium Ammonium Phosphate Hexahydrate, (struvite), $\mathrm{MgNH}_{4} \mathrm{PO}_{4} \cdot 6 \mathrm{H}_{2} \mathrm{O}$ (orthorhombic) --

Potassium Chlorate, $\mathrm{KClO}_{3}$ (monoclinic) _.......

*Potassium Lithium Sulfate, $\mathrm{KLiSO}_{4}$ (hexagonal)

*Potassium Perchromate, $\mathrm{K}_{3} \mathrm{CrO}_{8}$ (tetragonal) _ -

*Potassium Zine Decavanadate 16 Hydrate,

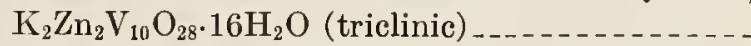
*Rubidium Chromate, $\mathrm{Rb}_{2} \mathrm{CrO}_{4}$ (orthorhombic) -

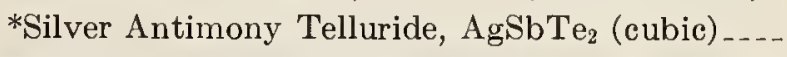
*Sodium Magnesium Aluminum Boron, Hydroxy Silicate, dravite, $\mathrm{NaMg} \mathrm{Ml}_{6} \mathrm{~B}_{3} \mathrm{Si}_{6} \mathrm{O}_{27}(\mathrm{OH})_{4}$ (trig-

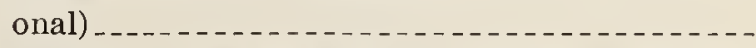

Sodium Trimetaphosphate, $\mathrm{Na}_{3} \mathrm{P}_{3} \mathrm{O}_{9}$ (orthorhombic) _

47

Sodium Trimetaphosphate Monohydrate, $\mathrm{Na}_{3}-$ $\mathrm{P}_{3} \mathrm{O}_{9} \cdot \mathrm{H}_{2} \mathrm{O}$, (orthorhombic)

*Stannous Fluoride, $\mathrm{SnF}_{2}$ (monoclinic) ........*Strontium 1: 1 Borate, $\mathrm{SrO} \cdot \mathrm{B}_{2} \mathrm{O}_{3}$ (orthorhombic) -

*Terbium Arsenate, $\mathrm{TbAsO}_{4}$ (tetragonal) ......*Thallium Chromate, $\mathrm{Tl}_{2} \mathrm{CrO}_{4}$ (orthorhombic)_-.*Thulium Arsenate, $\mathrm{TmAsO}_{4}$ (tetragonal) _.....

Titanium Dioxide brookite, $\mathrm{TiO}_{2}$ (orthorhombic) _

Zinc Telluride, ZnTe (cubic) _................ Cumulative index to Circular 539, Volumes 1, 2, $3,4,5,6,7,8,9$, and 10 , and Monograph 25, Sections 1, 2, and 3

Cumulative mineral index

Page

\section{Errata}

Circular 539

Vol. 1, Page 50, In the pattern of Swanson and Tatge, the $d$-values $0.8576,0.8557$, $0.8467,0.8412$, and 0.8383 should have the following $h k l$ values, 422, 511, $\overline{5} 13,333$, and 206.

Vol. 2, Page 46, Lattice constants paragraph, column 1, line 3: Puma should read Pnma. This change was made in the Vol. 2 reprint.

Vol. 3, Page 54, On the line below hkl 121 insert $h k l$ 012; on the line below $h k l 200$ insert $h k l 031 ; h k l 040$ should read 211 ; delete $h k l$ values 230 and 410 and all those following 421.

Vol. 5, Page 10, Column 1, bottom line, the index of refraction should read 1.710. Page 11, In pattern of Swanson, Gilfrich, and Ugrinic, for $h k l 721 d$ should read 2.078 .

Page 43, Reference No. 3: The fourth author's name should be F. E. Sennett.

Vol. 8, Page 42, Column 1: The density should be 4.697 .

Page 65, $\mathrm{Ti}_{5} \mathrm{Si}_{3}$ should have 2 molecules per unit cell and the density 4.376 .

Vol. 9, Page 6, $\left(\mathrm{NH}_{4}\right)_{2} \mathrm{PtBr}_{6}$ table: $\mathrm{Cu}$ radiation applies to entire table. 


\section{Errata-Continued}

Page 19, hkl 102 should be 012.

Page 47, The density of $\mathrm{K}_{3} \mathrm{ZrF}_{7}$ should be 3.123 .

Monograph 25

Sec. 1, Page 10, Lattice constants table: headings $a b c$ should read $b c a$.

Page 12, The title should read: Erbium Gallium Oxide 3:5. . . .

Sec. 2, Page 1, Column 2 line 11: potassium nitroso chlororhenate should be potassium nitroso chlororuthenate.

Page 2, In the left hand column, the sentence beginning in the seventh line from the bottom should read: Factors for converting integrated intensities to peak height intensities are on the left side of the chart.

Page 8, $\mathrm{CdWO}_{4}$ space group should be $\mathrm{C}_{2 \mathrm{~h}}^{4}-\mathrm{P} 2 / \mathrm{c}$ (No. 13).

Page 11, Spectrographic analysis should read: 0.01 to 0.1 percent sodium and ....

Page 34, The intensity of the line at $d=4.21$ should be 61 . Insert the reading: $h k l=102, d=3.94, I=2$.

Page 35, Refeience No. 7 should read: Helen M. Ondik, to be published in Acta Cryst.

\section{Addenda}

Monograph 25

Sec. 2, Page 22, Insert this data:

\begin{tabular}{|r|r|r|c|}
\hline$h k l$ & $d$ & $I$ & $a$ \\
\hline 930 & 1.2843 & $<1$ & 12.184 \\
965 & 1.0225 & $\sum 1$ & 12.184 \\
10.8 .2 & .9398 & & 12.181 \\
13.5 .0 & .8408 & $\sum 1$ & 12.184 \\
14.3 .3 & .8329 & $<1$ & 12.184 \\
\hline
\end{tabular}

Sec. 2, Page 35, Add this data: The sample is optically negative and the indices of refraction are $\mathrm{N}_{\alpha}=1.440, \mathrm{~N}_{\beta}=1.458$, $\mathrm{N}_{\gamma}=1.474$, and $2 \mathrm{~V} \cong 90^{\circ}$.

\section{Standard X-ray Diffraction Powder Patterns}

The eleven previous volumes in this series are available from the Superintendent of Documents, U.S. Government Printing Office, Washington 25, D.C., as follows:

NBS Circular 539, Volume 1, Standard X-ray Diffraction Powder Patterns (Data for 54 inorganic substances) 45 cents

NBS Circular 539, Volume 2, Standard X-ray Diffraction Powder Patterns (Data for 30 inorganic substances) 45 cents

NBS Circular 539, Volume 3, Standard X-ray Diffraction Powder Patterns (Data for 34 inorganic substances) 45 cents

NBS Circular 539, Volume 4, Standard X-ray Diffraction Powder Patterns (Data for 42 inorganic substances) 45 cents

NBS Circular 539, Volume 5, Standard X-ray Diffraction Powder Patterns (Data for 45 inorganic substances) 45 cents

NBS Circular 539, Volume 6, Standard X-ray Diffraction Powder Patterns (Data for 44 inorganic substances) 40 cents

NBS Circular 539, Volume 7, Standard X-ray Diffraction Powder Patterns (Data for 53 substances) 40 cents

NBS Circular 539, Volume 8, Standard X-ray Diffraction Powder Patterns (Data for 61 substances) 45 cents

NBS Circular 539, Volume 9, Standard X-ray Diffraction Powder Patterns (Data for 43 substances) 40 cents

NBS Circular 539, Volume 10, Standard X-ray Diffraction Powder Patterns (Data for 40 substances) 40 cents

NBS Monograph 25, Section 1, Standard X-ray Diffraction Powder Patterns (Data for 46 substances) 40 cents

NBS Monograph 25, Section 2, Standard X-ray Diffraction Powder Patterns (Data for 37 substances) 35 cents

Send orders with remittance to Superintendent of Documents, U.S. Government Printing Office, Washington, D.C.

20402. Remittances from foreign countries should include an additional one-fourth of the purchase price for postage.

Those wishing to be notified of future issues should send mailing address to the Government Printing Office. 


\title{
STANDARD X-RAY DIFFRACTION POWDER PATTERNS
}

\section{Section 3-Data for 51 Substances}

\author{
Howard E. Swanson, Marlene Cook Morris, ${ }^{1}$ \\ Eloise H. Evans, ${ }^{1}$ and Linda Ulmer
}

\begin{abstract}
Standard x-ray diffraction powder patterns are presented for the following fifty-one substances: $3 \mathrm{Al}_{2} \mathrm{O}_{3} \cdot 2 \mathrm{SiO}_{2}$, (mullite); $\left(\mathrm{NH}_{4}\right)_{2} \mathrm{BeF}_{4} ; \mathrm{NH}_{4} \mathrm{BF}_{4} ; \mathrm{Sb}_{2} \mathrm{Se}_{3} * ; \mathrm{Sb}_{2} \mathrm{Te}_{3} * ; \mathrm{As}_{2} \mathrm{O}_{3} *$, claudetite; $\mathrm{BaBr}_{2} \cdot \mathrm{H}_{2} \mathrm{O}^{*} ; \mathrm{BaSnO}_{3} ; \mathrm{BiPO}_{4}$ (monoclinic); $\mathrm{BiPO}_{4} *$ (trigonal); $\mathrm{BiVO}_{4}$ * (tetragonal); $\mathrm{BiVO}_{4} *$ (monoclinic); $\mathrm{Bi}_{2} \mathrm{Te}_{3}$, (tellurobismuthite); $\mathrm{Bi}_{2} \mathrm{O}_{3}$, (bismite); $\mathrm{Cd}\left(\mathrm{ClO}_{4}\right)_{2} \cdot 6 \mathrm{H}_{2} \mathrm{O} *$; $\mathrm{CdSO}_{4} ; \mathrm{CdTe} ; \mathrm{Ca}_{5} \mathrm{~F}\left(\mathrm{PO}_{4}\right)_{3}$, (fluorapatite) ; $\mathrm{CeNbTiO}_{6}{ }^{*}$, (eschynite); $\mathrm{Cs}_{2} \mathrm{CrO}_{4} *$; $\mathrm{CsF} ; \mathrm{CoSiF}_{6} \cdot-$ $6 \mathrm{H}_{2} \mathrm{O}^{*} ; \mathrm{Co}\left(\mathrm{ClO}_{4}\right)_{2} \cdot 6 \mathrm{H}_{2} \mathrm{O}^{*}$; $\mathrm{CuSO}_{4}$, (chalcocyanite); $\mathrm{DyAsO}_{4}^{*} ; \mathrm{ErAsO}_{4} *$; EuAsO ${ }_{4}^{*} ; \mathrm{GaAs}^{*}$; $\mathrm{HoAsO}_{4}{ }^{*}$; InAs; $\mathrm{LaAsO}_{4}{ }^{*}$; $\mathrm{LaNbTiO}_{6}{ }^{*} ; \mathrm{Li}_{3} \mathrm{PO}_{4}$, (lithiophosphate) low form; $\mathrm{Li}_{3} \mathrm{PO}_{4}$ high form: $\mathrm{MgNH}_{4} \mathrm{PO}_{4} \cdot 6 \mathrm{H}_{2} \mathrm{O}$, (struvite); $\mathrm{KClO}_{3} ; \mathrm{KLiSO}_{4}^{*} ; \mathrm{K}_{3} \mathrm{CrO}_{8} * ; \mathrm{K}_{2} \mathrm{Zn}_{2} \mathrm{~V}_{10} \mathrm{O}_{28} \cdot 16 \mathrm{H}_{2} \mathrm{O}^{*} ; \mathrm{Rb}_{2-}$ $\mathrm{CrO}_{4}{ }^{*} ; \mathrm{AgSbTe}_{2} * ; \mathrm{NaMg}_{3} \mathrm{Al}_{6} \mathrm{~B}_{3} \mathrm{Si}_{6} \mathrm{O}_{27}(\mathrm{OH})_{4}^{*}$, dravite; $\mathrm{Na}_{3} \mathrm{P}_{3} \mathrm{O}_{9} ; \mathrm{Na}_{3} \mathrm{P}_{3} \mathrm{O}_{9} \cdot \mathrm{H}_{2} \mathrm{O} ; \mathrm{SnF}{ }_{2} * ; \mathrm{SrO}_{2} \mathrm{~B}_{2} \mathrm{O}_{3} *$; $\mathrm{TbAsO}_{4}{ }^{*} ; \mathrm{TlCrO}_{4}{ }^{*} ; \mathrm{TmAsO}_{4} * ; \mathrm{TiO}_{2}$, brookite; and $\mathrm{ZnTe}$. Twenty-one are to replace patterns already given in the X-ray Powder Data File issued by the American Society for Testing and Materials, and thirty patterns indicated by asterisks are for substances not previously included. The patterns were made with a Geiger counter x-ray diffractometer, using samples of high purity. When possible, the $d$-values were assigned Miller indices determined by comparison with calculated interplanar spacings and from space group extinctions. The densities and lattice constants were calculated, and the refractive indices were measured whenever possible.
\end{abstract}

\section{INTRODUCTION}

The X-ray Powder Data File [1] ${ }^{2}$ is a compilation of diffraction patterns from many sources and is used for the identification of unknown crystalline materials by matching spacing and intensity measurements. The National Bureau of Standards in its program $^{3}$ for the revision and evaluation of published x-ray data for the X-ray Powder Data File presents data in this report for 51 compounds. This compilation is the thirteenth of a series of "Standard X-ray Diffraction Patterns." 4 The designation "Circular 539 " used for the first 10 volumes has been discontinued in favor of the new series, "Monograph 25." This compilation is the third section of the new series Monograph 25. Included are patterns recommended to replace data on 26 cards now present in the File. The other patterns are for 30 compounds not included in the File. These compounds are: antimony selenide; antimony telluride; arsenic oxide, claudetite; barium bromide monohydrate; bismuth orthophosphate (trigonal); bismuth orthovanadate (tetragonal); bismuth orthovanadate (monoclinic); cadium perchlorate hexahydrate; cerium niobium titanium oxide, eschynite; cesium chromate; cobalt fluosilicate

1 Research Associate at the National Bureau of Standards sponsored by the Joint Committee on Chemical Analysis by Powder Diffraction Methods. 2 Figures in brackets indicate the literature refercnces at the end of each section of this paper.

3 This project is sponsored by the Joint Committee on Chemical Analysis by Powder Diffraction Methods. This committee is composed of members from the American Society for Testing and Materials, the American Crystallographic Association, and the British Institute of Fhssics. Financial support is also provided by the National Bureau of Standards.

4 Other rolumes were published as follows: Circular 539 Vol. 1 and Vol. 2. June 1953; Vol. 3, June 1054; Vol. 4, March 1955; Vol. 5, October 1955; Vol. 6, September 1956; Vol. 7, September 1957; Vol. 8, A pril 1959; Vol. 9, February 1960; Vol. 10, September 1960; Monograph 25 Section 1, March 1962; and Section 2, May 1963. hexahydrate; cobalt perchlorate hexahydrate; dysprosium arsenate; erbium arsenate; europium arsenate; gallium arsenide; holmium arsenate; lanthanum arsenate; lanthanum niobium titanium oxide; potassium lithium sulfate; potassium perchromate; potassium zinc decavanadate 16 -hydrate; rubidium chromate; silver antimony telluride; sodium magnesium aluminum boron hydroxy silicate, dravite; stannous fluoride; strontium 1:1 borate; terbium arsenate; thallium chromate; and thulium arsenate.

The experimental procedure and general plan of this Monograph section 3 have not changed greatly from previous publications. However, the basic technique is discussed, in this section, with minor changes.

Powder data cards. Under this heading are given the Powder Data File card numbers, the three strongest lines, and the literature references for each card. Cards listed through the 1962 index to the Powder Data File are included in the table.

Additional published patterns. Literature references for patterns that have not been published as Powder Data cards are listed.

NBS sample. Many of the samples used to make NBS patterns were special preparations of high purity obtained from a variety of sources or prepared in small quantities in our laboratory by J. deGroot. Unless otherwise noted, the spectrographic analyses were done at NBS after preparation of the sample was completed. The limit of detection for the alkali elements was 0.05 percent for the spectrographic analysis. A microscopic inspection for phase purity was made on the nonopaque materials during the refractive index determination. Another check of phase purity was 
usually provided by the x-ray pattern itself, when it was indexed by comparison with theoretical $d$-values. Treating the sample by appropriate annealing, recrystallization, or heating in hydrothermal bombs improved the quality of most of the patterns. The refractive index measurements were made by grain-immersion methods in white light, using oils standardized in sodium light, and covering the range 1.40 to 2.00 .

X-ray techniques. At least three patterns for intensity measurements were prepared to check reproducibility. Samples that gave satisfactory intensity patterns usually had an average particlesize smaller than $10 \mu$. [2]. In order to avoid the orientation effects when samples are packed or pressed, a sample holder was made that had an extended rectangular cavity open at its top face and end. To prepare the sample, a glass slide was clamped over the top face to form a temporary cavity wall. (See fig. 1.) The powdered sample was then drifted into the remaining end opening while the holder was held in a vertical position. With the sample holder returned to a horizontal position, the glass slide was carefully removed so that the sample surface could be exposed to the $\mathrm{x}$-ray beam (as shown in fig. 2). To powders that did not flow readily or were prone to orient excessively, approximately 50-volume percent of finely-ground silica-gel was added as a diluent. The intensities of the diffraction lines were measured as peak heights above background and were expressed in percentages of the intensity of the strongest line. Additional patterns were obtained for $d$-value measurements. Specimens for these patterns were prepared by packing into a shallow holder a sample containing approximately 5-wt percent tungsten powder that served as an internal standard. When tungsten lines were found to interfere, 25 percent silver was used in place of tungsten. If the internal standard correction varied along the length of the pattern, linear interpolations were used for the regions between the peaks of the standard. For low values of $2 \theta$, the pattern peak was measured in the center, at a place averaging about 75 percent of the peak height. For higher values of $2 \theta$, where the $\alpha_{1}$ and $\alpha_{2}$ peaks were separated, the $\alpha_{1}$ peak was measured in the same way. The internal standard correction appropriate to each region was then applied to the measurement of $2 \theta$. The internal standard lattice constants used were $3.1648 \AA$ for tungsten and $4.0861 \AA$ for silver at $25^{\circ} \mathrm{C}$. as determined by Jette and Foote [3]. All of the NBS patterns, unless otherwise noted, are made at $25^{\circ} \mathrm{C}$, using either filtered copper or cobalt radiation $\left(K \alpha_{1}\right)$, having the wavelengths $1.5405 \AA$, and $1.7889 \AA$, respectively.

Structural data. For cubic materials a value for the lattice constant was calculated for each $d$-value. However, the constant reported is that obtained by averaging the last five lines because of the greater accuracy of measurement in the large-angle region of the pattern. The unit cell values for each noncubic substance were determined by means of a least-squares calculation made on the IBM 7090 , using those $d$-values for which only one set of Miller indices could be assigned. The number of significant figures reported for $d$-values in the NBS pattern is limited by the quality of each sample as indicated by residuals obtained from least squares refinement. A portion of the indexing and cell refinement calculation was performed on a Burroughs B 220 computer at the United States Geological Survey using a program developed by H. T. Evans, Jr.

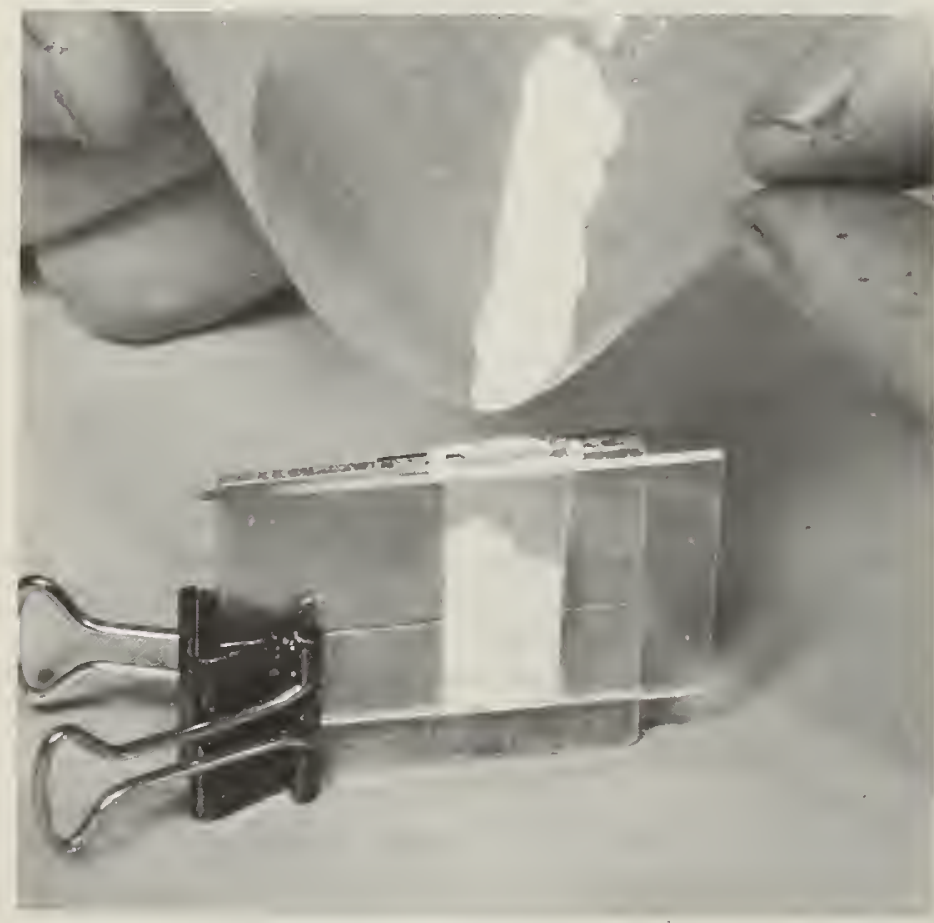

FIGURE 1

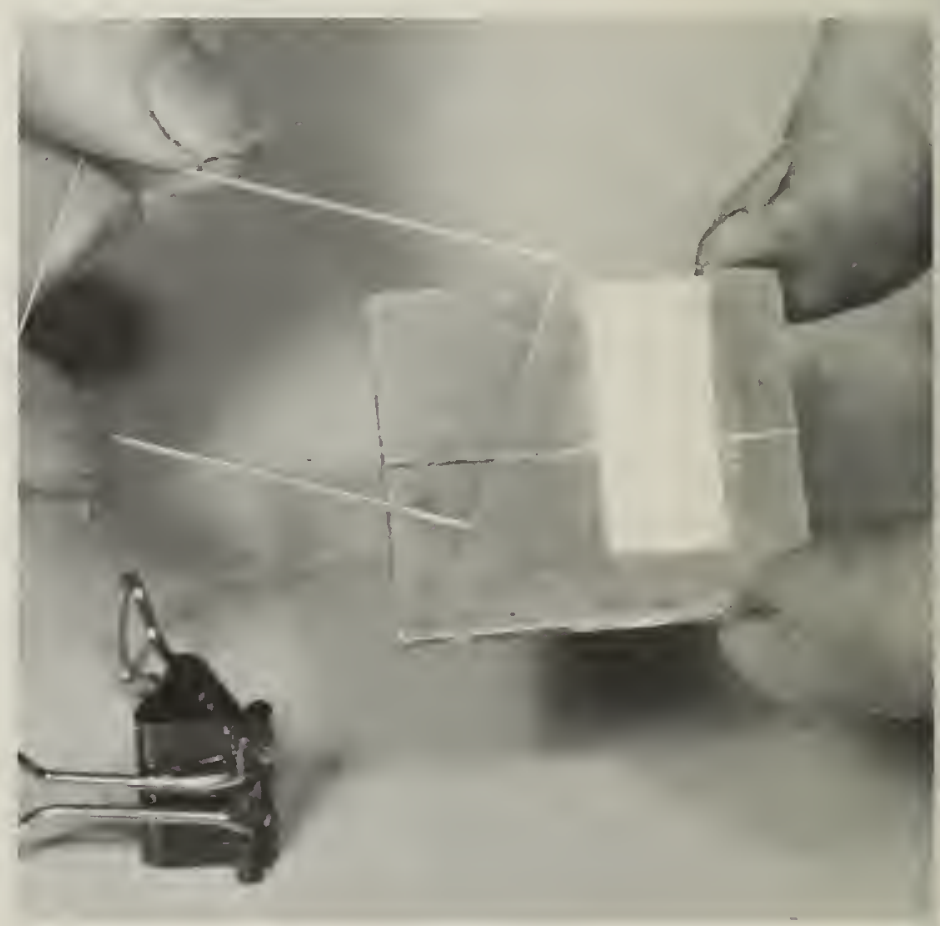

FigURe 2 
D. E. Appleman, and D. Handwerker. Lattice constant errors are given only for data refined on that program and are based on least squares refinement of the variance-covariance matrix derived from the unweighted $\triangle \theta$ residuals.

Published unit cell data in $\mathrm{kX}$ units were converted to angstrom units using the factor 1.00202 as recommended by an international conference of crystallographers [4].

The space groups are listed with both the Schöenflies and short Hermann-Mauguin symbols as well as the space group numbers given in the International Tables for X-ray Crystallography [5].

Orthorhombic cell dimensions are presented according to the Dana convention [6] $b>a>c$.

The densities calculated from the NBS lattice constants are expressed in grams per cubic centimeter and are computed with atomic weights based on carbon 12 [7], and the Avogadro number $\left(6.02252 \times 10^{23}\right)$.

\section{References}

[1] Index to the X-ray Powder Data File, American Society for Testing and Materials, 1916 Race Street, Philadelphia 3, Pa. (1962).

[2] L. Alexander, H. P. Klug, and E. Kummer, Statistical factors affecting the intensity of x-rays diffracted by crystalline powders, J. Appl. Phys. 19, No. 8, 742-753 (1948).

[3] E. R. Jette and F. Foote, Precision determination of lattice constants, J. Chem. Phys. 3, 605-616 (1935).

[4] The conversion factor for $\mathrm{kX}$ units to angstrom units, J. Sci. Inst. 24, 27 (1947).

[5] International Tables for X-ray Crystallography, 1, 1952.

[6] Dana's System of Mineralogy, 1, 6 (1944).

[7] International Union of Pure and Applied Chemistry, Chem. Eng. News, Nov. 20, 43 (1961).

\section{Aluminum 3:2 Silicate (mullite) ${ }^{*} 3 \mathrm{Al}_{2} \mathrm{O}_{3} \cdot 2 \mathrm{SiO}_{2}$ (orthorhombic)}

\section{Powder data cards}

\begin{tabular}{|c|c|c|}
\hline $\begin{array}{c}\text { Card } \\
\text { number }\end{array}$ & $\begin{array}{l}\text { Index } \\
\text { lines }\end{array}$ & Source \\
\hline $6-0258$ & $\begin{array}{l}\text { 3. } 38 \\
\text { 2. } 20\end{array}$ & \\
\hline 10-394 & $\begin{array}{l}\text { 3. } 41 \\
\text { 3. } 39 \\
\text { 3. } 42 \\
\text { 2. } 54\end{array}$ & H. Scholze [2] 1955. \\
\hline
\end{tabular}

Additional published patterns. Norton [3] 1925, Navias and Davey [4] 1925; Wyckoff, Grieg, and Bowen [5] 1926; Mark and Rosbaud [6] 1926; Nahmias [7] 1933; Comeforo, Fischer, and Bradley [8] 1948; and Kurylenko [9] 1952.

NBS sample. The sample of aluminum $3: 2$ silicate was prepared at NBS by C. Robbins. Gamma $\mathrm{Al}_{2} \mathrm{O}_{3}$ and $\mathrm{SiO}_{2} \cdot n \mathrm{H}_{2} \mathrm{O}$ were mechanically mixed in stoichiometric proportions of $3 \mathrm{Al}_{2} \mathrm{O}_{3}$ to $2 \mathrm{SiO}_{2} \cdot n \mathrm{H}_{2} \mathrm{O}$. This mixture was pressed into pellets and fired at $1400{ }^{\circ} \mathrm{C}$ and $1500{ }^{\circ} \mathrm{C}$ with little reaction, then remixed and fired in an open system at $1700{ }^{\circ} \mathrm{C}$ for $24 \mathrm{hr}$. Reaction was nearly complete. It was crushed, reground in an agate mortar and refired at $1725^{\circ} \mathrm{C}$ for $24 \mathrm{hr}$. Chemical analysis of the finished product showed 61.6 mole percent $\mathrm{Al}_{2} \mathrm{O}_{3}$ and 38.4 mole percent $\mathrm{SiO}_{2}$ instead of the beginning percentages of 60 and 40 in the $3: 2$ mixture. Spectrographic analysis showed the following major impurities: 0.01 to 0.1 percent iron, and 0.001 to 0.01 percent each of calcium, chromium, magnesium, manganese, nickel, titanium, and zirconium.

* Some disagreement exists in the literature as to which ratio or ratios of alumina to silica should be considered for mullite $(3: 2,2: 1$, or both).

This sample was prepared as $3: 2$ at the request of P. Schroth of the Armco Steel Corp. to be used as an identification, diffraction standard for mullite in refractory brick.
The sample was colorless and optically positive with the indices of refraction $\mathrm{N}_{\alpha}=1.637, \mathrm{~N}_{\beta}=$ 1.641 , and $\mathrm{N}_{\gamma}=1.652$.

The $d$-values of the three strongest lines are: $3.390,3.428$ and $2.206 \AA$.

Structural data. Sadanaga, Tokonami, and Takéuchi [10] in 1962 determined that mullite has the space group $\mathrm{D}_{2 \mathrm{~h}}^{9}-\mathrm{Pbam}$ (No. 55). Wyckoff, Grieg, and Bowen [5] in 1926 determined that mullite has $3 / 4\left(3 \mathrm{Al}_{2} \mathrm{O}_{3} \cdot 2 \mathrm{SiO}_{2}\right)$ per unit cell. Several lattice constants have been converted from $\mathrm{kX}$ to angstrom units for comparison with the NBS values.

\section{Lattice constants}

\begin{tabular}{|c|c|c|c|c|}
\hline & & $a$ & $b$ & $c$ \\
\hline & & $A$ & $\AA$ & $\AA$ \\
\hline 1926 & Wyckoff, Grieg, & 7. 52 & 7. 68 & 2.86 \\
\hline 1928 & Taylor $[11]_{-}$ & 7. 51 & 7. 65 & 2. 88 \\
\hline 1933 & Nahmias [7] & 7. 54 & 7. 67 & 2. 90 \\
\hline 1952 & Kúrylenko $[9]_{-}$ & 7. 580 & 7. 689 & 2. 895 \\
\hline 1955 & Scholze $[2] \ldots$ & 7. 537 & 7. 671 & 2. 878 \\
\hline 1960 & $\begin{array}{l}\text { Agrell and Smith } \\
\text { [12]. }\end{array}$ & 7. 5582 & 7. 6878 & 2. 8843 \\
\hline 1962 & $\begin{array}{l}\text { Sadanaga, } \\
\text { Tokonami, } \\
\text { and Takéuchi } \\
\text { [10]. }\end{array}$ & $* 7.583$ & *7. 681 & *2. 8854 \\
\hline 1963 & $\begin{array}{l}\text { National Bureau } \\
\text { of Standards } \\
\text { at } 25^{\circ} \mathrm{C} \text {. }\end{array}$ & $\begin{array}{r}7.5456 \\
\pm .0004\end{array}$ & $\begin{array}{r}7.6898 \\
\pm .0005\end{array}$ & $\begin{array}{r}2.8842 \\
\pm .0002\end{array}$ \\
\hline
\end{tabular}

*These values are for $2 \mathrm{Al}_{2} \mathrm{O}_{3} \cdot \mathrm{SiO}_{3}$.

The density calculated from the NBS lattice constants is $3.170 \mathrm{~g} / \mathrm{cm}^{3}$ at $25^{\circ} \mathrm{C}$. 


\section{Aluminum 3:2 Silicate (mullite) $3 \mathrm{Al}_{2} \mathrm{O}_{2} \cdot 2 \mathrm{SiO}_{2}$ (orthorhombic)-Continued}

\begin{tabular}{|c|c|c|c|c|c|}
\hline \multirow[t]{2}{*}{$h k l$} & \multicolumn{2}{|c|}{$\begin{array}{l}\text { Internal Standard, } \\
\text { Tungsten, } a=3.1648 \AA \\
\mathrm{Cu}, 1.5405 \AA \text { at } 25^{\circ} \mathrm{C}\end{array}$} & \multirow[t]{2}{*}{$h k l$} & \multicolumn{2}{|c|}{$\begin{array}{l}\text { Internal Stannard, } \\
\text { Tungsten, } a=3.1648 \AA \\
\mathrm{Cu}, 1.5405 \AA \text { at } 25^{\circ} \mathrm{C}\end{array}$} \\
\hline & $d$ & $I$ & & $d$ & $I$ \\
\hline $\begin{array}{l}110 \\
200 \\
120 \\
210 \\
001\end{array}$ & 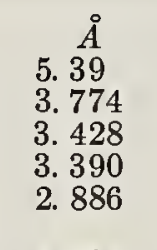 & $\begin{array}{r}48 \\
8 \\
96 \\
100 \\
21\end{array}$ & $\begin{array}{l}112 \\
341 \\
440 \\
151 \\
122 \\
212\end{array}$ & $\begin{array}{c}\AA \\
\text { 1. } 3932 \\
1.3494 \\
\text { 1. } 3462 \\
\text { 1. } 3356 \\
\text { 1. } 3290 \\
\text { 1. } 3266\end{array}$ & $\begin{array}{r}<2 \\
6 \\
12 \\
5\end{array}$ \\
\hline $\begin{array}{l}220 \\
111 \\
130 \\
310 \\
021\end{array}$ & $\begin{array}{l}\text { 2. } 694 \\
\text { 2. } 542 \\
\text { 2. } 428 \\
\text { 2. } 393 \\
\text { 2. } 308\end{array}$ & $\begin{array}{r}40 \\
52 \\
13 \\
<2 \\
3\end{array}$ & $\begin{array}{l}511 \\
350 \\
530 \\
060 \\
251\end{array}$ & $\begin{array}{l}\text { 1. } 3172 \\
\text { 1. } 3120 \\
\text { 1. } 3004 \\
\text { 1. } 2814 \\
1 \text {. } 2771\end{array}$ & $\begin{array}{r}4 \\
3 \\
4 \\
7 \\
13\end{array}$ \\
\hline $\begin{array}{l}201 \\
121 \\
230 \\
320 \\
221\end{array}$ & $\begin{array}{l}\text { 2. } 292 \\
\text { 2. } 206 \\
2.121 \\
\text { 2. } 106 \\
\text { 1. } 969\end{array}$ & $\begin{array}{r}19 \\
61 \\
23 \\
7 \\
2\end{array}$ & $\begin{array}{l}222 \\
521 \\
600 \\
132 \\
312\end{array}$ & $\begin{array}{l}\text { 1. } 2714 \\
\text { 1. } 2630 \\
\text { 1. } 2574 \\
\text { 1. } 2396 \\
1.2349\end{array}$ & $\begin{array}{r}6 \\
12 \\
<2 \\
6 \\
2\end{array}$ \\
\hline $\begin{array}{l}040 \\
400 \\
140 \\
311 \\
330\end{array}$ & $\begin{array}{l}\text { 1. } 923 \\
\text { 1. } 887 \\
1.863 \\
1.841 \\
1.7954\end{array}$ & $\begin{array}{r}2 \\
8 \\
<2 \\
10 \\
<2\end{array}$ & $\begin{array}{l}441 \\
260 \\
232 \\
531 \\
402\end{array}$ & $\begin{array}{l}\text { 1. } 2199 \\
\text { 1. } 2131 \\
\text { 1. } 1924 \\
\text { 1. } 1855 \\
\text { 1. } 1457\end{array}$ & $\begin{array}{r}2 \\
<2 \\
4 \\
3 \\
<2\end{array}$ \\
\hline $\begin{array}{l}240 \\
321 \\
420 \\
041 \\
401\end{array}$ & $\begin{array}{l}1.7125 \\
1.7001 \\
1.6940 \\
1.5999 \\
1.5786\end{array}$ & $\begin{array}{r}6 \\
14 \\
10 \\
20 \\
11\end{array}$ & $\begin{array}{l}261 \\
242 \\
422 \\
270 \\
171\end{array}$ & $\begin{array}{l}\text { 1. } 1190 \\
\text { 1. } 1032 \\
\text { 1. } 0981 \\
\text { 1. } 0548 \\
1.0172\end{array}$ & $\begin{array}{r}1 \\
4 \\
5 \\
<2 \\
4\end{array}$ \\
\hline $\begin{array}{l}141 \\
411 \\
331 \\
150 \\
510\end{array}$ & $\begin{array}{l}\text { 1. } 5644 \\
\text { 1. } 5461 \\
\text { 1. } 5242 \\
\text { 1. } 5067 \\
1.4811\end{array}$ & $\begin{array}{r}2 \\
2 \\
37 \\
<2 \\
<2\end{array}$ & $\begin{array}{c}252 \\
370,522\end{array}$ & $\begin{array}{l}\text { 1. } 0133 \\
\text { 1. } 0065\end{array}$ & $\begin{array}{l}4 \\
8\end{array}$ \\
\hline $\begin{array}{l}241 \\
421 \\
002 \\
250 \\
520\end{array}$ & $\begin{array}{l}\text { 1. } 4731 \\
\text { 1. } 4605 \\
\text { 1. } 4421 \\
\text { 1. } 4240 \\
\text { 1. } 4046\end{array}$ & $\begin{array}{r}<2 \\
7 \\
17 \\
3 \\
7\end{array}$ & & & \\
\hline
\end{tabular}

\section{References}

[1] F. H. Gillery, The Pennsylvania State Univ., University Park, $\mathrm{Pa}$

[2] H. Scholze, Zum Sillimanit-Mullit Problem, Ber. deut. keram. Ges. 32, 381-5 (1955).

[3] J. T. Norton, An x-ray study of natural and artificial sillimanite, J. Am. Ceram. Soc. 8, 401 (1925).

[4] L. Navias and W. P. Davey, Differentiation between mullite and sillimanite by their x-ray diffraction patterns, J. Am. Ceram. Soc. 8, 640 (1925).

[5] R. W. G. Wyckoff, J. W. Grieg, and N. L. Bowen, The x-ray diffraction patterns of mullite and sillimanite, Âm. J. Sci. 11, 459-72 (1926).

[6] H. Mark und P. Rosbaud, Úber die Struktur der Aluminiumsilikate vom Typus $\mathrm{Al}_{2} \mathrm{SiO}_{5}$ und des Pseudobrookits, Neues Jahr. Mineral. Geol. Beilage B. 54A, 127-64 (1926).
[7] M. E. Nahmias, Bauxites et mullites, etudiées au moyen des rayons X. Z. Krist. 85, 355-69 (1933).

[8] J. E. Comeforo, R. B. Fischer, and W. F. Bradley, Mullitization of kaolinite, J. Am. Ceram. Soc. 31, 254-9 (1948).

[9] C. Kurylenko, Ensayo sobre la estructura de la mullita, pR Revista cientifica 2, 107-120 (1952).

[10] R. Sadanaga, M. Tokonami, and Y. Takéuchi, The structure of mullite, $2 \mathrm{Al}_{2} \mathrm{O}_{3} \cdot \mathrm{SiO}_{2}$ and relationship with the structures of sillimanite and andalusite, Acta Cryst. 15, 65 (1962).

[11] W. H. Taylor, The structure of sillimanite and mullite, Z. Krist. 68, 503-521 (1928).

[12] S. O. Agrell and J. V. Smith, Cell dimensions, solid solutions, polymorphism, and identification of mullite and sillimanite, J. Am. Ceram. Soc. 43, 69 (1960). 
Ammonium Fluoberyllate, $\left(\mathrm{NH}_{4}\right)_{2} \mathrm{BeF}_{4}$ (orthorhombic)

\section{Powder data cards}

\begin{tabular}{|c|c|c|}
\hline $\begin{array}{c}\text { Card } \\
\text { number }\end{array}$ & $\begin{array}{c}\text { Index } \\
\text { lines }\end{array}$ & Source \\
\hline $3-0885$ & $\begin{array}{l}2.48 \\
2.27 \\
4.31\end{array}$ & The Dow Chemical Co. \\
\hline
\end{tabular}

Additional published patterns. None.

NBS sample. The sample of ammonium fluoberyllate was obtained from $\mathrm{K}$ and $\mathrm{K}$ Laboratories, Jamaica, Long Island, N.Y. Spectrographic analysis showed the following major impurities: 0.001 to 0.01 percent each of aluminum, calcium, and silicon.

The sample was colorless, and optically nega tive with $\mathrm{N}_{\alpha}=1.397, \mathrm{~N}_{\beta}=1.401$, and $\mathrm{N}_{\gamma}=1.403$.

The $d$-values of the three strongest lines are: $4.275,2.478$, and $4.312 \AA$.

Structural data. Hultgren [1] in 1934 determined that ammonium fluoberyllate belongs to the space group $\mathrm{D}_{2 \mathrm{~h}}^{16}-\mathrm{Pnam}$ (No. 62 ) with $4\left[\left(\mathrm{NH}_{4}\right)_{2}\right.$ $\mathrm{BeF}_{4}$ ] per unit cell. The lattice constants of Mukherjee [2] have been converted from $\mathrm{kX}$ to angstrom units for comparison with the NBS values.

\section{Lattice constants}

\begin{tabular}{|c|c|c|c|c|}
\hline & & $a$ & $b$ & $c$ \\
\hline $\begin{array}{l}1934 \\
1945 \\
1963\end{array}$ & $\begin{array}{l}\text { Hultgren }[1] \\
\text { Mukherjee }[2] \\
\text { National Bureau } \\
\text { of Standards at } \\
25{ }^{\circ} \mathrm{C} \text {. }\end{array}$ & $\begin{array}{l}\breve{A} \\
7.5 \\
7.51 \\
7.645 \\
\pm .001\end{array}$ & $\begin{array}{l}\stackrel{\AA}{1} \\
10.2 \\
10.41 \\
10.450 \\
\pm .001\end{array}$ & $\begin{array}{l}\quad \AA \\
\text { 5. } \\
5.90 \\
5.929 \\
\pm .001\end{array}$ \\
\hline
\end{tabular}

The density of ammonium fluoberyllate calculated from NBS lattice constants in $1.698 \mathrm{~g} / \mathrm{cm}^{3}$ at $25{ }^{\circ} \mathrm{C}$.

\begin{tabular}{|c|c|c|}
\hline \multirow[t]{2}{*}{$h k l l$} & \multicolumn{2}{|c|}{$\begin{array}{l}\text { Internal Standard, } \\
\text { Tungsten, } a=3.1648 \AA \\
\mathrm{Cu}, 1.5405 \AA \text { at } 25^{\circ} \mathrm{C}\end{array}$} \\
\hline & $d$ & $I$ \\
\hline $\begin{array}{l}020 \\
011 \\
120 \\
111 \\
200\end{array}$ & $\begin{array}{cc} & \AA \\
\text { 5. } 229 \\
\text { 5. } 157 \\
\text { 4. } 312 \\
\text { 4. } 275 \\
\text { 3. } 821\end{array}$ & $\begin{array}{r}5 \\
15 \\
90 \\
100 \\
36\end{array}$ \\
\hline $\begin{array}{l}201 \\
220 \\
031 \\
002 \\
131\end{array}$ & $\begin{array}{l}\text { 3. } 212 \\
\text { 3. } 085 \\
\text { 3. } 003 \\
\text { 2. } 962 \\
\text { 2. } 794\end{array}$ & $\begin{array}{l}<2 \\
38 \\
46 \\
40 \\
<2\end{array}$ \\
\hline $\begin{array}{l}112 \\
040 \\
230 \\
310 \\
122\end{array}$ & $\begin{array}{l}\text { 2. } 671 \\
2.612 \\
2.575 \\
2.478 \\
2.443\end{array}$ & $\begin{array}{l}23 \\
48 \\
15 \\
96 \\
69\end{array}$ \\
\hline $\begin{array}{l}231 \\
202 \\
320 \\
311 \\
132\end{array}$ & $\begin{array}{l}\text { 2. } 362 \\
\text { 2. } 342 \\
\text { 2. } 289 \\
\text { 2. } 284 \\
\text { 2. } 164\end{array}$ & $\begin{array}{r}15 \\
<2 \\
75 \\
<2 \\
32\end{array}$ \\
\hline $\begin{array}{c}321,222 \\
330 \\
241 \\
051 \\
013,331\end{array}$ & $\begin{array}{l}\text { 2. } 136 \\
\text { 2. } 056 \\
\text { 2. } 027 \\
\text { 1. } 970 \\
\text { 1. } 942\end{array}$ & $\begin{array}{r}25 \\
16 \\
3 \\
3 \\
26\end{array}$ \\
\hline $\begin{array}{l}400 \\
410 \\
250 \\
401 \\
322\end{array}$ & $\begin{array}{l}\text { 1. } 911 \\
\text { 1. } 879 \\
\text { 1. } 8339 \\
\text { 1. } 8192 \\
\text { 1. } 8124\end{array}$ & $\begin{array}{r}4 \\
<2 \\
10 \\
2 \\
<2\end{array}$ \\
\hline $\begin{array}{c}411,420 \\
251 \\
341 \\
033 \\
160\end{array}$ & $\begin{array}{l}\text { 1. } 7934 \\
\text { 1. } 7526 \\
\text { 1. } 7552 \\
\text { 1. } 7193 \\
\text { 1. } 6977\end{array}$ & $\begin{array}{r}2 \\
7 \\
<2 \\
3 \\
4\end{array}$ \\
\hline $\begin{array}{c}133 \\
350 \\
402 \\
233 \\
252,351\end{array}$ & $\begin{array}{l}\text { 1. } 6765 \\
\text { 1. } 6168 \\
\text { 1. } 6070 \\
\text { 1. } 5668 \\
\text { 1. } 5598\end{array}$ & $\begin{array}{r}4 \\
<2 \\
5 \\
<2 \\
2\end{array}$ \\
\hline $\begin{array}{l}510 \\
323 \\
004\end{array}$ & $\begin{array}{l}\text { 1. } 5124 \\
\text { 1. } 4963 \\
\text { 1. } 4821\end{array}$ & $\begin{array}{r}3 \\
<2 \\
6\end{array}$ \\
\hline
\end{tabular}

\section{References}

[1] R. Hultgren, Crystal structures of ammonium beryllium fluoride, Z. Krist. (A) 88, 233-237 (1934).

[2] P. L. Mukherjee, Crystal structures of metallic fluoberyllates, double fluoberyllates and sulphatofluoberyllates, Indian J. of Phys. 18, 148 (1945). 
Powder data cards.

\begin{tabular}{|c|c|c|}
\hline $\begin{array}{c}\text { Card } \\
\text { number }\end{array}$ & $\begin{array}{c}\text { Index } \\
\text { lines }\end{array}$ & Source \\
\hline $1-0335$ & $\begin{array}{r}4.50 \\
3.55 \\
3.18\end{array}$ & Hanawalt, Rinn, and Frevel \\
& [2] 1938. \\
\hline
\end{tabular}

Additional published patterns. None.

NBS sample. The sample of ammonium fluoborate was obtained from $\mathrm{K}$ and $\mathrm{K}$ Laboratories, Jamaica, Long Island, N.Y. Spectrographic analysis showed the following major impurities: 0.1 to 1.0 percent each of calcium and phosphorus and 0.001 to 0.01 percent each of iron, titanium, and strontium.

The sample was colorless and had extremely low birefringence and indices of refraction.
The $d$-values of the three strongest lines are: $4.482,3.542$, and $3.186 \AA$.

Structural data. Hoard and Blair [1] in 1935 determined that ammonium fluoborate has the space group $\mathrm{D}_{2 \mathrm{~h}}^{16}-\mathrm{Pbnm}(\mathrm{No} .62)$ and $4\left(\mathrm{NH}_{4} \mathrm{BF}_{4}\right)$ per unit cell. The cell constants of Hoard and Blair have been converted from $\mathrm{kX}$ to angstrom units for comparison with the NBS values.

\section{Lattice constants}

\begin{tabular}{|c|c|c|c|c|}
\hline & & $a$ & $b$ & $c$ \\
\hline 1935 & $\begin{array}{l}\text { Hoard and Blair } \\
\text { [1]. }\end{array}$ & 7. $\stackrel{\stackrel{\circ}{2}}{24}$ & $\begin{array}{c}\stackrel{\circ}{A} \\
9.08\end{array}$ & $\stackrel{\AA}{A}$ \\
\hline 1963 & $\begin{array}{l}\text { National Bureau } \\
\text { of Standards } \\
\text { at } 26^{\circ} \mathrm{C} \text {. }\end{array}$ & $\begin{array}{r}\text { 7. } 272 \\
\pm .001\end{array}$ & $\begin{array}{r}9.063 \\
\pm .001\end{array}$ & $\begin{array}{r}5.686 \\
\pm .001\end{array}$ \\
\hline
\end{tabular}

\begin{tabular}{|c|c|c|c|c|c|}
\hline \multirow[t]{2}{*}{$h k l$} & \multicolumn{2}{|c|}{$\begin{array}{l}\text { Internal Standard, } \\
\text { Tungsten, } a=3.1648 \AA \\
\mathrm{Cu}, 1.5405 \AA \text { at } 26^{\circ} \mathrm{C}\end{array}$} & \multirow[t]{2}{*}{$h k l$} & \multicolumn{2}{|c|}{$\begin{array}{l}\text { Internal Standard, } \\
\text { Tungsten, } a=3.1648 \AA \\
\mathrm{Cu}, 1.5405 \AA \text { at } 26^{\circ} \mathrm{C}\end{array}$} \\
\hline & $d$ & $I$ & & $d$ & $I$ \\
\hline $\begin{array}{l}110 \\
020 \\
101 \\
120 \\
200\end{array}$ & $\begin{array}{c}\AA \\
\text { 5. } 668 \\
\text { 4. } 528 \\
\text { 4. } 482 \\
\text { 3. } 844 \\
\text { 3. } 635\end{array}$ & $\begin{array}{r}30 \\
58 \\
100 \\
51 \\
51\end{array}$ & $\begin{array}{l}312 \\
410 \\
150 \\
023 \\
142\end{array}$ & $\begin{array}{l}\stackrel{\circ}{A} \\
\text { 1. } 808 \\
\text { 1. } 782 \\
\text { 1. } 759 \\
\text { 1. } 749 \\
\text { 1. } 721\end{array}$ & $\begin{array}{r}8 \\
2 \\
<1 \\
<1 \\
5\end{array}$ \\
\hline $\begin{array}{c}021 \\
210 \\
121 \\
211 \\
002,220\end{array}$ & $\begin{array}{l}\text { 3. } 542 \\
\text { 3. } 376 \\
\text { 3. } 186 \\
\text { 2. } 902 \\
\text { 2. } 839\end{array}$ & $\begin{array}{l}85 \\
11 \\
85 \\
61 \\
35\end{array}$ & $\begin{array}{c}322 \\
411,123 \\
151 \\
213 \\
250\end{array}$ & $\begin{array}{l}\text { 1. } 708 \\
1.701 \\
1.680 \\
1.653 \\
1.622\end{array}$ & $\begin{array}{r}2 \\
<1 \\
3 \\
19 \\
4\end{array}$ \\
\hline $\begin{array}{l}130 \\
112 \\
131 \\
022 \\
310\end{array}$ & $\begin{array}{l}2.792 \\
2.541 \\
2.505 \\
2.409 \\
2.341\end{array}$ & $\begin{array}{r}4 \\
45 \\
12 \\
12 \\
23\end{array}$ & $\begin{array}{c}242 \\
223 \\
430 \\
402 \\
060,412\end{array}$ & $\begin{array}{l}1.593 \\
1.575 \\
1.558 \\
1.532 \\
1.510\end{array}$ & $\begin{array}{r}<1 \\
3 \\
2 \\
1 \\
<1\end{array}$ \\
\hline $\begin{array}{l}230 \\
122 \\
040 \\
202 \\
301\end{array}$ & $\begin{array}{l}\text { 2. } 324 \\
\text { 2. } 286 \\
\text { 2. } 266 \\
\text { 2. } 238 \\
\text { 2. } 232\end{array}$ & $\begin{array}{l}37 \\
35 \\
10 \\
18\end{array}$ & $\begin{array}{l}303 \\
233 \\
043 \\
510 \\
161\end{array}$ & $\begin{array}{l}\text { 1. } 4933 \\
1.4691 \\
\text { 1. } 4535 \\
\text { 1. } 4365 \\
1.4316\end{array}$ & $\begin{array}{r}2 \\
<1 \\
5 \\
4 \\
4\end{array}$ \\
\hline $\begin{array}{c}212 \\
311,140 \\
231 \\
320 \\
041\end{array}$ & $\begin{array}{l}2.176 \\
2.165 \\
2.151 \\
2.138 \\
2.104\end{array}$ & $\begin{array}{r}45 \\
11 \\
5 \\
11\end{array}$ & $\begin{array}{l}004 \\
260 \\
520 \\
441 \\
432\end{array}$ & $\begin{array}{l}\text { 1. } 4217 \\
\text { 1. } 3954 \\
\text { 1. } 3848 \\
\text { 1. } 3755 \\
\text { 1. } 3656\end{array}$ & $\begin{array}{l}2 \\
<1 \\
<1 \\
<1 \\
2\end{array}$ \\
\hline $\begin{array}{l}141 \\
222 \\
330 \\
103 \\
241\end{array}$ & $\begin{array}{l}2.022 \\
2.008 \\
1.891 \\
1.834 \\
1.821\end{array}$ & $\begin{array}{l}3 \\
3 \\
2 \\
2 \\
6\end{array}$ & $\begin{array}{l}521 \\
333 \\
062 \\
530 \\
413\end{array}$ & $\begin{array}{l}\text { 1. } 3459 \\
\text { 1. } 3381 \\
\text { 1. } 3344 \\
\text { 1. } 3104 \\
\text { 1. } 2983\end{array}$ & $\begin{array}{r}<1 \\
1 \\
<1 \\
<1 \\
2\end{array}$ \\
\hline
\end{tabular}




\section{Ammonium Fluoborate, $\mathrm{NH}_{4} \mathrm{BF}_{4}$ (orthorhombic) - Continued}

The density of ammonium fluoborate calculated from the NBS lattice constants is $1.858 \mathrm{~g} / \mathrm{cm}^{3}$ at $26{ }^{\circ} \mathrm{C}$.

Powder data cards. None.

Additional published patterns. Dönges [1] 1950. NBS sample. The sample of antimony selenide was obtained from Semitronics, Inc., Winchester, Mass. It was heated in an evacuated tube at $450{ }^{\circ} \mathrm{C}$ overnight to obtain a sharper pattern. Spectrographic analysis showed the following major impurities: 0.01 to 0.1 percent tellurium and

\begin{tabular}{|c|c|c|}
\hline \multirow[t]{2}{*}{$h k l$} & \multicolumn{2}{|c|}{$\begin{array}{l}\text { Internal Standard, } \\
\text { Tungsten, } a \overline{\bar{\AA}} 3.1648 \AA \\
\mathrm{Cu}, 1.5405 \AA \text { at } 25^{\circ} \mathrm{C}\end{array}$} \\
\hline & $d$ & $I$ \\
\hline $\begin{array}{l}110 \\
020 \\
200 \\
120 \\
220\end{array}$ & $\begin{array}{c}\stackrel{\circ}{A} \\
\text { 8. } 28 \\
\text { 5. } 89 \\
\text { 5. } 82 \\
\text { 5. } 25 \\
\text { 4. } 14\end{array}$ & $\begin{array}{r}7 \\
24 \\
5 \\
55 \\
10\end{array}$ \\
\hline $\begin{array}{l}101 \\
130 \\
310 \\
021 \\
231\end{array}$ & $\begin{array}{ll}\text { 3. } & 76 \\
\text { 3. } & 720 \\
\text { 3. } & 682 \\
\text { 3. } 297 \\
\text { 3. } 253\end{array}$ & $\begin{array}{l}11 \\
32 \\
15 \\
11 \\
71\end{array}$ \\
\hline $\begin{array}{l}211 \\
040 \\
400 \\
221 \\
301\end{array}$ & $\begin{array}{l}\text { 3. } 162 \\
\text { 2. } 945 \\
\text { 2. } 910 \\
\text { 2. } 868 \\
\text { 2. } 776\end{array}$ & $\begin{array}{r}73 \\
2 \\
4 \\
100 \\
62\end{array}$ \\
\hline $\begin{array}{l}330 \\
311 \\
240 \\
420 \\
321\end{array}$ & $\begin{array}{l}\text { 2. } 759 \\
\text { 2. } 703 \\
\text { 2. } 629 \\
\text { 2. } 608 \\
\text { 2. } 513\end{array}$ & $\begin{array}{l}10 \\
22 \\
61 \\
22 \\
31\end{array}$ \\
\hline $\begin{array}{l}041 \\
340 \\
430 \\
141 \\
411\end{array}$ & $\begin{array}{l}\text { 2. } 367 \\
\text { 2. } 345 \\
\text { 2. } 337 \\
\text { 2. } 319 \\
\text { 2. } 303\end{array}$ & $\begin{array}{l}23 \\
13 \\
13 \\
35 \\
12\end{array}$ \\
\hline $\begin{array}{l}510 \\
331 \\
250 \\
520 \\
440\end{array}$ & $\begin{array}{l}\text { 2. } 282 \\
\text { 2. } 268 \\
\text { 2. } 184 \\
\text { 2. } 164 \\
\text { 2. } 070\end{array}$ & $\begin{array}{r}8 \\
7 \\
35 \\
19 \\
9\end{array}$ \\
\hline $\begin{array}{l}431 \\
501 \\
530 \\
151 \\
002 \\
060\end{array}$ & $\begin{array}{l}\text { 2. } 015 \\
\text { 2. } 010 \\
\text { 2. } 002 \\
\text { 1. } 998 \\
\text { 1. } 989 \\
\text { 1. } 963\end{array}$ & $\begin{array}{r}35 \\
31 \\
31 \\
23 \\
26 \\
7\end{array}$ \\
\hline
\end{tabular}

References

[1] J. L. Hoard and V. Blair, The crystal structures of rubidium and ammonium fluoborates, J. Am. Chem. Soc. 5\%, 1985-88 (1935).

[2] J. D. Hanawalt, H. W. Rinn, and L. K. Frevel, Chemical analysis by x-ray diffraction, Ind. Eng. Chem. Anal. Ed. 10, 457-512 (1938).

\section{Antimony Selenide, $\mathrm{Sb}_{2} \mathrm{Se}_{3}$ (orthorhombic)}

0.001 to 0.01 percent each of aluminum and silicon.

The sample was a dark gray opaque powder.

The $d$-values of the three strongest lines aro: $2.868,3.162$, and $3.253 \AA$.

Structural data. Dönges [1] in 1950 determined that antimony selenide has the antimony sulfide structure, the space group $\mathrm{D}_{2 \mathrm{~h}}^{16}-\mathrm{Pbnm}$ (No. $62)$, and $4\left(\mathrm{Sb}_{2} \mathrm{Se}_{3}\right)$ per unit cell.

Lattice constants

\begin{tabular}{|c|c|c|c|c|}
\hline \multirow{3}{*}{$\begin{array}{l}1950 \\
1963\end{array}$} & \multirow{3}{*}{$\begin{array}{l}\text { Dönges [1] } \\
\text { National Bureau } \\
\text { of Standards } \\
\text { at } 25^{\circ} \mathrm{C}\end{array}$} & $a$ & $b$ & $c$ \\
\hline & & $\begin{array}{c}\stackrel{\circ}{A} \\
11.58\end{array}$ & 11. $\frac{\AA}{\AA}$ & $\begin{array}{c}\stackrel{\circ}{A} \\
\text { 3. } 98\end{array}$ \\
\hline & & 11. 633 & 11. 780 & 3. 985 \\
\hline
\end{tabular}

\begin{tabular}{|c|c|c|}
\hline \multirow[t]{2}{*}{$h k l$} & \multicolumn{2}{|c|}{$\begin{array}{l}\text { Internal Standard, } \\
\text { Tungsten, } a=3.1648 \AA \\
\mathrm{Cu}, 1.5405 \AA \text { at } 25^{\circ} \mathrm{C}\end{array}$} \\
\hline & $d$ & $I$ \\
\hline $\begin{array}{l}112 \\
160 \\
610 \\
212\end{array}$ & $\begin{array}{c}\AA \\
\text { 1. } 938 \\
1.936 \\
1.913 \\
1.861\end{array}$ & $\begin{array}{l}7 \\
7 \\
7 \\
8\end{array}$ \\
\hline $\begin{array}{l}620 \\
441 \\
540 \\
351 \\
531\end{array}$ & $\begin{array}{l}\text { 1. } 841 \\
\text { 1. } 837 \\
\text { 1. } 826 \\
\text { 1. } 797 \\
1.788\end{array}$ & $\begin{array}{r}3 \\
4 \\
3 \\
8 \\
15\end{array}$ \\
\hline $\begin{array}{l}061 \\
312 \\
322 \\
621 \\
710\end{array}$ & $\begin{array}{l}\text { 1. } 761 \\
\text { 1. } 752 \\
\text { 1. } 698 \\
\text { 1. } 672 \\
1.646\end{array}$ & $\begin{array}{r}47 \\
20 \\
13 \\
5 \\
5\end{array}$ \\
\hline $\begin{array}{l}142 \\
412 \\
640 \\
270 \\
720\end{array}$ & $\begin{array}{l}\text { 1. } 632 \\
\text { 1. } 628 \\
\text { 1. } 619 \\
\text { 1. } 617 \\
\text { 1. } 600\end{array}$ & $\begin{array}{r}<1 \\
<1 \\
6 \\
8 \\
12\end{array}$ \\
\hline $\begin{array}{l}242 \\
422 \\
370 \\
171\end{array}$ & $\begin{array}{l}1.586 \\
1.582 \\
1.544 \\
1.568\end{array}$ & $\begin{array}{r}17 \\
10 \\
8 \\
5\end{array}$ \\
\hline
\end{tabular}




\section{Antimony Selenide, $\mathrm{Sb}_{2} \mathrm{Se}_{3}$ (orthorhombic) - Continued}

The density of antimony selenide calculated from NBS lattice constants is $5.843 \mathrm{~g} / \mathrm{cm}^{3}$ at $25^{\circ} \mathrm{C}$.

\section{Rèference}

[1] E. Dönges, Utber Selenohalogenide des dreiwertigen Antimons und Wismuts und über Antimon (III). selenid, Z. anorg. Chem. 263, 280-291 (1950).

\section{Antimony Telluride, $\mathrm{Sb}_{2} \mathrm{Te}_{3}$ (trigonal)}

Powder data cards. None.

Additional published patterns. None.

NBS sample. The sample of antimony telluride was obtained from Semitronics, Inc., Winchester, Mass. It was heated at $450{ }^{\circ} \mathrm{C}$ overnight in an evacuated tube to sharpen the pattern. Spectrographic analysis showed the following major impurities: 0.001 to 0.01 percent each of aluminum and silicon.

The sample was dark gray opaque powder.

The $d$-values of the three strongest lines are: $3.157,2.349$, and $2.130 \AA$.

Structural data. Dönges [1] in 1951 showed that antimony telluride is isomorphous with bismuth telluride, having the space group $D_{3 d}^{5}-$ $\mathrm{R} \overline{3} \mathrm{~m}$ (No. 166 ), and $3\left(\mathrm{Sb}_{2} \mathrm{Te}_{3}\right)$ per unit hexagonal cell, or $1\left(\mathrm{Sb}_{2} \mathrm{Te}_{3}\right)$ per unit rhombohedral cell. The unit cell measurements reported by Semiletov [2] have been converted from $\mathrm{kX}$ to angstrom units for comparison with NBS values.

Lattice constants

\begin{tabular}{|c|c|c|c|}
\hline \multirow{4}{*}{$\begin{array}{l}1951 \\
1956 \\
1963\end{array}$} & \multirow{4}{*}{$\begin{array}{l}\text { Dönges }[1] \\
\text { Semiletov }[2] \text {. } \\
\text { National Bureau of Stand- } \\
\text { ards at } 25{ }^{\circ} \mathrm{C} \text {. }\end{array}$} & $a$ & $c$ \\
\hline & & $\begin{array}{c}\stackrel{\AA}{\AA} \\
4.25\end{array}$ & $\begin{array}{c}\AA \\
30.35\end{array}$ \\
\hline & & 4. 25 & 29. 92 \\
\hline & & 4. 262 & 30.450 \\
\hline
\end{tabular}

The density of antimony telluride claculated from NBS lattice constants is $6.513 \mathrm{~g} / \mathrm{cm}^{3}$ at $25^{\circ} \mathrm{C}$.

\section{References}

[1] E. Dönges, Ǔber Chalkogenohalogenide des dreiwertigen Antimons und Wismuts. III. Über Tellurohalogenide des dreiwertigen Antimons und Wismuts und über Antimon und Wismut (III) - Tellurid und Wismut (III)-Selenid, Z. anorg. u. Allgem, Chem. 265, 56-61 (1951).

[2] S. A. Semiletov, Electronographic determination of antimony telluride structure, Kristallografiya 1, 403-406 (1956).

\begin{tabular}{|c|c|c|}
\hline \multirow{2}{*}{$\begin{array}{c}h k l \\
\text { (hex.) }\end{array}$} & \multicolumn{2}{|c|}{$\begin{array}{l}\text { Internal Standard, } \\
\text { Tungsten, } a=3.1648 \AA \\
\mathrm{Cu}, 1.5405 \AA \text { at } 25^{\circ} \mathrm{C}\end{array}$} \\
\hline & $d$ & $I$ \\
\hline $\begin{array}{l}003 \\
006 \\
009 \\
104 \\
015\end{array}$ & $\begin{array}{l}\text { A } \\
\text { 10. } 16 \\
\text { 5. } 08 \\
\text { 3. } 383 \\
\text { 3. } 321 \\
\text { 3. } 157\end{array}$ & $\begin{array}{r}1 \\
3 \\
5 \\
1 \\
100\end{array}$ \\
\hline $\begin{array}{c}107 \\
018 \\
1 \cdot 0 \cdot 10 \\
0 \cdot 1 \cdot 11 \\
110\end{array}$ & $\begin{array}{l}\text { 2. } 815 \\
2.651 \\
2.349 \\
2.215 \\
2.130\end{array}$ & $\begin{array}{r}<1 \\
1 \\
34 \\
5 \\
23\end{array}$ \\
\hline $\begin{array}{c}0 \cdot 0 \cdot 15 \\
1 \cdot 0 \cdot 13 \\
116 \\
0 \cdot 1 \cdot 14 \\
119\end{array}$ & $\begin{array}{l}\text { 2. } 030 \\
1.977 \\
1.964 \\
1.875 \\
1.804\end{array}$ & $\begin{array}{l}4 \\
4 \\
1 \\
3 \\
2\end{array}$ \\
\hline $\begin{array}{c}205 \\
0 \cdot 0 \cdot 18 \\
208 \\
0 \cdot 1 \cdot 17 \\
0 \cdot 2 \cdot 10\end{array}$ & $\begin{array}{l}1.766 \\
1.692 \\
1.661 \\
1.611 \\
1.578\end{array}$ & $\begin{array}{r}10 \\
2 \\
<1 \\
2 \\
7\end{array}$ \\
\hline $\begin{array}{c}2 \cdot 0 \cdot 11 \\
1 \cdot 0 \cdot 19,1 \cdot 1 \cdot 15 \\
0 \cdot 0 \cdot 21 \\
0 \cdot 1 \cdot 20 \\
125\end{array}$ & $\begin{array}{l}\text { 1. } 537 \\
\text { 1. } 470 \\
\text { 1. } 450 \\
\text { 1. } 408 \\
\text { 1. } 3597\end{array}$ & $\begin{array}{l}1 \\
7 \\
2 \\
1 \\
7\end{array}$ \\
\hline $\begin{array}{c}1 \cdot 1 \cdot 18 \\
0 \cdot 0 \cdot 24,2 \cdot 1 \cdot 10 \\
0 \cdot 1 \cdot 23 \\
300 \\
0 \cdot 2 \cdot 19\end{array}$ & $\begin{array}{l}\text { 1. } 3249 \\
\text { 1. } 2683 \\
\text { 1. } 2462 \\
\text { 1. } 2303 \\
\text { 1. } 2102\end{array}$ & $\begin{array}{l}1 \\
5 \\
2 \\
1 \\
2\end{array}$ \\
\hline $\begin{array}{c}1 \cdot 1 \cdot 21 \\
2 \cdot 0 \cdot 20,1 \cdot 2 \cdot 14 \\
1 \cdot 0 \cdot 25 \\
2 \cdot 1 \cdot 16 \\
2 \cdot 0 \cdot 23\end{array}$ & $\begin{array}{l}\text { 1. } 1988 \\
\text { 1. } 1742 \\
\text { 1. } 1563 \\
\text { 1. } 1252 \\
\text { 1. } 0759\end{array}$ & $\begin{array}{l}<1 \\
1 \\
<1 \\
<1 \\
<1\end{array}$ \\
\hline $\begin{array}{c}220 \\
2 \cdot 1 \cdot 19 \\
1 \cdot 0 \cdot 28 \\
1 \cdot 2 \cdot 20 \\
0 \cdot 2 \cdot 25\end{array}$ & $\begin{array}{l}\text { 1. } 0655 \\
\text { 1. } 0522 \\
\text { 1. } 0431 \\
\text { 1. } 0286 \\
\text { 1. } 0167\end{array}$ & $\begin{array}{l}1 \\
<1 \\
<1 \\
1 \\
1\end{array}$ \\
\hline $\begin{array}{l}0 \cdot 1 \cdot 29 \\
3 \cdot 0 \cdot 18 \\
1 \cdot 3 \cdot 10 \\
2 \cdot 2 \cdot 15\end{array}$ & $\begin{array}{r}1.0096 \\
0.9952 \\
.9704 \\
.9434\end{array}$ & $\begin{array}{r}1 \\
<1 \\
1 \\
<1\end{array}$ \\
\hline
\end{tabular}




\section{Arsenic Trioxide, claudetite, $\mathrm{As}_{2} \mathrm{O}_{3}$ (monoclinic)}

Powder data cards. None.

Additional published patterns. None.

NBS sample. The sample of claudetite was obtained from the National Museum, No. 1737 from the San Domingo mines, Portugal. Spectrographic analysis showed the following major impurities: 0.1 to 1.0 percent each of aluminum, yttrium, and zinc; 0.01 to 0.1 percent each of barium, calcium, cobalt, chromium, iron, magnesium, titanium, and tungsten.

The sample was colorless. Due to the high indices of refraction a complete optical analysis was not obtained, but partial results seemed to confirm the data given in Dana [4].

The $d$-values of the three strongest lines are: $3.245,3.454$, and $2.771 . \AA$

Structural data. Buerger [1] in 1942 determined that claudetite has the space group $\mathrm{C}_{2 \mathrm{~h}}^{5}-\mathrm{P}_{2} / \mathrm{n}$ (No. 14) and $4\left(\mathrm{As}_{2} \mathrm{O}_{3}\right)$ per unit cell.

The density of claudetite calculated from the NBS lattice constants is $4.186 \mathrm{~g} / \mathrm{cm}^{3}$ at $25{ }^{\circ} \mathrm{C}$.

\section{References}

[1] M. J. Buerger, The unit cell and space group of claudetite, $\mathrm{As}_{2} \mathrm{O}_{3}, \mathrm{Am}$. Mineralogist 27,216 (1942).

[2] K. A. Becker, K. Plieth, and I. N. Stranski, Strukturuntersuchung der monoklinen Arsenikmodifikation Claudetit, Z. anorg. allgem. Chem. 266, 293-301 (1951).

[3] A. J. Frueh, The crystal structure of claudetite (monoclinic $\mathrm{As}_{2} \mathrm{O}_{3}$ ) Am. Mineralogist 36, 833-850 (1951).

[4] Palache, Berman, and Frondel, Dana's System of Mineralogy, 7th Ed. 1, 546 (1951).

\begin{tabular}{|c|c|c|}
\hline \multirow[t]{2}{*}{$h k k l$} & \multicolumn{2}{|c|}{$\begin{array}{c}\text { Internal Standard } \\
\text { Silver, } a=4.0861 \AA \\
\mathrm{Cu}, 1.5405 \AA \text { at } 25{ }^{\circ} \mathrm{C}\end{array}$} \\
\hline & $d$ & $I$ \\
\hline $\begin{array}{l}020 \\
110 \\
011 \\
120 \\
021\end{array}$ & 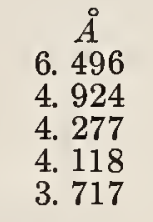 & $\begin{array}{r}9 \\
26 \\
4 \\
9 \\
<3\end{array}$ \\
\hline $\begin{array}{l}\overline{1} 01 \\
\overline{1} 11 \\
130 \\
101 \\
040\end{array}$ & $\begin{array}{l}\text { 3. } 586 \\
\text { 3. } 454 \\
\text { 3. } 356 \\
\text { 3. } 328 \\
\text { 3. } 245\end{array}$ & $\begin{array}{r}14 \\
50 \\
20 \\
18 \\
100\end{array}$ \\
\hline $\begin{array}{c}031 \\
140 \\
131,041 \\
210 \\
\overline{2} 11,150\end{array}$ & $\begin{array}{l}\text { 3. } 129 \\
2.771 \\
2.640 \\
2.608 \\
2,333\end{array}$ & $\begin{array}{r}5 \\
34 \\
15 \\
14 \\
7\end{array}$ \\
\hline $\begin{array}{c}002 \\
051 \\
012 \\
221,151 \\
151\end{array}$ & $\begin{array}{l}\text { 2. } 264 \\
2.253 \\
\text { 2. } 231 \\
\text { 2. } 104 \\
2.048\end{array}$ & $\begin{array}{r}24 \\
5 \\
11 \\
6 \\
5\end{array}$ \\
\hline $\begin{array}{c}112,160 \\
042 \\
\overline{1} 42 \\
\overline{2} 51,170 \\
071\end{array}$ & $\begin{array}{l}\text { 2. } 005 \\
\text { 1. } 857 \\
\text { 1. } 788 \\
\text { 1. } 7514 \\
\text { 1. } 7163\end{array}$ & $\begin{array}{r}<3 \\
11 \\
4 \\
5 \\
8\end{array}$ \\
\hline $\begin{array}{c}\overline{1} 71 \\
080 \\
152, \overline{2} 61 \\
180 \\
\overline{1} 13\end{array}$ & $\begin{array}{l}\text { 1. } 6477 \\
\text { 1. } 6228 \\
\text { 1. } 5988 \\
\text { 1. } 5522 \\
\text { 1. } 4716\end{array}$ & $\begin{array}{r}4 \\
<3 \\
<3 \\
7 \\
4\end{array}$ \\
\hline $\begin{array}{c}123 \\
\overline{3} 32,091 \\
\overline{1} 82 \\
213 \\
\overline{1} 63\end{array}$ & $\begin{array}{l}\text { 1. } 3915 \\
\text { 1. } 3749 \\
\text { 1. } 2937 \\
\text { 1. } 2667 \\
\text { 1. } 2220\end{array}$ & $\begin{array}{r}<3 \\
4 \\
3 \\
<3 \\
<3\end{array}$ \\
\hline$\overline{3} 72,0.11 \cdot 1$ & 1. 1423 & $<3$ \\
\hline
\end{tabular}

Lattice constants

\begin{tabular}{|c|c|c|c|c|c|}
\hline & & $a$ & $b$ & c & $\beta$ \\
\hline $\begin{array}{l}1951 \\
1951 \\
1963\end{array}$ & $\begin{array}{l}\text { Becker, Plieth and Stranski [2] } \\
\text { Frueh [3] } \\
\text { National Bureau of Standards at } 25^{\circ} \mathrm{C}_{-}\end{array}$ & $\begin{array}{l}\stackrel{\AA}{\AA} \\
5.25 \\
5.25 \\
5.339 \\
\pm .002\end{array}$ & $\begin{array}{c}\AA \\
12.90 \\
12.87 \\
12.984 \\
\pm .001\end{array}$ & \begin{tabular}{l}
\multicolumn{1}{c}{} \\
4.53 \\
4.54 \\
4.5405 \\
土. 0005
\end{tabular} & $\begin{array}{l}93^{\circ} 53^{\prime} \\
93^{\circ} 49^{\prime} \\
94^{\circ} 16.1^{\prime} \\
\pm .9^{\prime}\end{array}$ \\
\hline
\end{tabular}




\section{Barium Bromide Monohydrate, $\mathrm{BaBr}_{2} \cdot \mathrm{H}_{2} \mathrm{O}$ (orthorhombic)}

Powder data cards. None.

Additional published patterns. None.

NBS sample. The sample of barium bromide monohydrate was prepared from barium bromide dihydrate obtained from City Chemical Co., New York, N.Y. The hydrate was heated overnight at 85 to $90^{\circ} \mathrm{C}$. Spectrographic analysis showed the following major impurities: 0.01 to 0.1 percent each of sodium and strontium; and 0.001 to 0.01 percent each of calcium, magnesium, silicon, and titanium.

The color of the sample was white. The refractive index could not be determined because the sample was too fine.

The $d$-values of the three strongest lines are: $3.170,2.512$, and $2.999 \AA$.

Structural data. Vainstein and Pinsker [1] in 1950 determined hat barium bromide monohydrate has the barium chloride monohydrate structure, the space group $\mathrm{D}_{2 \mathrm{~h}}^{16}-\mathrm{Pbnm}$ (No. 62), and $4\left(\mathrm{BaBr}_{2} \cdot \mathrm{H}_{2} \mathrm{O}\right)$ per unit cell.

\section{Lattice constants}

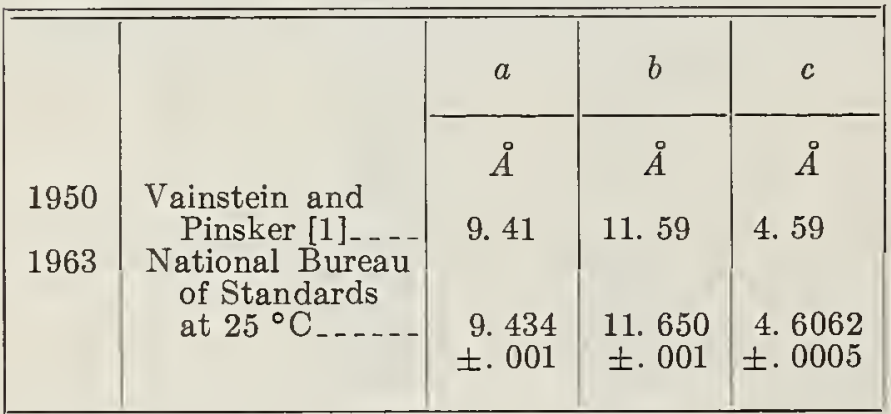

The density of barium bromide monohydrate calculated from the NBS lattice constants is 4.135 $\mathrm{g} / \mathrm{cm}^{3}$ at $25^{\circ} \mathrm{C}$.

\section{Reference}

[1] B. K. Vainstein and Z. G. Pinsker, Electron diffraction study of $\mathrm{BaBr}_{2} \cdot \mathrm{H}_{2} \mathrm{O}$, Zhur. Fiz. Khim. 24, 432 (1950).

\begin{tabular}{|c|c|c|}
\hline \multirow[t]{2}{*}{$h k l$} & \multicolumn{2}{|c|}{$\begin{array}{l}\text { Internal Standard, } \\
\text { Tungsten, } a=3.1648 \AA \\
\mathrm{Cu}, 1.5405 \AA \text { at } 25^{\circ} \mathrm{C}\end{array}$} \\
\hline & $d$ & $I$ \\
\hline $\begin{array}{l}110 \\
020 \\
120 \\
210 \\
101\end{array}$ & $\begin{array}{r}\AA \\
\text { 7. } 36 \\
\text { 5. } 83 \\
\text { 4. } 96 \\
\text { 4. } 38 \\
\text { 4. } 14\end{array}$ & $\begin{array}{r}6 \\
23 \\
5 \\
5 \\
12\end{array}$ \\
\hline $\begin{array}{l}111 \\
021 \\
121 \\
211 \\
230\end{array}$ & $\begin{array}{l}\text { 3. } 91 \\
\text { 3. } 613 \\
\text { 3. } 377 \\
\text { 3. } 170 \\
\text { 2. } 999\end{array}$ & $\begin{array}{r}13 \\
8 \\
2 \\
100 \\
48\end{array}$ \\
\hline $\begin{array}{l}040 \\
131 \\
140 \\
301 \\
231\end{array}$ & $\begin{array}{l}2.913 \\
2.836 \\
2.784 \\
2.596 \\
2.512\end{array}$ & $\begin{array}{r}18 \\
3 \\
4 \\
14 \\
64\end{array}$ \\
\hline $\begin{array}{l}041 \\
400 \\
002 \\
420 \\
022\end{array}$ & $\begin{array}{l}2.462 \\
2.359 \\
2.303 \\
2.189 \\
2.142\end{array}$ & $\begin{array}{r}34 \\
26 \\
26 \\
2 \\
3\end{array}$ \\
\hline $\begin{array}{c}250,122 \\
212 \\
510 \\
232 \\
042\end{array}$ & $\begin{array}{l}2.091 \\
2.038 \\
1.863 \\
1.827 \\
1.807\end{array}$ & $\begin{array}{r}<2 \\
<2 \\
<2 \\
16 \\
4\end{array}$ \\
\hline $\begin{array}{l}061 \\
441 \\
402 \\
270 \\
611\end{array}$ & $\begin{array}{l}\text { 1. } 788 \\
\text { 1. } 703 \\
1.648 \\
1.569 \\
1.476\end{array}$ & $\begin{array}{r}11 \\
12 \\
11 \\
9 \\
3\end{array}$ \\
\hline $\begin{array}{c}080 \\
213,512 \\
442 \\
461 \\
081\end{array}$ & $\begin{array}{l}\text { 1. } 456 \\
\text { 1. } 448 \\
\text { 1. } 434 \\
\text { 1. } 425 \\
\text { 1. } 3882\end{array}$ & $\begin{array}{l}3 \\
6 \\
2 \\
5 \\
4\end{array}$ \\
\hline $\begin{array}{c}303 \\
532 \\
043 \\
323,362 \\
272\end{array}$ & $\begin{array}{l}\text { 1. } 3796 \\
1.3658 \\
1.3582 \\
\text { 1. } 3426 \\
\text { 1. } 2964\end{array}$ & $\begin{array}{r}<2 \\
6 \\
<2 \\
<2 \\
6\end{array}$ \\
\hline $\begin{array}{c}343 \\
082 \\
571,063\end{array}$ & $\begin{array}{l}\text { 1. } 2470 \\
\text { 1. } 2308 \\
\text { 1. } 2041\end{array}$ & $\begin{array}{r}<2 \\
<2 \\
3\end{array}$ \\
\hline
\end{tabular}




\section{Barium Stannate, $\mathrm{BaSnO}_{3}$ (cubic)}

Powder data cards

\begin{tabular}{|c|c|c|}
\hline $\begin{array}{c}\text { Card } \\
\text { number }\end{array}$ & $\begin{array}{c}\text { Index } \\
\text { lines }\end{array}$ & Source \\
\hline $3-0675$ & $\begin{array}{c}2.91 \\
2.68 \\
2.06\end{array}$ & $\begin{array}{c}\text { H. D. Megaw, Philips Lamps } \\
\text { Ltd. }\end{array}$ \\
\hline
\end{tabular}

Additional published patterns. None.

NBS sample. The sample of bariuin stannate was prepared at NBS from stoichiometric amounts of barium carbonate and stannic oxide, pressed into a pellet and heated at $1400{ }^{\circ} \mathrm{C}$ for $1 \mathrm{hr}$. Spectrographic analysis showed the following major impurities: 0.1 percent calcium and 0.01 to 0.1 percent each of aluminum, magnesium, silicon, and strontium.

The sample was very light gray. The indices of refraction could not be determined because the sample was too fine.

The $d$-values of the three strongest lines are: $2.911,1.6805$, and $2.058 \AA$.

Structural data. Megaw [1] in 1946 determined that barium stannate has the perovskite structure, the space group $\mathrm{O}_{\mathrm{h}}^{1}-\mathrm{Pm} 3 \mathrm{~m}$ (No. 221), and 1 $\left(\mathrm{BaSnO}_{3}\right)$ per unit cell.

\section{Lattice constants}

\begin{tabular}{|c|c|c|}
\hline $\begin{array}{l}1946 \\
1957 \\
1958 \\
1963\end{array}$ & $\begin{array}{l}\text { Megaw [1] } \\
\text { Roth [2] } \\
\text { Wagner and Binder [3] } \\
\text { National Bureau of Standards } \\
\quad \text { at } 25^{\circ} \mathrm{C}\end{array}$ & $\begin{array}{l}\stackrel{\AA}{A} \\
\text { 4. } 1168 \\
\text { 4. } 114 \\
\text { 4. } 1157 \\
\text { 4. } 1163\end{array}$ \\
\hline
\end{tabular}

The density of barium stannate calculated from the NBS lattice constant is $7.238 \mathrm{~g} / \mathrm{cm}^{3}$ at $25^{\circ} \mathrm{C}$.

\begin{tabular}{|c|c|c|c|}
\hline \multirow[t]{2}{*}{$h k l$} & \multicolumn{3}{|c|}{$\begin{array}{l}\text { Internal Standard, } \\
\text { Tungsten, } a=3.1648 \AA \\
\mathrm{Cu}, 1.5405 \AA \text { at } 25{ }^{\circ} \mathrm{C}\end{array}$} \\
\hline & $d$ & $I$ & $a$ \\
\hline $\begin{array}{l}110 \\
111 \\
200 \\
211 \\
220\end{array}$ & $\begin{array}{l}\quad \stackrel{\AA}{ } \\
\text { 2. } 911 \\
2.376 \\
2.058 \\
\text { 1. } 6805 \\
1.4555\end{array}$ & $\begin{array}{r}100 \\
4 \\
31 \\
35 \\
16\end{array}$ & 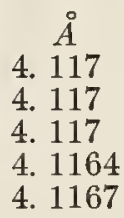 \\
\hline $\begin{array}{l}310 \\
222 \\
321 \\
400 \\
411\end{array}$ & $\begin{array}{l}\text { 1. } 3017 \\
\text { 1. } 1882 \\
\text { 1. } 1002 \\
\text { 1. } 0291 \\
0.9702\end{array}$ & $\begin{array}{r}13 \\
5 \\
13 \\
3 \\
7\end{array}$ & $\begin{array}{l}\text { 4. } 1163 \\
\text { 4. } 1160 \\
\text { 4. } 1166 \\
\text { 4. } 1164 \\
\text { 4. } 1161\end{array}$ \\
\hline $\begin{array}{l}420 \\
332 \\
422 \\
510\end{array}$ & $\begin{array}{l}.9204 \\
.8776 \\
.8402 \\
.8073\end{array}$ & $\begin{array}{r}6 \\
5 \\
5 \\
15\end{array}$ & $\begin{array}{l}\text { 4. } 1162 \\
\text { 4. } 1163 \\
\text { 4. } 1163 \\
\text { 4. } 1164\end{array}$ \\
\hline $\begin{array}{r}\text { Ave } \\
\text { fi }\end{array}$ & $\begin{array}{r}\text { value } \\
\text { hes_. }\end{array}$ & last & 4. 1163 \\
\hline
\end{tabular}

\section{References}

[1] H. D. Megaw, Crystal structure of double oxides of the perovskite type, Proc. Phys. Soc. London 58, 133 (1946)

[2] R. S. Roth, Classification of perovskite and other $\mathrm{ABO}_{3}$-type compounds, J. Res. NBS 58, 75-88 (1957).

[3] G. Wagner and $\mathrm{H}$. Binder, The binary systems $\mathrm{BaO}-\mathrm{SnO}_{2}$ and $\mathrm{BaO}-\mathrm{PbO}_{2}$, II. Crystal structure determinations, Z. anorg. allgem. Chem. 298, 12-21 (1958).

\section{Bismuth Orthophosphate, $\mathrm{BiPO}_{4}$ (monoclinic)}

Powder data cards

\begin{tabular}{|c|c|c|}
\hline $\begin{array}{c}\text { Card } \\
\text { number }\end{array}$ & $\begin{array}{c}\text { Index } \\
\text { lines }\end{array}$ & Source \\
\cline { 2 - 3 } $1-0812$ & $\begin{array}{r}3.08 \\
2.87 \\
4.20\end{array}$ & $\begin{array}{l}\text { Hanawalt, Rinn, and Frevel [1] } \\
1938 .\end{array}$ \\
\hline
\end{tabular}

\section{Additional published patterns. Zemann [2]} 1950.

NBS sample. The sample of monoclinic bismuth orthophosphate was obtained from Bios Laboratories, Inc., New York, N.Y. Speçtrographic analysis showed the following major impurities: 0.1 to 1.0 percent each of calcium and sodium and 0.01 to 0.1 percent each of silver, aluminum, copper, iron, magnesium, lead, and silicon.

The color of the sample was white. The indices of refraction could not be obtained because the sample was too fine.

The $d$-values of the three strongest lines are: $3.066,2.862$, and $4.156 \AA$.

Structural data. Zemann [2] in 1950 determined that monoclinic bismuth orthophosphate has the monazite structure, the space group $\mathrm{C}_{2 \mathrm{~h}}^{5}-$ $\mathrm{P}_{2} /$ n (No. 14), with $4\left(\mathrm{BiPO}_{4}\right)$ per unit cell. According to Mooney-Slater [3] 1962, three forms of bismuth phosphate exist: a hexagonal low phase formed from precipitation at room temperature, a high monoclinic phase formed about $700{ }^{\circ} \mathrm{C}$ and the monoclinic monazite phase formed above $350{ }^{\circ} \mathrm{C}$. 
Bismuth Orthophosphate, $\mathrm{BiPO}_{4}$ (monoclinic)-Continued

Lattice constants

\begin{tabular}{|c|c|c|c|c|c|}
\hline & & $a$ & $b$ & $c$ & $\beta$ \\
\hline $\begin{array}{l}1950 \\
1963\end{array}$ & $\begin{array}{l}\text { Zemann [2] } \\
\text { National Bureau of Standards at } 25^{\circ} \mathrm{C}-\end{array}$ & 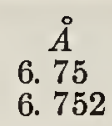 & 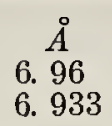 & 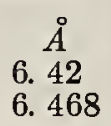 & $\begin{array}{l}104^{\circ} \\
103^{\circ} 42.5^{\prime}\end{array}$ \\
\hline
\end{tabular}

\begin{tabular}{|c|c|c|}
\hline \multirow[t]{2}{*}{$h k l$} & \multicolumn{2}{|c|}{$\begin{array}{l}\text { Internal Standard, } \\
\text { Tungsten, } a=3.1648 \AA \\
\mathrm{Cu}, 1.5405 \AA \text { at } 25{ }^{\circ} \mathrm{C}\end{array}$} \\
\hline & $d$ & $I$ \\
\hline $\begin{array}{l}101 \\
110 \\
011 \\
111 \\
101\end{array}$ & $\begin{array}{l}\stackrel{\circ}{A} \\
\text { 5. } 18 \\
\text { 4. } 761 \\
\text { 4. } 655 \\
\text { 4. } 156 \\
\text { 4. } 079\end{array}$ & $\begin{array}{r}8 \\
12 \\
50 \\
58 \\
22\end{array}$ \\
\hline $\begin{array}{l}111 \\
020 \\
200 \\
002 \\
120\end{array}$ & $\begin{array}{l}3.514 \\
3.466 \\
3.282 \\
3.142 \\
3.066\end{array}$ & $\begin{array}{r}23 \\
8 \\
57 \\
13 \\
100\end{array}$ \\
\hline $\begin{array}{l}021 \\
210 \\
211 \\
012 \\
202\end{array}$ & $\begin{array}{l}\text { 3. } 036 \\
\text { 2. } 963 \\
\text { 2. } 934 \\
\text { 2. } 862 \\
\text { 2. } 597\end{array}$ & $\begin{array}{l}19 \\
19 \\
16 \\
73 \\
22\end{array}$ \\
\hline $\begin{array}{l}211 \\
112 \\
212 \\
220 \\
022\end{array}$ & $\begin{array}{l}\text { 2. } 484 \\
\text { 2. } 438 \\
\text { 2. } 433 \\
\text { 2. } 382 \\
\text { 2. } 328\end{array}$ & $\begin{array}{r}3 \\
18 \\
16 \\
4 \\
11\end{array}$ \\
\hline $\begin{array}{l}130 \\
031 \\
103 \\
\frac{1}{3} 11 \\
131\end{array}$ & $\begin{array}{ll}2 . & 180 \\
2 . & 170 \\
2 . & 150 \\
2 . & 128 \\
2 . & 112\end{array}$ & $\begin{array}{r}3 \\
23 \\
23 \\
18 \\
23\end{array}$ \\
\hline $\begin{array}{l}310 \\
131 \\
212 \\
\frac{3}{2} 13\end{array}$ & $\begin{array}{l}\text { 2. } 085 \\
\text { 2. } 013 \\
\text { 1. } 959 \\
\text { 1. } 929 \\
\text { 1. } 916\end{array}$ & $\begin{array}{r}7 \\
5 \\
24 \\
9 \\
8\end{array}$ \\
\hline
\end{tabular}

\begin{tabular}{|c|c|c|}
\hline \multirow[t]{2}{*}{$h k l$} & \multicolumn{2}{|c|}{$\begin{array}{l}\text { Internal Standard, } \\
\text { Tungsten, } a=3.1648 \AA \\
\mathrm{Cu}, 1.5405 \AA \text { at } 25^{\circ} \mathrm{C}\end{array}$} \\
\hline & $d$ & $I$ \\
\hline $\begin{array}{l}230 \\
231 \\
103 \\
032 \\
320\end{array}$ & $\begin{array}{c}\AA \\
1.889 \\
1.882 \\
1.872 \\
1.862 \\
1.850\end{array}$ & $\begin{array}{r}5 \\
15 \\
6 \\
20 \\
13\end{array}$ \\
\hline $\begin{array}{l}023 \\
\overline{3} 22 \\
231 \\
040 \\
132\end{array}$ & $\begin{array}{l}\text { 1. } 794 \\
\text { 1. } 755 \\
\text { 1. } 745 \\
\text { 1. } 733 \\
\text { 1. } 729\end{array}$ & $\begin{array}{l}14 \\
21 \\
13 \\
14 \\
28\end{array}$ \\
\hline $\begin{array}{l}\overline{3} 13 \\
140 \\
400 \\
\overline{4} 02 \\
\overline{3} 13\end{array}$ & $\begin{array}{l}\text { 1. } 679 \\
\text { 1. } 676 \\
\text { 1. } 640 \\
\text { 1. } 620 \\
\text { 1. } 608\end{array}$ & $\begin{array}{l}5 \\
7 \\
6 \\
8 \\
3\end{array}$ \\
\hline $\begin{array}{l}410 \\
330 \\
312 \\
412 \\
240\end{array}$ & $\begin{array}{l}\text { 1. } 596 \\
\text { 1. } 589 \\
\text { 1. } 581 \\
\text { 1. } 576 \\
\text { 1. } 533\end{array}$ & $\begin{array}{r}9 \\
4 \\
7 \\
6 \\
15\end{array}$ \\
\hline $\begin{array}{c}\overline{2} 14 \\
042 \\
411 \\
133,223 \\
\overline{2} 42\end{array}$ & $\begin{array}{l}\text { 1. } 530 \\
\text { 1. } 518 \\
\text { 1. } 467 \\
\text { 1. } 455 \\
\text { 1. } 442\end{array}$ & $\begin{array}{r}15 \\
3 \\
2 \\
6 \\
4\end{array}$ \\
\hline $\begin{array}{l}\overline{3} 14 \\
421 \\
\overline{4} 31 \\
303 \\
\overline{4} 23 \\
\overline{5} 01\end{array}$ & $\begin{array}{l}\text { 1. } 418 \\
\text { 1. } 379 \\
\text { 1. } 363 \\
\text { 1. } 360 \\
\text { 1. } 355 \\
\text { 1. } 350\end{array}$ & $\begin{array}{l}4 \\
7 \\
7 \\
3 \\
2 \\
5\end{array}$ \\
\hline
\end{tabular}

\section{References}

The density of monoclinic bismuth orthophosphate calculated from NBS lattice constants is $6.863 \mathrm{~g} / \mathrm{cm}^{3}$ at $25^{\circ} \mathrm{C}$.
[1] J. D. Hanawalt, H. W. Rinn, and L. K. Frevel, Chemical analysis by $\mathrm{x}$-ray diffraction, Ind. Eng. Chem. Anal. Ed. 10, 457-512 (1938).

[2] J. Zemann, Beitrage zue Kristallchamie des Wismuts, Tschermaks mineralog. petrog. Mitt. 1, 361-377 (1950).

[3] R. C. L. Mooney-Slater, Polymorphic forms of bismuth phosphate, Z. Krist. 117, 371-385 (1962). 
Powder data cards. None.

Additional published patterns. None.

NBS sample. The sample of trigonal bismuth orthophosphate was precipitated at NBS from a mixture of bismuth nitrate and phosphoric acid. Spectrographic analysis showed the following major impurities: 0.01 to 0.1 percent sodium and 0.001 to 0.01 percent silicon.

The sample was colorless. The indices of refraction could not be determined because the sample was too fine.

The $d$-values of the three strongest lines are: $4.421,3.025$, and $2.854 \AA$.

Structural data. Mooney-Slater [1] in 1962 determined the structure of the trigonal bismuth orthophosphate; it has the space group $\mathrm{D}_{3}^{4}-\mathrm{P} 3_{1} 21$ (No. 152) and $3\left(\mathrm{BiPO}_{4}\right)$ per unit cell. This form is transformed to a monoclinic monazite structure at $350{ }^{\circ} \mathrm{C}$ and to another higher temperature monoclinic form above $700{ }^{\circ} \mathrm{C}$.

Lattice constants

\begin{tabular}{|c|c|c|c|}
\hline \multirow{3}{*}{$\begin{array}{l}1962 \\
1963\end{array}$} & \multirow{3}{*}{$\begin{array}{l}\text { Mooney-Slater }[1] \\
\text { National Bureau of } \\
\text { Standards at } 25^{\circ} \mathrm{C}\end{array}$} & $a$ & $c$ \\
\hline & & $\stackrel{\stackrel{̊}{A}}{6.966}$ & $\begin{array}{c}\stackrel{\circ}{4} \\
6.460\end{array}$ \\
\hline & & $\begin{array}{r}6.9820 \\
\pm 0.0004\end{array}$ & $\begin{array}{r}6.4764 \\
\pm 0.0007\end{array}$ \\
\hline
\end{tabular}

The density of trigonal bismuth orthophosphate calculated from NBS lattice constants is 5.538 $\mathrm{g} / \mathrm{cm}^{3}$ at $25{ }^{\circ} \mathrm{C}$.

\section{Reference}

[1] R.C.L. Mooney-Slater, Polymorphic forms of bismuth phosphate, Z. Krist. 117, 371-385 (1962).

\begin{tabular}{|c|c|c|}
\hline \multirow[t]{2}{*}{$h k l$} & \multicolumn{2}{|c|}{$\begin{array}{l}\text { Internal Standard, } \\
\text { Tungsten, } a=3.1648 \AA \\
\mathrm{Cu}, 1.5405 \AA \text { at } 25^{\circ} \mathrm{C}\end{array}$} \\
\hline & $d$ & $I$ \\
\hline $\begin{array}{l}100 \\
101 \\
110 \\
111 \\
200\end{array}$ & $\begin{array}{l}\stackrel{\AA}{A} \\
\text { 6. } 06 \\
\text { 4. } 421 \\
\text { 3. } 494 \\
\text { 3. } 073 \\
\text { 3. } 025\end{array}$ & $\begin{array}{r}65 \\
100 \\
43 \\
44 \\
94\end{array}$ \\
\hline $\begin{array}{l}102 \\
112 \\
210 \\
202 \\
211\end{array}$ & $\begin{array}{l}\text { 2. } 854 \\
\text { 2. } 375 \\
\text { 2. } 286 \\
\text { 2. } 211 \\
\text { 2. } 156\end{array}$ & $\begin{array}{r}82 \\
32 \\
12 \\
5 \\
61\end{array}$ \\
\hline $\begin{array}{l}103 \\
300 \\
301 \\
212 \\
113\end{array}$ & $\begin{array}{l}\text { 2. } 032 \\
2.016 \\
1.925 \\
1.867 \\
1.837\end{array}$ & $\begin{array}{r}6 \\
4 \\
22 \\
51 \\
6\end{array}$ \\
\hline $\begin{array}{l}203 \\
220 \\
302 \\
310 \\
311\end{array}$ & $\begin{array}{l}\text { 1. } 7571 \\
\text { 1. } 7452 \\
\text { 1. } 7115 \\
\text { 1. } 6774 \\
\text { 1. } 6239\end{array}$ & $\begin{array}{l}24 \\
16 \\
20 \\
12 \\
11\end{array}$ \\
\hline $\begin{array}{l}213 \\
104 \\
222 \\
400 \\
312\end{array}$ & $\begin{array}{l}\text { 1. } 5693 \\
\text { 1. } 5644 \\
1.5368 \\
\text { 1. } 5118 \\
\text { 1. } 4892\end{array}$ & $\begin{array}{r}6 \\
11 \\
5 \\
5 \\
12\end{array}$ \\
\hline $\begin{array}{l}114 \\
402 \\
321 \\
214 \\
322\end{array}$ & $\begin{array}{l}\text { 1. } 4687 \\
\text { 1. } 3698 \\
\text { 1. } 3563 \\
\text { 1. } 3206 \\
\text { 1. } 2750\end{array}$ & $\begin{array}{r}10 \\
3 \\
14 \\
15 \\
10\end{array}$ \\
\hline $\begin{array}{l}105 \\
304 \\
403 \\
412 \\
501\end{array}$ & $\begin{array}{l}\text { 1. } 2667 \\
\text { 1. } 2624 \\
\text { 1. } 2384 \\
\text { 1. } 2217 \\
\text { 1. } 1885\end{array}$ & $\begin{array}{l}\mathbf{5} \\
7 \\
2 \\
\mathbf{5} \\
\mathbf{5}\end{array}$ \\
\hline $\begin{array}{l}330 \\
331 \\
420 \\
502 \\
215\end{array}$ & $\begin{array}{l}\text { 1. } 1639 \\
\text { 1. } 1450 \\
\text { 1. } 1425 \\
\text { 1. } 1328 \\
\text { 1. } 1267\end{array}$ & $\begin{array}{l}8 \\
3 \\
4 \\
5 \\
7\end{array}$ \\
\hline $\begin{array}{c}413,421 \\
510 \\
422 \\
511\end{array}$ & $\begin{array}{l}\text { 1. } 1255 \\
\text { 1. } 0860 \\
\text { 1. } 0774 \\
1.0709\end{array}$ & $\begin{array}{l}5 \\
4 \\
3 \\
2\end{array}$ \\
\hline
\end{tabular}




\section{Bismuth Orthovanadate (low form), $\mathrm{BiVO}_{4}$ (tetragonal)}

Powder data cards. None. (Card No. 12-293 gives data for a mineral pucherite, $\mathrm{BiVO}_{4}$, which is orthorhombic and does not compare with either of the synthetic forms of $\mathrm{BiVO}_{4}$.)

Additional published patterns. None.

NBS sample. The sample of low form bismuth orthovanadate was obtained from City Chemical Corp., New York, N.Y. Spectrographic analysis showed the following major impurities: 0.001 to 0.01 percent each of aluminum and silicon. The sample was also precipitated at NBS from solutions of $\mathrm{Na}_{3} \mathrm{VO}_{4}$ and $\mathrm{Bi}\left(\mathrm{NO}_{3}\right)_{3}$. If the precipitate is heated below $400{ }^{\circ} \mathrm{C}$, the tetragonal form is unchanged. Between 400 and $500^{\circ} \mathrm{C}$ the changed to the stable monoclinic form occurs.

The color of the sample was orange-yellow. The indices of refraction could not be determined because the sample was too fine.

The $d$-values of the three strongest lines are: $3.649,2.738$, and $1.879 \AA$.

Structural data. The structure of low form bismuth orthovanadate has not been reported in the literature. However, because of the similarity of patterns it is thought to be isostructural with zircon, having the space group $\mathrm{D}_{4 \mathrm{~h}}^{19}-\mathrm{I} 4_{1} /$ amd (No. $141)$ and $4\left(\mathrm{BiVO}_{4}\right)$ per unit cell.

Lattice constants

\begin{tabular}{|c|c|c|c|}
\hline & & $a$ & $c$ \\
\hline 1963 & $\begin{array}{l}\text { National Bureau } \\
\text { of Standards at } \\
26^{\circ} \mathrm{C}\end{array}$ & $\begin{array}{c}\AA \\
\text { 7. } 2999\end{array}$ & $\begin{array}{c}\AA \\
\text { 6. } 4573\end{array}$ \\
\hline
\end{tabular}

The density of low form bismuth orthovanadate calculated from NBS lattice constants is 6.252 $\mathrm{g} / \mathrm{cm}^{3}$ at $26{ }^{\circ} \mathrm{C}$.

\begin{tabular}{|c|c|c|}
\hline \multirow[t]{2}{*}{$h l c l$} & \multicolumn{2}{|c|}{$\begin{array}{l}\text { Internal Standard, } \\
\text { Tungsten, } a 3.1648 \AA \\
\mathrm{Cu}, 1.5405 \AA \text { at } 26^{\circ} \mathrm{C}\end{array}$} \\
\hline & $d$ & $I$ \\
\hline $\begin{array}{l}101 \\
200 \\
211 \\
112 \\
220\end{array}$ & $\begin{array}{c}\stackrel{\AA}{A} \\
\text { 4. } 839 \\
\text { 3. } 649 \\
2.912 \\
\text { 2. } 738 \\
\text { 2. } 582\end{array}$ & $\begin{array}{r}47 \\
100 \\
22 \\
61 \\
21\end{array}$ \\
\hline $\begin{array}{l}202 \\
301 \\
103 \\
321 \\
312\end{array}$ & $\begin{array}{l}2.419 \\
\text { 2. } 278 \\
2.065 \\
\text { 1. } 932 \\
\text { 1. } 879\end{array}$ & $\begin{array}{r}3 \\
18 \\
11 \\
14 \\
48\end{array}$ \\
\hline $\begin{array}{l}400 \\
213 \\
411 \\
420 \\
004\end{array}$ & $\begin{array}{l}1.825 \\
\text { 1. } 797 \\
\text { 1. } 708 \\
\text { 1. } 632 \\
1.614\end{array}$ & $\begin{array}{r}15 \\
7 \\
5 \\
11 \\
7\end{array}$ \\
\hline $\begin{array}{l}332 \\
204 \\
501 \\
224 \\
521\end{array}$ & $\begin{array}{l}\text { 1. } 5183 \\
\text { 1. } 4765 \\
\text { 1. } 4240 \\
\text { 1. } 3687 \\
\text { 1. } 3261\end{array}$ & $\begin{array}{r}10 \\
10 \\
6 \\
11 \\
3\end{array}$ \\
\hline $\begin{array}{l}512 \\
600 \\
404 \\
215 \\
611\end{array}$ & $\begin{array}{l}\text { 1. } 3087 \\
\text { 1. } 2169 \\
\text { 1. } 2092 \\
\text { 1. } 2008 \\
\text { 1. } 1799\end{array}$ & $\begin{array}{r}12 \\
6 \\
9 \\
5 \\
2\end{array}$ \\
\hline $\begin{array}{l}532 \\
620 \\
424\end{array}$ & $\begin{array}{l}\text { 1. } 1673 \\
\text { 1. } 1543 \\
\text { 1. } 1478\end{array}$ & $\begin{array}{l}6 \\
7 \\
9\end{array}$ \\
\hline
\end{tabular}

\section{Bismuth Orthovanadate (high form), $\mathrm{BiVO}_{4}$ (monoclinic)}

Powder data cards. None. (Card No. 12-293 gives data for a mineral pucherite, $\mathrm{BiVO}_{4}$, which is orthorhombic and does not correspond to either form of $\mathrm{BiVO}_{4}$ which we prepared.)

Additional published patterns. None.

NBS sample. The sample of high form bismuth orthovanadate was prepared by R. S. Roth at NBS by heating stoichiometric mixtures of bismuth oxide and vanadium oxide at $895{ }^{\circ} \mathrm{C}$ for 16 hr. Spectrographic analysis showed the following major impurities: 0.001 to 0.01 percent each of aluminum and silicon. The monoclinic form was also prepared from the low form which was made by precipitation from solutions of $\mathrm{Na}_{3} \mathrm{VO}_{4}$ and $\mathrm{Bi}\left(\mathrm{NO}_{3}\right)_{3}$. The low form sample is tetragonal up to approximately $400{ }^{\circ} \mathrm{C}$. The nonreversible change to the monoclinic form takes place between 400 and $500{ }^{\circ} \mathrm{C}$.

The color of the sample was orange-yellow. The indices of refraction could not be determined because the sample was too fine.

The $d$-values of the three strongest lines are: $3.095,3.082$, and $3.120 \AA$.

Structural data. Roth and Waring [1] in 1963 reported that high form $\mathrm{BiVO}_{4}$ is thought to be isostructural with fergusonite because of the similarity of patterns. Ferguson [2] in 1955 reported that the fergusonite structure is monoclinic. The probable space group is $\mathrm{C}_{2 \mathrm{~h}}^{6}-\mathrm{I} 2 / \mathrm{a}$ (No. 15) with $4\left(\mathrm{BiVO}_{4}\right)$ per unit cell. 
Bismuth Orthovanadate (high form), $\mathrm{BiVO}_{4}$ (monoclinic)-Continued

Lattice constants

\begin{tabular}{|c|c|c|c|c|c|}
\hline & & $a$ & $b$ & $c$ & $\beta$ \\
\hline 1963 & National Bureau of Standards at $26{ }^{\circ} \mathrm{C} \ldots \ldots$ & $\begin{array}{c}\stackrel{\circ}{A} \\
\text { 5. } 195\end{array}$ & 11. $\stackrel{\AA}{\AA}$ & $\begin{array}{c}\stackrel{\circ}{A} \\
\text { 5. } 092\end{array}$ & $90.38^{\circ}$ \\
\hline
\end{tabular}

\begin{tabular}{|c|c|c|}
\hline \multirow[t]{2}{*}{$h k l$} & \multicolumn{2}{|c|}{$\begin{array}{c}\text { Internal Standard } \\
\text { Silver, } a=4.0861 \AA \\
\mathrm{Cu}, 1.5405 \AA \text { at } 26^{\circ} \mathrm{C} .\end{array}$} \\
\hline & $d$ & $I$ \\
\hline & $\stackrel{\circ}{\AA}$ & \\
\hline 020 & 5. 847 & 2 \\
\hline 110 & 4. 749 & 23 \\
\hline 011 & 4. 670 & 27 \\
\hline 130 & 3. 120 & 32 \\
\hline 031,121 & 3. 095 & 100 \\
\hline$\overline{1} 21$ & 3. 082 & 96 \\
\hline 040 & 2. 924 & 26 \\
\hline 200 & 2. 598 & 12 \\
\hline 002 & 2. 546 & 14 \\
\hline 220 & 2.374 & 1 \\
\hline 141 & 2. 282 & 6 \\
\hline$\overline{1} 41,211$ & 2. 277 & 6 \\
\hline 211 & 2. 264 & 11 \\
\hline 112 & 2. 250 & 9 \\
\hline$\overline{1} 12$ & 2. 239 & 7 \\
\hline 150 & 2. 133 & 7 \\
\hline 051 & 2. 127 & 11 \\
\hline 231 & 1. 995 & 5 \\
\hline$\overline{2} 31$ & 1. 988 & 3 \\
\hline 132 & 1. 976 & 5 \\
\hline$\overline{1} 32$ & 1. 970 & 7 \\
\hline 060 & 1. 949 & 4 \\
\hline 240 & 1. 943 & 15 \\
\hline 042 & 1. 920 & 16 \\
\hline 202 & 1. 824 & 6 \\
\hline$\overline{2} 02$ & 1. 812 & 8 \\
\hline$\overline{2} 22$ & 1. 726 & 2 \\
\hline 161 & 1. 719 & 18 \\
\hline 161 & 1. 717 & 17 \\
\hline 310 & 1. 713 & 3 \\
\hline 013 & 1. 680 & 3 \\
\hline 251 & 1. 648 & 2 \\
\hline$\overline{2} 51$ & 1. 644 & 3 \\
\hline 152 & 1. 639 & 2 \\
\hline 152 & 1. 633 & 2 \\
\hline 170 & 1. 591 & 4 \\
\hline 071 & 1. 587 & 5 \\
\hline 330,321 & 1. 582 & 10 \\
\hline$\overline{3} 21$ & 1. 5757 & 9 \\
\hline 123 & 1. 5580 & 8 \\
\hline 033 & 1. 5562 & 4 \\
\hline
\end{tabular}

The density of high form bismuth orthovanadate calculated from NBS lattice constants is $6.951 \mathrm{~g} / \mathrm{cm}^{3}$ at $26^{\circ} \mathrm{C}$.

\begin{tabular}{|c|c|c|}
\hline \multirow[t]{2}{*}{$h k l$} & \multicolumn{2}{|c|}{$\begin{array}{c}\text { Internal Standard } \\
\text { Silver, } a=4.0861 \AA \\
\mathrm{Cu}, 1.5405 \AA \text { at } 26^{\circ} \mathrm{C}\end{array}$} \\
\hline & $d$ & $I$ \\
\hline & $\AA$ & \\
\hline$\overline{1} 23$ & 1. 5522 & 7 \\
\hline 062,242 & 1. 5482 & 11 \\
\hline 242 & 1. 5405 & 5 \\
\hline 080 & 1. 4625 & 3 \\
\hline$\overline{3} 12$ & 1. 4167 & 2 \\
\hline 143,213 & 1. 4146 & 2 \\
\hline & 1. 4067 & $<1$ \\
\hline & 1. 3744 & 1 \\
\hline 181,271 & 1. 3566 & 2 \\
\hline$\overline{1} 72$ & 1. 3481 & 3 \\
\hline$\overline{3} 51$ & 1. 3408 & 1 \\
\hline 262 & 1. 3318 & 1 \\
\hline 400 & 1. 2989 & 2 \\
\hline 280 & 1. 2747 & 4 \\
\hline 004 & 1. 2725 & 4 \\
\hline 082 & 1. 2683 & 4 \\
\hline 091 & 1. 2598 & 2 \\
\hline 361 & 1. 2566 & 3 \\
\hline$\overline{3} 61$ & 1. 2536 & 4 \\
\hline 163 & 1. 2447 & 3 \\
\hline 163 & 1. 2417 & 3 \\
\hline$\overline{3} 5 \underline{2}, 253$ & 1. 2175 & 2 \\
\hline$\overline{2} 53$ & 1. 2117 & 1 \\
\hline 370 & 1. 2028 & 1 \\
\hline$\overline{4} 31$ & 1. 1958 & $<1$ \\
\hline 073,323 & 1. 1906 & 2 \\
\hline 440 & 1. 1871 & 3 \\
\hline$\overline{3} 23$ & 1. 1831 & 4 \\
\hline 044 & 1. 1670 & 1 \\
\hline 402 & 1. 1600 & $<1$ \\
\hline 204 & 1. 1461 & 1 \\
\hline 282 & 1. 1411 & 1 \\
\hline
\end{tabular}

\section{References}

[1] R. S. Roth and J. L. Waring, Synthesis and stability of bismutotantalite, stibiotantalite, and chemically similar $\mathrm{ABO}_{4}$ compounds, Am. Mineralogist 48, No. $11,12,1348-1356$ (1963).

[2] R. B Ferguson, The crystallography of synthetic $\mathrm{YTaO}_{4}$ and fused fergusonite, Bull. Geol. Soc. Amer. 66, 1557 (1955). 
Powder data cards

\begin{tabular}{|c|c|c|}
\hline $\begin{array}{c}\text { Card } \\
\text { number }\end{array}$ & $\begin{array}{l}\text { Index } \\
\text { lines }\end{array}$ & Source \\
\hline $\begin{array}{r}8-21 \\
10-54\end{array}$ & $\begin{array}{l}\text { 3. } 22 \\
\text { 2. } 37 \\
\text { 2. } 19 \\
\text { 2. } 03 \\
\text { 3. } 20 \\
\text { 3. } 25\end{array}$ & $\begin{array}{l}\text { Thompson [1]. } \\
\text { Vasenin and Konovalov [2]. }\end{array}$ \\
\hline
\end{tabular}

Additional published patterns. Harcourt [3] 1942.

NBS sample. The sample of bismuth telluride was obtained as a single crystal from Semitronics Inc., Winchester, Mass. Spectrographic analysis showed the following impurities: 0.0001 to 0.001 percent each of magnesium and silicon.

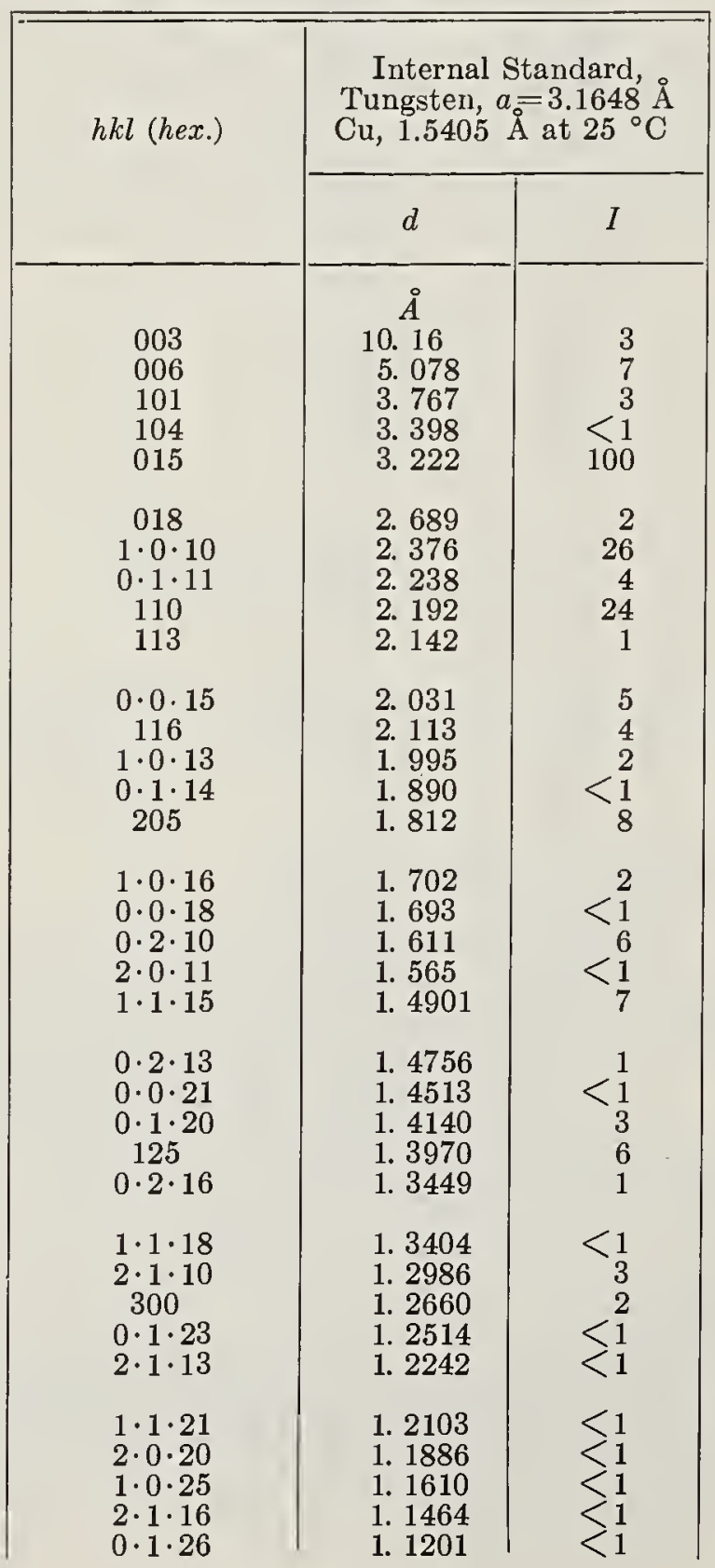

-The sample was a gray opaque powder.

The $d$-values of the three strongest lines are: $3.222,2.376$, and $2.192 \AA$.

Structural data. Lange [4] in 1939 determined that bismuth telluride has the tetradymite structure with the space group $\mathrm{D}_{3 \mathrm{~d}}^{5}-\mathrm{R} \overline{3} \mathrm{~m}$ (No. 166) and $3\left(\mathrm{Bi}_{2} \mathrm{Te}_{3}\right)$ per unit hexagonal cell.

Lattice constants

\begin{tabular}{|c|c|c|c|}
\hline & & $a$ & $c$ \\
\hline $\begin{array}{l}1940 \\
1940 \\
1951 \\
1954 \\
1963\end{array}$ & $\begin{array}{l}\text { Frondel [5] } \\
\text { Peacock and Berry }[6] \\
\text { Dönges }[7]_{-} \\
\text {Semiletov }[8] \\
\text { National Bureau of } \\
\text { Standards at } 25{ }^{\circ} \mathrm{C}_{-}\end{array}$ & $\begin{array}{l}\quad \stackrel{\circ}{A} \\
\text { 4. } 39 \\
\text { 4. } 384 \\
\text { 4. } 35 \\
\text { 4. } 39 \\
\text { 4. } 3852\end{array}$ & $\begin{array}{l}\quad \stackrel{\AA}{A} \\
30.7 \\
30.45 \\
30.3 \\
30.46 \\
\\
30.483\end{array}$ \\
\hline
\end{tabular}

The density of bismuth telluride calculated from the NBS lattice constants is $7.857 \mathrm{~g} / \mathrm{cm}^{3}$ at $25^{\circ} \mathrm{C}$.

\begin{tabular}{|c|c|c|}
\hline \multirow[t]{2}{*}{$h k l$ (hex.) } & \multicolumn{2}{|c|}{$\begin{array}{l}\text { Internal Standard, } \\
\text { Tungsten, } a=3.1648 \AA \\
\mathrm{Cu}, 1.5405 \mathrm{~A} \text { at } 25{ }^{\circ} \mathrm{C}\end{array}$} \\
\hline & $d$ & $I$ \\
\hline $\begin{array}{l}220 \\
2 \cdot 0 \cdot 23 \\
3 \cdot 0 \cdot 15 \\
1 \cdot 0 \cdot 28 \\
315\end{array}$ & $\begin{array}{ll} & \AA \\
\text { 1. } & 0963 \\
1.0868 \\
\text { 1. } 0744 \\
\text { 1. } 0464 \\
\text { 1. } 0379\end{array}$ & $\begin{array}{l}\sum_{1} \\
\sum_{1} \\
1 \\
1\end{array}$ \\
\hline $\begin{array}{l}0 \cdot 2 \cdot 25 \\
0 \cdot 0 \cdot 30 \\
2 \cdot 0 \cdot 26 \\
1 \cdot 3 \cdot 10 \\
1 \cdot 2 \cdot 23\end{array}$ & $\begin{array}{r}1.0261 \\
1.0163 \\
.9978 \\
.9956 \\
.9738\end{array}$ & $\begin{array}{l}<1 \\
\sum 1 \\
<1 \\
2 \\
<1\end{array}$ \\
\hline $\begin{array}{l}2 \cdot 2 \cdot 15 \\
3 \cdot 0 \cdot 21 \\
1 \cdot 0 \cdot 31 \\
0 \cdot 2 \cdot 28 \\
045\end{array}$ & $\begin{array}{l}.9649 \\
.9540 \\
.9518 \\
.9444 \\
.9382\end{array}$ & $\begin{array}{l}\sum_{1} 1 \\
\sum 1 \\
\sum 1\end{array}$ \\
\hline $\begin{array}{c}2 \cdot 1 \cdot 25 \\
0 \cdot 1 \cdot 32,0 \cdot 0 \cdot 33 \\
1 \cdot 2 \cdot 26,3 \cdot 1 \cdot 17 \\
4 \cdot 0 \cdot 10 \\
2 \cdot 2 \cdot 21\end{array}$ & $\begin{array}{l}.9294 \\
.9238 \\
.9080 \\
.9064 \\
.8750\end{array}$ & $\begin{array}{l}\sum_{1} \\
\sum_{1} \\
<1\end{array}$ \\
\hline $\begin{array}{c}2 \cdot 1 \cdot 28 \\
3 \cdot 1 \cdot 20 \\
235 \\
1 \cdot 1 \cdot 33 \\
0 \cdot 1 \cdot 35\end{array}$ & $\begin{array}{l}.8673 \\
.8666 \\
.8626 \\
.8512 \\
.8488\end{array}$ & $\begin{array}{l}2 \\
\sum 1 \\
<1 \\
1\end{array}$ \\
\hline $\begin{array}{c}3 \cdot 2 \cdot 10 \\
410\end{array}$ & $\begin{array}{l}.8377 \\
.8286\end{array}$ & $\sum_{1}$ \\
\hline
\end{tabular}




\section{Bismuth Telluride (tellurobismuthite), $\mathrm{Bi}_{2} \mathrm{Te}_{3}$ (trigonal)—Continued}

\section{References}

[1] R. M. Thompson, The telluride minerals and their occurrence in Canada, Am. Mineralogist 34, 342382 (1949).

[2] F. I. Vasenin and P. F. Konovalov, Ionization x-ray structure investigation of bismuth telluride, J. Tech. Phys. 26, part 7, 1376 (1956).

[3] G. A. Harcourt, Tables for the identification of ore minerals by x-ray powder diffraction, Am. Mineralogist 27, 100 (1942).

[4] P. W. Lange, Ein vergleich zwischen $\mathrm{Bi}_{2} \mathrm{Te}_{3}$ und $\mathrm{Bi}_{2} \mathrm{Te}_{2} \mathrm{~S}$, Naturwissenschaften 27, 133 (1939).
[5] C. Frondel, Redefinition of tellurobismuthite and vandiestite, Am. J. Sci. 238, 880-888 (1940).

[6] M. A. Peacock and L. G. Berry, Röntgenographic observations on ore minerals, Univ. Toronto Studies Geol. Ser. 44-48, 67 (1940-1943).

[7] E. Dönges, Ủber Chalkogenohalogenide dreiwertigen Antimons und Wismuts III. Ưber Tellurohalogenide des dreiwertigen Antimons und Wismuts und über Antimon und Wismut III-Tellurid und Wismut III-Selenid, Z. anorg. allgem. Chem. 265, 56 (1951).

[8] S. A. Semiletov, An electron diffraction study of films of Bi-Se and Bi-Te prepared by evaporation, Trudy Inst. Krist. Akad. Nauk SSSR 10, 76-83 (1954).

\section{Bismuth Trioxide (bismite), alpha $\mathrm{Bi}_{2} \mathrm{O}_{3}$ (pseudo-orthorhombic)}

Powder data cards.

\begin{tabular}{|c|c|c|}
\hline $\begin{array}{c}\text { Card } \\
\text { numbers }\end{array}$ & $\begin{array}{c}\text { Index } \\
\text { lines }\end{array}$ & Source \\
\hline $6-0294$ & $\begin{array}{r}3.26 \\
1.96 \\
1.75\end{array}$ & Sillén [1] 1941. \\
& $\begin{array}{l}3.23 \\
2.68 \\
6-0307\end{array}$ & Frondel [2] 1943. \\
& \\
\hline
\end{tabular}

NBS sample. The sample of bismuth trioxide was obtained from Johnson, Matthey, and Co., Ltd. Their spectrographic analysis showed the following major impurities: less than 0.001 percent each of silicon aluminum, lead, silver, and sodium.

The color of the sample was light yellow. The refractive indices were too high to be determined by the usual liquid-grain immersion method.

The $d$-values of the three strongest lines are: $3.253,2.693$, and $2.708 \AA$.

Structural data. Sillén [1] in 1941 determined that alpha bismuth oxide is monoclinic or pseudoorthorhombic having the monoclinic space group $\mathrm{C}_{2 \mathrm{n}}^{5}-\mathrm{P} 2_{1} / \mathrm{c}$ (No. 14), with $4\left(\mathrm{Bi}_{2} \mathrm{O}_{3}\right)$ per unit monoclinic cell, or $8\left(\mathrm{Bi}_{2} \mathrm{O}_{3}\right)$ per unit pseudo-orthorhombic cell. According to Sillén [1], the monoclinic indices $h k l$ are transformed into the pseudo-orthorhombic indices $h^{\prime} k^{\prime} l^{\prime}$ by the following relations

$$
h^{\prime}=h, k^{\prime}=k, \quad( \pm) l^{\prime}=2 l-h
$$

The lattice constants reported by Sillén have been converted from kX to angstrom units for comparison with the NBS values.

\begin{tabular}{|c|c|c|}
\hline \multirow[t]{2}{*}{$h k l$} & \multicolumn{2}{|c|}{$\begin{array}{l}\text { Internal Standard, } \\
\text { Tungsten, } a=3.1648 \AA \\
\mathrm{Cu}, 1.5405 \AA \text { at } 25^{\circ} \mathrm{C}\end{array}$} \\
\hline & $d$ & $I$ \\
\hline $\begin{array}{l}120 \\
111 \\
200 \\
031 \\
220\end{array}$ & $\begin{array}{l}\stackrel{\AA}{A} \\
\text { 5. } 276 \\
\text { 4. } 498 \\
\text { 4. } 084 \\
\text { 3. } 622 \\
\text { 3. } 517\end{array}$ & $\begin{array}{l}2 \\
4 \\
4 \\
8 \\
2\end{array}$ \\
\hline $\begin{array}{l}040 \\
131 \\
211 \\
140 \\
102\end{array}$ & $\begin{array}{l}\text { 3. } 456 \\
\text { 3. } 310 \\
\text { 3. } 253 \\
\text { 3. } 184 \\
\text { 2. } 753\end{array}$ & $\begin{array}{r}19 \\
33 \\
100 \\
25 \\
6\end{array}$ \\
\hline $\begin{array}{l}231 \\
022 \\
240 \\
122 \\
320\end{array}$ & $\begin{array}{l}\text { 2. } 708 \\
\text { 2. } 693 \\
\text { 2. } 638 \\
\text { 2. } 559 \\
\text { 2. } 532\end{array}$ & $\begin{array}{r}38 \\
39 \\
6 \\
15 \\
9\end{array}$ \\
\hline $\begin{array}{l}051 \\
311 \\
151 \\
222 \\
331\end{array}$ & $\begin{array}{l}\text { 2. } 499 \\
\text { 2. } 429 \\
\text { 2. } 390 \\
\text { 2. } 244 \\
\text { 2. } 176\end{array}$ & $\begin{array}{r}7 \\
6 \\
14 \\
6 \\
6\end{array}$ \\
\hline $\begin{array}{l}142 \\
340 \\
251 \\
400 \\
260\end{array}$ & $\begin{array}{l}\text { 2. } 154 \\
\text { 2. } 138 \\
\text { 2. } 132 \\
\text { 2. } 041 \\
\text { 2. } 0064\end{array}$ & $\begin{array}{l}5 \\
2 \\
8 \\
2 \\
5\end{array}$ \\
\hline $\begin{array}{c}302 \\
242,420 \\
013 \\
322 \\
411\end{array}$ & $\begin{array}{l}1.9922 \\
\text { 1. } 9584 \\
\text { 1. } 9317 \\
\text { 1. } 9136 \\
\text { 1. } 9098\end{array}$ & $\begin{array}{r}4 \\
26 \\
<1 \\
2 \\
4\end{array}$ \\
\hline $\begin{array}{l}113 \\
071 \\
351 \\
171 \\
062\end{array}$ & $\begin{array}{l}\text { 1. } 8787 \\
\text { 1. } 8720 \\
\text { 1. } 8409 \\
\text { 1. } 8237 \\
\text { 1. } 8087\end{array}$ & $\begin{array}{r}9 \\
17 \\
6 \\
7 \\
1\end{array}$ \\
\hline $\begin{array}{l}033 \\
431 \\
162 \\
360 \\
133\end{array}$ & $\begin{array}{l}\text { 1. } 7967 \\
\text { 1. } 7790 \\
\text { 1. } 7660 \\
\text { 1. } 7590 \\
\text { 1. } 7549\end{array}$ & $\begin{array}{r}<1 \\
2 \\
8 \\
9 \\
6\end{array}$ \\
\hline
\end{tabular}


Bismuth Trioxide (bismite), alpha $\mathrm{Bi}_{2} \mathrm{O}_{3}$ (pseudo-orthorhombic)-Continued

\begin{tabular}{|c|c|c|}
\hline \multirow[t]{2}{*}{$h k l$} & \multicolumn{2}{|c|}{$\begin{array}{l}\text { Internal Standard, } \\
\text { Tungsten, } a=3.1648 \AA \\
\mathrm{Cu}, 1.5405 \AA \text { at } 25{ }^{\circ} \mathrm{C}\end{array}$} \\
\hline & $d$ & $I$ \\
\hline $\begin{array}{l}213 \\
342 \\
180 \\
402 \\
262\end{array}$ & $\begin{array}{l}\stackrel{\AA}{A} \\
\text { 1. } 7452 \\
\text { 1. } 7265 \\
\text { 1. } 6914 \\
\text { 1. } 6743 \\
\text { 1. } 6551\end{array}$ & $\begin{array}{r}16 \\
12 \\
1 \\
13 \\
10\end{array}$ \\
\hline $\begin{array}{l}233 \\
422 \\
053 \\
280 \\
451\end{array}$ & $\begin{array}{l}\text { 1. } 6431 \\
\text { 1. } 6273 \\
\text { 1. } 5935 \\
\text { 1. } 5920 \\
1.5815\end{array}$ & $\begin{array}{r}10 \\
2 \\
8 \\
10 \\
7\end{array}$ \\
\hline $\begin{array}{c}313 \\
153,511 \\
442 \\
333 \\
531\end{array}$ & $\begin{array}{l}1.5754 \\
1.5632 \\
1.5065 \\
1.4994 \\
1.4888\end{array}$ & $\begin{array}{l}3 \\
6 \\
6 \\
5 \\
5\end{array}$ \\
\hline $\begin{array}{l}091 \\
004 \\
104 \\
124 \\
282\end{array}$ & $\begin{array}{l}\text { 1. } 4854 \\
\text { 1. } 4625 \\
\text { 1. } 4391 \\
\text { 1. } 4093 \\
\text { 1. } 3983\end{array}$ & $\begin{array}{l}6 \\
5 \\
1 \\
3 \\
6\end{array}$ \\
\hline $\begin{array}{c}073 \\
471 \\
551 \\
1.10 \cdot 0 \\
433 \\
044\end{array}$ & $\begin{array}{l}\text { 1. } 3882 \\
\text { 1. } 3792 \\
\text { 1. } 3672 \\
\text { 1. } 3637 \\
\text { 1. } 3485 \\
\text { 1. } 3462\end{array}$ & $\begin{array}{l}1 \\
3 \\
4 \\
3 \\
3 \\
3\end{array}$ \\
\hline
\end{tabular}

Lattice constants

\begin{tabular}{|c|c|c|c|c|}
\hline \multirow{3}{*}{$\begin{array}{l}1941 \\
1963\end{array}$} & \multirow{3}{*}{$\begin{array}{l}\text { Sillén [1] } \\
\text { National Bureau } \\
\text { of Standards at } \\
25{ }^{\circ} \mathrm{C}\end{array}$} & $a$ & $b$ & $c$ \\
\hline & & $\begin{array}{c}\stackrel{\circ}{A} \\
\text { 8. }\end{array}$ & 13. $\stackrel{\circ}{A} 81$ & $\begin{array}{c}\stackrel{\circ}{A} \\
\text { 5. } 84\end{array}$ \\
\hline & & 8. 166 & 13. 827 & 5. 850 \\
\hline
\end{tabular}

The density of alpha bismuth trioxide calculated from NBS lattice constants is $9.371 \mathrm{~g} / \mathrm{cm}^{3}$ at $25^{\circ} \mathrm{C}$.

\section{References}

[1] L. G. Sillen, On the crystal structure of monoclinic $\alpha-\mathrm{Bi}_{2} \mathrm{O}_{3}, \mathrm{Z}$. Krist. 103, 274-290 (1941).

[2] C. Frondel, Mineralogy of the oxides and carbonates of bismuth, Am. Mineralogist 28, Nos. 9 and 10 . 521-535 (1943). 


\section{Cadmium Perchlorate Hexahydrate, $\mathrm{Cd}\left(\mathrm{ClO}_{4}\right)_{2} \cdot 6 \mathrm{H}_{2} \mathrm{O}$ (trigonal)}

Powder data cards. None.

Additional published patterns. None.

NBS sample. The sample of cadmium perchlorate hexahydrate was obtained from the City Chemical Corp., New York, N.Y. Spectrographic analysis showed the following major impurities: 0.0001 to 0.001 percent each of lead and silicon:

The sample was colorless and optically negative with indices of refraction $\mathrm{N}_{0}=1.490$ and $\mathrm{N}_{\mathrm{e}}=$ 1.479 .

The $d$-values of the three strongest lines are: $3.995,4.223$, and $2.902 \AA$.

Structural data. West [1] in 1935 reported that cadmium perchlorate hexahydrate is trigonal, the space group $\mathrm{C}_{3 \mathrm{v}}^{1}-\mathrm{P} 3 \mathrm{ml}(\mathrm{No} .156)$ with $1\left[\mathrm{Cd}\left(\mathrm{ClO}_{4}\right)_{2}\right.$. $\left.6 \mathrm{H}_{2} \mathrm{O}\right]$ per unit cell. West reported that the structure is very similar to the hexagonal magnesium perchlorate structure. Moss, Russell, and Sharp [2] confirmed West's determinations. The value of " $a$ " reported by West has been divided by 2 as he suggested. The unit cell values reported by West have been converted from $\mathrm{kX}$ to angstrom units for comparison with the NBS values.

\section{Lattice constants}

\begin{tabular}{|c|c|c|c|}
\hline & & $a$ & $c$ \\
\hline $\begin{array}{l}1935 \\
1963\end{array}$ & $\begin{array}{l}\text { West [1] } \\
\text { National Bureau of } \\
\text { Standards at } 25^{\circ} \mathrm{C} .\end{array}$ & $\begin{array}{c}\stackrel{\circ}{A} \\
\text { 7. } 92 \\
\text { 7. } 9939\end{array}$ & $\begin{array}{c}\stackrel{\AA}{A} \\
\text { 5. } 30 \\
\text { 5. } 3304\end{array}$ \\
\hline
\end{tabular}

The density of cadmium perchlorate hexahydrate calculated from the NBS lattice constants is $2.361 \mathrm{~g} / \mathrm{cm}^{3}$ at $25^{\circ} \mathrm{C}$.

\section{References}

[1] C. D. West, The crystal structures of hydrated compounds. II. Structure type $\mathrm{Mg}\left(\mathrm{ClO}_{4}\right)_{2} \cdot 6 \mathrm{H}_{2} \mathrm{O}, \mathrm{Z}$. Krist. A91, 480-493 (1935).

[2] K. C. Moss, D. R. Russell, and D. W. A. Sharp, The lattice constants of some metal fluoroborate hexahydrates, Acta Cryst. 14, 330 (1961).

\begin{tabular}{|c|c|c|}
\hline \multirow[t]{2}{*}{$h k l$} & \multicolumn{2}{|c|}{$\begin{array}{c}\text { Internal Standard, } \\
\text { Tungsten, } a=3.1648 \AA \\
\mathrm{Cu}, 1.5405 \AA \text { at } 25^{\circ} \mathrm{C}\end{array}$} \\
\hline & $d$ & $I$ \\
\hline $\begin{array}{l}100 \\
001 \\
101 \\
110 \\
200\end{array}$ & $\begin{array}{c}\AA \\
\text { 6. } 921 \\
\text { 5. } 326 \\
\text { 4. } 223 \\
\text { 3. } 995 \\
\text { 3. } 463\end{array}$ & $\begin{array}{r}25 \\
17 \\
75 \\
100 \\
4\end{array}$ \\
\hline $\begin{array}{l}111 \\
201 \\
002 \\
210 \\
102\end{array}$ & $\begin{array}{l}\text { 3. } 197 \\
\text { 2. } 902 \\
\text { 2. } 667 \\
\text { 2. } 617 \\
\text { 2. } 489\end{array}$ & $\begin{array}{r}51 \\
66 \\
2 \\
1 \\
18\end{array}$ \\
\hline $\begin{array}{l}211 \\
300 \\
112 \\
301 \\
202\end{array}$ & $\begin{array}{l}\text { 2. } 350 \\
\text { 2. } 309 \\
\text { 2. } 217 \\
\text { 2. } 117 \\
\text { 2. } 1119\end{array}$ & $\begin{array}{r}25 \\
9 \\
7 \\
26 \\
27\end{array}$ \\
\hline $\begin{array}{l}220 \\
310 \\
212 \\
311 \\
003\end{array}$ & $\begin{array}{l}\text { 1. } 9985 \\
\text { 1. } 9201 \\
\text { 1. } 8670 \\
\text { 1. } 8064 \\
\text { 1. } 7771\end{array}$ & $\begin{array}{r}19 \\
8 \\
37 \\
9 \\
3\end{array}$ \\
\hline $\begin{array}{l}302 \\
103 \\
401 \\
113 \\
320\end{array}$ & $\begin{array}{l}\text { 1. } 7446 \\
\text { 1. } 7205 \\
\text { 1. } 6463 \\
\text { 1. } 6231 \\
\text { 1. } 5880\end{array}$ & $\begin{array}{l}6 \\
7 \\
4 \\
3 \\
4\end{array}$ \\
\hline $\begin{array}{l}203 \\
312 \\
321 \\
410 \\
213\end{array}$ & $\begin{array}{l}\text { 1. } 5808 \\
\text { 1. } 5577 \\
\text { 1. } 5223 \\
\text { 1. } 5107 \\
\text { 1. } 4700\end{array}$ & $\begin{array}{l}9 \\
5 \\
6 \\
6 \\
5\end{array}$ \\
\hline $\begin{array}{l}411 \\
402 \\
303 \\
322 \\
501\end{array}$ & $\begin{array}{l}\text { 1. } 4539 \\
\text { 1. } 4519 \\
\text { 1. } 4078 \\
\text { 1. } 3642 \\
\text { 1. } 3402\end{array}$ & $\begin{array}{r}11 \\
11 \\
2 \\
3 \\
4\end{array}$ \\
\hline $\begin{array}{c}004,330 \\
420 \\
313 \\
421 \\
114\end{array}$ & $\begin{array}{l}\text { 1. } 3325 \\
\text { 1. } 3082 \\
\text { 1. } 3042 \\
\text { 1. } 2705 \\
\text { 1. } 2641\end{array}$ & $\begin{array}{l}4 \\
2 \\
5 \\
4 \\
4\end{array}$ \\
\hline $\begin{array}{l}204 \\
403 \\
502 \\
511 \\
214\end{array}$ & $\begin{array}{l}\text { 1. } 2438 \\
\text { 1. } 2400 \\
\text { 1. } 2287 \\
\text { 1. } 2107 \\
\text { 1. } 1872\end{array}$ & $\begin{array}{l}3 \\
3 \\
2 \\
5 \\
2\end{array}$ \\
\hline $\begin{array}{l}323 \\
422 \\
600 \\
413 \\
512\end{array}$ & $\begin{array}{l}\text { 1. } 1841 \\
\text { 1. } 1743 \\
\text { 1. } 1537 \\
\text { 1. } 1510 \\
\text { 1. } 1270\end{array}$ & $\begin{array}{l}3 \\
3 \\
3 \\
3 \\
3\end{array}$ \\
\hline 224 & 1. 1088 & 3 \\
\hline
\end{tabular}




\section{Cadmium Sulfate, $\mathrm{CdSO}_{4}$ (orthorhombic)}

Powder data cards

\begin{tabular}{|c|c|c|}
\hline $\begin{array}{c}\text { Card } \\
\text { number }\end{array}$ & $\begin{array}{c}\text { Index } \\
\text { lines }\end{array}$ & Source \\
\cline { 3 - 3 } $3-0453$ & $\begin{array}{r}3.30 \\
2.97 \\
2.35\end{array}$ & Dow Chemical company. \\
& & \\
\hline
\end{tabular}

Additional published patterns. None.

NBS sample. The sample of cadmium sulfate was prepared at NBS from cadmium carbonate treated with an excess of sulfuric acid and heated to dryness. Spectrographic analysis showed the major impurities to be 0.001 to 0.01 percent each of aluminum and silicon.

\begin{tabular}{|c|c|c|}
\hline \multirow[t]{2}{*}{$h k l$} & \multicolumn{2}{|c|}{$\begin{array}{l}\text { Internal Standard, } \\
\text { Tungsten, } a=3.1648 \AA \\
\mathrm{Cu}, 1.5405 \AA \text { at } 25^{\circ} \mathrm{C}\end{array}$} \\
\hline & $d$ & $I$ \\
\hline $\begin{array}{l}001 \\
110 \\
101 \\
020 \\
111\end{array}$ & $\begin{array}{c}\stackrel{\AA}{A} \\
\text { 4. } 701 \\
\text { 3. } 826 \\
\text { 3. } 329 \\
\text { 3. } 277 \\
\text { 2. } 961\end{array}$ & $\begin{array}{r}17 \\
41 \\
100 \\
79 \\
68\end{array}$ \\
\hline $\begin{array}{l}021 \\
200 \\
002 \\
121 \\
012\end{array}$ & $\begin{array}{l}\text { 2. } 688 \\
\text { 2. } 358 \\
\text { 2. } 352 \\
\text { 2. } 336 \\
2.212\end{array}$ & $\begin{array}{r}6 \\
35 \\
32 \\
37 \\
24\end{array}$ \\
\hline $\begin{array}{l}201 \\
211 \\
031 \\
220 \\
131\end{array}$ & $\begin{array}{l}\text { 2. } 1044 \\
\text { 2. } 0068 \\
\text { 1. } 9827 \\
\text { 1. } 9155 \\
\text { 1. } 8273\end{array}$ & $\begin{array}{l}26 \\
18 \\
22 \\
21 \\
17\end{array}$ \\
\hline $\begin{array}{l}122 \\
202 \\
040 \\
212 \\
032\end{array}$ & $\begin{array}{l}\text { 1. } 7704 \\
\text { 1. } 6648 \\
\text { 1. } 6391 \\
\text { 1. } 6135 \\
\text { 1. } 6004\end{array}$ & $\begin{array}{r}19 \\
3 \\
12 \\
18 \\
11\end{array}$ \\
\hline $\begin{array}{l}041 \\
310 \\
013 \\
231 \\
301\end{array}$ & $\begin{array}{l}\text { 1. } 5485 \\
\text { 1. } 5290 \\
\text { 1. } 5250 \\
\text { 1. } 5179 \\
1.4908\end{array}$ & $\begin{array}{l}3 \\
8 \\
7 \\
7 \\
6\end{array}$ \\
\hline $\begin{array}{l}222 \\
141 \\
311 \\
113 \\
321\end{array}$ & $\begin{array}{l}\text { 1. } 4843 \\
\text { 1. } 4704 \\
\text { 1. } 4537 \\
\text { 1. } 4497 \\
\text { 1. } 3571\end{array}$ & $\begin{array}{r}4 \\
9 \\
7 \\
17 \\
11\end{array}$ \\
\hline $\begin{array}{c}240 \\
232 \\
302,203 \\
142 \\
051,150\end{array}$ & $\begin{array}{l}\text { 1. } 3459 \\
\text { 1. } 3243 \\
\text { 1. } 3059 \\
\text { 1. } 2934 \\
\text { 1. } 2634\end{array}$ & $\begin{array}{l}6 \\
9 \\
4 \\
8 \\
1\end{array}$ \\
\hline $\begin{array}{l}331 \\
133 \\
151\end{array}$ & $\begin{array}{l}\text { 1. } 2317 \\
\text { 1. } 2293 \\
\text { 1. } 2204\end{array}$ & $\begin{array}{r}7 \\
11 \\
5\end{array}$ \\
\hline
\end{tabular}

The sample was white. The indices of refraction could not be determined because the sample was too fine.

The $d$-values of the three strongest lines are: $3.329,3.277$, and $2.961 \AA$.

Structural data. Kokkoros and Rentzeperis [1] in 1961 determined cadmium sulfate is orthorhombic with the most probable space group $\mathrm{D}_{2 \mathrm{~h}^{-}}^{13}$ Pmmn (No. 59) with 2(CdSO$\left.{ }_{4}\right)$ per unit cell.

Lattice constants

\begin{tabular}{|c|c|c|c|c|}
\hline & & $a$ & $b$ & $c$ \\
\hline 1961 & $\begin{array}{l}\text { Kokkoros and } \\
\text { Rentzeperis } \\
{[1] .}\end{array}$ & 4. $\stackrel{\stackrel{\circ}{7}}{709}$ & 6. $\stackrel{\AA}{\AA}$ & 4. $\stackrel{\AA}{A 94}$ \\
\hline 1963 & $\begin{array}{l}\text { National Bureau } \\
\text { of Standards } \\
\text { at } 25^{\circ} \mathrm{C} \text {. }\end{array}$ & 4. 7174 & 6. 5590 & 4. 7012 \\
\hline
\end{tabular}

The density of cadium sulfate calculated from NBS lattice constants is $4.759 \mathrm{~g} / \mathrm{cm}^{3}$ at $25^{\circ} \mathrm{C}$.

\begin{tabular}{|c|c|c|}
\hline \multirow[t]{2}{*}{$h k l$} & \multicolumn{2}{|c|}{$\begin{array}{l}\text { Internal Standard, } \\
\text { Tungsten, } a=3.1648 \AA \\
\mathrm{Cu}, 1.5405 \AA \text { at } 25^{\circ} \mathrm{C}\end{array}$} \\
\hline & $d$ & $I$ \\
\hline $\begin{array}{l}322 \\
223\end{array}$ & $\begin{array}{l}\stackrel{\circ}{A} \\
\text { 1. } 2140 \\
\text { 1. } 2127\end{array}$ & $\begin{array}{l}6 \\
4\end{array}$ \\
\hline $\begin{array}{l}400 \\
014 \\
052 \\
114 \\
251\end{array}$ & $\begin{array}{l}\text { 1. } 1794 \\
\text { 1. } 1570 \\
\text { 1. } 1454 \\
\text { 1. } 1235 \\
1.1136\end{array}$ & $\begin{array}{l}3 \\
3 \\
5 \\
3 \\
3\end{array}$ \\
\hline $\begin{array}{c}420,303 \\
024 \\
341 \\
313 \\
060\end{array}$ & $\begin{array}{l}\text { 1. } 1100 \\
\text { 1. } 1062 \\
\text { 1. } 1032 \\
\text { 1. } 0943 \\
\text { 1. } 0933\end{array}$ & $\begin{array}{l}3 \\
5 \\
4 \\
6 \\
5\end{array}$ \\
\hline $\begin{array}{l}421 \\
124 \\
204 \\
412 \\
161\end{array}$ & $\begin{array}{l}\text { 1. } 0798 \\
\text { 1. } 0777 \\
\text { 1. } 0519 \\
\text { 1. } 0407 \\
\text { 1. } 0387\end{array}$ & $\begin{array}{l}2 \\
2 \\
4 \\
7 \\
6\end{array}$ \\
\hline $\begin{array}{l}252 \\
342 \\
134 \\
224 \\
260\end{array}$ & $\begin{array}{l}\text { 1. } 0305 \\
\text { 1. } 0221 \\
\text { 1. } 0113 \\
\text { 1. } 0016 \\
\text { 0. } 9918\end{array}$ & $\begin{array}{l}3 \\
2 \\
3 \\
3 \\
3\end{array}$ \\
\hline $\begin{array}{c}333 \\
351,153 \\
261 \\
440 \\
044 \\
432\end{array}$ & $\begin{array}{l}.9897 \\
.9839 \\
.9702 \\
.9576 \\
.9552 \\
.9498\end{array}$ & $\begin{array}{l}5 \\
4 \\
3 \\
3 \\
4 \\
5\end{array}$ \\
\hline
\end{tabular}

\section{Reference}

[1] P. A. Kokkoros and P. J. Rentzeperis, X-ray investigation of the anhydrous cadmium and mercuric sulphates, Acta Cryst. 14, 329-330 (1961). 


\section{Cadmium Telluride, CdTe (cubic)}

\section{Powder data cards}

\begin{tabular}{|c|c|c|}
\hline $\begin{array}{c}\text { Card } \\
\text { Number }\end{array}$ & $\begin{array}{c}\text { Index } \\
\text { Lines }\end{array}$ & Souree \\
\hline $10-207$ & $\begin{array}{r}3.74 \\
2.29\end{array}$ & Vaughan [1]. \\
& $\begin{array}{r}1.95 \\
\hline\end{array}$ \\
\hline
\end{tabular}

Additional published patterns. None.

NBS sample. The sample of cadmium telluride was obtained from Semi-Elements Inc., Saxonburg, Pa. Spectrographic analysis showed the following impurities: 0.01 to 0.1 percent silicon and 0.0001 to 0.001 percent each of barium, iron, and lead.

The sample was a black opaque powder.

The $d$-values of the three strongest lines are: $3.741,2.290$, and $1.954 \AA$.

Structural data. Zachariasen [2] in 1926 determined that cadmium telluride has the zinc sulfide structure, the space group $\mathrm{T}_{\mathrm{d}}^{2}-\mathrm{F} \overline{4} 3 \mathrm{~m}$ (No. 216), and $4(\mathrm{CdTe})$ per unit cell. The lattice constants of Zachariasen and Goldschmidt have been converted from $\mathrm{kX}$ to angstrom units for comparison with the NBS value.

\section{Lattice constant}

\begin{tabular}{|c|c|c|}
\hline $\begin{array}{l}1926 \\
1926 \\
1963\end{array}$ & $\begin{array}{l}\text { Zachariasen }[2] \\
\text { Goldsehmidt }[3] \\
\text { National Bureau of Standards } \\
\quad \text { at } 25^{\circ} \mathrm{C}\end{array}$ & $\begin{array}{c}\stackrel{\AA}{A} \\
\text { 6. } 477 \\
\text { 6. } 453 \\
\text { 6. } 481\end{array}$ \\
\hline
\end{tabular}

The density of cadium telluride calculated from the NBS lattice constant is $5.856 \mathrm{~g} / \mathrm{cm}^{3}$ at $25^{\circ} \mathrm{C}$.

\begin{tabular}{|c|c|c|c|}
\hline \multirow[t]{2}{*}{$h k l$} & \multicolumn{3}{|c|}{$\begin{array}{l}\text { Internal Standard, } \\
\text { Tungsten, } a=3.1648 \AA \\
\mathrm{Cu}, 1.5405 \AA \text { at } 25^{\circ} \mathrm{C}\end{array}$} \\
\hline & $d$ & $I$ & $a$ \\
\hline $\begin{array}{l}111 \\
220 \\
311 \\
400 \\
331\end{array}$ & $\begin{array}{l}\stackrel{\AA}{A} \\
3.742 \\
2.290 \\
1.954 \\
1.619 \\
1.488\end{array}$ & $\begin{array}{r}100 \\
62 \\
28 \\
5 \\
9\end{array}$ & $\begin{array}{l}\AA \\
6.481 \\
6.479 \\
6.482 \\
6.476 \\
6.484\end{array}$ \\
\hline $\begin{array}{l}422 \\
511 \\
440 \\
531 \\
620\end{array}$ & $\begin{array}{l}1.323 \\
1.247 \\
1.146 \\
1.095 \\
1.025\end{array}$ & $\begin{array}{r}10 \\
4 \\
2 \\
4 \\
4\end{array}$ & $\begin{array}{l}\text { 6. } 482 \\
\text { 6. } 481 \\
\text { 6. } 480 \\
\text { 6. } 479 \\
6.481\end{array}$ \\
\hline $\begin{array}{l}533 \\
444 \\
711 \\
642 \\
731\end{array}$ & $\begin{array}{r}0.9884 \\
.9356 \\
.9076 \\
.8661 \\
.8438\end{array}$ & $\begin{array}{r}2 \\
<1 \\
2 \\
3 \\
3\end{array}$ & $\begin{array}{l}\text { 6. } 481 \\
\text { 6. } 482 \\
\text { 6. } 481 \\
\text { 6. } 481 \\
6.482\end{array}$ \\
\hline & of las & & 6. 481 \\
\hline
\end{tabular}

\section{References}

[1] D. A. Vaughan, Battelle Memorial Institute, Columbus, Ohio.

[2] W. H. Zachariasen, Úber die Kristallstruktur der Telluride von Beryllium, Zink, Cadmium, und Quecksilver, Z. physik Chem. 124, 277 (1926).

[3] V. M. Goldschmidt, Geoehemisehe Verteilungsgesetze der Elements VII Die Gesetze der Krystalloehemie, Skrifter Norske Videnskaps-Adad. Oslo, I: Mat.Naturv. Kl. No. 2 (1926). 
Calcium Fluoride Phosphate (fluorapatite), $\mathbf{C a} \mathbf{a}_{5} \mathbf{F}\left(\mathbf{P O}_{4}\right)_{3}$ (hexagonal)

Powder data cards

\begin{tabular}{|c|c|c|}
\hline $\begin{array}{l}\text { Card } \\
\text { number }\end{array}$ & $\begin{array}{l}\text { Index } \\
\text { lines }\end{array}$ & Source \\
\hline $3-0736$ & $\begin{array}{l}\text { 2. } 81 \\
\text { 2. } 71 \\
\text { 1. } 84\end{array}$ & McConnell [4] 1937. \\
\hline $12-261$ & $\begin{array}{l}2.82 \\
2.72 \\
2.26\end{array}$ & Carobbi and Mazzi [13] 1959. \\
\hline
\end{tabular}

\begin{tabular}{|c|c|c|}
\hline \multirow[t]{2}{*}{$h k l$} & \multicolumn{2}{|c|}{$\begin{array}{l}\text { Internal Standard, } \\
\text { Tungsten, } a=3.1648 \AA \\
\mathrm{Cu}, 1.5405 \AA \text { at } 25^{\circ} \mathrm{C}\end{array}$} \\
\hline & $d$ & $I$ \\
\hline $\begin{array}{l}100 \\
101 \\
110 \\
200 \\
111\end{array}$ & $\begin{array}{ll} & \AA \\
\text { 8. } & 12 \\
\text { 5. } 25 \\
\text { 4. } 684 \\
\text { 4. } 055 \\
\text { 3. } 872\end{array}$ & $\begin{array}{r}8 \\
4 \\
<1 \\
8 \\
8\end{array}$ \\
\hline $\begin{array}{l}201 \\
002 \\
102 \\
210 \\
211\end{array}$ & $\begin{array}{l}\text { 3. } 494 \\
\text { 3. } 442 \\
\text { 3. } 167 \\
\text { 3. } 067 \\
\text { 2. } 800\end{array}$ & $\begin{array}{r}<1 \\
42 \\
13 \\
17 \\
100\end{array}$ \\
\hline $\begin{array}{l}112 \\
300 \\
202 \\
301 \\
212\end{array}$ & $\begin{array}{l}\text { 2. } 772 \\
\text { 2. } 702 \\
\text { 2. } 624 \\
\text { 2. } 517 \\
\text { 2. } 289\end{array}$ & $\begin{array}{r}54 \\
62 \\
29 \\
6 \\
7\end{array}$ \\
\hline $\begin{array}{l}310 \\
221 \\
311 \\
302 \\
113\end{array}$ & $\begin{array}{l}\text { 2. } 250 \\
\text { 2. } 218 \\
\text { 2. } 140 \\
\text { 2. } 128 \\
\text { 2. } 061\end{array}$ & $\begin{array}{r}22 \\
3 \\
6 \\
3 \\
5\end{array}$ \\
\hline $\begin{array}{l}400 \\
203 \\
222 \\
312 \\
320\end{array}$ & $\begin{array}{l}\text { 2. } 028 \\
\text { 1. } 997 \\
\text { 1. } 937 \\
\text { 1. } 884 \\
\text { 1. } 862\end{array}$ & $\begin{array}{r}1 \\
4 \\
26 \\
14 \\
4\end{array}$ \\
\hline $\begin{array}{l}213 \\
321 \\
410 \\
402 \\
004\end{array}$ & $\begin{array}{l}\text { 1. } 837 \\
\text { 1. } 797 \\
\text { 1. } 771 \\
\text { 1. } 748 \\
\text { 1. } 722\end{array}$ & $\begin{array}{l}32 \\
15 \\
13 \\
13 \\
15\end{array}$ \\
\hline $\begin{array}{l}104 \\
322 \\
313 \\
501 \\
330\end{array}$ & $\begin{array}{l}\text { 1. } 684 \\
\text { 1. } 637 \\
\text { 1. } 607 \\
\text { 1. } 580 \\
\text { 1. } 562\end{array}$ & $\begin{array}{r}<1 \\
6 \\
3 \\
1 \\
<1\end{array}$ \\
\hline $\begin{array}{l}420 \\
331 \\
214 \\
421 \\
502\end{array}$ & $\begin{array}{l}1.534 \\
\text { 1. } 524 \\
\text { 1. } 501 \\
1.497 \\
1.468\end{array}$ & $\begin{array}{l}5 \\
4 \\
4 \\
4 \\
8\end{array}$ \\
\hline $\begin{array}{l}510 \\
304 \\
323\end{array}$ & $\begin{array}{l}\text { 1. } 457 \\
\text { 1. } 452 \\
\text { 1. } 446\end{array}$ & $\begin{array}{l}4 \\
7 \\
6\end{array}$ \\
\hline
\end{tabular}

Additional published patterns. Náray-Szabó [2] 1930, Bale [6] 1940.

NBS sample. The sample of calcium fluoride phosphate was prepared at NBS by J. H. deGroot. A mixture of calcium fluoride and tricalcium orthophosphate was heated in a covered platinum dish to $1250^{\circ} \mathrm{C}$. Spectrographic analysis showed the following major impurities: 0.1 to 1.0 percent each of magnesium and sodium; and 0.01 to 0.1 percent each of aluminum, barium, germanium, iron, silicon, and strontium.

\begin{tabular}{|c|c|c|}
\hline \multirow[t]{2}{*}{$h k l$} & \multicolumn{2}{|c|}{$\begin{array}{l}\text { Internal Standard, } \\
\text { Tungsten, } a=3.1648 \AA \\
\mathrm{Cu}, 1.5405 \AA \text { at } 25^{\circ} \mathrm{C}\end{array}$} \\
\hline & $d$ & $I$ \\
\hline $\begin{array}{l}511 \\
332\end{array}$ & $\begin{array}{l}\stackrel{\circ}{A} \\
\text { 1. } 426 \\
\text { 1. } 422\end{array}$ & $\begin{array}{l}5 \\
4\end{array}$ \\
\hline $\begin{array}{l}413 \\
512 \\
430 \\
431 \\
520\end{array}$ & $\begin{array}{l}\text { 1. } 4015 \\
\text { 1. } 3418 \\
\text { 1. } 3336 \\
\text { 1. } 3095 \\
1.2995\end{array}$ & $\begin{array}{r}2 \\
2 \\
<1 \\
3 \\
2\end{array}$ \\
\hline $\begin{array}{l}521 \\
423 \\
324 \\
215 \\
432\end{array}$ & $\begin{array}{l}\text { 1. } 2763 \\
\text { 1. } 2748 \\
\text { 1. } 2635 \\
\text { 1. } 2560 \\
\text { 1. } 2438\end{array}$ & $\begin{array}{r}3 \\
2 \\
<1 \\
3 \\
2\end{array}$ \\
\hline $\begin{array}{l}610 \\
414 \\
305 \\
611 \\
522\end{array}$ & $\begin{array}{l}\text { 1. } 2372 \\
\text { 1. } 2341 \\
\text { 1. } 2271 \\
\text { 1. } 2176 \\
\text { 1. } 2154\end{array}$ & $\begin{array}{l}4 \\
6 \\
9 \\
4 \\
3\end{array}$ \\
\hline $\begin{array}{l}504 \\
315 \\
440 \\
334 \\
433\end{array}$ & $\begin{array}{l}\text { 1. } 1805 \\
\text { 1. } 1743 \\
\text { 1. } 1709 \\
\text { 1. } 1563 \\
\text { 1. } 1532\end{array}$ & $\begin{array}{r}<1 \\
<1 \\
1 \\
2 \\
3\end{array}$ \\
\hline $\begin{array}{l}006 \\
424 \\
106 \\
523 \\
116\end{array}$ & $\begin{array}{l}\text { 1. } 1474 \\
\text { 1. } 1448 \\
\text { 1. } 1359 \\
\text { 1. } 1306 \\
\text { 1. } 1144\end{array}$ & $\begin{array}{r}1 \\
2 \\
<1 \\
<1 \\
3\end{array}$ \\
\hline $\begin{array}{c}514 \\
325 \\
702,532 \\
710,216 \\
622\end{array}$ & $\begin{array}{l}\text { 1. } 1118 \\
\text { 1. } 1069 \\
\text { 1. } 0984 \\
\text { 1. } 0745 \\
\text { 1. } 0694\end{array}$ & $\begin{array}{r}2 \\
2 \\
5 \\
<1 \\
<1\end{array}$ \\
\hline $\begin{array}{c}524 \\
703,533 \\
335 \\
226 \\
712\end{array}$ & $\begin{array}{l}\text { 1. } 0368 \\
\text { 1. } 0346 \\
\text { 1. } 0326 \\
\text { 1. } 0303 \\
\text { 1. } 0257\end{array}$ & $\begin{array}{r}<1 \\
<1 \\
2 \\
2 \\
2\end{array}$ \\
\hline $\begin{array}{c}630,316 \\
800 \\
631 \\
614 \\
406\end{array}$ & $\begin{array}{l}\text { 1. } 0221 \\
1.0140 \\
1.0110 \\
1.0047 \\
0.9986\end{array}$ & $\begin{array}{r}2 \\
1 \\
1 \\
1 \\
<1\end{array}$ \\
\hline
\end{tabular}




\section{Calcium Fluoride Phosphate (fluorapatite), $\mathrm{Ca}_{5} \mathbf{F}\left(\mathbf{P O}_{4}\right)_{3}$ (hexagonal)-Continued}

The sample was colorless and optically negative with the indices of refraction $\mathrm{N}_{0}=1.633$ and $\mathrm{N}_{\mathrm{e}}$ $=1.628$.

The $d$-values of the three strongest lines are: $2.800,2.702$, and $2.772 \AA$.
Structural data. Hentschel [1] in 1923 determined that fluorapatite has the space group $\mathrm{C}_{6 \mathrm{n}}^{2}-\mathrm{P} 6 / \mathrm{m}$ (No. 176) and $2\left[\mathrm{Ca}_{5} \mathrm{~F}\left(\mathrm{PO}_{4}\right)_{3}\right]$ per unit cell.

The density of synthetic fluorapatite calculated from the NBS lattice constants is $3.201 \mathrm{~g} / \mathrm{cm}^{3}$ at $25{ }^{\circ} \mathrm{C}$.

Lattice constants

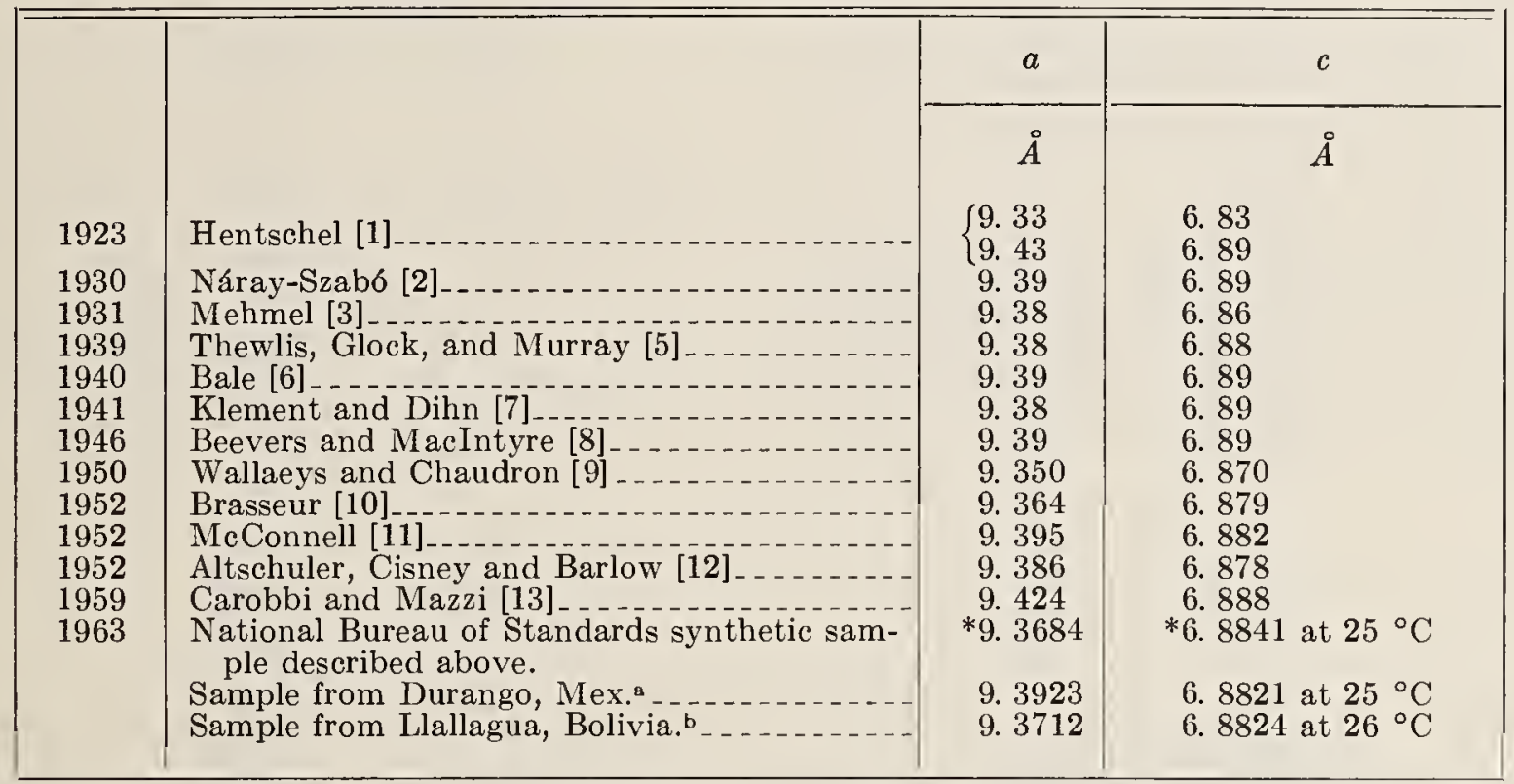

*The error in these values was \pm 0.0003

a.b These samples were obtained from the National Museum; the number of the Mexican sample is 104021, of the Bolivian sample 103869 . These cell constants were derived from powder diffraction measurements made at the same time as those on the synthetie sample.

a This particular sample from Durango may not be typical because of its lanthanum content. Spectrographic analysis showed 1 to 4 percent of lanthanu in nd 0.1 to 1.0 percent each of cerium and sodium as major impurities. The error on these lattice constants was \pm 0.0005 .

b Spectrographic analysis showed major impurities to be 0.1 to 1.0 percent of manganese and 0.01 to 0.1 percent each of iron and strontium.

\section{References}

[1] H. Hentschel, Röntgenographische Untersuchungen am Apatit, Centr. Mineral. Geol. p. 609 (1923).

[2] St. Náray-Szabó, The structure of apatite $(\mathrm{CaF}) \mathrm{Ca}_{4}$ $\left(\mathrm{PO}_{4}\right)_{3}$, Z. Krist. 75, 387-398 (1930).

[3] M. Mehmel, Beziehungen zwischen kristallstruktur und chemischer formel des apatits, Z. physik Chem. B15, 223-41 (1931).

[4] D. MeConnell, The substitution of $\mathrm{SiO}_{4}-$ and $\mathrm{SO}_{4-}$ groups for $\mathrm{PO}_{4}$-groups in the apatite structure; ellestadite, the end-member, Am. Mineralogist 22, 981 (1937).

[5] J. Thewlis, G. E. Glock, and M. M. Murray, Chemical and $x-$ Ray analysis of dental, mineral, and synthetio apatites, Trans. Faraday Soc. 35, 358-63 (1939).

[6] W. F. Bale, A comparative roentgen-ray diffraction study of several natural apatites and the apatitelike constituent of bone and tooth substance, Am. J. Roentgenol. 43, 735-47 (1940).

[7] R. Illement and P. Dihn, Isomorphe Apatitarten, Naturwissenschaften 29, 301 (1941).
[8] C. A. Beevers and D. B. Macintyre, The atomic structure of fluorapatite and its relation to that of tooth and bone material, Mineralog. Mag. 27, 254 (1946).

[9] R. Wallaeys and G. Chaudron, Sur la preparation de certaines apatites mixtes, Compt. lend. 231, 355357 (1950).

[10] H. Brasseur, Note sur les constantes reticulaires et les indices de refraction des fluor-, chlor-, et hydroxylapatites, Proceedings of the International Symposium on the reactivity of solids, Gothenburg Part 1, 363-7 (1952).

[11] D. MeConnell, The Problem of the carbonate apatites IV, Structural substitutions involving $\mathrm{CO}_{3}$ and $\mathrm{OH}$, Bull. Soc. Frane. Mineral. et Crist. 95, 428 (1952).

[12] Z. S. Altschuler, E. A. Cisney, and I. H. Barlow, X-Ray evidence of the nature of earbonate-apatite, Bull. Geol. Soc. Amer. 63, 1230-31 (1952).

[13] G. Carobbi and F. Mazri, Sulla possibilita di una sostituzione parziale del calcio eon l'uranio nel reticolo dell' apatite, Atti. acead. 11a\%. Lincei, Mem. Classe Sei. Fis. Mat. Nat. Ser. IIa [8] 5, 159-71 (1959). 


\section{Cerium Niobium Titanium Oxide (eschynite), $\mathrm{CeNbTiO}_{6}$ (orthorhombic)}

Powder data cards. None.

Additional published patterns. Komkov [1] 1959.

NBS sample. The sample of cerium niobium titanium oxide was prepared at NBS by $R$. S. Roth from stoichiometric mixtures of cerium oxide, niobium oxide, and titanium oxide. The sample was heated first at $1300^{\circ} \mathrm{C}$ for $3 \mathrm{hrs}$. and then reheated at $1325{ }^{\circ} \mathrm{C}$ for three more hours. Spectrographic analysis showed the following major impurities: 0.001 to 0.01 percent each of aluminum, calcium, iron, magnesium, and silicon.

The sample was a dark brown opaque powder.

The $d$-values of the three strongest lines are: $2.975,3.024$, and $3.106 \AA$.

Structural data. Komkov [1] in 1959 determined that cerium niobium titanium oxide has the space group $\mathrm{D}_{2 \mathrm{~h}}^{16}-\mathrm{Pmnb}\left(\mathrm{No}_{0} 62\right)$ with $4\left(\mathrm{CeNbTiO}_{6}\right)$ per unit cell. The lattice constants reported by Komkov have been converted from $\mathrm{kX}$ to angstrom units for comparison with NBS values.

Lattice constants

\begin{tabular}{|c|c|c|c|c|}
\hline & & $a$ & $b$ & $c$ \\
\hline 1959 & Komkov $[1]$ & 7. $\stackrel{\AA}{56}$ & $\begin{array}{c}\stackrel{\circ}{A} \\
10.99\end{array}$ & 5. $\stackrel{\AA}{4}$ \\
\hline 1963 & $\begin{array}{l}\text { National Bureau of } \\
\text { Standards at } 26^{\circ} \mathrm{C} \text {. }\end{array}$ & 7. 538 & 10. 958 & 5. 396 \\
\hline
\end{tabular}

The density of cerium niobium titanium oxide calculated from NBS lattice constants is 5.617 $\mathrm{g} / \mathrm{cm}^{3}$ at $26^{\circ} \mathrm{C}$.

\section{Reference}

[1] A. I. Komkov, Minerals of the series euxenite-polycrase and priorite-blomstrandite, Dokl. Akad. Nauk SSSR 126, 641-644 (1959).

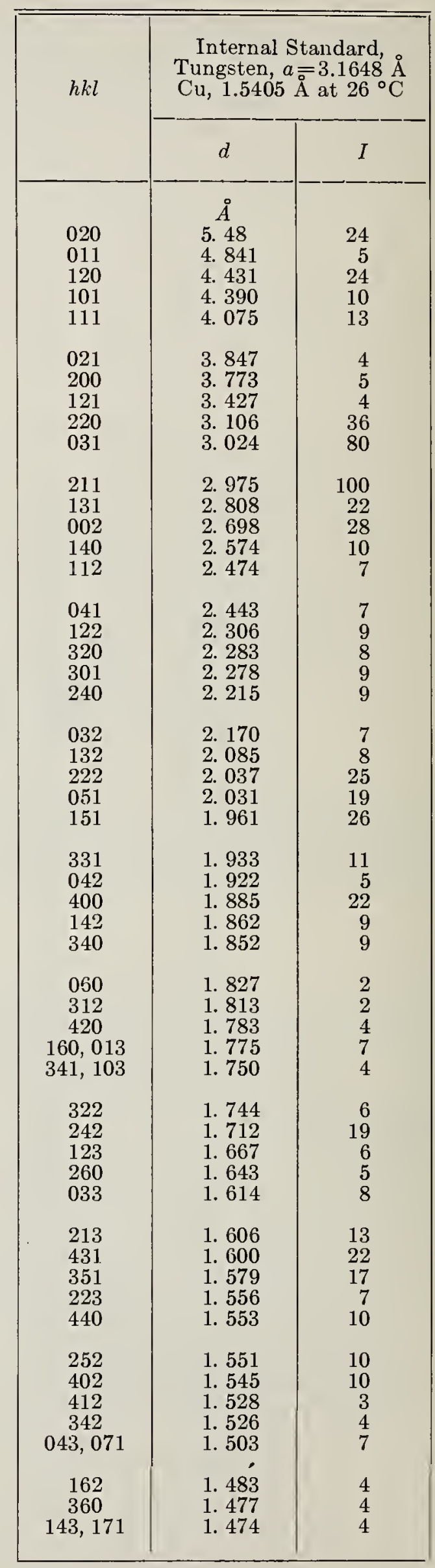




\section{Cesium Chromate, $\mathrm{Cs}_{2} \mathrm{CrO}_{4}$ (orthorhombic)}

Powder data cards. None.

Additional published patterns. None.

NBS sample. The sample of cesium chromate was obtained from the Fairmount Chemical Co. Inc., Newark, N.J. Spectrographic analysis showed the following major impurities: 0.1 to 1.0 percent rubidium; 0.01 to 0.1 percent each of potassium and sodium; and 0.001 to 0.01 percent each of aluminum, barium, calcium, and silicon.

The color of the sample was yellow and it is optically positive. The refractive indices are $\mathrm{N}_{\alpha}=1.750, \mathrm{~N}_{\beta}=1.753$, and $\mathrm{N}_{\gamma}=1.762$.

The $d$-values of the three strongest lines are: $3.342,3.207$, and $3.364 \AA$.

Structural data. Miller [1] in 1938 determined that cesium chromate has the potassium sulfate structure, the space group $D_{2 \mathrm{~h}}^{10}-$ Pnam (No. 62) with $4\left(\mathrm{Cs}_{2} \mathrm{CrO}_{4}\right)$ per unit cell.

The lattice constants reported by Miller have been converted from $\mathrm{kX}$ to angstrom units for comparison with NBS values.

\section{Lattice constants}

\begin{tabular}{|c|c|c|c|c|}
\hline \multirow{3}{*}{$\begin{array}{l}1938 \\
1963\end{array}$} & \multirow{3}{*}{$\begin{array}{l}\text { Miller }[1] \\
\text { National Bureau } \\
\text { of Standards } \\
25^{\circ} \mathrm{C}\end{array}$} & $a$ & $b$ & $c$ \\
\hline & & $\begin{array}{c}\stackrel{\circ}{A} \\
\text { 8. } 380\end{array}$ & $\begin{array}{c}\stackrel{\AA}{A} \\
11.157\end{array}$ & $\begin{array}{c}\stackrel{\circ}{A} \\
\text { 6. } 239\end{array}$ \\
\hline & & $\begin{array}{r}8.429 \\
\pm 0.001\end{array}$ & $\begin{array}{r}11.190 \\
\pm 0.001\end{array}$ & $\begin{array}{r}6.302 \\
\pm 0.001\end{array}$ \\
\hline
\end{tabular}

The density of cesium chromate calculated from the NBS lattice constants is $4.266 \mathrm{~g} / \mathrm{cm}^{3}$ at $25{ }^{\circ} \mathrm{C}$.

\section{Reference}

[1] J. J. Miller, The structure of $\mathrm{Cs}_{2} \mathrm{CrO}_{4}, \mathrm{Z}$. Krist. 99A, 32-37 (1938).

\begin{tabular}{|c|c|c|}
\hline \multirow[t]{2}{*}{$h k l$} & \multicolumn{2}{|c|}{$\begin{array}{c}\text { Internal Standard, } \\
\text { Tungsten, } a=3.1648 \AA \\
\mathrm{Cu}, 1.5405 \AA \text { at } 25{ }^{\circ} \mathrm{C}\end{array}$} \\
\hline & $d$ & $I$ \\
\hline $\begin{array}{l}120 \\
111 \\
200 \\
210 \\
121\end{array}$ & $\begin{array}{l}\quad \stackrel{\AA}{A} \\
4.66 \\
4.602 \\
\text { 4. } 213 \\
\text { 3. } 946 \\
\text { 3. } 749\end{array}$ & $\begin{array}{r}8 \\
9 \\
8 \\
20 \\
69\end{array}$ \\
\hline $\begin{array}{l}201 \\
130 \\
220 \\
211 \\
031\end{array}$ & $\begin{array}{l}3.505 \\
3.411 \\
3.364 \\
3.342 \\
3.207\end{array}$ & $\begin{array}{r}18 \\
21 \\
77 \\
100 \\
87\end{array}$ \\
\hline $\begin{array}{l}002 \\
131 \\
221 \\
230 \\
310\end{array}$ & $\begin{array}{l}\text { 3. } 150 \\
\text { 2. } 998 \\
\text { 2. } 969 \\
2.794 \\
2.725\end{array}$ & $\begin{array}{r}66 \\
8 \\
17 \\
24 \\
34\end{array}$ \\
\hline $\begin{array}{l}140 \\
122 \\
231 \\
311 \\
212\end{array}$ & $\begin{array}{l}\text { 2. } 654 \\
\text { 2. } 611 \\
\text { 2. } 554 \\
\text { 2. } 501 \\
\text { 2. } 463\end{array}$ & $\begin{array}{r}11 \\
15 \\
6 \\
6 \\
17\end{array}$ \\
\hline $\begin{array}{l}141 \\
321,240 \\
132 \\
222 \\
330\end{array}$ & $\begin{array}{l}\text { 2. } 449 \\
\text { 2. } 331 \\
\text { 2. } 314 \\
\text { 2. } 301 \\
\text { 2. } 244\end{array}$ & $\begin{array}{l}17 \\
13 \\
31 \\
29 \\
13\end{array}$ \\
\hline $\begin{array}{l}241 \\
150 \\
051,400 \\
232 \\
312\end{array}$ & $\begin{array}{l}\text { 2. } 186 \\
\text { 2. } 163 \\
\text { 2. } 109 \\
\text { 2. } 090 \\
\text { 2. } 062\end{array}$ & $\begin{array}{r}8 \\
15 \\
10 \\
15 \\
15\end{array}$ \\
\hline $\begin{array}{l}113 \\
401 \\
340 \\
250 \\
420\end{array}$ & $\begin{array}{l}2.004 \\
1.999 \\
1.982 \\
1.977 \\
1.971\end{array}$ & $\begin{array}{r}10 \\
9 \\
7 \\
10 \\
14\end{array}$ \\
\hline $\begin{array}{l}411 \\
123 \\
341 \\
242 \\
060\end{array}$ & $\begin{array}{l}\text { 1. } 967 \\
1.9152 \\
1.8911 \\
1.8743 \\
1.8653\end{array}$ & $\begin{array}{r}8 \\
8 \\
26 \\
7 \\
6\end{array}$ \\
\hline $\begin{array}{l}213 \\
430 \\
033 \\
332 \\
152\end{array}$ & $\begin{array}{l}1.8542 \\
1.8349 \\
1.8308 \\
1.8280 \\
1.7833\end{array}$ & $\begin{array}{r}13 \\
6 \\
19 \\
8 \\
16\end{array}$ \\
\hline $\begin{array}{l}431 \\
402 \\
161 \\
260 \\
143\end{array}$ & $\begin{array}{l}\text { 1. } 7615 \\
1.7521 \\
1.7499 \\
1.7060 \\
1.6474\end{array}$ & $\begin{array}{r}4 \\
11 \\
6 \\
4 \\
7\end{array}$ \\
\hline 441 & 1. 6263 & 6 \\
\hline
\end{tabular}




\section{Cesium Fluoride, CsF (cubic)}

\section{Powder data cards}

\begin{tabular}{|r|r|l|}
\hline $\begin{array}{c}\text { Card } \\
\text { number }\end{array}$ & $\begin{array}{c}\text { Index } \\
\text { lines }\end{array}$ & Source \\
\hline $1-0872$ & $\begin{array}{r}3.00 \\
2.12 \\
3.48\end{array}$ & Davey [1] 1923. \\
\hline
\end{tabular}

Additional published patterns. None.

NBS sample. The sample of cesium fluoride was obtained from Semi-Elements Inc., Saxonburg, $\mathrm{Pa}$. X-ray patterns were prepared from samples maintained in dry air because of the deliquescense. Spectrographic analysis showed the following impurities: 0.1 to 1.0 percent potassium; and 0.001 to 0.01 percent each of aluminum, calcium, copper, iron, lithium, magnesium, nickel, silicon, and sodium. The amaunt of rubidium impurity was not determined. The sample was colorless.

The $d$-values of the three strongest lines are: $3.003,3.469$, and $2.125 \AA$.

Structural data. Posnjak and Wyckoff [2] in 1922 determined that cesium fluoride has the sodium chloride structure, the space group $\mathrm{O}_{\mathrm{h}}^{5}$ Fm3m (No. 225), and 4(CsF) per unit cell. The unit cell measurements reported by Davey, Posnjak, and Wyckoff, have been converted from $\mathrm{kX}$ to angstrom units for comparison with the NBS value.

\section{Lattice constants}

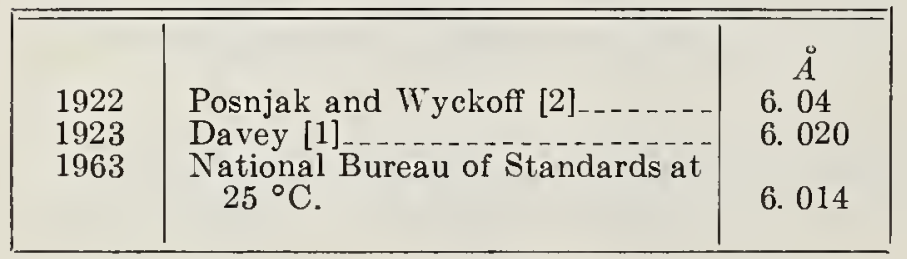

The density of cesium fluoride calculated from the NBS lattice constant is $4.638 \mathrm{~g} / \mathrm{cm}^{3}$ at $25^{\circ} \mathrm{C}$.

\begin{tabular}{|c|c|c|c|}
\hline \multirow[t]{2}{*}{$h k l$} & \multicolumn{3}{|c|}{$\begin{array}{l}\text { Internal Standard, } \\
\text { Tungsten, } a=3.1648 \AA \\
\mathrm{Cu}, 1.5405 \AA \text { at } 25^{\circ} \mathrm{C}\end{array}$} \\
\hline & $d$ & $I$ & $a$ \\
\hline $\begin{array}{l}111 \\
200 \\
220 \\
311 \\
222\end{array}$ & $\begin{array}{l}\quad \AA \\
\text { 3. } 469 \\
\text { 3. } 003 \\
\text { 2. } 125 \\
\text { 1. } 8131 \\
\text { 1. } 7366\end{array}$ & $\begin{array}{r}81 \\
100 \\
37 \\
25 \\
10\end{array}$ & $\begin{array}{c}\stackrel{\AA}{A} \\
6.008 \\
6.007 \\
6.011 \\
6.013 \\
6.016\end{array}$ \\
\hline $\begin{array}{l}400 \\
331 \\
420 \\
422\end{array}$ & $\begin{array}{l}\text { 1. } 5036 \\
\text { 1. } 3798 \\
\text { 1. } 3446 \\
1.2278\end{array}$ & $\begin{array}{r}7 \\
6 \\
10 \\
5\end{array}$ & $\begin{array}{l}6.014 \\
6.014 \\
6.013 \\
6.015\end{array}$ \\
\hline $\begin{array}{l}511 \\
440 \\
531 \\
600 \\
620\end{array}$ & $\begin{array}{l}1.1576 \\
1.0630 \\
1.0166 \\
1.0024 \\
0.9512\end{array}$ & $\begin{array}{l}4 \\
2 \\
3 \\
3 \\
4\end{array}$ & $\begin{array}{l}\text { 6. } 015 \\
6.013 \\
6.014 \\
6.014 \\
6.016\end{array}$ \\
\hline $\begin{array}{l}533 \\
622 \\
711 \\
642 \\
731\end{array}$ & $\begin{array}{l}.9173 \\
.9067 \\
.8420 \\
.8036 \\
.7830\end{array}$ & $\begin{array}{l}1 \\
2 \\
2 \\
1 \\
2\end{array}$ & $\begin{array}{l}\text { 6. } 015 \\
6.014 \\
6.013 \\
6.014 \\
6.014\end{array}$ \\
\hline \multicolumn{3}{|c|}{ Average value of last five lines } & 6.014 \\
\hline
\end{tabular}

\section{References}

[1] W. P. Davey, Precision measurements of crystals of the alkali halides, Phys. Rev. 21, 143-61 (1923).

[2] E. Posnjak and R. W. G. Wyckoff, The crystal structures of the alkali halides II, J. Wash. Acad. Sci. 12, 248-51 (1922). 


\section{Cobalt Fluosilicate Hexahydrate, $\mathrm{CoSiF}_{6} \cdot 6 \mathrm{H}_{2} \mathrm{O}$ (trigonal)}

Powder Data cards. None.

Additional published patterns. None.

NBS sample. The sample of cobalt fluosilicate hexahydrate was obtained from the City Chemical Co., New York, N.Y. Spectrographic analysis showed the following major impurities: 0.01 to 0.1 percent each of aluminum, copper, magnesium, nickel, and sodium; and 0.001 to 0.01 percent each of calcium, iron, and manganese.

The color of the sample was pink. The indices of refraction were too low to be measured by the usual liquid grain immersion method.

The $d$-values of the three strongest lines are: $4.69,4.18$, and $2.595 \AA$.

Structural data. Hassel and Richter-Salvesen [1] in 1927 determined that cobalt fluosilicate bexahydrate has the nickel chlorostannate structure, the space group $\mathrm{C}_{3 \mathrm{v}}^{5}-\mathrm{R} 3 \mathrm{~m}$ (No. 160), and $1\left[\mathrm{CoSiF}_{6} \cdot 6 \mathrm{H}_{2} \mathrm{O}\right]$ per unit rhombohedral cell, or $3\left[\mathrm{CoSiF}_{6} \cdot 6 \mathrm{H}_{2} \mathrm{O}\right]$ per unit hexagonal cell. The unit cell measurements of Hassel and Richter-Salvesen have been converted from $\mathrm{kX}$ to angstrom units for comparison with the NBS values.

Lattice constants

\begin{tabular}{|c|c|c|c|}
\hline \multirow{3}{*}{$\begin{array}{l}1927 \\
1962\end{array}$} & \multirow{3}{*}{$\begin{array}{l}\text { Hassel and Richter- } \\
\text { Salvesen [1] } \\
\text { National Bureau of } \\
\text { Standards at } 25^{\circ} \mathrm{C}_{-}\end{array}$} & $a$ & $c$ \\
\hline & & $\AA$ & $\AA$ \\
\hline & & 9. 370 & 9. 732 \\
\hline
\end{tabular}

The density of cobalt fluosilicate hexahydrate calculated from the NBS lattice constants is $2.081 \mathrm{~g} / \mathrm{cm}^{3}$ at $25^{\circ} \mathrm{C}$.

\begin{tabular}{|c|c|c|}
\hline \multirow{2}{*}{$\begin{array}{c}h k l \\
\text { (hex.) }\end{array}$} & \multicolumn{2}{|c|}{$\begin{array}{c}\text { Internal Standard, } \\
\text { Tungsten, } a=3.1648 \AA \\
\text { Co, } 1.7889 \AA \text { at } 25{ }^{\circ} \mathrm{C}\end{array}$} \\
\hline & $d$ & $I$ \\
\hline $\begin{array}{l}110 \\
102 \\
202 \\
211 \\
113\end{array}$ & $\begin{array}{l}\quad \stackrel{\AA}{4} \\
\text { 4. } 69 \\
\text { 4. } 18 \\
\text { 3. } 116 \\
2.927 \\
\text { 2. } 668\end{array}$ & $\begin{array}{r}100 \\
63 \\
5 \\
11 \\
5\end{array}$ \\
\hline $\begin{array}{l}122 \\
104 \\
311 \\
024 \\
303\end{array}$ & $\begin{array}{l}2.595 \\
2.331 \\
2.193 \\
2.086 \\
2.078\end{array}$ & $\begin{array}{r}29 \\
2 \\
3 \\
6\end{array}$ \\
\hline $\begin{array}{l}312 \\
401 \\
214 \\
223 \\
042\end{array}$ & $\begin{array}{l}2.042 \\
1.986 \\
1.907 \\
1.900 \\
1.872\end{array}$ & $\begin{array}{r}<2 \\
3 \\
12 \\
5\end{array}$ \\
\hline $\begin{array}{l}321 \\
410 \\
232 \\
134 \\
330\end{array}$ & $\begin{array}{l}1.829 \\
\text { 1. } 771 \\
\text { 1. } 738 \\
1.652 \\
1.562\end{array}$ & $\begin{array}{r}<2 \\
4 \\
3 \\
7 \\
<2\end{array}$ \\
\hline $\begin{array}{l}413 \\
502 \\
324 \\
422 \\
152\end{array}$ & $\begin{array}{l}1.555 \\
1.540 \\
1.479 \\
1.462 \\
1.397\end{array}$ & $\begin{array}{r}<2 \\
<2 \\
2 \\
<2 \\
2\end{array}$ \\
\hline $\begin{array}{l}306 \\
600 \\
226 \\
520 \\
244\end{array}$ & $\begin{array}{l}\text { 1. } 3916 \\
\text { 1. } 3524 \\
\text { 1. } 3335 \\
\text { 1. } 2993 \\
\text { 1. } 2969\end{array}$ & $\begin{array}{r}2 \\
<2 \\
2 \\
5 \\
4\end{array}$ \\
\hline $\begin{array}{c}342 \\
018 \\
416 \\
440 \\
532,072\end{array}$ & $\begin{array}{l}\text { 1. } 2868 \\
\text { 1. } 2028 \\
\text { 1. } 1963 \\
\text { 1. } 1712 \\
\text { 1. } 1279\end{array}$ & $\begin{array}{l}2 \\
<2 \\
<2 \\
<2\end{array}$ \\
\hline
\end{tabular}

\section{Reference}

[1] O. Hassel and J. Richter-Salvesen, Ưber den Kristallbau der trigonal kristallisierenden heteropolaren Verbindungen von der Zusammensetzung $\mathrm{MG}_{6} \cdot \mathrm{LR}_{6}$ und $\mathrm{MG}_{5} \mathrm{D} \cdot \mathrm{LR}_{6}$ und $\mathrm{MG}_{4} \mathrm{D}_{2} \mathrm{LR}_{6}, \mathrm{Z}$. physik. Chem. 128, 345-361 (1927). 


\section{Cobalt Perchlorate Hexahydrate, $\mathrm{Co}\left(\mathrm{ClO}_{4}\right)_{2} \cdot 6 \mathrm{H}_{2} \mathrm{O}$ (hexagonal)}

Powder data cards. None.

Additional published patterns. West [1] 1935.

NBS sample. The sample of cobalt perchlorate hexahydrate was obtained from the City Chemical Corp., New York, N.Y. Spectrographic analysis showed the following major impurities: 0.01 to 0.1 percent of nickel, and 0.001 to 0.01 percent each of copper, magnesium, and silicon.

The color of the sample was pink. It is optically negative with the indices of refraction $\mathrm{N}_{\mathrm{e}}=1.492$ and $\mathrm{N}_{\mathrm{o}}=1.512$.

The $d$-values of the three strongest lines are: $3.900,4.139$, and $2.840 \AA$.

Structural data. West [1] in 1935 determined that cobalt perchlorate hexahydrate has the magnesium perchlorate hexahydrate structure, the space group $\mathrm{D}_{6 \mathrm{~h}}^{1}-\mathrm{P} 6 / \mathrm{mmm}$ (No. 191), and $4\left[\mathrm{Co}\left(\mathrm{ClO}_{4}\right)_{2} \cdot 6 \mathrm{H}_{2} \mathrm{O}\right]$ per unit cell. The lattice constants of West have been converted from $\mathrm{kX}$ to angstrom units for comparison with the NBS values.

\section{Lattice constants}

\begin{tabular}{|c|c|c|c|}
\hline & & $a$ & $c$ \\
\hline $\begin{array}{l}1935 \\
1963\end{array}$ & $\begin{array}{l}\text { West [1] } \\
\text { National Bureau of } \\
\quad \text { Standards at } 26^{\circ} \mathrm{C} \text {. }\end{array}$ & $\begin{array}{l}\stackrel{\AA}{A} \\
\text { 15. } 55 \\
\text { 15. } 610 \\
\text { 土. } 002\end{array}$ & $\begin{array}{l}\text { Ă } \\
\text { 5. } 21 \\
\text { 5. } 2372 \\
\text { 士. } 0007\end{array}$ \\
\hline
\end{tabular}

The density of cobalt perchlorate hexahydrate calculated from the NBS lattice constants is $2.199 \pm 0.001 \mathrm{~g} / \mathrm{cm}^{3}$ at $26^{\circ} \mathrm{C}$.

\begin{tabular}{|c|c|c|}
\hline \multirow[t]{2}{*}{$h k l$} & \multicolumn{2}{|c|}{$\begin{array}{c}\text { Internal Standard, } \\
\text { Silver, } a=4.0861 \AA \\
\mathrm{Co}, 1.7889 \AA \text { at } 26{ }^{\circ} \mathrm{C}\end{array}$} \\
\hline & $d$ & $I$ \\
\hline $\begin{array}{l}200 \\
101 \\
111 \\
201 \\
220\end{array}$ & 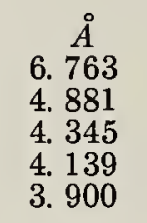 & $\begin{array}{r}3 \\
4 \\
3 \\
62 \\
100\end{array}$ \\
\hline $\begin{array}{l}211 \\
311 \\
401 \\
002 \\
420\end{array}$ & $\begin{array}{l}\text { 3. } 661 \\
\text { 3. } 052 \\
\text { 2. } 840 \\
\text { 2. } 618 \\
\text { 2. } 556\end{array}$ & $\begin{array}{r}2 \\
<2 \\
60 \\
8 \\
3\end{array}$ \\
\hline $\begin{array}{l}202 \\
421 \\
600 \\
402\end{array}$ & $\begin{array}{l}\text { 2. } 440 \\
\text { 2. } 296 \\
\text { 2. } 253 \\
2.069\end{array}$ & $\begin{array}{r}3 \\
10 \\
3 \\
8\end{array}$ \\
\hline $\begin{array}{l}440 \\
620\end{array}$ & $\begin{array}{l}\text { 1. } 952 \\
\text { 1. } 874\end{array}$ & $\begin{array}{l}9 \\
2\end{array}$ \\
\hline $\begin{array}{l}422 \\
441\end{array}$ & 1. 828 & 10 \\
\hline 621 & 1. 7655 & 6 \\
\hline $\begin{array}{l}203 \\
640 \\
641 \\
820\end{array}$ & $\begin{array}{l}\text { 1. } 6907 \\
\text { 1. } 5502 \\
\text { 1. } 4869 \\
\text { 1. } 4753\end{array}$ & $\begin{array}{l}2 \\
3 \\
2 \\
4\end{array}$ \\
\hline $\begin{array}{l}802 \\
821\end{array}$ & 1. 4201 & 2 \\
\hline $\begin{array}{l}443 \\
660\end{array}$ & 1. 3011 & 4 \\
\hline 224 & 1. 2413 & 2 \\
\hline
\end{tabular}

\section{Reference}

[1] C. D. West, The crystal structures of hydrated compounds II. Structure type $\mathrm{Mg}\left(\mathrm{ClO}_{4}\right)_{2} \cdot 6 \mathrm{H}_{2} \mathrm{O}$, Z. Krist. A s1, 480 (1935). 


\section{Copper Sulfate (chalcocyanite), $\mathrm{CuSO}_{4}$ (orthorhombic)}

\section{Powder data cards}

\begin{tabular}{|c|c|c|}
\hline $\begin{array}{c}\text { Card } \\
\text { number }\end{array}$ & $\begin{array}{c}\text { Index } \\
\text { lines }\end{array}$ & Source \\
\hline 12-779 & $\begin{array}{l}2.62 \\
4.20 \\
3.55\end{array}$ & $\begin{array}{c}\text { Hanawalt, Rinn, and Frevel } \\
\text { [1] 1938. }\end{array}$ \\
\hline
\end{tabular}

Additional published pattern. Pistorius [2] 1960.

NBS sample. The sample of copper sulfate was obtained from the NBS laboratories in Boulder, Colo. The sample was ground and fumed off with sulfuric acid and placed immediately in a dry atmosphere. Special care was necessary to keep it anhydrous. The sample was mounted in a holder which maintained a dry atmosphere while the patterns were run. Spectrographic analysis showed the following major impurities: 0.01 to 0.1 percent silicon, and 0.001 to 0.01 percent each of aluminum, iron, magnesium, and nickel.

The sample was colorless. The indices of refraction could not be obtained because the sample was too fine.

The $d$-values of the three strongest lines are: $3.549,(2.616$ and 2.611$)$, and $4.187 \AA$.

Structural data. Kokkoros and Rentzeperis [3] in 1958 determined that copper sulfate has the space group $\mathrm{D}_{2 \mathrm{~h}}^{16}-\mathrm{Pmnb}$ (No. 62) and $4\left(\mathrm{CuSO}_{4}\right)$ per unit cell. Rama Rao [4] in 1961 made refinements on the structure.

Lattice constants

\begin{tabular}{|c|c|c|c|c|}
\hline & & $a$ & $b$ & $c$ \\
\hline 1958 & $\begin{array}{l}\text { Kokkoros \& } \\
\text { Rentzeperis } \\
\text { [3]. }\end{array}$ & $\begin{array}{c}\stackrel{\circ}{A} \\
\text { 6. } 69\end{array}$ & $\begin{array}{r}\stackrel{\circ}{A} \\
\text { 8. } 39\end{array}$ & 4. $\begin{array}{c}\stackrel{\AA}{8} \\
\end{array}$ \\
\hline $\begin{array}{l}1960 \\
1963\end{array}$ & $\begin{array}{l}\text { Pistorius }[2] \\
\text { National Bureau } \\
\text { of Standards } \\
\text { at } 26^{\circ} \mathrm{C} \text {. }\end{array}$ & $\begin{array}{r}6.811 \\
6.6982 \\
\pm 0.0006\end{array}$ & $\begin{array}{r}\text { 8. } 391 \\
\text { 8. } 3956 \\
\pm .0006\end{array}$ & $\begin{array}{r}4.791 \\
4.8291 \\
+.0004\end{array}$ \\
\hline
\end{tabular}

\section{References}

[1] J. D. Hanawalt, H. W. Rinn, and L. K. Frevel, Chemical analysis by x-ray diffraction, Ind. Eng. Chem. Anal. Ed. 10, 457-512 (1938).

[2] C. W. F. T. Pistorius, Lattice constants and probable space group of anhydrous cupric sulfate (artificial chalcocyanite), Am. Mineralogist 45, 744-746 (1960).

[3] P. A. Kokkoros and P. J. Rentzeperis, The crystal structure of the anhydrous sulfates of copper and zinc, Acta Cryst. 11, 361 (1958).

[4] B. Rama Rao, A note on the crystal structure of anhydrous copper sulfate, Acta Cryst. 14, 321 (1961).
The density of copper sulfate calculated from the NBS lattice constants is $3.903 \mathrm{~g} / \mathrm{cm}^{3}$ at $26^{\circ} \mathrm{C}$.

\begin{tabular}{|c|c|c|}
\hline \multirow[t]{2}{*}{$h k l$} & \multicolumn{2}{|c|}{$\begin{array}{c}\text { Internal Standard, } \\
\text { Silver, } a=4.0861 \AA \\
\mathrm{Cu}, 1.5405 \AA \text { at } 26^{\circ} \mathrm{C}\end{array}$} \\
\hline & $d$ & $I$ \\
\hline $\begin{array}{l}011 \\
101 \\
111 \\
200 \\
021\end{array}$ & $\begin{array}{l}\stackrel{\AA}{1} \\
\text { 4. } 187 \\
\text { 3. } 921 \\
\text { 3. } 549 \\
\text { 3. } 346 \\
\text { 3. } 172\end{array}$ & $\begin{array}{r}77 \\
6 \\
100 \\
3 \\
4\end{array}$ \\
\hline $\begin{array}{l}121 \\
220 \\
211 \\
031 \\
002\end{array}$ & $\begin{array}{l}\text { 2. } 862 \\
\text { 2. } 616 \\
\text { 2. } 611 \\
\text { 2. } 421 \\
\text { 2. } 416\end{array}$ & $\begin{array}{r}2 \\
95\end{array}$ \\
\hline $\begin{array}{l}012 \\
221 \\
022 \\
301 \\
140\end{array}$ & $\begin{array}{l}\text { 2. } 321 \\
\text { 2. } 301 \\
\text { 2. } 093 \\
\text { 2. } 027 \\
\text { 2. } 003\end{array}$ & $\begin{array}{r}10 \\
12 \\
7 \\
3 \\
4\end{array}$ \\
\hline $\begin{array}{c}320,311 \\
231 \\
222 \\
400 \\
042\end{array}$ & $\begin{array}{l}\text { 1. } 971 \\
\text { 1. } 963 \\
\text { 1. } 7749 \\
\text { 1. } 6749 \\
\text { 1. } 5843\end{array}$ & $\begin{array}{r}9 \\
15 \\
28 \\
12 \\
11\end{array}$ \\
\hline $\begin{array}{l}013 \\
103 \\
420 \\
151 \\
341\end{array}$ & $\begin{array}{l}\text { 1. } 5806 \\
\text { 1. } 5650 \\
\text { 1. } 5553 \\
\text { 1. } 5436 \\
\text { 1. } 4578\end{array}$ & $\begin{array}{r}10 \\
2 \\
8 \\
3 \\
<1\end{array}$ \\
\hline $\begin{array}{l}251 \\
213 \\
060 \\
402 \\
422 \\
303\end{array}$ & $\begin{array}{l}\text { 1. } 4330 \\
\text { 1. } 4298 \\
\text { 1. } 3996 \\
\text { 1. } 3760 \\
\text { 1. } 3073 \\
\text { 1. } 3056\end{array}$ & $\begin{array}{r}22 \\
18 \\
4 \\
12 \\
5\end{array}$ \\
\hline $\begin{array}{c}342 \\
313 \\
520,511 \\
062 \\
004\end{array}$ & $\begin{array}{l}\text { 1. } 2919 \\
\text { 1. } 2902 \\
\text { 1. } 2763 \\
\text { 1. } 2107 \\
\text { 1. } 2075\end{array}$ & $\begin{array}{l}3 \\
4 \\
3 \\
2\end{array}$ \\
\hline $\begin{array}{l}014 \\
162 \\
442 \\
413 \\
214\end{array}$ & $\begin{array}{l}\text { 1. } 1948 \\
\text { 1. } 1918 \\
\text { 1. } 1508 \\
\text { 1. } 1496 \\
\text { 1. } 1258\end{array}$ & $\begin{array}{l}2 \\
3 \\
3\end{array}$ \\
\hline $\begin{array}{c}224 \\
620,611 \\
433 \\
371 \\
181,631\end{array}$ & $\begin{array}{l}\text { 1. } 0964 \\
\text { 1. } 0790 \\
\text { 1. } 0721 \\
\text { 1. } 0323 \\
\text { 1. } 0138\end{array}$ & $\begin{array}{r}4 \\
5 \\
4 \\
<1 \\
1\end{array}$ \\
\hline $\begin{array}{l}244 \\
622 \\
462 \\
424 \\
282\end{array}$ & $\begin{array}{r}0.9990 \\
.9850 \\
.9812 \\
.9536 \\
.9249\end{array}$ & $\begin{array}{l}3 \\
3 \\
4 \\
3 \\
2\end{array}$ \\
\hline
\end{tabular}




\section{Dysprosium Arsenate, $\mathrm{DyAsO}_{4}$ (tetragonal)}

Powder data cards. None.

Additional published patterns. None.

NBS sample. The sample of dysprosium arsenate was prepared at NBS from a water solution of dysprosium trichloride and arsenic pentoxide. The sample was dried at $110{ }^{\circ} \mathrm{C}$. Spectrographic analysis showed the following major impurities: 0.01 to 0.1 percent antimony and 0.001 to 0.01 percent each of calcium, magnesium, lead, and silicon.

The sample is colorless. The indices of refraction could not be determined because the sample was too fine.

The $d$-values of the three strongest lines are: $3.537,2.669$, and $1.8246 \AA$.

Structural data. Durif and Forrat [1] in 1957 determined that dysprosium arsenate has the zircon structure with the space group $\mathrm{D}_{4 \mathrm{~h}}^{19}-I 4_{1} /$ amd (No. 141) and 4[DyAsO 4 ] per unit cell.

Lattice constants

\begin{tabular}{|c|c|c|c|}
\hline & & $a$ & $c$ \\
\hline $\begin{array}{l}1957 \\
1963\end{array}$ & $\begin{array}{l}\text { Durif and Forrat }[1] \\
\text { National Bureau of } \\
\text { Standards at } 25^{\circ} \mathrm{C} \text {. }\end{array}$ & $\begin{array}{c}\stackrel{\AA}{A} \\
7.09 \\
7.0733 \\
\pm 0.0003\end{array}$ & $\begin{array}{c}\AA \\
\text { 6. } 315 \\
6.3133 \\
\pm 0.0003\end{array}$ \\
\hline
\end{tabular}

The density of dysprosium arsenate calculated from the NBS lattice constants is $6.338 \mathrm{~g} / \mathrm{cm}^{3}$ at $25^{\circ} \mathrm{C}$.

\section{Reference}

[1] A. Durif and F. Forrat, Sur quelques arséniates des terres rares à structure zircon, Compt. Rend. $\mathbf{2 4 5}$, 1636-38 (1957).

\begin{tabular}{|c|c|c|}
\hline \multirow[t]{2}{*}{$h k l$} & \multicolumn{2}{|c|}{$\begin{array}{c}\text { Internal Standard, } \\
\text { Silver, } a=4.0861 \AA \\
\text { Co, } 1.7889 \AA \text { at } 25^{\circ} \mathrm{C}\end{array}$} \\
\hline & $d$ & $I$ \\
\hline $\begin{array}{l}101 \\
200 \\
211 \\
112 \\
220\end{array}$ & $\begin{array}{c}\stackrel{\AA}{A} \\
\text { 4. } 713 \\
\text { 3. } 537 \\
\text { 2. } 828 \\
\text { 2. } 669 \\
\text { 2. } 500\end{array}$ & $\begin{array}{r}15 \\
100 \\
3 \\
67 \\
22\end{array}$ \\
\hline $\begin{array}{l}301 \\
103 \\
321 \\
312 \\
400\end{array}$ & $\begin{array}{l}\text { 2. } 209 \\
\text { 2. } 017 \\
\text { 1. } 873 \\
\text { 1. } 8246 \\
\text { 1. } 7631\end{array}$ & $\begin{array}{r}8 \\
8 \\
5 \\
47 \\
14\end{array}$ \\
\hline $\begin{array}{l}213 \\
411 \\
420 \\
303 \\
332\end{array}$ & $\begin{array}{l}\text { 1. } 7522 \\
\text { 1. } 6556 \\
\text { 1. } 5812 \\
\text { 1. } 5702 \\
\text { 1. } 4740\end{array}$ & $\begin{array}{r}3 \\
<3 \\
14 \\
4 \\
14\end{array}$ \\
\hline $\begin{array}{l}204 \\
501 \\
224 \\
512 \\
440\end{array}$ & $\begin{array}{l}\text { 1. } 4413 \\
\text { 1. } 3802 \\
\text { 1. } 3345 \\
\text { 1. } 2701 \\
\text { 1. } 2504\end{array}$ & $\begin{array}{r}12 \\
<3 \\
10 \\
<3\end{array}$ \\
\hline $\begin{array}{l}600 \\
404 \\
532 \\
424 \\
631\end{array}$ & $\begin{array}{l}\text { 1. } 1790 \\
\text { 1. } 1773 \\
\text { 1. } 1323 \\
\text { 1. } 1172 \\
\text { 1. } 0400\end{array}$ & $\begin{array}{r}6 \\
7 \\
6 \\
11 \\
3\end{array}$ \\
\hline $\begin{array}{l}116 \\
415 \\
444 \\
552 \\
316\end{array}$ & $\begin{array}{r}\text { 1. } 0297 \\
\text { 1. } 0171 \\
\text { 0. } 9803 \\
.9535 \\
.9521\end{array}$ & $\begin{array}{r}5 \\
3 \\
7 \\
9 \\
10\end{array}$ \\
\hline $\begin{array}{l}604 \\
624\end{array}$ & $\begin{array}{l}9444 \\
.9126\end{array}$ & $\begin{array}{l}4 \\
9\end{array}$ \\
\hline
\end{tabular}


Powder data cards. None.

Additional published patterns. None.

NBS sample. The sample of erbium arsenate was prepared at NBS from a water solution of arsenic pentoxide and erbium trichloride. Spectrographic analysis showed the following major impurities: 0.01 to 0.1 percent each of nickel and antimony and 0.001 to 0.01 percent each of aluminum, calcium, magnesium, lead, and silicon.

The sample was very pale pink. The indices of refraction could not be determined because the sample was too fine.

The $d$-values of the three strongest lines are: $3.510,2.652$, and $1.812 \AA$.

Structural data. Durif and Forrat [1] in 1957 determined that erbium arsenate has the zircon structure with the space group $\mathrm{D}_{4 \mathrm{~h}}^{19}-\mathrm{I} 4_{1} /$ amd (No. 141) and $4\left(\mathrm{ErAsO}_{4}\right)$ per unit cell.

\section{Lattice constants}

\begin{tabular}{|c|c|c|c|}
\hline & & $a$ & $c$ \\
\hline $\begin{array}{l}1957 \\
1963\end{array}$ & $\begin{array}{l}\text { Durif and Forrat [1] } \\
\text { National Bureau of } \\
\text { Standards at } 25^{\circ} \mathrm{C} .\end{array}$ & $\begin{array}{l}\stackrel{ }{\AA} \\
7.04 \\
7.0203 \\
\pm .0002\end{array}$ & $\begin{array}{l}\stackrel{\AA}{A} \\
\text { 6. } 30 \\
\text { 6. } 2761 \\
\text { 土. } 0004\end{array}$ \\
\hline
\end{tabular}

The density of erbium arsenate calculated from the NBS lattice constants is $6.574 \mathrm{~g} / \mathrm{cm}^{3}$ at $25^{\circ} \mathrm{C}$.

\section{Reference}

[1] A. Durif and F. Forrat, Sur quelques arséniates des terres rares à structure zircon, Compt. Rend. 245, 1636-38 (1957).

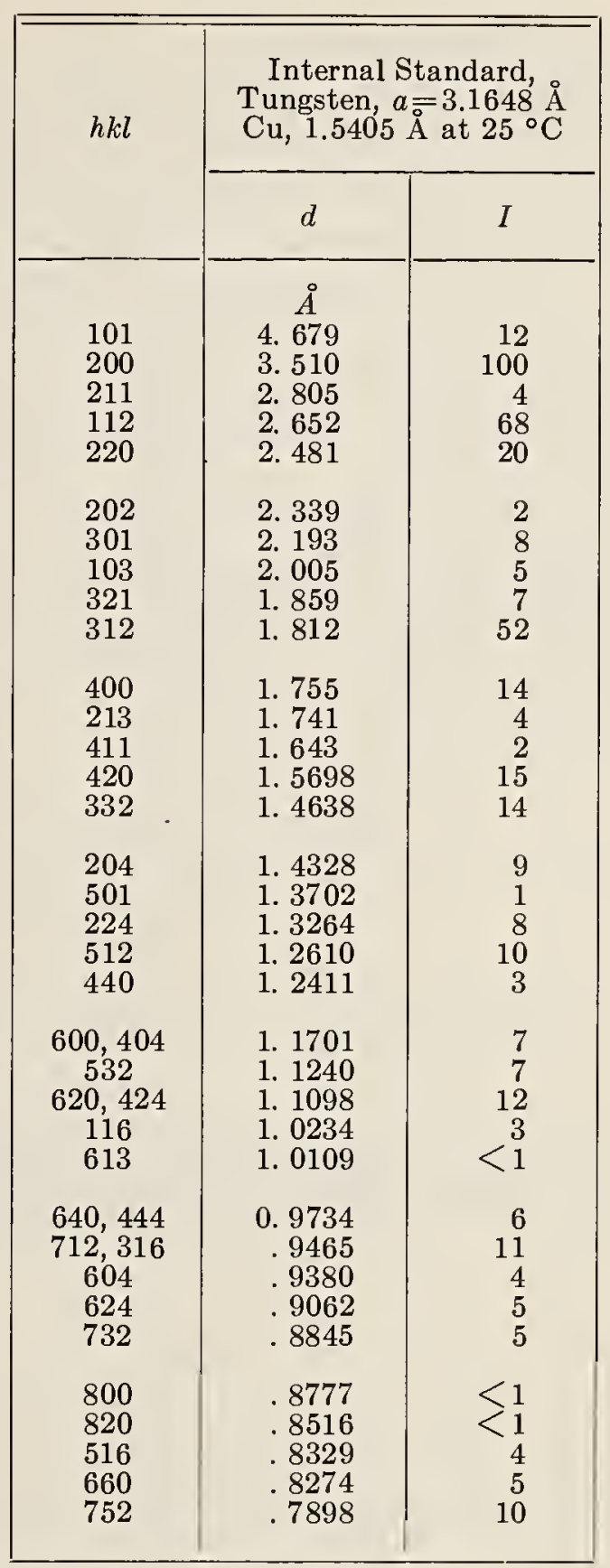




\section{Europium Arsenate, $\mathrm{EuAsO}_{4}$ (tetragonal)}

Powder data cards. None.

Additional published patterns. None.

NBS sample. The sample of europium arsenate was prepared at NBS from a water solution of arsenic pentoxide and europium trichloride. It was dried at $110{ }^{\circ} \mathrm{C}$. Spectrographic analysis showed the following major impurities: 0.01 to 0.1 percent each of antimony and silicon; 0.001 to 0.01 percent each of aluminum, calcium, iron, magnesium, and titanium.

The sample was colorless. The indices of refraction could not be determined because the sample was too fine.

The $d$-values of the three strongest lines are: $3.578,2.704$, and $1.8467 \AA$

Structural data. No reference to the structure of europium arsenate was found but it is apparently isostructural with yttrium arsenate with the space group $\mathrm{D}_{4 \mathrm{~h}}^{19}-\mathrm{I}_{1} /$ amd (No. 141) and $4\left(\mathrm{EuAsO}_{4}\right)$ per unit cell.

\section{Lattice Constants}

\begin{tabular}{|c|c|c|c|}
\hline & & $a$ & $c$ \\
\hline 1963 & $\begin{array}{l}\text { National Bureau of } \\
\text { Standards at } 25^{\circ} \mathrm{C} \text {. }\end{array}$ & $\begin{array}{c}\stackrel{\AA}{1} \\
\text { 7. } 1541 \\
\pm .0004\end{array}$ & $\begin{array}{c}\stackrel{\AA}{A} \\
\text { 6. } 3953 \\
\pm .0004\end{array}$ \\
\hline
\end{tabular}

The density of europium arsenate calculated from the NBS lattice constants is $5.902 \mathrm{~g} / \mathrm{cm}^{3}$ at $25^{\circ} \mathrm{C}$.

\begin{tabular}{|c|c|c|}
\hline \multirow[t]{2}{*}{$h k l$} & \multicolumn{2}{|c|}{$\begin{array}{l}\text { Internal Standard, } \\
\text { Tungsten, } a=3.1648 \AA \\
\text { Co, } 1.7889 \AA \text { at } 25^{\circ} \mathrm{C}\end{array}$} \\
\hline & $d$ & $I$ \\
\hline $\begin{array}{l}101 \\
200 \\
112 \\
220 \\
202\end{array}$ & $\begin{array}{l}\stackrel{\AA}{A} \\
\text { 4. } 77 \\
\text { 3. } 578 \\
\text { 2. } 704 \\
\text { 2. } 530 \\
\text { 2. } 386\end{array}$ & $\begin{array}{r}2 \\
100 \\
63 \\
17 \\
5\end{array}$ \\
\hline $\begin{array}{l}301 \\
103 \\
321 \\
312 \\
400\end{array}$ & $\begin{array}{l}\text { 2. } 236 \\
\text { 2. } 0430 \\
\text { 1. } 8953 \\
\text { 1. } 8467 \\
\text { 1. } 7886\end{array}$ & $\begin{array}{r}5 \\
3 \\
5 \\
45 \\
12\end{array}$ \\
\hline $\begin{array}{l}420 \\
332 \\
204 \\
224 \\
512\end{array}$ & $\begin{array}{l}\text { 1. } 5997 \\
\text { 1. } 4914 \\
\text { 1. } 4595 \\
\text { 1. } 3511 \\
\text { 1. } 2847\end{array}$ & $\begin{array}{r}11 \\
12 \\
8 \\
8 \\
8\end{array}$ \\
\hline $\begin{array}{c}440 \\
600,404 \\
532 \\
424 \\
116\end{array}$ & $\begin{array}{l}\text { 1. } 2646 \\
\text { 1. } 1921 \\
\text { 1. } 1455 \\
\text { 1. } 1308 \\
\text { 1. } 0428\end{array}$ & $\begin{array}{r}3 \\
7 \\
8 \\
10 \\
5\end{array}$ \\
\hline $\begin{array}{l}444 \\
316 \\
604 \\
624\end{array}$ & $\begin{array}{r}0.9919 \\
.9643 \\
.9557 \\
.9233\end{array}$ & $\begin{array}{r}5 \\
11 \\
3 \\
6\end{array}$ \\
\hline
\end{tabular}




\section{Gallium Arsenide, GaAs (cubic)}

Powder data cards. None.

Additional published patterns. None.

NBS sample. The sample of gallium arsenide was obtained from Semitronics Inc., Winchester, Mass. Spectrographic analysis showed the following major impurities: 0.1 to 1.0 percent iron, 0.01 to 0.1 percent chromium, nickel, and lead; and 0.001 to 0.01 percent each of cobalt, copper, indium, manganese, molybdenum, and silicon.

The sample was a dark gray opaque powder.

The $d$-values of the three strongest lines are: $3.262,1.998$, and $1.704 \AA$.

Structural data. Goldschmidt [1] in 1927 determined that gallium arsenide has the zinc sulfide structure, the space group $\mathrm{T}_{\mathrm{d}}^{2}-\overline{\mathrm{F}} \overline{4} 3 \mathrm{~m}$ (No. 216), and $4(\mathrm{GaAs})$ per unit cell.

\section{Lattice constant}

\begin{tabular}{|c|c|c|}
\hline 1963 & $\begin{array}{c}\text { National Bureau of Standards at } \\
25^{\circ} \mathrm{C} \ldots \ldots \ldots\end{array}$ & $\begin{array}{c}\AA \\
\text { 5. } 652\end{array}$ \\
\hline
\end{tabular}

The density of gallium arsenide calculated from the NBS lattice constant is $5.321 \mathrm{~g} / \mathrm{cm}^{3}$ at $25^{\circ} \mathrm{C}$.

\begin{tabular}{|c|c|c|c|}
\hline \multirow[t]{2}{*}{$h k l$} & \multicolumn{3}{|c|}{$\begin{array}{l}\text { Internal Standard, } \\
\text { Tungsten, } a=3.1648 \AA \\
\mathrm{Cu}, 1.5405 \AA \text { at } 25^{\circ} \mathrm{C}\end{array}$} \\
\hline & $d$ & $I$ & $a$ \\
\hline $\begin{array}{l}111 \\
220 \\
311 \\
400 \\
331\end{array}$ & $\begin{array}{l}\stackrel{\AA}{A} \\
\text { 3. } 262 \\
1.998 \\
1.704 \\
1.413 \\
1.297\end{array}$ & $\begin{array}{r}100 \\
61 \\
29 \\
7 \\
11\end{array}$ & $\begin{array}{c}\stackrel{\AA}{A} \\
\text { 5. } 648 \\
\text { 5. } 650 \\
\text { 5. } 650 \\
\text { 5. } 648 \\
\text { 5. } 652\end{array}$ \\
\hline $\begin{array}{l}422 \\
511 \\
440 \\
531 \\
620\end{array}$ & $\begin{array}{l}1.154 \\
1.088 \\
0.9993 \\
.9557 \\
.8938\end{array}$ & $\begin{array}{r}17 \\
5 \\
3 \\
6 \\
6\end{array}$ & $\begin{array}{l}\text { 5. } 647 \\
\text { 5. } 648 \\
\text { 5. } 652 \\
\text { 5. } 653 \\
\text { 5. } 652\end{array}$ \\
\hline $\begin{array}{l}533 \\
444 \\
711\end{array}$ & $\begin{array}{l}.8621 \\
.8159 \\
.7916\end{array}$ & $\begin{array}{l}4 \\
2 \\
6\end{array}$ & $\begin{array}{l}\text { 5. } 652 \\
\text { 5. } 652 \\
\text { 5. } 652\end{array}$ \\
\hline \multicolumn{3}{|c|}{ Average value of last five lines. } & 5. 652 \\
\hline
\end{tabular}

\section{Reference}

[1] V. M. Goldschmidt, Geochemische Verteilungsgesetze VIII, Skrifter Norske Vidensk. Akad. Oslo, Math. Nat. K1. 8, 390 (1926-27). 
Powder data cards. None.

Additional published patterns. None.

NBS sample. The sample of holmium arsenate was prepared at NBS from a water solution of arsenic pentoxide and holmium trichloride. Spectrographic analysis showed the following major impurities: 0.01 to 0.1 percent antimony and 0.001 to 0.01 percent each of aluminum, calcium, iron, lead, and silicon.

The color of the sample was very pale pinkish white. The indices of refraction could not be determined because the sample was too fine.

The $d$-values of the three strongest lines are: $3.528,2.668$, and $1.822 \AA$.

Structural data. The structure of holmium arsenate was not found in the literature; however, it is apparently isostructural with erbium arsenate having the space group $\mathrm{D}_{4 \mathrm{~h}}^{19}-\mathrm{I} 4_{1} /$ amd (No. 141) and $4\left(\mathrm{HoAsO}_{4}\right)$ per uiitit cell.

\section{Lattice constants}

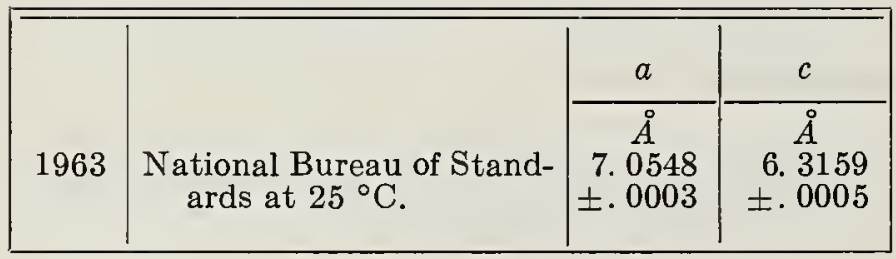

The density of holmium arsenate calculated from NBS lattice constants is $6.420 \mathrm{~g} / \mathrm{cm}^{3}$ at $25^{\circ} \mathrm{C}$.

\begin{tabular}{|c|c|c|}
\hline \multirow[t]{2}{*}{$h k l$} & \multicolumn{2}{|c|}{$\begin{array}{c}\text { Internal Standard, } \\
\text { Tungsten, } a=3.1648 \AA \\
\mathrm{Cu} 1.5405 \AA \text { at } 25^{\circ} \mathrm{C}\end{array}$} \\
\hline & $d$ & $I$ \\
\hline $\begin{array}{l}101 \\
200 \\
211 \\
112 \\
220\end{array}$ & $\begin{array}{c}\stackrel{\AA}{A} \\
\text { 4. } 709 \\
\text { 3. } 528 \\
2.819 \\
2.668 \\
2.494\end{array}$ & $\begin{array}{r}5 \\
100 \\
4 \\
67 \\
20\end{array}$ \\
\hline $\begin{array}{l}202 \\
301 \\
103 \\
321 \\
312\end{array}$ & $\begin{array}{l}2.352 \\
2.203 \\
2.017 \\
1.868 \\
1.822\end{array}$ & $\begin{array}{r}6 \\
7 \\
5 \\
4 \\
50\end{array}$ \\
\hline $\begin{array}{l}400 \\
420 \\
332 \\
204 \\
501\end{array}$ & $\begin{array}{l}\text { 1. } 763 \\
\text { 1. } 5774 \\
\text { 1. } 4710 \\
\text { 1. } 4411 \\
\text { 1. } 3769\end{array}$ & $\begin{array}{r}16 \\
14 \\
15 \\
9 \\
1\end{array}$ \\
\hline $\begin{array}{c}224 \\
413 \\
512 \\
440 \\
404,600\end{array}$ & $\begin{array}{l}\text { 1. } 3339 \\
\text { 1. } 3279 \\
\text { 1. } 2670 \\
\text { 1. } 2470 \\
\text { 1. } 1763\end{array}$ & $\begin{array}{l}9 \\
4 \\
9 \\
3 \\
5\end{array}$ \\
\hline $\begin{array}{l}532 \\
424 \\
116 \\
206 \\
640\end{array}$ & $\begin{array}{l}\text { 1. } 1299 \\
1.1159 \\
1.0301 \\
\text { 1. } 0086 \\
0.9781\end{array}$ & $\begin{array}{r}6 \\
10 \\
3 \\
1 \\
4\end{array}$ \\
\hline $\begin{array}{l}552 \\
604 \\
624 \\
732 \\
800\end{array}$ & $\begin{array}{l}.9516 \\
.9428 \\
.9110 \\
.8890 \\
.8818\end{array}$ & $\begin{array}{r}7 \\
2 \\
3 \\
4 \\
<1\end{array}$ \\
\hline $\begin{array}{l}820 \\
516 \\
644 \\
660\end{array}$ & $\begin{array}{l}.8556 \\
.8378 \\
.8317 \\
.8314\end{array}$ & $\begin{array}{l}1 \\
2 \\
3 \\
2\end{array}$ \\
\hline
\end{tabular}




\section{Indium Arsenide, InAs (cubic)}

Powder data cards

\begin{tabular}{|c|c|c|}
\hline $\begin{array}{c}\text { Card } \\
\text { number }\end{array}$ & $\begin{array}{c}\text { Index } \\
\text { lines }\end{array}$ & Source \\
\hline $8-387$ & $\begin{array}{r}1.24 \\
1.02 \\
2.10\end{array}$ & Liu and Peretti [1] 1953. \\
\hline
\end{tabular}

1941

Additional published patterns. A. Iandelli [2]

NBS sample. The sample of indium arsenide was obtained from Semitronics, Inc., Winchester Mass. Spectrographic analysis showed the following impurities: 0.01 to 0.1 percent each of silver, aluminum, bismuth, chromium, iron, gallium, and titanium; and 0.001 to 0.01 percent each of barium, calcium, cobalt, magnesium, nickel, antimony, and tin.

The sample was an opaque metallic powder.

The $d$-values of the three strongest lines are: $3.498,2.142$, and $1.8263 \AA$.

Structural data. Iandelli [2] in 1941 determined that indium arsenide has the zinc sulfide structure, the space group $\mathrm{T}_{\mathrm{d}}^{2}-\mathrm{F} \overline{4} 3 \mathrm{~m}$ (No. 216), and $4(\mathrm{InAs})$ per unit cell. The unit cell measurement reported by Iandelli has been converted from $\mathrm{kX}$ to angstrom units for comparison with the NBS value.

\section{Lattice constants}

\begin{tabular}{|c|c|c|}
\hline $\begin{array}{l}1941 \\
1953 \\
1963\end{array}$ & $\begin{array}{l}\text { Iandelli }[2] \\
\text { Liu and Peretti }[1] \\
\text { National Bureau of Standards at } \\
25{ }^{\circ} \mathrm{C}\end{array}$ & $\begin{array}{l}\stackrel{\circ}{A} \\
\text { 6. } 048 \\
\text { 6. } 058 \\
\text { 6. } 058\end{array}$ \\
\hline
\end{tabular}

The density of indium arsenide calculated from NBS lattice constant is $5.668 \mathrm{~g} / \mathrm{cm}^{3}$ at $25^{\circ} \mathrm{C}$.

\begin{tabular}{|c|c|c|c|}
\hline \multirow{2}{*}{$h k l$} & \multicolumn{3}{|c|}{$\begin{array}{l}\text { Internal Standard, Tungsten, } a=3.1648 \AA \\
\qquad \mathrm{Cu}, 1.5405 \AA \text { at } 25^{\circ} \mathrm{C}\end{array}$} \\
\hline & $d$ & $I$ & $a$ \\
\hline $\begin{array}{l}111 \\
200 \\
220 \\
311 \\
222\end{array}$ & $\begin{array}{ll} & \AA \\
\text { 3. } 498 \\
\text { 3. } 030 \\
\text { 2. } 142 \\
\text { 1. } 8263 \\
\text { 1. } 7489\end{array}$ & $\begin{array}{r}100 \\
8 \\
61 \\
38 \\
2\end{array}$ & $\begin{array}{l}\stackrel{\AA}{A} \\
6.059 \\
6.060 \\
6.059 \\
6.057 \\
6.058\end{array}$ \\
\hline $\begin{array}{l}400 \\
331 \\
420 \\
422 \\
511\end{array}$ & $\begin{array}{l}\text { 1. } 5145 \\
\text { 1. } 3895 \\
\text { 1. } 3544 \\
\text { 1. } 2366 \\
\text { 1. } 1658\end{array}$ & $\begin{array}{r}9 \\
13 \\
2 \\
15 \\
9\end{array}$ & $\begin{array}{l}\text { 6. } 058 \\
\text { 6. } 057 \\
\text { 6. } 057 \\
\text { 6. } 058 \\
\text { 6. } 058\end{array}$ \\
\hline $\begin{array}{l}440 \\
531 \\
600 \\
620 \\
533\end{array}$ & $\begin{array}{r}1.0707 \\
1.0241 \\
1.0097 \\
0.9578 \\
.9239\end{array}$ & $\begin{array}{r}7 \\
10 \\
1 \\
6 \\
3\end{array}$ & $\begin{array}{l}\text { 6. } 057 \\
\text { 6. } 058 \\
\text { 6. } 058 \\
\text { 6. } 058 \\
\text { 6. } 058\end{array}$ \\
\hline $\begin{array}{l}444 \\
711 \\
642 \\
731\end{array}$ & $\begin{array}{l}.8745 \\
.8483 \\
.8096 \\
.7887\end{array}$ & $\begin{array}{r}3 \\
6 \\
15 \\
8\end{array}$ & $\begin{array}{l}\text { 6. } 059 \\
6.058 \\
6.058 \\
6.058\end{array}$ \\
\hline
\end{tabular}

Average value of last five lines

6. 058

\section{References}

[1] T. S. Liu and E. A. Peretti, The indium-arsenic system, Trans. Am. Soc. Metals 45, 677-85 (1953).

[2] A. Iandelli, Sulla struttura dei composti, InP, InAs, e InSb, Gazz. chim. ital. 71, 58-62 (1941). 


\section{Lanthanum Arsenate, $\mathrm{LaAsO}_{4}$ (monoclinic)}

Powder data cards. None.

Additional published patterns. None.

NBS sample. The sample of lanthanum arsenate was prepared at NBS from a water solution of arsenic pentoxide and lanthanum trichloride. It was dried at $110^{\circ} \mathrm{C}$. Spectrographic analysis showed the following major impurities: 0.01 to 0.1 percent each of aluminum, iron, and silicon, and 0.001 to 0.01 percent each of calcium, magnesium, lead, and antimony.
The sample was colorless. The indices of refraction could not be determined because the sample was too fine.

The $d$-values of the three strongest lines are: $3.185,2.983$, and $3.391 \mathrm{~A}$.

Structural data. No reference to the structure of lanthanum arsenate was found; however, it is apparently isostructural with lanthanum phosphate with the space group $\mathrm{C}_{2 \mathrm{~h}}^{3}-\mathrm{P} 2_{1} / \mathrm{n}$ (No. 14) and $4\left(\mathrm{LaAsO}_{4}\right)$ per unit cell.

Lattice constants

\begin{tabular}{|c|c|c|c|c|c|}
\hline & & $a$ & $b$ & $c$ & $\beta$ \\
\hline 1963 & $\begin{array}{l}\text { National Bureau of Standards } \\
\text { at } 25^{\circ} \mathrm{C} \text {. }\end{array}$ & $\begin{array}{c}\stackrel{\AA}{A} \\
\text { 7. } 0078 \\
\pm .0007\end{array}$ & $\begin{array}{c}\stackrel{\circ}{1} \\
\text { 7. } 212 \\
\pm .001\end{array}$ & $\begin{array}{c}\AA \\
\text { 6. } 7670 \\
\pm .0007\end{array}$ & $\begin{array}{r}104^{\circ} 29.3^{\prime} \\
\pm .6^{\prime}\end{array}$ \\
\hline
\end{tabular}

The density of lanthanum arsenate calculated from the NBS lattice constants is $5.572 \mathrm{~g} / \mathrm{cm}^{3}$ at $25^{\circ} \mathrm{C}$.

\begin{tabular}{|c|c|c|c|c|c|}
\hline \multirow[t]{2}{*}{$h k l$} & \multicolumn{2}{|c|}{$\begin{array}{c}\text { Internal Standard, } \\
\text { Silver, } a=4.0861 \AA \\
\mathrm{Cu}, 1.5405 \AA \text { at } 25^{\circ} \mathrm{C}\end{array}$} & \multirow[t]{2}{*}{$h k l$} & \multicolumn{2}{|c|}{$\begin{array}{l}\text { Internal Standard, } \\
\text { Silver, } a=4.0861 \AA \\
\mathrm{Cu}, 1.5405 \AA \text { at } 25^{\circ} \mathrm{C}\end{array}$} \\
\hline & $d$ & $I$ & & $d$ & $I$ \\
\hline $\begin{array}{l}101 \\
110 \\
011 \\
111 \\
101\end{array}$ & $\begin{array}{l}\quad \AA \\
5.45 \\
4.95 \\
4.85 \\
4.35 \\
4.215\end{array}$ & $\begin{array}{r}4 \\
12 \\
10 \\
8 \\
<1\end{array}$ & $\begin{array}{c}\overline{3} 22 \\
231, \overline{2} 23 \\
040 \\
132 \\
140,321\end{array}$ & $\begin{array}{l}\stackrel{\AA}{1} \\
\text { 1. } 832 \\
1.808 \\
1.803 \\
1.795 \\
1.743\end{array}$ & $\begin{array}{r}20 \\
6 \\
5 \\
22 \\
11\end{array}$ \\
\hline $\begin{array}{l}111 \\
020 \\
200 \\
002 \\
120\end{array}$ & $\begin{array}{l}\text { 3. } 635 \\
\text { 3. } 606 \\
\text { 3. } 391 \\
\text { 3. } 278 \\
\text { 3. } 185\end{array}$ & $\begin{array}{r}7 \\
16 \\
60 \\
8 \\
100\end{array}$ & $\begin{array}{c}400 \\
402 \\
410 \\
330, \overline{1} 14 \\
004\end{array}$ & $\begin{array}{l}\text { 1. } 696 \\
\text { 1. } 689 \\
\text { 1. } 651 \\
\text { 1. } 647 \\
\text { 1. } 638\end{array}$ & $\begin{array}{r}4 \\
7 \\
11 \\
11 \\
5\end{array}$ \\
\hline $\begin{array}{l}210 \\
012 \\
\frac{2}{2} 12 \\
112\end{array}$ & $\begin{array}{l}\text { 3. } 068 \\
\text { 2. } 983 \\
2.722 \\
2.546 \\
\text { 2. } 527\end{array}$ & $\begin{array}{l}24 \\
87 \\
24 \\
22 \\
15\end{array}$ & $\begin{array}{c}\frac{312}{2} 14 \\
\overline{3} 32,240 \\
\overline{1} 42 \\
\overline{1} 24\end{array}$ & $\begin{array}{l}\text { 1. } 632 \\
\text { 1. } 604 \\
1.593 \\
\text { 1. } 581 \\
1.531\end{array}$ & $\begin{array}{l}4 \\
7 \\
8 \\
2 \\
6\end{array}$ \\
\hline $\begin{array}{l}220 \\
122 \\
\frac{1}{301} \\
031 \\
103\end{array}$ & $\begin{array}{l}\text { 2. } 471 \\
2.430 \\
2.326 \\
2.257 \\
2.252\end{array}$ & $\begin{array}{r}6 \\
8 \\
6 \\
11 \\
10\end{array}$ & $\begin{array}{l}322 \\
411 \\
\overline{4} 13 \\
\overline{2} 42 \\
\overline{3} 14\end{array}$ & $\begin{array}{l}\text { 1. } 519 \\
\text { 1. } 516 \\
\text { 1. } 504 \\
\text { 1. } 502 \\
\text { 1. } 487\end{array}$ & $\begin{array}{l}2 \\
1 \\
2 \\
2 \\
3\end{array}$ \\
\hline $\begin{array}{c}\overline{3} 11 \\
221 \\
122,310 \\
013,131 \\
\overline{2} 12\end{array}$ & $\begin{array}{l}\text { 2. } 213 \\
\text { 2. } 185 \\
\text { 2. } 159 \\
\text { 2. } 089 \\
\text { 2. } 023\end{array}$ & $\begin{array}{r}7 \\
6 \\
3 \\
4 \\
31\end{array}$ & $\begin{array}{c}\overline{3} 41, \frac{421}{431}, \overline{4} 23 \\
340 \\
124,043 \\
134\end{array}$ & $\begin{array}{l}\text { 1. } 425 \\
1.416 \\
1.410 \\
1.391 \\
1.383\end{array}$ & $\begin{array}{l}2 \\
4 \\
3 \\
9 \\
8\end{array}$ \\
\hline $\begin{array}{c}301 \\
\overline{2} 31 \\
103, \overline{1} 32 \\
320 \\
023\end{array}$ & $\begin{array}{l}1.991 \\
1.959 \\
1.942 \\
1.916 \\
1.868\end{array}$ & $\begin{array}{r}3 \\
5 \\
32 \\
14 \\
4\end{array}$ & $\begin{array}{c}332, \overline{5} 11 \\
204,412 \\
414 \\
510 \\
052\end{array}$ & $\begin{array}{l}\text { 1. } 374 \\
\text { 1. } 348 \\
\text { 1. } 337 \\
\text { 1. } 333 \\
1.320\end{array}$ & $\begin{array}{l}9 \\
4 \\
6 \\
3 \\
8\end{array}$ \\
\hline
\end{tabular}




\section{Lanthanum Niobium Titanium Oxide, $\mathrm{LaNbTiO}_{6}$ (monoclinic)}

Powder data cards. None.

Additional published patterns. None.

NBS sample. The sample of lanthanum niobium titanium oxide was prepared at NBS by R. S. Roth from stoichiometric mixtures of the oxides of lanthanum, niobium, and titanium. The sample was first heated at $1300^{\circ} \mathrm{C}$ for $3 \mathrm{hr}$ and then heated at $1350{ }^{\circ} \mathrm{C}$ for $3 \mathrm{hr}$. Spectrographic analysis of the original oxides showed no impurities greater than 0.05 percent.

The sample was colorless. The refractive indices could not be determined because the sample was too fine.

The $d$-values of the three strongest lines are: $3.444,3.331$, and $3.306 \AA$.

Structural data. P. M. de Wolff [1] in 1962 determined that lanthanum niobium titanium oxide has a C-centered monoclinic lattice.

Lattice constants

\begin{tabular}{|c|c|c|c|c|c|}
\hline & & $a$ & $b$ & $c$ & $\beta$ \\
\hline $\begin{array}{l}1962 \\
1963\end{array}$ & $\begin{array}{l}\text { de Wolff }[1] \\
\text { National Bureau of Standards at } \\
\quad 25^{\circ} \mathrm{C} .\end{array}$ & $\begin{array}{l}\stackrel{\circ}{A} \\
\text { 11. } 20 \\
11.196\end{array}$ & $\begin{array}{l}\stackrel{\circ}{A} \\
\text { 8. } 85 \\
8.851\end{array}$ & $\begin{array}{l}\stackrel{\circ}{A} \\
\text { 5. } 27 \\
\text { 5. } 265\end{array}$ & $\begin{array}{l}115^{\circ} 18^{\prime} \\
115^{\circ} 16^{\prime}\end{array}$ \\
\hline
\end{tabular}

\begin{tabular}{|c|c|c|}
\hline \multirow[t]{2}{*}{$h k l$} & \multicolumn{2}{|c|}{$\begin{array}{l}\text { Internal Standard, } \\
\text { Tungsten, } a=3.1648 \AA \\
\mathrm{Cu}, 1.5405 \AA \text { at } 25^{\circ} \mathrm{C}\end{array}$} \\
\hline & $d$ & $I$ \\
\hline & $\AA$ & \\
\hline 110 & 6.68 & 22 \\
\hline 200 & 5. 063 & 10 \\
\hline 111 & 4. 523 & 10 \\
\hline 111 & 3. 444 & 100 \\
\hline 220 & 3. 331 & 100 \\
\hline$\overline{3} 11$ & 3. 306 & 94 \\
\hline 021 & 3. 241 & 36 \\
\hline$\overline{2} 21$ & 3. 183 & 16 \\
\hline 310 & 3. 153 & 10 \\
\hline 130 & 2. 833 & 11 \\
\hline$\overline{2} 02$ & 2. 632 & 31 \\
\hline 131 & 2.573 & 30 \\
\hline 400 & 2. 531 & 20 \\
\hline$\frac{3}{4} 12$ & 2. 421 & 6 \\
\hline & 2. 800 & \\
\hline$\overline{3} 31$ & 2. 273 & 14 \\
\hline 311 & 2. 249 & 4 \\
\hline 330 & 2. 223 & 10 \\
\hline 511 & 2. 170 & 6 \\
\hline 022 & 2. 096 & 9 \\
\hline 112 & 2. 065 & 12 \\
\hline$\overline{4} 22$ & 2. 033 & 16 \\
\hline 041 & 2. 006 & 10 \\
\hline 241 & 1. 993 & 8 \\
\hline 132 & 1. 941 & 8 \\
\hline
\end{tabular}

\begin{tabular}{|c|c|c|}
\hline \multirow[t]{2}{*}{$h k l$} & \multicolumn{2}{|c|}{$\begin{array}{c}\text { Internal Standard, } \\
\text { Tungsten, } a=3.1648 \AA \\
\text { Cu } 1.5405 \AA \text { at } 25^{\circ} \mathrm{C}\end{array}$} \\
\hline & $d$ & $I$ \\
\hline $\begin{array}{l}\overline{3} 32 \\
202 \\
331 \\
531 \\
241\end{array}$ & $\begin{array}{l}\text { 1. } 916 \\
\text { 1. } 868 \\
\text { 1. } 825 \\
\text { 1. } 783 \\
\text { 1. } 761\end{array}$ & $\begin{array}{r}4 \\
10 \\
10 \\
31 \\
16\end{array}$ \\
\hline $\begin{array}{l}150 \\
441 \\
222 \\
621 \\
151\end{array}$ & $\begin{array}{l}\text { 1. } 744 \\
\text { 1. } 731 \\
\text { 1. } 721 \\
\text { 1. } 715 \\
\text { 1. } 678\end{array}$ & $\begin{array}{r}10 \\
9 \\
10 \\
8 \\
6\end{array}$ \\
\hline $\begin{array}{r}530 \\
042, \frac{1}{2} 23 \\
511 \\
620\end{array}$ & $\begin{array}{l}\text { 1. } 669 \\
\text { 1. } 651 \\
\text { 1. } 620 \\
\text { 1. } 603 \\
\text { 1. } 579\end{array}$ & $\begin{array}{r}9 \\
6 \\
10 \\
11 \\
4\end{array}$ \\
\hline $\begin{array}{l}\overline{7} 11 \\
\overline{3} 333 \\
\overline{1} 52 \\
531\end{array}$ & $\begin{array}{l}\text { 1. } 563 \\
\text { 1. } 507 \\
\text { 1. } 459 \\
\text { 1. } 427\end{array}$ & $\begin{array}{l}5 \\
9 \\
4 \\
4\end{array}$ \\
\hline
\end{tabular}

\section{Reference}

[1] P. M. de Wolff, private communication. 


\section{Lithium Phosphate, low form (lithiophospiate), $\mathrm{Li}_{3} \mathrm{PO}_{4}$ (orthorhombic)}

Powder data cards

\begin{tabular}{|c|c|c|}
\hline $\begin{array}{c}\text { Card } \\
\text { number }\end{array}$ & $\begin{array}{c}\text { Index } \\
\text { lines }\end{array}$ & Source \\
\hline $12-230$ & $\begin{array}{r}3.98 \\
3.80 \\
2.67\end{array}$ & Fisher [1] 1958. \\
& \\
\hline
\end{tabular}

A high form is obtained from samples that have been heated above approximately $500^{\circ} \mathrm{C}$.

Additional published pattern. Matias and Bondareva [2] 1957.

NBS sample. The sample of lithium phosphate was obtained from the City Chemical Co., New York, N.Y. Spectrographic analysis showed the following major impurities: 0.1 to 1.0 percent calcium; 0.01 to 0.1 percent each of aluminum, sodium, and strontium; and 0.001 to 0.01 percent each of barium, iron, magnesium, and silicon.

The sample was colorless. The indices of refraction could not be determined because the sample was too fine.

The $d$-values of the three strongest lines are: $3.973,3.797$, and $2.640 \AA$.

Structural data. Zemann [3] in 1960 studied a crystal of $\mathrm{Li}_{3} \mathrm{PO}_{4}$ that had been grown in molten $\mathrm{LiCl}$ and determined that it has the space group $\mathrm{D}_{2_{\mathrm{h}}}^{16}-\mathrm{Pmnb}$ (No. 62) and $4\left(\mathrm{Li}_{3} \mathrm{PO}_{4}\right)$ per unit cell. We find that the lower form apparently has the same structure with only slight changes in the lattice constants, principally in the c-direction. The cell constants of Zambonini and Laves have been converted from $\mathrm{kX}$ to angstrom units for comparison with the NBS values.

Lattice constants

\begin{tabular}{|c|c|c|c|c|}
\hline & & $a$ & $b$ & $c$ \\
\cline { 2 - 5 } 1932 & $\AA$ & $\AA$ & $\AA$ \\
1963 & $\begin{array}{l}\text { Zambonini and } \\
\text { Laves [4]. } \\
\text { National Bureau } \\
\text { of Standards } \\
\text { at 25 }{ }^{\circ} \mathrm{C} .\end{array}$ & 6.08 & 10.28 & 4.87 \\
\pm .0004 & \pm .001 & $\begin{array}{c}4.8452 \\
\pm .0005\end{array}$ \\
\hline
\end{tabular}

\section{References}

[1] D. J. Fisher, Note on lithiophosphate, Am. Mineralogist 43, 761-2 (1958).

[2] V. V. Matias and A. M. Bondareva, Lithiophosphate, a new mineral, Doklady Akad. Nauk S.S.S.R. 112, 124-6 (1957); an English abstract exists in Am. Mineralogist 42, 585 (1957).

[3] J. Zemann, Die Kristallstrukturvon Lithiumphosphat, $\mathrm{Li}_{3} \mathrm{PO}_{4}$, Acta Cryst. 13, 863-7 (1960).

[4] F. Zambonini and F. Laves, UUber die Kristallstruktur des $\mathrm{Li}_{3} \mathrm{PO}_{4}$ und seine Beziehung Zum Strukturtyp des Olivin, Z. Krist. 83, 26-28 (1932).
The density of lithium phosphate, low form, calculated from the NBS lattice constants is 2.480 $\mathrm{g} / \mathrm{cm}^{3}$ at $25^{\circ} \mathrm{C}$.

\begin{tabular}{|c|c|c|}
\hline \multirow[t]{2}{*}{$h k l$} & \multicolumn{2}{|c|}{$\begin{array}{c}\text { Internal Standard } \\
\text { Silver, } a=4.0861 \AA \\
\mathrm{Cu}, 1.5405 \AA \text { at } 25^{\circ} \mathrm{C}\end{array}$} \\
\hline & $d$ & $I$ \\
\hline $\begin{array}{l}020 \\
120 \\
101 \\
021 \\
121 \\
200\end{array}$ & $\begin{array}{c}\stackrel{\AA}{A} \\
\text { 5. } 232 \\
\text { 3. } 973 \\
\text { 3. } 797 \\
\text { 3. } 554 \\
\text { 3. } 071 \\
\text { 3. } 059\end{array}$ & $\begin{array}{r}34 \\
100 \\
98 \\
56 \\
26\end{array}$ \\
\hline $\begin{array}{l}220 \\
040 \\
002 \\
221 \\
041\end{array}$ & $\begin{array}{l}\text { 2. } 640 \\
\text { 2. } 616 \\
\text { 2. } 423 \\
\text { 2. } 318 \\
\text { 2. } 303\end{array}$ & $\begin{array}{l}64 \\
36 \\
47 \\
31\end{array}$ \\
\hline $\begin{array}{c}022 \\
141 \\
122 \\
320,202 \\
301\end{array}$ & $\begin{array}{l}\text { 2. } 199 \\
\text { 2. } 155 \\
\text { 2. } 070 \\
\text { 1. } 899 \\
\text { 1. } 879\end{array}$ & $\begin{array}{r}<1 \\
4 \\
3 \\
1 \\
2\end{array}$ \\
\hline $\begin{array}{l}241 \\
222 \\
321 \\
142 \\
160\end{array}$ & $\begin{array}{l}\text { 1. } 839 \\
\text { 1. } 785 \\
\text { 1. } 769 \\
\text { 1. } 7074 \\
\text { 1. } 6777\end{array}$ & $\begin{array}{r}4 \\
14 \\
9 \\
1 \\
2\end{array}$ \\
\hline $\begin{array}{c}061 \\
340 \\
161 \\
103 \\
023,312\end{array}$ & $\begin{array}{l}\text { 1. } 6415 \\
\text { 1. } 6083 \\
\text { 1. } 5855 \\
\text { 1. } 5616 \\
\text { 1. } 5431\end{array}$ & $\begin{array}{l}1 \\
3 \\
2 \\
4 \\
3\end{array}$ \\
\hline $\begin{array}{l}400 \\
260 \\
123 \\
420 \\
421\end{array}$ & $\begin{array}{l}\text { 1. } 5287 \\
\text { 1. } 5152 \\
\text { 1. } 4959 \\
\text { 1. } 4675 \\
\text { 1. } 4043\end{array}$ & $\begin{array}{r}11 \\
16 \\
1 \\
1 \\
2\end{array}$ \\
\hline $\begin{array}{l}223 \\
143 \\
440 \\
080 \\
402\end{array}$ & $\begin{array}{l}\text { 1. } 3776 \\
\text { 1. } 3409 \\
\text { 1. } 3203 \\
\text { 1. } 3078 \\
\text { 1. } 2931\end{array}$ & $\begin{array}{r}14 \\
2 \\
2 \\
<1 \\
4\end{array}$ \\
\hline $\begin{array}{c}262 \\
053,361 \\
303 \\
422 \\
243\end{array}$ & $\begin{array}{l}\text { 1. } 2848 \\
\text { 1. } 2788 \\
\text { 1. } 2656 \\
\text { 1. } 2550 \\
\text { 1. } 2533\end{array}$ & $\begin{array}{l}8 \\
4 \\
2 \\
1 \\
1\end{array}$ \\
\hline $\begin{array}{l}280 \\
520 \\
501 \\
281 \\
442\end{array}$ & $\begin{array}{l}\text { 1. } 2027 \\
\text { 1. } 1909 \\
\text { 1. } 1860 \\
\text { 1. } 1676 \\
\text { 1. } 1591\end{array}$ & $\begin{array}{r}<1 \\
2 \\
2 \\
3 \\
2\end{array}$ \\
\hline $\begin{array}{l}460 \\
343 \\
380\end{array}$ & $\begin{array}{l}\text { 1. } 1498 \\
\text { 1. } 1394 \\
\text { 1. } 1014\end{array}$ & $\sum_{1}^{1}$ \\
\hline
\end{tabular}




\section{Lithium Phosphate, high form, $\mathrm{Li}_{3} \mathrm{PO}_{4}$ (orthorhombic)}

Powder data cards. None. Powder data card No. 12-230 has a pattern by Fisher [1] which seems to be for the low form. The sample is changed to the high form when it has been heated above approximately $500^{\circ} \mathrm{C}$.

Additional published patterns. Tien and Hummel [2], 1961.

NBS sample. The sample of lithium phosphate was obtained from the City Chemical Co, New York, N.Y. It was heated at $800^{\circ} \mathrm{C}$ for $15 \mathrm{~min}$.
Spectrographic analysis showed the following major impurities 0.1 to 1.0 percent calcium; 0.01 to 0.1 percent each of aluminum, sodium, and strontium; and 0.001 to 0.01 percent each of barium, iron, magnesium, and silicon.

The sample was colorless and optically positive, with the indices of refraction $\mathrm{N}_{\alpha}=1.550, \mathrm{~N}_{\beta}=$ 1.556 , and $\mathrm{N}_{\gamma}=1.560$.

The $d$-values of the three strongest lines are: $3.978,3.834$ and $2.640 \AA$.

\begin{tabular}{|c|c|c|c|c|c|}
\hline \multirow[t]{2}{*}{$h l k l$} & \multicolumn{2}{|c|}{$\begin{array}{l}\text { Internal Standard, } \\
\text { Tungsten, } a \doteq 3.1648 \AA \\
\mathrm{Cu} 1.5405 \AA \text { at } 25{ }^{\circ} \mathrm{C}\end{array}$} & \multirow[t]{2}{*}{$h k l$} & \multicolumn{2}{|c|}{$\begin{array}{c}\text { Internal Standard, } \\
\text { Tungsten, } a=3.1648 \AA \\
\mathrm{Cu} 1.5405 \AA \text { at } 25^{\circ} \mathrm{C}\end{array}$} \\
\hline & $d$ & $I$ & & $d$ & $I$ \\
\hline $\begin{array}{c}020 \\
011 \\
120 \\
101 \\
021,111\end{array}$ & \begin{tabular}{l}
\multicolumn{1}{c}{$\AA$} \\
5. 24 \\
4. 46 \\
3. 978 \\
3. 834 \\
3. 588
\end{tabular} & $\begin{array}{c}29 \\
7 \\
100 \\
93 \\
63\end{array}$ & $\begin{array}{l}171 \\
162 \\
143 \\
431 \\
233\end{array}$ & 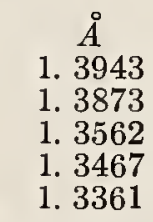 & $\begin{array}{l}\sum_{1} \\
\sum 1 \\
<1 \\
2\end{array}$ \\
\hline $\begin{array}{l}121 \\
200 \\
220 \\
040 \\
131\end{array}$ & $\begin{array}{l}\text { 3. } 093 \\
3.058 \\
2.640 \\
2.619 \\
2.583\end{array}$ & $\begin{array}{l}18 \\
17 \\
67 \\
37 \\
27\end{array}$ & $\begin{array}{c}440 \\
080 \\
402 \\
262 \\
180,361\end{array}$ & $\begin{array}{l}\text { 1. } 3198 \\
\text { 1. } 3095 \\
\text { 1. } 2984 \\
\text { 1. } 2910 \\
1.2806\end{array}$ & $\begin{array}{r}1 \\
<1 \\
5 \\
8 \\
5\end{array}$ \\
\hline $\begin{array}{l}311 \\
002 \\
140 \\
221 \\
041\end{array}$ & $\begin{array}{l}\text { 2. } 521 \\
2.462 \\
2.406 \\
2.327 \\
2.313\end{array}$ & $\begin{array}{r}14 \\
47 \\
3 \\
3 \\
4\end{array}$ & $\begin{array}{l}313 \\
081 \\
323 \\
181 \\
333\end{array}$ & $\begin{array}{l}\text { 1. } 2682 \\
\text { 1. } 2651 \\
\text { 1. } 2414 \\
\text { 1. } 2390 \\
1.2002\end{array}$ & $\begin{array}{r}4 \\
2 \\
<1 \\
<1 \\
4\end{array}$ \\
\hline $\begin{array}{l}141 \\
122 \\
231 \\
051 \\
212\end{array}$ & $\begin{array}{l}\text { 2. } 163 \\
2.095 \\
2.084 \\
1.927 \\
1.885\end{array}$ & $\begin{array}{r}2 \\
6 \\
5 \\
3 \\
<1\end{array}$ & $\begin{array}{c}520,253 \\
501 \\
163 \\
281 \\
034\end{array}$ & $\begin{array}{l}\text { 1. } 1905 \\
\text { 1. } 1875 \\
\text { 1. } 1731 \\
\text { 1. } 1688 \\
1.1608\end{array}$ & $\begin{array}{l}3 \\
2 \\
2 \\
3 \\
1\end{array}$ \\
\hline $\begin{array}{l}311 \\
241 \\
151 \\
222 \\
042\end{array}$ & $\begin{array}{l}1.853 \\
\text { 1. } 844 \\
1.838 \\
1.800 \\
1.793\end{array}$ & $\begin{array}{r}5 \\
4 \\
4 \\
14 \\
5\end{array}$ & $\begin{array}{c}460 \\
182,214 \\
531 \\
380 \\
234\end{array}$ & $\begin{array}{l}\text { 1. } 1499 \\
\text { 1. } 1354 \\
\text { 1. } 1231 \\
\text { 1. } 1015 \\
1.0850\end{array}$ & $\begin{array}{l}<1 \\
\sum 1 \\
<1 \\
2 \\
1\end{array}$ \\
\hline $\begin{array}{l}321 \\
060 \\
160 \\
331 \\
061\end{array}$ & $\begin{array}{l}\text { 1. } 772 \\
\text { 1. } 745 \\
\text { 1. } 679 \\
\text { 1. } 657 \\
\text { 1. } 646\end{array}$ & $\begin{array}{r}6 \\
<1 \\
3 \\
4 \\
2\end{array}$ & $\begin{array}{c}522 \\
314 \\
154 \\
363 \\
064,382\end{array}$ & $\begin{array}{l}\text { 1. } 0719 \\
\text { 1. } 0481 \\
\text { 1. } 0456 \\
\text { 1. } 0314 \\
\text { 1. } 0056\end{array}$ & $\begin{array}{l}<1 \\
1 \\
<1 \\
<1 \\
1\end{array}$ \\
\hline $\begin{array}{c}251 \\
340 \\
103 \\
023 \\
341,400\end{array}$ & $\begin{array}{l}1.6305 \\
1.6080 \\
1.5848 \\
1.5659 \\
1.5285\end{array}$ & $\begin{array}{l}1 \\
3 \\
4 \\
5 \\
9\end{array}$ & \begin{tabular}{|c|}
$2 \cdot 10 \cdot 0$ \\
391 \\
453 \\
$472,503,621$ \\
481
\end{tabular} & $\begin{array}{r}0.9910 \\
.9902 \\
.9867 \\
.9805 \\
.9749\end{array}$ & $\begin{array}{l}\sum 1 \\
\sum 1 \\
\sum 1\end{array}$ \\
\hline $\begin{aligned} 260 \\
420 \\
133 \\
213,332,071 \\
421\end{aligned}$ & $\begin{array}{l}\text { 1. } 5158 \\
\text { 1. } 4673 \\
\text { 1. } 4429 \\
\text { 1. } 4322 \\
1.4067\end{array}$ & $\begin{array}{r}17 \\
1 \\
7 \\
5 \\
<1\end{array}$ & $\begin{array}{c}283,552 \\
533 \\
602,354\end{array}$ & $\begin{array}{l}.9705 \\
.9442 \\
.9414\end{array}$ & $\sum_{1}$ \\
\hline
\end{tabular}




\section{Lithium Phosphate, high form, $\mathrm{Li}_{3} \mathrm{PO}_{4}$ (orthorhombic)-Continued}

Structural data. Zemann [3] in 1960 studied a crystal of $\mathrm{Li}_{3} \mathrm{PO}_{4}$ that had been grown in molten $\mathrm{LiCl}$ and determined that it has the space group $\mathrm{D}_{2 \mathrm{~h}}^{16}-\mathrm{Pmnb}($ No. 62$)$ and $4\left(\mathrm{Li}_{3} \mathrm{PO}_{4}\right)$ per unit cell. The cell constants of Zambonini and Laves have been converted from $\mathrm{kX}$ to angstrom units for comparison with the NBS values.

Lattice constants

\begin{tabular}{|c|c|c|c|c|}
\hline \multirow{5}{*}{$\begin{array}{l}1932 \\
1960 \\
1963\end{array}$} & \multirow{5}{*}{$\begin{array}{l}\text { Zambonini and } \\
\text { Laves [4]. } \\
\text { Zemann [3] } \\
\text { National Bureau } \\
\text { of Standards } \\
\text { at } 25^{\circ} \mathrm{C} \text {. }\end{array}$} & $a$ & $b$ & $c$ \\
\hline & & $\stackrel{\circ}{A}$ & $\AA$ & $\stackrel{\circ}{A}$ \\
\hline & & 6. 08 & 10. 28 & 4. 87 \\
\hline & & 6.12 & 10. 53 & 4. 93 \\
\hline & & $\begin{array}{r}6.1147 \\
\pm .0005\end{array}$ & $\begin{array}{r}10.475 \\
\pm .001\end{array}$ & $\begin{array}{r}4.9228 \\
\pm .0005\end{array}$ \\
\hline
\end{tabular}

The density of lithium phosphate, high form, calculated from the NBS lattice constants is 2.439 $\mathrm{g} / \mathrm{cm}^{3}$ at $25^{\circ} \mathrm{C}$.

\section{References}

[1] D. J. Fisher, Note on lithiophosphate, Am. Mineralogist 43, 761-2 (1958).

[2] T. Y. Tien and F. A. Hummel, Studies in lithium oxide systems: X, lithium phosphate compounds, J. Am. Ceram. Soc. 44, 206-8 (1961).

[3] J. Zemann, Die Kristallstruktur von Lithiumphosphat, $\mathrm{Li}_{3} \mathrm{PO}_{4}$, Acta Cryst. 13, 863-7 (1960).

[4] F. Zambonini and F. Laves, Úber die Kristallstruktur des $\mathrm{Li}_{3} \mathrm{PO}_{4}$ und seine Beziehung Zum Strukturtyp des Olivin, Z. Krist. 83, 26-28 (1932). 
Magnesium Ammonium Phosphate Hexahydrate (struvite), $\mathrm{MgNH}_{4} \mathrm{PO}_{4} \cdot 6 \mathrm{H}_{2} \mathrm{O}$ (orthorhombic)

Powder data cards

\begin{tabular}{|c|c|c|}
\hline $\begin{array}{c}\text { Card } \\
\text { number }\end{array}$ & $\begin{array}{c}\text { Index } \\
\text { lines }\end{array}$ & Source \\
\cline { 2 - 3 } $5-0316$ & $\begin{array}{r}4.28 \\
2.93 \\
2.69\end{array}$ & $\begin{array}{l}\text { Hanawalt, Rinn and Frevel [1] } \\
1938 .\end{array}$ \\
\hline
\end{tabular}

Additional published patterns. None.

NBS sample. The sample of struvite was precipitated at NBS from a solution of magnesium sulfate by adding a solution of ammonium monohydrogen orthophosphate. Spectrographic analysis showed the following major impurities: 0.01 to 0.1 percent sodium, and 0.001 to 0.01 percent each of calcium, iron, and silicon.

The sample was colorless and optically positive. The indices of refraction are $\mathrm{N}_{\alpha}=1.493, \mathrm{~N}_{\beta}=$ 1.496 , and $\mathrm{N}_{\gamma}=1.501$.

The $d$-values of the three strongest lines are: $4.257,5.601$, and $2.919 \AA$.

Structural data. Bland and Basinski [2] in 1959 determined that struvite has the space group $\mathrm{C}_{2 \mathrm{v}}^{7}-\mathrm{Pm} 2 \mathrm{n}(\mathrm{No} .31)$ and $2\left(\mathrm{MgNH}_{4} \mathrm{PO}_{4} \cdot 6 \mathrm{H}_{2} \mathrm{O}\right)$ per unit cell.

\section{Lattice constants}

\begin{tabular}{|c|c|c|c|c|}
\hline \multirow{5}{*}{$\begin{array}{l}1944 \\
1959 \\
1963\end{array}$} & \multirow{5}{*}{$\begin{array}{l}\text { Palache, Berman } \\
\text { and Frondel [3] } \\
\text { Bland and Basin- } \\
\text { ski [2] } \\
\text { National Bureau } \\
\text { of Standards at } \\
25^{\circ} \mathrm{C}\end{array}$} & $a$ & $b$ & $c$ \\
\hline & & $\stackrel{\circ}{A}$ & $\AA$ & $\AA$ \\
\hline & & 6.10 & 11.20 & 0.97 \\
\hline & & 6. 13 & 11. 19 & 6. 92 \\
\hline & & $\begin{array}{r}6.945 \\
\pm .001\end{array}$ & $\begin{array}{l}11.208 \\
\pm .002\end{array}$ & $\begin{array}{r}6.1355 \\
\pm .0008\end{array}$ \\
\hline
\end{tabular}

The density calculated from the NBS lattice constants is $1.706 \mathrm{~g} / \mathrm{cm}^{3}$ at $25^{\circ} \mathrm{C}$.

\section{References}

[1] J. D. Hanawalt, H. W. Rinn, and L. K. Frevel, Chemical analysis by x-ray diffraction, Ind. Eng. Chem., Anal. Ed. 10, 457-512 (1938).

[2] J. A. Bland and S. J. Basinski, Crystal symmetry of struvite (guanite), Nature 183, 1385-7 (1959).

[3] C. Palache, H. Berman, and C. Frondel, Dana's System of Mineralogy, 7th Ed. 2, 715 (1944).

\begin{tabular}{|c|c|c|}
\hline \multirow[t]{2}{*}{$h k l$} & \multicolumn{2}{|c|}{$\begin{array}{l}\text { Internal Standard, } \\
\text { Tungsten, } a=3.1648 \AA \\
\mathrm{Cu}, 1.5405 \AA \text { at } 25^{\circ} \mathrm{C}\end{array}$} \\
\hline & $d$ & $I$ \\
\hline $\begin{array}{l}001 \\
110 \\
020 \\
011 \\
101\end{array}$ & \begin{tabular}{l}
\multicolumn{1}{c}{} \\
6. 14 \\
5. 905 \\
5. 601 \\
5. 378 \\
4. 600
\end{tabular} & $\begin{array}{r}7 \\
41 \\
58 \\
27 \\
6\end{array}$ \\
\hline $\begin{array}{l}111 \\
021 \\
121 \\
200 \\
130\end{array}$ & $\begin{array}{l}\text { 4. } 257 \\
\text { 4. } 139 \\
\text { 3. } 557 \\
\text { 3. } 475 \\
\text { 3. } 289\end{array}$ & $\begin{array}{r}100 \\
40 \\
4 \\
11 \\
27\end{array}$ \\
\hline $\begin{array}{l}031 \\
002 \\
201 \\
012 \\
211\end{array}$ & $\begin{array}{l}\text { 3. } 192 \\
\text { 3. } 067 \\
\text { 3. } 022 \\
\text { 2. } 958 \\
\text { 2. } 919\end{array}$ & $\begin{array}{r}2 \\
3 \\
14 \\
23 \\
54\end{array}$ \\
\hline $\begin{array}{l}040 \\
112 \\
022 \\
221 \\
041\end{array}$ & $\begin{array}{l}\text { 2. } 802 \\
\text { 2. } 722 \\
\text { 2. } 690 \\
\text { 2. } 660 \\
\text { 2. } 548\end{array}$ & $\begin{array}{r}34 \\
15 \\
50 \\
43 \\
3\end{array}$ \\
\hline $\begin{array}{l}122 \\
141 \\
231 \\
202 \\
212\end{array}$ & $\begin{array}{l}\text { 2. } 511 \\
\text { 2. } 394 \\
2.352 \\
2.300 \\
2.253\end{array}$ & $\begin{array}{r}7 \\
5 \\
12 \\
1 \\
4\end{array}$ \\
\hline $\begin{array}{c}240 \\
301 \\
150 \\
222,311 \\
042\end{array}$ & $\begin{array}{l}\text { 2. } 180 \\
\text { 2. } 167 \\
\text { 2. } 133 \\
\text { 2. } 127 \\
\text { 2. } 069\end{array}$ & $\begin{array}{l}4 \\
3 \\
5 \\
7 \\
6\end{array}$ \\
\hline $\begin{array}{c}241 \\
003 \\
151 \\
142 \\
103,232\end{array}$ & $\begin{array}{l}\text { 2. } 054 \\
2.046 \\
\text { 2. } 014 \\
\text { 1. } 983 \\
1.960\end{array}$ & $\begin{array}{r}11 \\
10 \\
5 \\
14\end{array}$ \\
\hline $\begin{array}{l}113 \\
023 \\
331 \\
123 \\
312\end{array}$ & $\begin{array}{l}\text { 1. } 932 \\
\text { 1. } 921 \\
\text { 1. } 873 \\
\text { 1. } 851 \\
\text { 1. } 822\end{array}$ & $\begin{array}{l}2 \\
3 \\
5 \\
3 \\
3\end{array}$ \\
\hline $\begin{array}{c}052 \\
251 \\
033 \\
203 \\
133,400\end{array}$ & $\begin{array}{l}\text { 1. } 810 \\
\text { 1. } 801 \\
\text { 1. } 794 \\
\text { 1. } 762 \\
\text { 1. } 737\end{array}$ & $\begin{array}{r}8 \\
14 \\
10 \\
9 \\
14\end{array}$ \\
\hline $\begin{array}{l}341 \\
223 \\
332\end{array}$ & $\begin{array}{l}\text { 1. } 714 \\
\text { 1. } 681 \\
\text { 1. } 657\end{array}$ & $\begin{array}{l}5 \\
4 \\
4\end{array}$ \\
\hline
\end{tabular}




\section{Potassium Chlorate, $\mathrm{KClO}_{3}$ (monoclinic)}

Powder Data cards

\begin{tabular}{|c|c|c|}
\hline $\begin{array}{c}\text { Card } \\
\text { number }\end{array}$ & $\begin{array}{l}\text { Index } \\
\text { lines }\end{array}$ & Source \\
\hline $1-0599$ & $\begin{array}{l}3.45 \\
2.79 \\
2.86\end{array}$ & $\begin{array}{l}\text { Hanawalt, Rinn and Frevel } \\
\text { [1] 1938. }\end{array}$ \\
\hline $12-571$ & $\begin{array}{l}\text { 3. } 46 \\
\text { 2. } 88 \\
\text { 2. } 80\end{array}$ & $\begin{array}{l}\text { Institute of Physics, Univer- } \\
\text { sity College, Cardiff. }\end{array}$ \\
\hline
\end{tabular}

Additional published patterns. None.

NBS sample. The sample of potassium chlorate was obtained from the General Chemical Co., New York, N.Y., and recrystallized to sharpen the pattern. Spectrographic analysis showed the following impurities: 0.001 to 0.01 percent each of aluminum, barium, iron, rubidium, silicon, sodium, and strontium.

The color of the sample was white. It is optically negative with the indices of refraction $\mathrm{N}_{\alpha}=1.408, \mathrm{~N}_{\beta}=1.516$, and $\mathrm{N}_{\gamma}=1.524 ; 2 \mathrm{~V}$ was very small.

The $d$-values of the three strongest lines are: $3.45,3.34$, and $2.868 \AA$.

Structural data. Zachariasen [2] and [3] in 1928 determined that potassium chlorate has the space group $\mathrm{C}_{2 \mathrm{~h}}^{2} \mathrm{P}_{1} / \mathrm{m}$ (No. 11) and $2\left[\mathrm{KClO}_{3}\right]$ per unit cell. The unit cell measurements of Ieviñs and Ozols and Zachariasen have been converted from $\mathrm{kX}$ to angstrom units for comparison with the NBS values.

The density of potassium chlorate calculated from the NBS lattice constants is $2.339 \mathrm{~g} / \mathrm{cm}^{3}$ at $26^{\circ} \mathrm{C}$.

\section{References}

[1] J. D. Hanawalt, H. W. Rinn, and L. K. Frevel, Chemical analysis by X-ray diffraction, Ind. Eng. Chem. Anal. Ed. 10, 457-513 (1938).

[2] W. H. Zachariasen, The crystal structure of sesquioxides and compounds of the type $\mathrm{ABO}_{3}$, Skrifter Norske Videnskaps-Akad. Oslo I: Mat.-Naturv. Kl. Nr. 4, 82 (1928).

[3] W. H. Zachariasen, The crystal structure of potassium chlorate, Z. Krist. F1, 501 (1929).

[4] A. Ievinş and J. Ozols, Precision determination of lattice constants of monoclinic crystals, Doklady Akad. Nauk SSSR 91, 527-30 (1953).

\begin{tabular}{|c|c|c|}
\hline \multirow[t]{2}{*}{$h k l$} & \multicolumn{2}{|c|}{$\begin{array}{l}\text { Internal Standard, } \\
\text { Tungsten, } a=3.1648 \AA \\
\mathrm{Cu}, 1.5405 \AA \text { at } 26^{\circ} \mathrm{C}\end{array}$} \\
\hline & $d$ & $I$ \\
\hline $\begin{array}{l}001 \\
101 \\
011 \\
110 \\
002\end{array}$ & 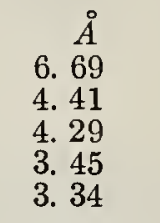 & $\begin{array}{r}3 \\
10 \\
5 \\
100 \\
60\end{array}$ \\
\hline $\begin{array}{l}\overline{1} 02 \\
101 \\
012 \\
020 \\
111\end{array}$ & $\begin{array}{l}\text { 3. } 234 \\
\text { 3. } 204 \\
2.868 \\
2.794 \\
\text { 2. } 779\end{array}$ & $\begin{array}{r}3 \\
4 \\
35 \\
33 \\
23\end{array}$ \\
\hline $\begin{array}{c}021 \\
120 \\
201 \\
102 \\
\overline{2} 11, \overline{1} 13\end{array}$ & $\begin{array}{l}2.579 \\
2.356 \\
2.327 \\
2.309 \\
2.149\end{array}$ & $\begin{array}{r}4 \\
3 \\
18 \\
2 \\
6\end{array}$ \\
\hline $\begin{array}{l}022 \\
112 \\
122 \\
013 \\
203\end{array}$ & $\begin{array}{l}2.145 \\
2.135 \\
2.115 \\
2.070 \\
1.919\end{array}$ & $\begin{array}{r}7 \\
7 \\
17 \\
2 \\
5\end{array}$ \\
\hline $\begin{array}{c}201 \\
\overline{2} 13 \\
211 \\
\overline{2} 21, \overline{1} 23 \\
122\end{array}$ & $\begin{array}{l}\text { 1. } 902 \\
\text { 1. } 815 \\
\text { 1. } 800 \\
\text { 1. } 7888 \\
\text { 1. } 7800\end{array}$ & $\begin{array}{l}5 \\
2 \\
1 \\
2 \\
1\end{array}$ \\
\hline $\begin{array}{l}104 \\
103 \\
023 \\
130 \\
113\end{array}$ & $\begin{array}{l}\text { 1. } 7730 \\
\text { 1. } 7615 \\
\text { 1. } 7421 \\
\text { 1. } 7149 \\
\text { 1. } 6800\end{array}$ & $\begin{array}{r}<1 \\
<1 \\
1 \\
2 \\
1\end{array}$ \\
\hline $\begin{array}{l}032 \\
132 \\
131 \\
014 \\
223\end{array}$ & $\begin{array}{l}1.6279 \\
\text { 1. } 6145 \\
\text { 1. } 6111 \\
\text { 1. } 6014 \\
\text { 1. } 5820\end{array}$ & $\begin{array}{r}5 \\
2 \\
2 \\
3 \\
<1\end{array}$ \\
\hline $\begin{array}{l}221 \\
214 \\
302 \\
124 \\
123\end{array}$ & $\begin{array}{l}1.5722 \\
1.5539 \\
1.5428 \\
1.4973 \\
1.4897\end{array}$ & $\begin{array}{r}<1 \\
<1 \\
<1 \\
4 \\
4\end{array}$ \\
\hline $\begin{array}{l}\overline{3} 11 \\
\overline{3} 03 \\
033 \\
\overline{3} 13 \\
310\end{array}$ & $\begin{array}{l}1.4848 \\
\text { 1. } 4700 \\
\text { 1. } 4296 \\
\text { 1. } 4213 \\
1.4137\end{array}$ & $\begin{array}{r}4 \\
<1 \\
1 \\
2 \\
<1\end{array}$ \\
\hline $\begin{array}{c}040 \\
203 \\
005, \overline{2} 33 \\
\overline{2} 15 \\
213\end{array}$ & $\begin{array}{l}\text { 1. } 3978 \\
\text { 1. } 3517 \\
\text { 1. } 3371 \\
\text { 1. } 3254 \\
\text { 1. } 3136\end{array}$ & $\begin{array}{r}2 \\
<1 \\
1 \\
<1 \\
<1\end{array}$ \\
\hline $\begin{array}{l}\overline{3} 23 \\
042\end{array}$ & $\begin{array}{l}\text { 1. } 3009 \\
\text { 1. } 2899\end{array}$ & $<2$ \\
\hline
\end{tabular}




\section{Potossium Chlorate, $\mathrm{KCIO}_{3}$ (monoclinic)-Continued}

Lattice constants

\begin{tabular}{|c|c|c|c|c|c|}
\hline \multirow{3}{*}{$\begin{array}{l}1929 \\
1953 \\
1963\end{array}$} & \multirow{3}{*}{$\begin{array}{l}\text { Zachariasen [3] } \\
\text { Ievins and Ozols [4] } \\
\text { National Bureau of Standards at } \\
\quad 26^{\circ} \mathrm{C}\end{array}$} & $a$ & $b$ & $c$ & $\beta$ \\
\hline & & $\begin{array}{l}\stackrel{\AA}{A} \\
\text { 4. } 656 \\
4.6569\end{array}$ & $\begin{array}{l}\stackrel{\AA}{A} \\
\text { 5. } 596 \\
\text { 5. } 59089\end{array}$ & $\begin{array}{l}\stackrel{\AA}{A} \\
7.099 \\
7.0991\end{array}$ & $\begin{array}{l}109^{\circ} 38^{\prime} \\
109^{\circ} 38.9^{\prime}\end{array}$ \\
\hline & & $\begin{array}{r}4.6553 \\
\pm .0002\end{array}$ & $\begin{array}{r}5.5905 \\
\pm .0006\end{array}$ & $\begin{array}{r}7.1006 \\
\pm .0005\end{array}$ & $\begin{array}{r}109^{\circ} 41.1^{\prime} \\
\pm .3^{\prime}\end{array}$ \\
\hline
\end{tabular}

\section{Potassium Lithium Sulfate, $\mathrm{KLiSO}_{4}$ (hexagonal)}

Powder data cards. None.

Additional published patterns. None.

NBS sample. The sample of potassium lithium sulfate was prepared at NBS by dissolving stoichiometric amounts of solid potassium hydroxide and lithium hydroxide in a small amount of water. When the mixture was at room temperature a drop of 1 percent bromcresol-purple was added and the mixture neutralized with $\mathrm{H}_{2} \mathrm{SO}_{4}$. The solution was evaporated to dryness and the precipitate was then heated to about $750{ }^{\circ} \mathrm{C}$ to sharpen the pattern. Spectrographic analysis showed the following impurities: 0.01 to 0.1 percent each of calcium, indium, and sodium; and 0.001 to 0.01 percent each of aluminum, rubidium, and silicon.

The sample is colorless and optically negative. The indices of refraction are $\mathrm{N}_{\mathrm{o}}=1.471$ and $\mathrm{N}_{\mathrm{e}}$ $=1.469$.

The $d$-values of the three strongest lines are: $3.960,3.099$, and $2.573 \AA$.

Structural data. Nowacki [1] in 1942 determined that potassium lithium sulfate has the space group $\mathrm{C}_{6}^{6}-\mathrm{P} 6 / 3$ (No. 173 ) and $2\left(\mathrm{KLiSO}_{4}\right)$ per unit cell. The lattice constants of Nowacki have been converted from $\mathrm{kX}$ to angstrom units for comparison with the NBS values.

\section{Lattice constants}

\begin{tabular}{|c|c|c|c|}
\hline & & $a$ & $c$ \\
\hline $\begin{array}{l}1942 \\
1963\end{array}$ & $\begin{array}{l}\text { Nowacki }[1] \\
\text { National Bureau of } \\
\quad \text { Standards at } 26^{\circ} \mathrm{C}\end{array}$ & $\begin{array}{c}\stackrel{\AA}{A} \\
\text { 5. } 14 \\
\text { 5. } 1457\end{array}$ & $\begin{array}{c}\stackrel{\AA}{A} \\
\text { 8. } 62 \\
\text { 8. } 6298\end{array}$ \\
\hline
\end{tabular}

The density of potassium lithium sulfate calculated from NBS lattice constants is 2.385 $\mathrm{g} / \mathrm{cm}^{3}$ at at $26{ }^{\circ} \mathrm{C}$.

\begin{tabular}{|c|c|c|}
\hline \multirow[t]{2}{*}{$h k l$} & \multicolumn{2}{|c|}{$\begin{array}{l}\text { Internal Standard, } \\
\text { Tungsten, } a=3.1648 \AA \\
\mathrm{Cu}, 1.5405 \AA \text { at } 26^{\circ} \mathrm{C}\end{array}$} \\
\hline & $d$ & $I$ \\
\hline $\begin{array}{l}002 \\
101 \\
102 \\
110 \\
111\end{array}$ & $\begin{array}{c}\AA \\
\text { 4. } 320 \\
3.960 \\
\text { 3. } 099 \\
2.573 \\
2.465\end{array}$ & $\begin{array}{r}12 \\
100 \\
70 \\
34 \\
16\end{array}$ \\
\hline $\begin{array}{c}103 \\
200 \\
112 \\
201,004 \\
202\end{array}$ & $\begin{array}{l}\text { 2. } 418 \\
\text { 2. } 225 \\
\text { 2. } 211 \\
\text { 2. } 158 \\
\text { 1. } 981\end{array}$ & $\begin{array}{r}5 \\
8 \\
20 \\
19 \\
12\end{array}$ \\
\hline $\begin{array}{c}113 \\
203 \\
210 \\
114,211 \\
105\end{array}$ & $\begin{array}{l}\text { 1. } 919 \\
\text { 1. } 762 \\
\text { 1. } 683 \\
\text { 1. } 653 \\
\text { 1. } 6096\end{array}$ & $\begin{array}{r}4 \\
2 \\
3 \\
11 \\
5\end{array}$ \\
\hline $\begin{array}{l}212 \\
204 \\
300 \\
213 \\
106\end{array}$ & $\begin{array}{l}\text { 1. } 5690 \\
\text { 1. } 5499 \\
\text { 1. } 4852 \\
\text { 1. } 4533 \\
\text { 1. } 3687\end{array}$ & $\begin{array}{r}11 \\
4 \\
11 \\
2 \\
2\end{array}$ \\
\hline $\begin{array}{l}205 \\
214 \\
220 \\
116 \\
222\end{array}$ & $\begin{array}{l}\text { 1. } 3647 \\
\text { 1. } 3276 \\
\text { 1. } 2864 \\
\text { 1. } 2550 \\
\text { 1. } 2329\end{array}$ & $\begin{array}{l}3 \\
3 \\
5 \\
5 \\
2\end{array}$ \\
\hline $\begin{array}{c}311,304 \\
206 \\
215 \\
107,312 \\
313\end{array}$ & $\begin{array}{l}\text { 1. } 2237 \\
\text { 1. } 2081 \\
\text { 1. } 2055 \\
\text { 1. } 1880 \\
\text { 1. } 1356\end{array}$ & $\begin{array}{l}6 \\
2 \\
3 \\
3 \\
1\end{array}$ \\
\hline $\begin{array}{c}401,224 \\
216 \\
402 \\
108 \\
403 \\
306\end{array}$ & $\begin{array}{l}\text { 1. } 1048 \\
\text { 1. } 0939 \\
\text { 1. } 0787 \\
\text { 1. } 0484 \\
\text { 1. } 0389 \\
\text { 1. } 0335\end{array}$ & $\begin{array}{l}2 \\
1 \\
2 \\
1 \\
1 \\
1\end{array}$ \\
\hline
\end{tabular}

\section{Reference}

[1] W. Nowacki, Beziehungen $z$ wischen $\mathrm{K}_{2}\left[\mathrm{AlSiO}_{4}\right]$ ('TietKaliophilit), $\mathrm{Ba}\left[\mathrm{Al}_{2} \mathrm{O}_{4}\right]$, K[LiSO $\left.{ }_{4}\right], \mathrm{Na}\left[\mathrm{AlSiO}_{4}\right]$ ( $\mathrm{Ne}-$ phelin) und $\mathrm{Si}_{2} \mathrm{O}_{4}$ ( $\beta$-Tridymit), Naturwissenschaften 30, 471-472 (1942). 
Powder data cards. None.

Additional published patterns. Wilson [1] 1941.

NBS sample. The sample of potassium perchromate was obtained from City Chemical Corp., New York, N.Y. Spectrographic analysis did not show any impurities greater than the range 0.0001 to 0.001 percent.

The color of the sample was deep red. The indices of refraction are $\mathrm{N}_{0}=1.774$ and $\mathrm{N}_{\mathrm{e}}=1.730$. It is optically negative.

The $d$-values of the three strongest lines are: $2.979,2.372$, and $1.856 \AA$.

Structural data. Wilson [1] in 1941 determined that potassium perchromate has the space group $\mathrm{D}_{2 \mathrm{~d}}^{11}-\mathrm{I} 42 \mathrm{~m}(\mathrm{No} .121)$ and $2\left(\mathrm{~K}_{3} \mathrm{CrO}_{8}\right)$ per unit cell. Potassium perchromate is used as a structure type. The unit cell measurements reported by Wilson have been converted from $\mathrm{kX}$ to angstrom units for comparison with NBS values.

Lattice constants

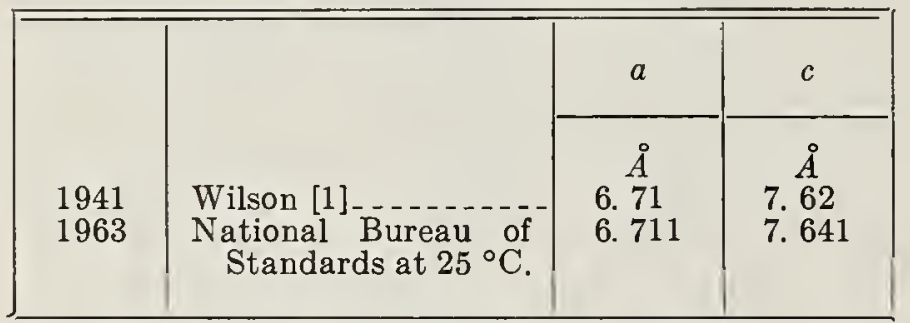

The density of potassium perchromate calculated from NBS lattice constants is $2.869 \mathrm{~g} / \mathrm{cm}^{3}$ at $25^{\circ} \mathrm{C}$.

\section{Reference}

[1] I. A. Wilson, X-ray analysis of potassium perchromate, $\mathrm{K}_{2} \mathrm{CrO}_{8}$ and isomorphous compounds, Arkiv. Kemi Minerali. Geol. B15, 1-7 (1941).

\begin{tabular}{|c|c|c|}
\hline \multirow[t]{2}{*}{$h k l$} & \multicolumn{2}{|c|}{$\begin{array}{l}\text { Internal Standard, } \\
\text { Tungsten, } a=3.1648 \AA \\
\mathrm{Cu}, 1: 5405 \AA \text { at } 25{ }^{\circ} \mathrm{C}\end{array}$} \\
\hline & $d$ & $I$ \\
\hline $\begin{array}{l}101 \\
110 \\
002 \\
200 \\
112\end{array}$ & $\begin{array}{c}\stackrel{\AA}{A} \\
\text { 5. } 054 \\
\text { 4. } 749 \\
\text { 3. } 822 \\
\text { 3. } 357 \\
\text { 2. } 979\end{array}$ & $\begin{array}{r}42 \\
18 \\
1 \\
36 \\
100\end{array}$ \\
\hline $\begin{array}{l}211 \\
202 \\
220 \\
301 \\
310\end{array}$ & $\begin{array}{l}\text { 2. } 795 \\
\text { 2. } 524 \\
\text { 2. } 372 \\
\text { 2. } 147 \\
\text { 2. } 121\end{array}$ & $\begin{array}{r}17 \\
5 \\
53 \\
5 \\
2\end{array}$ \\
\hline $\begin{array}{c}222 \\
004 \\
312 \\
321 \\
303,400\end{array}$ & $\begin{array}{l}2.016 \\
1.911 \\
1.856 \\
1.808 \\
1.677\end{array}$ & $\begin{array}{r}7 \\
9 \\
47 \\
7 \\
5\end{array}$ \\
\hline $\begin{array}{l}204 \\
330 \\
402 \\
420 \\
224\end{array}$ & $\begin{array}{l}1.659 \\
1.5815 \\
1.5365 \\
1.5004 \\
1.4880\end{array}$ & $\begin{array}{r}7 \\
5 \\
3 \\
10 \\
12\end{array}$ \\
\hline $\begin{array}{l}332 \\
314 \\
413 \\
501 \\
404\end{array}$ & $\begin{array}{l}\text { 1. } 4615 \\
\text { 1. } 4198 \\
\text { 1. } 3718 \\
\text { 1. } 3219 \\
\text { 1. } 2607\end{array}$ & $\begin{array}{r}10 \\
<1 \\
2 \\
<1 \\
6\end{array}$ \\
\hline $\begin{array}{c}512 \\
116,521 \\
440 \\
325 \\
600 \\
532 \\
316\end{array}$ & $\begin{array}{l}\text { 1. } 2445 \\
1.2299 \\
1.1863 \\
1.1808 \\
1.1183 \\
1.1020 \\
1.0920\end{array}$ & $\begin{array}{r}10 \\
5 \\
4 \\
4 \\
2 \\
3 \\
2\end{array}$ \\
\hline
\end{tabular}




\section{Potassium Zinc Decavanadate 16 Hydrate, $\mathrm{K}_{2} \mathrm{Zn}_{2} \mathrm{~V}_{10} \mathrm{O}_{28} \cdot 16 \mathrm{H}_{2} \mathrm{O}$ (triclinic)}

Powder data cards. None.

Additional published patterns. None.

NBS sample. The sample of potassium zinc decavanadate 16 hydrate, was obtained from $\mathrm{H}$. T. Evans, Jr., U.S. Geological Survey, Washington, D.C. It was prepared from a solution of potassium metavanadate and zinc acetate in water with the $p \mathrm{H}$ adjusted between 3 and 4 with acetic acid. Spectrographic analysis showed the following major impurities: 0.1 to 1.0 percent sodium; 0.01 to 0.1 percent silicon, and 0.001 to 0.01 percent each of aluminum, barium, calcium, chromium, iron, magnesium, molybdenum, lead, and rubidium.

The color of the sample was bright orange. The indices of refraction could not be determined because of imperfections in the crystals.

The $d$-values of the three strongest lines are: $8.18,7.40$, and $9.45 \AA$.

Structural data. Evans, Mrose, and Marvin [1], in 1955 determined that potassium zinc decavanadate 16 hydrate, has the space group $\mathrm{C}_{\mathrm{i}}^{1-\mathrm{PI}}$ ( No. 2 ) and $1\left(\mathrm{~K}_{2} \mathrm{Zn}_{2} \mathrm{~V}_{10} \mathrm{O}_{28} \cdot 16 \mathrm{H}_{2} \mathrm{O}\right)$ per unit cell.

The density of potassium zinc decavanadate calculated from the NBS lattice constants is $2.708 \mathrm{~g} / \mathrm{cm}^{3}$ at $25^{\circ} \mathrm{C}$.

\section{Reference}

[1] H. T. Evans, Jr., M. E. Mrose, and R. Marvin, Constitution of the natural and artificial decavanadates, Am. Mineralogist 40, 314 (1955).

\begin{tabular}{|c|c|c|}
\hline \multirow[t]{2}{*}{$h k l$} & \multicolumn{2}{|c|}{$\begin{array}{l}\text { Internal Standard, } \\
\text { Tungsten } a=3.1648^{\circ} \AA \\
\mathrm{Cu}, 1.5405 \AA \text { at } 25^{\circ} \mathrm{C}\end{array}$} \\
\hline & $d$ & $I$ \\
\hline $\begin{array}{l}010 \\
100 \\
110 \\
001 \\
111\end{array}$ & $\begin{array}{c}\AA \\
\text { 10. } 01 \\
9.45 \\
\text { 8. } 61 \\
\text { 8. } 18 \\
7.40\end{array}$ & $\begin{array}{r}23 \\
28 \\
20 \\
100 \\
35\end{array}$ \\
\hline $\begin{array}{l}\overline{1} 01 \\
0 \overline{1} 1 \\
011 \\
101 \\
\overline{2} \frac{1}{1} 1\end{array}$ & $\begin{array}{l}7.17 \\
6.81 \\
5.95 \\
5.52 \\
5.17\end{array}$ & $\begin{array}{l}3 \\
7 \\
5 \\
6 \\
9\end{array}$ \\
\hline $\begin{array}{l}020 \\
200 \\
\overline{2} 01 \\
1 \overline{1} 1 \\
\overline{2} 21\end{array}$ & $\begin{array}{l}5.00 \\
4.72 \\
4.63 \\
4.60 \\
4.53\end{array}$ & $\begin{array}{r}14 \\
9 \\
4 \\
4 \\
<1\end{array}$ \\
\hline $\begin{array}{l}220 \\
102 \\
002 \\
121 \\
211\end{array}$ & $\begin{array}{l}\text { 4. } 33 \\
\text { 4. } 166 \\
\text { 4. } 094 \\
\text { 3. } 960 \\
\text { 3. } 759\end{array}$ & $\begin{array}{r}<1 \\
3 \\
16 \\
3 \\
4\end{array}$ \\
\hline $\begin{array}{c}201, \overline{222} \\
\overline{1} \overline{3} 1 \\
\overline{2} 02 \\
\overline{2} \overline{3} 1 \\
\overline{3} \overline{2} 1\end{array}$ & $\begin{array}{l}\text { 3. } 697 \\
\text { 3. } 630 \\
\text { 3. } 580 \\
\text { 3. } 548 \\
\text { 3. } 487\end{array}$ & $\begin{array}{l}7 \\
2 \\
3 \\
4 \\
8\end{array}$ \\
\hline $\begin{array}{l}102 \\
0 \overline{2} 2 \\
230 \\
030 \\
112\end{array}$ & $\begin{array}{l}\text { 3. } 448 \\
\text { 3. } 402 \\
\text { 3. } 373 \\
\text { 3. } 333 \\
\text { 3. } 279\end{array}$ & $\begin{array}{l}6 \\
3 \\
1 \\
4 \\
2\end{array}$ \\
\hline $\begin{array}{c}\overline{3} 01 \\
\overline{23} 2,300 \\
\overline{2} 12 \\
022 \\
031\end{array}$ & $\begin{array}{l}\text { 3. } 229 \\
\text { 3. } 149 \\
\text { 3. } 011 \\
\text { 2. } 977 \\
\text { 2. } 951\end{array}$ & $\begin{array}{l}3 \\
5 \\
6 \\
3 \\
5\end{array}$ \\
\hline $\begin{array}{c}\overline{33} 2,330 \\
032, \overline{1} 23 \\
003 \\
\overline{2} 03 \\
\overline{24} 2, \overline{34} 1\end{array}$ & $\begin{array}{l}\text { 2. } 883 \\
\text { 2. } 778 \\
\text { 2. } 730 \\
\text { 2. } 680 \\
\text { 2. } 595\end{array}$ & $\begin{array}{r}3 \\
11 \\
14 \\
1 \\
<1\end{array}$ \\
\hline $\begin{array}{c}\overline{3} \overline{1} 3 \\
222, \overline{1} 13 \\
420 \\
\overline{13} 3, \overline{43} 2 \\
103,331\end{array}$ & $\begin{array}{l}2.569 \\
2.557 \\
2.525 \\
2.485 \\
2.458\end{array}$ & $\begin{array}{l}2 \\
2 \\
2 \\
4 \\
2\end{array}$ \\
\hline $\begin{array}{l}\overline{3} 03 \\
0 \overline{4} 2 \\
\overline{15} 1 \\
\overline{3} 13 \\
\overline{5} \overline{3} 2\end{array}$ & $\begin{array}{l}\text { 2. } 389 \\
\text { 2. } 278 \\
\text { 2. } 164 \\
\text { 2. } 139 \\
\text { 2. } 102\end{array}$ & $\begin{array}{l}9 \\
8 \\
6 \\
8 \\
1\end{array}$ \\
\hline 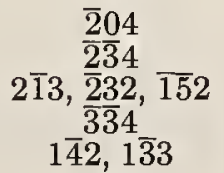 & $\begin{array}{l}\text { 2. } 085 \\
2.071 \\
2.065 \\
2.046 \\
2.006\end{array}$ & $\begin{array}{r}2 \\
1 \\
<1 \\
1 \\
4\end{array}$ \\
\hline $\begin{array}{c}530 \\
0 \overline{4} 3,033 \\
4 \overline{1} 1 \\
\overline{1} 33\end{array}$ & $\begin{array}{l}\text { 1. } 987 \\
\text { 1. } 985 \\
\text { 1. } 964 \\
\text { 1. } 930\end{array}$ & $\begin{array}{r}4 \\
4 \\
1 \\
<1\end{array}$ \\
\hline
\end{tabular}


Potassium Zinc Decavanadete 16 Hydrate, $\mathrm{K}_{2} \mathrm{Zn}_{2} \mathrm{~V}_{10} \mathrm{O}_{28} \cdot 16 \mathrm{H}_{2} \mathrm{O}$ (triclinic)-Continued

Lattice constants

\begin{tabular}{|c|c|c|c|c|c|c|c|}
\hline & & $a$ & $b$ & $c$ & $a$ & $\beta$ & $\gamma$ \\
\hline 1955 & $\begin{array}{l}\text { Evans, Mrose and Mar- } \\
\text { vin [1]. }\end{array}$ & $\stackrel{\AA}{\AA}$ & $\stackrel{A}{A}$ & $\begin{array}{l}\AA \\
8.77\end{array}$ & $104^{\circ} 50^{\prime}$ & $109^{\circ} 29^{\prime}$ & $65^{\circ} 05^{\prime}$ \\
\hline 1963 & $\begin{array}{l}\text { National Bureau of Stand- } \\
\text { ards at } 25^{\circ} \mathrm{C} \text {. }\end{array}$ & $\begin{array}{l}10.778 \\
\pm .003\end{array}$ & $\begin{array}{l}\text { 11. } 146 \\
\pm .003\end{array}$ & $\begin{array}{r}8.774 \\
\pm .003\end{array}$ & $\begin{array}{l}104^{\circ} 57^{\prime} \\
\quad \pm 1^{\prime}\end{array}$ & $\begin{array}{l}109^{\circ} 32^{\prime} \\
\quad \pm 1^{\prime}\end{array}$ & $\begin{array}{r}65^{\circ} 0^{\prime} \\
\pm 2^{\prime}\end{array}$ \\
\hline
\end{tabular}

\section{Rubidium Chromate, $\mathrm{Rb}_{2} \mathrm{CrO}_{4}$ (orthorhombic)}

Powder data cards. None.

Additional published patterns. None.

NBS sample. The sample of rubidium chromate was obtained from the Fairmount Chemical Co., Inc., Newark, N.J. Spectrographic analysis showed the following major impurities: 0.1 to 1.0 percent potassium; 0.01 to 0.1 percent sodium; and 0.001 to 0.01 percent each of aluminum, barium, calcium, and silicon.

The color of the sample was yellow and it is optically positive. The refractive indices are $\mathrm{N}_{\alpha}=1.715, \mathrm{~N}_{\beta}=1.725$, and $\mathrm{N}_{\gamma}=1.759$.

The $d$-values of the three strongest lines are: $3.207,3.191$, and $3.083 \AA$.

Structural data. Smith and Colby [1] in 1940 determined that rubidium chromate has the potassium sulfate structure and the space group $\mathrm{D}_{2 \mathrm{~h}}^{16}$ - Pnam (No. 62) with $4\left(\mathrm{Rb}_{2} \mathrm{CrO}_{4}\right)$ per unit cell.

The lattice constants reported by Smith and Colby have been converted from $\mathrm{kX}$ to angstrom units for comparison with NBS values.

Lattice constants

\begin{tabular}{|c|c|c|c|c|}
\hline & & $a$ & $b$ & $c$ \\
\hline 1940 & $\begin{array}{l}\text { Smith and } \\
\text { Colby }[1]\end{array}$ & $\begin{array}{c}\stackrel{\circ}{A} \\
7.999\end{array}$ & 10. $\stackrel{\AA}{\AA} 726$ & $\begin{array}{c}\stackrel{\circ}{A} \\
\text { 6. } 301\end{array}$ \\
\hline 1963 & $\begin{array}{l}\text { National Bureau } \\
\text { of Standards } \\
\text { at } 25^{\circ} \mathrm{C} \text {. }\end{array}$ & $\begin{array}{r}8.001 \\
\pm .001\end{array}$ & $\begin{array}{l}10.722 \\
\pm .001\end{array}$ & $\begin{array}{r}6.074 \\
\pm .001\end{array}$ \\
\hline
\end{tabular}

The density of rubidium chromate calculated from NBS lattice constants is $3.657 \mathrm{~g} / \mathrm{cm}^{3}$ at $25^{\circ} \mathrm{C}$.

\section{Reference}

[1] H. W. Smith, Jr. and M. Y. Colby, The crystal structure of rubidium chromate, $\mathrm{Rb}_{2} \mathrm{CrO}_{4}, Z$. Krist. 103, 90-95 (1940).

\begin{tabular}{|c|c|c|}
\hline \multirow[t]{2}{*}{$h k l$} & \multicolumn{2}{|c|}{$\begin{array}{l}\text { Internal Standard, } \\
\text { Tungsten } a=3.1648 \AA \\
\mathrm{Cu}, 1.5405 \AA \text { at } 25^{\circ} \mathrm{C}\end{array}$} \\
\hline & $d$ & $I$ \\
\hline $\begin{array}{l}020 \\
120 \\
200 \\
210 \\
121\end{array}$ & $\begin{array}{c}\stackrel{\AA}{A} \\
\text { 5. } 365 \\
\text { 4. } 451 \\
\text { 4. } 004 \\
\text { 3. } 749 \\
\text { 3. } 593\end{array}$ & $\begin{array}{r}1 \\
2 \\
6 \\
14 \\
36\end{array}$ \\
\hline $\begin{array}{l}201 \\
130 \\
220 \\
211 \\
031\end{array}$ & $\begin{array}{l}\text { 3. } 342 \\
3.263 \\
3.207 \\
\text { 3. } 191 \\
\text { 3. } 083\end{array}$ & $\begin{array}{r}16 \\
18 \\
100 \\
100 \\
95\end{array}$ \\
\hline $\begin{array}{l}002 \\
131 \\
221 \\
040 \\
230\end{array}$ & $\begin{array}{l}3.037 \\
2.872 \\
2.838 \\
2.680 \\
2.664\end{array}$ & $\begin{array}{r}57 \\
4 \\
13 \\
12 \\
18\end{array}$ \\
\hline $\begin{array}{l}022 \\
310 \\
140 \\
122 \\
231\end{array}$ & $\begin{array}{l}2.644 \\
2.588 \\
2.541 \\
2.509 \\
2.442\end{array}$ & $\begin{array}{r}3 \\
30 \\
6 \\
11 \\
5\end{array}$ \\
\hline $\begin{array}{l}320 \\
311 \\
212 \\
141 \\
240\end{array}$ & $\begin{array}{l}\text { 2. } 388 \\
\text { 2. } 380 \\
\text { 2. } 359 \\
\text { 2. } 344 \\
\text { 2. } 226\end{array}$ & $\begin{array}{r}4 \\
14 \\
22 \\
14 \\
25\end{array}$ \\
\hline $\begin{array}{l}222 \\
330 \\
241 \\
150 \\
051\end{array}$ & $\begin{array}{l}\text { 2. } 205 \\
2.138 \\
2.091 \\
2.071 \\
2.021\end{array}$ & $\begin{array}{r}30 \\
11 \\
7 \\
12 \\
4\end{array}$ \\
\hline $\begin{array}{l}042 \\
232 \\
400 \\
312 \\
401\end{array}$ & $\begin{array}{l}\text { 2. } 0098 \\
\text { 2. } 0035 \\
\text { 2. } 0010 \\
\text { 1. } 9697 \\
\text { 1. } 8997\end{array}$ & $\begin{array}{l}11 \\
20 \\
18 \\
12 \\
10\end{array}$ \\
\hline $\begin{array}{c}340 \\
420 \\
411 \\
123 \\
341,251\end{array}$ & $\begin{array}{l}\text { 1. } 8908 \\
\text { 1. } 8737 \\
\text { 1. } 8703 \\
\text { 1. } 8426 \\
\text { 1. } 8047\end{array}$ & $\begin{array}{l}5 \\
7 \\
9 \\
6\end{array}$ \\
\hline $\begin{array}{l}060 \\
213 \\
033 \\
430\end{array}$ & $\begin{array}{l}\text { 1. } 7859 \\
\text { 1. } 7813 \\
\text { 1. } 7621 \\
\text { 1. } 7461\end{array}$ & $\begin{array}{r}6 \\
13 \\
15 \\
10\end{array}$ \\
\hline
\end{tabular}


Powder data cards. None.

Additional published patterns. Geller and Wernick [1] 1959.

NBS sample. The sample of silver antimony telluride was obtained from Semitronics Inc., Winchester, Mass. Spectrographic analysis showed the following major impurities: 0.01 to 0.1 percent each of aluminum, bismuth, copper, iron, indium, and tin; and 0.001 to 0.01 percent each of gold, calcium, chromium, magnesium, nickel, and titanium.

The sample was an opaque metallic gray powder.

The $d$-values of the three strongest lines are: $3.042,2.151$, and $1.7554 \AA$.

Structural data. Geller and Wernick [1] in 1959 determined that silver antimony telluride has the sodium chloride structure, the space group $\mathrm{O}_{\mathrm{h}}^{5}-\mathrm{Fm} 3 \mathrm{~m}$ (No. 225), and $2\left(\mathrm{AgSb}^{\prime} \mathrm{Te}_{2}\right)$ per unit cell.

\section{Lattice constants}

\begin{tabular}{|c|c|c|}
\hline & & $\AA$ \\
1959 & Geller and Wernick [1] at 25 ${ }^{\circ} \mathrm{C}-$ & 6.078 \\
1963 & $\begin{array}{r}\text { National Bureau of Standards at } \\
25{ }^{\circ} \mathrm{C} \ldots \ldots \ldots\end{array}$ & 6.080 \\
\hline
\end{tabular}

The density of silver antimony telluride calculated from the NBS lattice constant is 7.163 $\mathrm{g} / \mathrm{cm}^{3}$ at $25{ }^{\circ} \mathrm{C}$.

\begin{tabular}{|c|c|c|c|}
\hline \multirow[t]{2}{*}{$h k l$} & \multicolumn{3}{|c|}{$\begin{array}{l}\text { Internal Standard, } \\
\text { Tungsten, } a=3.1648 \AA \\
\mathrm{Cu}, 1.5405 \mathrm{~A} \text { at } 25^{\circ} \mathrm{C}\end{array}$} \\
\hline & $d$ & $I$ & $a$ \\
\hline $\begin{array}{l}111 \\
200 \\
220 \\
311 \\
222\end{array}$ & $\begin{array}{l}\quad \stackrel{\AA}{A} \\
\text { 3. } 511 \\
\text { 3. } 040 \\
\text { 2. } 151 \\
\text { 1. } 8332 \\
\text { 1. } 7554\end{array}$ & $\begin{array}{r}1 \\
100 \\
65 \\
<1 \\
19\end{array}$ & $\begin{array}{c}\stackrel{\AA}{A} \\
6.081 \\
6.080 \\
6.084 \\
6.080 \\
6.081\end{array}$ \\
\hline $\begin{array}{l}400 \\
331 \\
420 \\
422 \\
511\end{array}$ & $\begin{array}{l}\text { 1. } 5203 \\
\text { 1. } 3946 \\
\text { 1. } 3597 \\
\text { 1. } 2411 \\
\text { 1. } 1703\end{array}$ & $\begin{array}{r}9 \\
<1 \\
18 \\
9 \\
<1\end{array}$ & $\begin{array}{l}\text { 6. } 081 \\
6.079 \\
6.081 \\
6.079 \\
6.081\end{array}$ \\
\hline $\begin{array}{l}440 \\
531 \\
600 \\
620 \\
622\end{array}$ & $\begin{array}{r}1.0748 \\
1.0276 \\
1.0133 \\
0.9614 \\
.9166\end{array}$ & $\begin{array}{r}4 \\
<1 \\
4 \\
3 \\
2\end{array}$ & $\begin{array}{l}6.080 \\
6.079 \\
6.080 \\
6.080 \\
6.080\end{array}$ \\
\hline $\begin{array}{l}444 \\
711 \\
640 \\
642\end{array}$ & $\begin{array}{l}.8775 \\
.8513 \\
.8433 \\
.8125\end{array}$ & $\begin{array}{r}1 \\
1 \\
2 \\
2\end{array}$ & $\begin{array}{l}\text { 6. } 079 \\
\text { 6. } 079 \\
\text { 6. } 081 \\
\text { 6. } 080\end{array}$ \\
\hline
\end{tabular}

Average value of last five lines

6. 080

\section{Reference}

[1] S. Geller and J. H. Wernick, Ternary semiconducting compounds with sodium chloride-like structure: $\mathrm{AgSbSe}_{2}, \mathrm{AgSbTe}_{2}, \mathrm{AgBiS}_{2}, \mathrm{AgBiSe}_{2}$, Acta Cryst. 12, 46-54 (1959).

\section{Sodium Magnesium Aluminum Boron Hydroxy Silicate, dravite (var. of tourmaline), $\mathrm{NaMg}_{3} \mathrm{Al}_{6} \mathrm{~B}_{3} \mathrm{Si}_{6} \mathrm{O}_{27}(\mathrm{OH})_{4}$ (trigonal)}

Powder Data cards. None. Additional published patterns. None.

NBS sample. The sample of dravite is U.S. National Museum No. 103791 from Dobruva, Carinthia, Austria. This mineral sample was picked by C. R. Robbins, NBS, as a true end member representative of the magnesium rich variety of tourmaline. The chemical analysis by H. B. Wiik, Hilsingfors, Westend, Finland, showed the following: 36.99 percent $\mathrm{SiO}_{2} ; 0.39$ percent $\mathrm{TiO}_{2} ; 32.00$ percent $\mathrm{Al}_{2} \mathrm{O}_{3} ; 0.01$ percent $\mathrm{MnO} ; 11.58$ percent $\mathrm{MgO} ; 0.50$ percent $\mathrm{CaO}$; 0.01 percent $\mathrm{Li}_{2} \mathrm{O} ; 3.11$ percent $\mathrm{Na}_{2} \mathrm{O} ; 0.08$ percent $\mathrm{K}_{2} \mathrm{O} ; 3.08$ percent $\mathrm{H}_{2} \mathrm{O}^{+} ; 10.77$ percent $\mathrm{B}_{2} \mathrm{O}_{3}$; 0.25 percent $\mathrm{F}$; and 0.90 percent total $\mathrm{Fe}$ as $\mathrm{FeO}$.

The color of the sample was light brown and it is optically negative. The indices of refraction are $\mathrm{N}_{0}=1.634$ and $\mathrm{N}_{\mathrm{e}}=1.613$.

The $d$-values of the three strongest lines are: $2.576,3.985$, and $2.961 \AA$.
Structural data. Buerger and Parrish [1] in 1937 determined the structure of the tourmalines; they have the space group $\mathrm{C}_{3 \mathrm{v}}^{5}-\mathrm{R} 3 \mathrm{~m}$ (No. 160) and $3\left[\mathrm{NaMg}_{3} \mathrm{Al}_{6} \mathrm{~B}_{3} \mathrm{Si}_{6} \mathrm{O}_{27}(\mathrm{OH})_{4}\right]$ per unit hexagonal cell.

\section{Lattice constants}

\begin{tabular}{|c|c|c|c|}
\hline \multirow{5}{*}{$\begin{array}{l}1948 \\
1949 \\
1951 \\
1963\end{array}$} & \multirow{2}{*}{$\begin{array}{l}\text { Hamburger and Buerger } \\
{[2]}\end{array}$} & $a$ & $c$ \\
\hline & & $\stackrel{\AA}{A}$ & $\AA$ \\
\hline & Belova and Belova [3] & 16. 00 & 7. 24 \\
\hline & Kurylenko [4] & 15. 676 & 7. 03 \\
\hline & $\begin{array}{l}\text { National Bureau of } \\
\text { Standards at } 25{ }^{\circ} \mathrm{C}_{-}\end{array}$ & 15. 931 & 7. 197 \\
\hline
\end{tabular}

The density of dravite calculated from NBS lattice constants is $3.019 \mathrm{~g} / \mathrm{cm}^{3}$ at $25^{\circ} \mathrm{C}$. 
Sodium Magnesium Aluminum Boron Hydroxy Silicate, dravite (var. of tourmaline), $\mathrm{NaMg}_{3} \mathrm{Al}_{6} \mathrm{~B}_{3} \mathrm{Si}_{6} \mathrm{O}_{27}(\mathrm{OH})_{4}$ (trigonal)-Continued

\begin{tabular}{|c|c|c|}
\hline \multirow{2}{*}{$\begin{array}{l}h k l \\
\text { (hex) }\end{array}$} & \multicolumn{2}{|c|}{$\begin{array}{l}\text { Internal Standard } \\
\text { Tungsten, } a=3.1648 \AA \\
\mathrm{Cu}, 1.5405 \AA \text { at } 25^{\circ} \mathrm{C}\end{array}$} \\
\hline & $d$ & $I$ \\
\hline $\begin{array}{l}101 \\
021 \\
300 \\
211 \\
220\end{array}$ & $\begin{array}{c}\stackrel{\AA}{A} \\
6.377 \\
4.981 \\
4.595 \\
4.221 \\
\text { 3. } 985\end{array}$ & $\begin{array}{l}28 \\
25 \\
17 \\
66 \\
84\end{array}$ \\
\hline $\begin{array}{l}012 \\
131 \\
401 \\
410 \\
122\end{array}$ & $\begin{array}{l}\text { 3. } 480 \\
\text { 3. } 375 \\
\text { 3. } 111 \\
\text { 3. } 008 \\
\text { 2. } 961\end{array}$ & $\begin{array}{r}62 \\
16 \\
5 \\
12 \\
83\end{array}$ \\
\hline $\begin{array}{l}321 \\
330 \\
312 \\
051 \\
042\end{array}$ & $\begin{array}{l}\text { 2. } 897 \\
\text { 2. } 656 \\
\text { 2. } 622 \\
\text { 2. } 576 \\
\text { 2. } 490\end{array}$ & $\begin{array}{r}9 \\
<1 \\
7 \\
100 \\
1\end{array}$ \\
\hline $\begin{array}{l}241 \\
003 \\
232 \\
511 \\
600\end{array}$ & $\begin{array}{l}\text { 2. } 451 \\
\text { 2. } 396 \\
\text { 2. } 376 \\
\text { 2. } 342 \\
\text { 2. } 300\end{array}$ & $\begin{array}{r}1 \\
20 \\
19 \\
21 \\
5\end{array}$ \\
\hline $\begin{array}{l}502 \\
431 \\
303 \\
422 \\
223\end{array}$ & $\begin{array}{l}\text { 2. } 189 \\
\text { 2. } 163 \\
\text { 2. } 127 \\
\text { 2. } 112 \\
\text { 2. } 054\end{array}$ & $\begin{array}{l}17 \\
14 \\
16 \\
10 \\
21\end{array}$ \\
\hline $\begin{array}{l}152 \\
161 \\
440 \\
342 \\
701\end{array}$ & $\begin{array}{l}\text { 2. } 040 \\
\text { 2. } 019 \\
\text { 1. } 991 \\
\text { 1. } 920 \\
\text { 1. } 901\end{array}$ & $\begin{array}{r}46 \\
7 \\
5 \\
34 \\
6\end{array}$ \\
\hline $\begin{array}{l}413 \\
621 \\
710 \\
612 \\
104\end{array}$ & $\begin{array}{l}\text { 1. } 877 \\
\text { 1. } 849 \\
\text { 1. } 828 \\
\text { 1. } 817 \\
\text { 1. } 784\end{array}$ & $\begin{array}{l}7 \\
8 \\
2 \\
2 \\
9\end{array}$ \\
\hline
\end{tabular}

\begin{tabular}{|c|c|c|}
\hline \multirow{2}{*}{$\begin{array}{c}h k l \\
(h e x)\end{array}$} & \multicolumn{2}{|c|}{$\begin{array}{l}\text { Internal Standard } \\
\text { Tungsten, } a=3.1648 \AA \\
\mathrm{Cu}, 1.5405 \AA \text { at } 25^{\circ} \mathrm{C}\end{array}$} \\
\hline & $d$ & $I$ \\
\hline $\begin{array}{l}333 \\
024 \\
532 \\
541 \\
262\end{array}$ & $\begin{array}{c}\stackrel{\circ}{A} \\
1.781 \\
1.742 \\
1.729 \\
1.715 \\
1.690\end{array}$ & $\begin{array}{l}7 \\
5 \\
4 \\
2 \\
1\end{array}$ \\
\hline $\begin{array}{l}603 \\
271 \\
550 \\
452 \\
811\end{array}$ & $\begin{array}{l}1.660 \\
1.641 \\
1.592 \\
1.586 \\
1.575\end{array}$ & $\begin{array}{r}24 \\
16 \\
21 \\
<1 \\
<1\end{array}$ \\
\hline $\begin{array}{l}324 \\
461 \\
900 \\
722 \\
820\end{array}$ & $\begin{array}{l}1.565 \\
1.5456 \\
1.5326 \\
1.5262 \\
1.5056\end{array}$ & $\begin{array}{r}<1 \\
5 \\
7 \\
7 \\
16\end{array}$ \\
\hline $\begin{array}{l}244 \\
514 \\
642 \\
015 \\
651\end{array}$ & $\begin{array}{l}\text { 1. } 4807 \\
\text { 1. } 4555 \\
\text { 1. } 4485 \\
\text { 1. } 4318 \\
\text { 1. } 4178\end{array}$ & $\begin{array}{r}3 \\
19 \\
11 \\
8 \\
8\end{array}$ \\
\hline $\begin{array}{c}205 \\
125 \\
381 \\
10 \cdot 0 \cdot 1 \\
912\end{array}$ & $\begin{array}{l}\text { 1. } 4091 \\
\text { 1. } 3871 \\
\text { 1. } 3746 \\
\text { 1. } 3551 \\
\text { 1. } 3416\end{array}$ & $\begin{array}{r}19 \\
2 \\
3 \\
9 \\
5\end{array}$ \\
\hline $\begin{array}{c}921 \\
045 \\
660,553 \\
10.1 .0 \\
571\end{array}$ & $\begin{array}{l}\text { 1. } 3359 \\
\text { 1. } 3282 \\
\text { 1. } 3272 \\
\text { 1. } 3095 \\
\text { 1. } 3002\end{array}$ & $\begin{array}{r}4 \\
13 \\
12 \\
12 \\
1\end{array}$ \\
\hline $\begin{array}{c}903 \\
505 \\
425 \\
155 \\
0.11 .1\end{array}$ & $\begin{array}{l}\text { 1. } 2922 \\
\text { 1. } 2765 \\
\text { 1. } 2602 \\
\text { 1. } 2449 \\
1.2358\end{array}$ & $\begin{array}{r}2 \\
14 \\
3 \\
3 \\
3\end{array}$ \\
\hline $\begin{array}{l}482 \\
850\end{array}$ & $\begin{array}{l}\text { 1. } 2260 \\
\text { 1. } 2149\end{array}$ & $\begin{array}{l}1 \\
3\end{array}$ \\
\hline
\end{tabular}

\section{References}

[1] M. J. Buerger and W. Parrish, The unit cell and space group of tourmaline (an example of the inspective equiinclination treatment of trigonal crystals), Am. Mineralogist 22, 1139-1150 (1937).

[2] G. E. Hamburger and M. J. Buerger, The structure of tourmaline, Am. Mineralogist 33, 532-540 (1948).
[3] N. V. Belova and E. N. Belova (The crystal structure of tourmaline), Dokl. Akad. Nauk SSSR 69, No. 2, 185-188 (1949).

[4] C. Kurylenko, Transformation de la dravite de Doubrova (Moravie) de $375^{\circ}$ à $1350^{\circ}$, Compt. rend. Paris 232, 2109-2111 (1951). 
Powder data cards.

\begin{tabular}{|c|c|c|}
\hline \hline $\begin{array}{c}\text { Card } \\
\text { number }\end{array}$ & $\begin{array}{c}\text { Index } \\
\text { lines }\end{array}$ & Source \\
\cline { 2 - 3 } 11-648 & $\begin{array}{r}3.40 \\
3.86 \\
3.04\end{array}$ & Dienst, Delft, Holland. \\
& de Wolf, Techn. Phys. \\
\hline
\end{tabular}

Additional published patterns. Corbridge and Tromans [1].

NBS sample. The sample of sodium trimetaphosphate was crystallized by heating sodium trimetaphosphate above the melting point (about $650^{\circ} \mathrm{C}$ ) and then cooling slowly at the rate of 5 to $10{ }^{\circ} \mathrm{C}$ an hour. Spectrographic analysis showed the following major impurities: 0.001 to 0.01 percent each of aluminum, barium, calcium, iron, potassium, magnesium, silicon, and titanium.

The sample is colorless.

\begin{tabular}{|c|c|c|}
\hline \multirow[t]{3}{*}{$h k l$} & \multicolumn{2}{|c|}{$\begin{array}{l}\text { Internal Standard, } \\
\text { Tungsten, } a=3.1648 \AA \\
\mathrm{Cu}, 1.5405 \AA \text { at } 25^{\circ} \mathrm{C}\end{array}$} \\
\hline & $d$ & $I$ \\
\hline & $\AA$ & \\
\hline $\begin{array}{l}110 \\
011 \\
111 \\
021 \\
121\end{array}$ & $\begin{array}{l}6.79 \\
6.65 \\
5.095 \\
5.015 \\
4.237\end{array}$ & $\begin{array}{r}43 \\
53 \\
43 \\
48 \\
5\end{array}$ \\
\hline $\begin{array}{c}200 \\
002,130 \\
131 \\
211 \\
112\end{array}$ & $\begin{array}{l}\text { 3. } 960 \\
3.850 \\
3.444 \\
\text { 3. } 407 \\
\text { 3. } 351\end{array}$ & $\begin{array}{r}16 \\
63 \\
48 \\
100 \\
31\end{array}$ \\
\hline $\begin{array}{l}022 \\
221 \\
041 \\
141 \\
202\end{array}$ & $\begin{array}{l}\text { 3. } 330 \\
\text { 3. } 110 \\
\text { 3. } 037 \\
\text { 2. } 837 \\
\text { 2. } 762\end{array}$ & $\begin{array}{l}37 \\
11 \\
55 \\
11 \\
41\end{array}$ \\
\hline $\begin{array}{l}132 \\
212 \\
310 \\
240 \\
013\end{array}$ & $\begin{array}{l}2.723 \\
2.706 \\
2.591 \\
2.540 \\
2.522\end{array}$ & $\begin{array}{l}22 \\
10 \\
11 \\
35 \\
14\end{array}$ \\
\hline $\begin{array}{c}150,051 \\
311 \\
241 \\
113 \\
151\end{array}$ & $\begin{array}{l}2.503 \\
2.457 \\
2.412 \\
2.406 \\
\text { 2. } 387\end{array}$ & $\begin{array}{r}7 \\
8 \\
11 \\
8 \\
<1\end{array}$ \\
\hline $\begin{array}{c}232,321 \\
123 \\
330 \\
060 \\
331\end{array}$ & $\begin{array}{l}\text { 2. } 340 \\
\text { 2. } 292 \\
\text { 2. } 267 \\
\text { 2. } 204 \\
\text { 2. } 175\end{array}$ & $\begin{array}{r}<1 \\
2 \\
6 \\
8 \\
11\end{array}$ \\
\hline
\end{tabular}

The $d$-values of the three strongest lines are: $3.407,3.850$, and $3.037 \AA$.

Structural data. Ondik and Gryder [2] in 1960 determined that sodium trimetaphosphate has the space group $\mathrm{C}_{2 \mathrm{v}}^{9}-\mathrm{P} 2_{1} \mathrm{cn}$ (No. 33) or $\mathrm{D}_{2 \mathrm{~h}}^{16}-\mathrm{Pmen}$ $(\mathrm{No} .62)$ with $4\left(\mathrm{Na}_{3} \mathrm{P}_{3} \mathrm{O}_{9}\right)$ per unit cell.

\begin{tabular}{|c|c|c|}
\hline \multirow[t]{2}{*}{$h k l$} & \multicolumn{2}{|c|}{$\begin{array}{l}\text { Internal Standard, } \\
\text { Tungsten } a=3.1648 \AA \\
\mathrm{Cu}, 1.5405 \AA \text { at } 25^{\circ} \mathrm{C}\end{array}$} \\
\hline & $d$ & $I$ \\
\hline $\begin{array}{l}133 \\
213 \\
242 \\
152 \\
223\end{array}$ & $\begin{array}{ll} & \AA \\
2 . & 136 \\
2 . & 128 \\
2 . & 121 \\
2 . & 103 \\
2 . & 050\end{array}$ & $\begin{array}{r}8 \\
10 \\
8 \\
2 \\
10\end{array}$ \\
\hline $\begin{array}{l}043 \\
341 \\
400 \\
143 \\
332\end{array}$ & $\begin{array}{l}2.029 \\
1.994 \\
1.982 \\
1.965 \\
1.954\end{array}$ & $\begin{array}{r}17 \\
6 \\
9 \\
5 \\
6\end{array}$ \\
\hline $\begin{array}{c}004,260 \\
252 \\
411,420 \\
350 \\
024\end{array}$ & $\begin{array}{l}1.927 \\
1.911 \\
1.900 \\
1.871 \\
1.849\end{array}$ & $\begin{array}{r}5 \\
8 \\
1 \\
13 \\
10\end{array}$ \\
\hline $\begin{array}{l}170 \\
243 \\
153 \\
171 \\
402\end{array}$ & $\begin{array}{l}1.838 \\
1.806 \\
1.794 \\
1.781 \\
1.762\end{array}$ & $\begin{array}{l}4 \\
4 \\
2 \\
1 \\
1\end{array}$ \\
\hline $\begin{array}{l}134 \\
214 \\
333 \\
352 \\
224\end{array}$ & $\begin{array}{l}1.723 \\
\text { 1. } 719 \\
1.699 \\
\text { 1. } 681 \\
1.675\end{array}$ & $\begin{array}{r}1 \\
<1 \\
2 \\
5 \\
8\end{array}$ \\
\hline $\begin{array}{c}063,253 \\
271,044 \\
441 \\
144 \\
234\end{array}$ & $\begin{array}{l}\text { 1. } 671 \\
1.665 \\
1.661 \\
1.629 \\
1.612\end{array}$ & $\begin{array}{l}8 \\
5 \\
5 \\
1 \\
6\end{array}$ \\
\hline $\begin{array}{l}181 \\
054 \\
451 \\
073 \\
082\end{array}$ & $\begin{array}{l}1.583 \\
1.557 \\
1.554 \\
1.521 \\
1.518\end{array}$ & $\begin{array}{l}2 \\
5 \\
7 \\
3 \\
2\end{array}$ \\
\hline $\begin{array}{l}353 \\
371 \\
025 \\
531 \\
254\end{array}$ & $\begin{array}{l}\text { 1. } 511 \\
\text { 1. } 507 \\
\text { 1. } 501 \\
\text { 1. } 465 \\
1.449\end{array}$ & $\begin{array}{l}5 \\
6 \\
6 \\
3 \\
5\end{array}$ \\
\hline $\begin{array}{l}372 \\
282\end{array}$ & $\begin{array}{l}\text { 1. } 427 \\
\text { 1. } 418\end{array}$ & $\begin{array}{r}11 \\
2\end{array}$ \\
\hline
\end{tabular}




\section{Sodium Trimetaphosphate, $\mathrm{Na}_{3} \mathrm{P}_{3} \mathrm{O}_{9}$ (orthorhombic)-Continued}

Lattice constants

\begin{tabular}{|c|c|c|c|c|}
\hline & & $a$ & $b$ & $c$ \\
\hline & & $\AA$ & $\AA$ & $\AA$ \\
\hline 1960 & $\begin{array}{l}\text { Ondik and Gry- } \\
\text { der [2]. }\end{array}$ & 7.93 & 13. 14 & 7. 75 \\
\hline 1962 & de Wolff & 7.928 & 13. 22 & 7. 703 \\
\hline 1963 & $\begin{array}{l}\text { National Bureau } \\
\text { of Standards } \\
\text { at } 25^{\circ} \mathrm{C} \text {. }\end{array}$ & 7. 930 & 13. 220 & 7. 705 \\
\hline
\end{tabular}

The density of sodium trimetaphosphate calculated from the NBS lattice constants is 2.515 $\mathrm{g} / \mathrm{cm}^{3}$ at $25{ }^{\circ} \mathrm{C}$.

\section{References}

[1] D. E. C. Corbridge and F. R. Tromans, Identification of sodium phosphates with an x-ray focusing camera, Anal. Chem. 30, 1101-1110 (1958)

[2] H. M. Ondik and J. W. Gryder, Crystal chemistry of the hydrates of sodium trimetaphosphate, J. Inorg. Nucl. Chem. 14, Nos. 3/4, 240-246 (1960).

\section{Sodium Trimetaphosphate Monohydrate, $\mathrm{Na}_{3} \mathrm{P}_{3} \mathrm{O}_{9} \cdot \mathrm{H}_{2} \mathrm{O}$ (orthohombic)}

\section{Powder data cards}

\begin{tabular}{|c|c|c|}
\hline $\begin{array}{c}\text { Card } \\
\text { number }\end{array}$ & $\begin{array}{c}\text { Index } \\
\text { lines }\end{array}$ & Source \\
\hline $1-0977$ & $\begin{array}{l}2.84 \\
3.03 \\
2.60\end{array}$ & New Jersey Zinc Co. \\
& $\begin{array}{l}4.96 \\
11-391\end{array}$ & Corbridge and Tromans [1] 1958. \\
& $\begin{array}{l}3.57 \\
3.01\end{array}$ & \\
\hline
\end{tabular}

Card number 12-10 also is called $\mathrm{Na}_{3} \mathrm{P}_{3} \mathrm{O}_{9} \cdot \mathrm{H}_{2} \mathrm{O}$; however, it contains a very different pattern, perhaps another form.

Additional published patterns. None.

NBS sample. The sample of sodium trimetaphosphate monohydrate was prepared at NBS by heating sodium dihydrogen orthophosphate for $5 \mathrm{hr}$ at $530{ }^{\circ} \mathrm{C}$. Solid sodium trimetaphosphate was salted out with $\mathrm{NaCl}$ and then recrystallized several times. Spectrographic analysis showed the following major impurities: 0.001 to 0.01 percent each of aluminum, barium, calcium, iron, potassium, magnesium, silicon, and titanium.

The sample is colorless and it is probably optically negative. The indices of refraction are $\mathrm{N}_{\alpha}=1.493, \mathrm{~N}_{\beta}=1.502$, and $\mathrm{N}_{\gamma}=1.504$.

The $d$-values of the three strongest lines are: $3.566,2.825$, and $(2.605$ and 2.601$) \AA$

Structural data. Ondik and Gryder [2] in 1960 determined that sodium trimetaphosphate monohydrate has the space group $\mathrm{C}_{2 \mathrm{v}}^{9}-\mathrm{P2}_{1} \mathrm{cn}$ (No. 33) or $\mathrm{D}_{2 \mathrm{~h}}^{16} \mathrm{Pmcn}(\mathrm{No} .62)$ with $4\left(\mathrm{Na}_{3} \mathrm{P}_{3} \mathrm{O}_{9} \cdot \mathrm{H}_{2} \mathrm{O}\right)$ per unit cell.

\begin{tabular}{|c|c|c|}
\hline \multirow[t]{2}{*}{$h k l$} & \multicolumn{2}{|c|}{$\begin{array}{l}\text { Internal Standard, } \\
\text { Tungsten, } a=3.1648 \AA \\
\mathrm{Cu}, 1.5405 \AA \text { at } 25^{\circ} \mathrm{C}\end{array}$} \\
\hline & $d$ & $I$ \\
\hline $\begin{array}{l}110 \\
011 \\
111 \\
021 \\
121\end{array}$ & 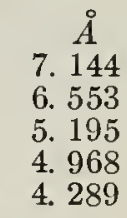 & $\begin{array}{l}42 \\
38 \\
49 \\
60 \\
32\end{array}$ \\
\hline $\begin{array}{l}200 \\
130 \\
002 \\
012 \\
211\end{array}$ & $\begin{array}{l}\text { 4. } 252 \\
\text { 3. } 905 \\
\text { 3. } 778 \\
\text { 3. } 633 \\
\text { 3. } 566\end{array}$ & $\begin{array}{r}5 \\
32 \\
17 \\
15 \\
100\end{array}$ \\
\hline $\begin{array}{l}131 \\
112 \\
022 \\
221 \\
122\end{array}$ & $\begin{array}{l}\text { 3. } 469 \\
\text { 3. } 340 \\
\text { 3. } 278 \\
\text { 3. } 230 \\
\text { 3. } 060\end{array}$ & $\begin{array}{r}64 \\
60 \\
55 \\
8 \\
8\end{array}$ \\
\hline $\begin{array}{l}041 \\
141 \\
202 \\
310 \\
212\end{array}$ & $\begin{array}{l}\text { 3. } 022 \\
\text { 2. } 848 \\
\text { 2. } 825 \\
\text { 2. } 769 \\
\text { 2. } 762\end{array}$ & $\begin{array}{r}73 \\
5 \\
100 \\
57\end{array}$ \\
\hline $\begin{array}{l}132 \\
240 \\
311 \\
150 \\
051\end{array}$ & $\begin{array}{l}\text { 2. } 716 \\
2.605 \\
2.601 \\
2.520 \\
2.491\end{array}$ & $\begin{array}{r}10 \\
75 \\
5 \\
18\end{array}$ \\
\hline $\begin{array}{c}042 \\
013 \\
330 \\
113,232 \\
331\end{array}$ & $\begin{array}{l}\text { 2. } 484 \\
\text { 2. } 474 \\
\text { 2. } 382 \\
\text { 2. } 375 \\
\text { 2. } 272\end{array}$ & $\begin{array}{r}21 \\
39 \\
6 \\
17\end{array}$ \\
\hline $\begin{array}{c}123,302 \\
312 \\
060 \\
052 \\
213\end{array}$ & $\begin{array}{l}\text { 2. } 266 \\
\text { 2. } 235 \\
\text { 2. } 198 \\
\text { 2. } 163 \\
\text { 2. } 139\end{array}$ & $\begin{array}{r}4 \\
4 \\
16 \\
<2 \\
15\end{array}$ \\
\hline
\end{tabular}




\section{Sodium Trimetaphosphate Monohydrate, $\mathrm{Na}_{3} \mathrm{P}_{3} \mathrm{O}_{9} \cdot \mathrm{H}_{2} \mathrm{O}$ (orthorhombic)-Continued}

\begin{tabular}{|c|c|c|}
\hline \multirow[t]{2}{*}{$h k l$} & \multicolumn{2}{|c|}{$\begin{array}{c}\text { Internal Standard, } \\
\text { Tungsten, } a=3.1648 \AA \\
\mathrm{Cu}, 1.5405 \AA \text { at } 25^{\circ} \mathrm{C}\end{array}$} \\
\hline & $d$ & $I$ \\
\hline $\begin{array}{l}400 \\
133 \\
341 \\
420 \\
332\end{array}$ & $\begin{array}{l}\stackrel{\AA}{A} \\
\text { 2. } 125 \\
\text { 2. } 116 \\
\text { 2. } 067 \\
\text { 2. } 022 \\
\text { 2. } 016\end{array}$ & $\begin{array}{r}17 \\
12 \\
8 \\
5 \\
10\end{array}$ \\
\hline $\begin{array}{c}043 \\
421,260 \\
143 \\
350 \\
252\end{array}$ & $\begin{array}{l}\text { 2. } 001 \\
\text { 1. } 954 \\
\text { 1. } 949 \\
\text { 1. } 930 \\
1.927\end{array}$ & $\begin{array}{r}12 \\
29 \\
12 \\
13 \\
9\end{array}$ \\
\hline $\begin{array}{c}062 \\
261,004 \\
351 \\
162,431 \\
170,412\end{array}$ & $\begin{array}{l}\text { 1. } 900 \\
\text { 1. } 889 \\
\text { 1. } 870 \\
\text { 1. } 855 \\
\text { 1. } 840\end{array}$ & $\begin{array}{r}6 \\
11 \\
9 \\
4 \\
6\end{array}$ \\
\hline $\begin{array}{c}053 \\
024 \\
243,323 \\
171,440 \\
153\end{array}$ & $\begin{array}{l}\text { 1. } 823 \\
\text { 1. } 817 \\
\text { 1. } 811 \\
\text { 1. } 786 \\
\text { 1. } 781\end{array}$ & $\begin{array}{r}9 \\
7 \\
3 \\
11 \\
10\end{array}$ \\
\hline $\begin{array}{l}352 \\
253 \\
413\end{array}$ & $\begin{array}{l}\text { 1. } 719 \\
\text { 1. } 674 \\
\text { 1. } 612\end{array}$ & $\begin{array}{l}16 \\
17 \\
10\end{array}$ \\
\hline
\end{tabular}

Lattice constants

\begin{tabular}{|c|c|c|c|c|}
\hline & & $a$ & $b$ & $c$ \\
\cline { 2 - 4 } 1960 & $\AA$ & $\AA$ & $\AA$ \\
1963 & $\begin{array}{c}\text { Ondik and } \\
\text { Gryder [2]. } \\
\text { National Bureau } \\
\text { of Standards } \\
\text { at 25 }{ }^{\circ} \mathrm{C} .\end{array}$ & 8.53 & 13.21 & 7.58 \\
\hline 0.001 & 8.500 & 13.189 & 7.558 \\
\pm 0.001 & \pm 0.001 \\
\hline
\end{tabular}

The density of sodium trimetaphosphate monohydrate calculated from NBS lattice constants is $2.539 \mathrm{~g} / \mathrm{cm}^{3}$ at $25{ }^{\circ} \mathrm{C}$.

\section{References}

[1] D. E. C. Corbridge and F. R. Tromans, Identification of sodium phosphates with an x-ray focusing camera, Anal. Chem. 30, No. 6, 1101-1110 (1958).

[2] H. Ondik and J. W. Gryder, Crystal chemistry of the hydrates of sodium trimetaphosphate, J. Inorg. Nucl. Chem. 14, 240-246 (1960)

\section{Stannous Fluoride, $\mathrm{SnF}_{2}$ (monoclinic)}

Powder data cards. None

Additional published patterns. Nebergall, Muhler, and Day [1] 1952.

NBS sample. The sample of stannous fluoride was obtained from the Indiana University School of Dentistry, Indianapolis, Ind. Spectrographic analysis showed the following major impurities: 0.001 to 0.01 percent each of calcium and silicon.

The sample was colorless. The indices of refraction were not determined because the sample reacted with the index liquids.
The $d$-values of the three strongest lines are: $3.552,3.200$ and $3.379 \AA$.

Structural data. Bergerhoff [2] in 1962 determined that stannous fluoride has the space group $\mathrm{C}_{2 \mathrm{~h}}^{6}-\mathrm{C} 2 / \mathrm{c}(\mathrm{No} .15)$ and $16\left[\mathrm{SnF}_{2}\right]$ per unit cell.

Difficulties in indexing the NBS patter'n were overcome with the help of single crystal work done by Dr. Howard T. Evans Jr., of the U.S. Geological Survey.

Lattice constants

\begin{tabular}{|c|c|c|c|c|c|}
\hline & & $a$ & $b$ & $c$ & $\beta$ \\
\hline $\begin{array}{l}1962 \\
1963\end{array}$ & $\begin{array}{l}\text { Bergerhoff }[2] \\
\text { National Bureau of Standards } \\
\quad \text { at } 25^{\circ} \mathrm{C}\end{array}$ & $\begin{array}{c}\stackrel{\AA}{A} \\
13.46 \\
13.353 \\
\pm .001\end{array}$ & $\begin{array}{c}\stackrel{\circ}{A} \\
4.92 \\
4.9089 \\
\pm .0004\end{array}$ & $\begin{array}{c}\stackrel{\AA}{A} \\
13.86 \\
13.787 \\
\pm .001\end{array}$ & $\begin{array}{l}109^{\circ} 30^{\prime} \\
019^{\circ} 6.5^{\prime} \\
\pm .3^{\prime}\end{array}$ \\
\hline
\end{tabular}




\section{Stannous Fluoride, $\mathrm{SnF}_{2}$ (monoclinic) - Continued}

\begin{tabular}{|c|c|c|}
\hline \multirow[t]{2}{*}{$h k l$} & \multicolumn{2}{|c|}{$\begin{array}{c}\text { Internal Standard, } \\
\text { Tungsten, } a=3.1648 \AA \\
\mathrm{Cu}, 1.5405 \AA \text { at } 25{ }^{\circ} \mathrm{C}\end{array}$} \\
\hline & $d$ & $I$ \\
\hline $\begin{array}{l}\overline{1} 11 \\
112 \\
\frac{2}{2} 04 \\
\frac{3}{3} 11\end{array}$ & $\begin{array}{c}\quad \stackrel{\circ}{A} \\
\text { 4. } 489 \\
3.552 \\
3.379 \\
3.298 \\
\text { 3. } 200\end{array}$ & $\begin{array}{r}5 \\
100 \\
40 \\
2 \\
60\end{array}$ \\
\hline $\begin{array}{l}400 \\
204 \\
020 \\
021 \\
511\end{array}$ & $\begin{array}{l}\text { 3. } 155 \\
\text { 2. } 571 \\
\text { 2. } 455 \\
\text { 2. } 412 \\
\text { 2. } 327\end{array}$ & $\begin{array}{r}34 \\
11 \\
9 \\
1 \\
2\end{array}$ \\
\hline $\begin{array}{l}\overline{3} 15 \\
313 \\
\overline{6} 02 \\
\overline{6} 04 \\
222\end{array}$ & $\begin{array}{l}\text { 2. } 320 \\
\text { 2. } 315 \\
\text { 2. } 225 \\
\text { 2. } 109 \\
\text { 2. } 084\end{array}$ & $\begin{array}{r}3 \\
2 \\
1 \\
10 \\
<1\end{array}$ \\
\hline $\begin{array}{l}\overline{1} 16 \\
\frac{3}{3} 16 \\
512 \\
420\end{array}$ & $\begin{array}{l}\text { 2. } 059 \\
\text { 2. } 048 \\
\text { 1. } 986 \\
\text { 1. } 954 \\
\text { 1. } 938\end{array}$ & $\begin{array}{r}18 \\
2 \\
16 \\
16 \\
18\end{array}$ \\
\hline $\begin{array}{c}\overline{5} 16 \\
602 \\
\overline{7} 1 \frac{2}{4}, 224 \\
408 \\
008\end{array}$ & $\begin{array}{l}1.854 \\
1.833 \\
1.776 \\
1.690 \\
1.629\end{array}$ & $\begin{array}{r}12 \\
<1 \\
22 \\
2 \\
5\end{array}$ \\
\hline $\begin{array}{c}\overline{1} 31,316 \\
624 \\
800 \\
132 \\
604\end{array}$ & $\begin{array}{l}\text { 1. } 6186 \\
\text { 1. } 5997 \\
1.5769 \\
1.5594 \\
1.5504\end{array}$ & $\begin{array}{l}6 \\
6 \\
4 \\
5 \\
3\end{array}$ \\
\hline
\end{tabular}

The density of stannous fluoride calculated from the NBS lattice constants is $4.875 \mathrm{~g} / \mathrm{cm}^{3}$ at $25^{\circ} \mathrm{C}$.

\begin{tabular}{|c|c|c|}
\hline \multirow[t]{2}{*}{$h k l$} & \multicolumn{2}{|c|}{$\begin{array}{c}\text { Internal Standard, } \\
\text { Tungsten, } a=3.1648 \AA \\
\mathrm{Cu}, 1.5405 \AA \text { at } 25^{\circ} \mathrm{C}\end{array}$} \\
\hline & $d$ & $I$ \\
\hline $\begin{array}{l}332 \\
912 \\
428 \\
808 \\
028\end{array}$ & $\begin{array}{c}\AA \\
\text { 1. } 5258 \\
1.4112 \\
1.3921 \\
1.3812 \\
1.3570\end{array}$ & $\begin{array}{r}5 \\
<1 \\
4 \\
1 \\
2\end{array}$ \\
\hline $\begin{array}{c}\overline{9} 16 \\
\overline{10} \cdot 0 \cdot 4 \\
\overline{3} \cdot 1 \cdot 10, \overline{1} 36,820 \\
\overline{5} \cdot 1 \cdot 10, \frac{624}{826,0 \cdot 0 \cdot 10}\end{array}$ & $\begin{array}{l}\text { 1. } 3517 \\
\text { 1. } 3325 \\
\text { 1. } 3271 \\
\text { 1. } 3107 \\
\text { 1. } 3031\end{array}$ & $\begin{array}{r}2 \\
1 \\
5 \\
2 \\
<1\end{array}$ \\
\hline $\begin{array}{l}532 \\
408 \\
536 \\
912 \\
732\end{array}$ & $\begin{array}{l}\text { 1. } 2974 \\
\text { 1. } 2858 \\
\text { 1. } 2667 \\
\text { 1. } 2446 \\
\text { 1. } 2411\end{array}$ & $\begin{array}{l}2 \\
1 \\
2 \\
1 \\
2\end{array}$ \\
\hline $\begin{array}{c}\overline{7} \cdot 1 \cdot 10, \overline{7} 31 \\
040, \overline{734} \\
1 \cdot 1 \cdot 10 \\
\overline{8} 28 \\
336\end{array}$ & $\begin{array}{l}\text { 1. } 2313 \\
\text { 1. } 2273 \\
\text { 1. } 2153 \\
\text { 1. } 2036 \\
\text { 1. } 1836\end{array}$ & $\begin{array}{l}2 \\
1 \\
1 \\
1 \\
1\end{array}$ \\
\hline $\begin{array}{c}716, \overline{10} \cdot 2 \cdot 4 \\
\overline{11} \cdot 1 \cdot 2 \\
0 \cdot 2 \cdot 10, \overline{7} \cdot 1 \cdot 11 \\
440\end{array}$ & $\begin{array}{l}\text { 1. } 1712 \\
\text { 1. } 1645 \\
\text { 1. } 1536 \\
\text { 1. } 1508 \\
\text { 1. } 1435\end{array}$ & $\begin{array}{r}2 \\
1 \\
1 \\
<1 \\
<1\end{array}$ \\
\hline$\overline{2} \cdot 0 \cdot 12$ & $\begin{array}{l}\text { 1. } 1387 \\
\text { 1. } 1335\end{array}$ & $<1$ \\
\hline
\end{tabular}

\section{References}

[1] W. H. Nebergall, J. C. Muhler, and H. G. Day, The preparation and properties of stannous fluoride, J. Am. Chem. Soc. 74, 1604 (1952).

[2] G. Bergerhoff, Zur Kristallstruktur des Zinn-IIfluorides, Acta Cryst. 15, 509 (1962). 
Powder data cards. None.

Additional published patterns. None.

NBS sample. The sample of strontium 1:1 borate was prepared at NBS by C. E. Weir. A suspension of hydrogen 3:1 borate (boric acid) and strontium carbonate was evaporated to dryness and heated at $1000{ }^{\circ} \mathrm{C}$. Spectrographic analysis showed the following major impurities: 0.01 to 0.1 percent each of barium and silicon; 0.001 to 0.01 percent each of calcium and sodium.

The sample is colorless and optically negative with $\mathrm{N}_{\alpha}=1.632, \mathrm{~N}_{\beta}=1.650$, and $\mathrm{N}_{\gamma}=1.660$.

The $d$-values of the three strongest lines are: $3.467,6.013$, and $2.688 \AA$.

Structural data. Block, Perloff, and Weir [1] in 1963 determined that strontium 1:1 borate has the space group $\mathrm{D}_{2 \mathrm{~h}}^{14}-\mathrm{Pnca}$ (No. 60) and $4\left(\mathrm{SrO} \cdot \mathrm{B}_{2} \mathrm{O}_{3}\right)$ per unit cell.

\section{Lattice constants}

\begin{tabular}{|c|c|c|c|c|}
\hline & & $a$ & $b$ & $c$ \\
\hline 1963 & $\begin{array}{l}\text { Block, Perloff } \\
\text { and Weir [1]. }\end{array}$ & $\begin{array}{c}\stackrel{\circ}{A} \\
6.577\end{array}$ & ${ }_{12 .}^{\stackrel{\circ}{A}} 02$ & $\begin{array}{c}\stackrel{\AA}{A} \\
\text { 4. } 329\end{array}$ \\
\hline 1963 & $\begin{array}{l}\text { National Bureau } \\
\text { of Standards } \\
\text { at } 25^{\circ} \mathrm{C} \text {. }\end{array}$ & $\begin{array}{r}6.5890 \\
\pm .0004\end{array}$ & $\begin{array}{l}12.018 \\
\pm .001\end{array}$ & $\begin{array}{r}4.3373 \\
\pm .0005\end{array}$ \\
\hline
\end{tabular}

The density of strontium 1:1 borate calculated from the NBS lattice constants is $3.350 \mathrm{~g} / \mathrm{cm}^{3}$ at $25^{\circ} \mathrm{C}$.

\section{Reference}

[1] S. Block, A. Perloff, and C. E. Weir. The crystallography of some $\mathrm{M}^{+2}$ borates, Acta Cryst. 17, 314 (1964).

\begin{tabular}{|c|c|c|}
\hline \multirow[t]{2}{*}{$h k l$} & \multicolumn{2}{|c|}{$\begin{array}{c}\text { Internal Standard, } \\
\text { Silver, } a=4.0861 \AA \\
\mathrm{Cu}, 1.5405 \AA \text { at } 25{ }^{\circ} \mathrm{C}\end{array}$} \\
\hline & $d$ & $I$ \\
\hline $\begin{array}{l}020 \\
111 \\
200 \\
210 \\
121\end{array}$ & $\begin{array}{ll} & \AA \\
\text { 6. } & 013 \\
\text { 3. } & 467 \\
\text { 3. } 292 \\
\text { 3. } 176 \\
\text { 3. } 101\end{array}$ & $\begin{array}{r}69 \\
100 \\
37 \\
47 \\
15\end{array}$ \\
\hline $\begin{array}{l}040 \\
220 \\
131 \\
141 \\
240\end{array}$ & $\begin{array}{l}\text { 3. } 004 \\
\text { 2. } 888 \\
\text { 2. } 688 \\
\text { 2. } 313 \\
\text { 2. } 221\end{array}$ & $\begin{array}{r}37 \\
40 \\
67 \\
5 \\
30\end{array}$ \\
\hline $\begin{array}{c}002 \\
022 \\
060,151 \\
241 \\
122\end{array}$ & $\begin{array}{l}\text { 2. } 169 \\
\text { 2. } 041 \\
\text { 2. } 003 \\
\text { 1. } 977 \\
\text { 1. } 948\end{array}$ & $\begin{array}{r}27 \\
21 \\
58 \\
9 \\
7\end{array}$ \\
\hline $\begin{array}{l}311 \\
202 \\
212 \\
331 \\
222\end{array}$ & $\begin{array}{l}\text { 1. } 934 \\
\text { 1. } 812 \\
\text { 1. } 792 \\
\text { 1. } 760 \\
\text { 1. } 734\end{array}$ & $\begin{array}{r}44 \\
8 \\
4 \\
23 \\
14\end{array}$ \\
\hline $\begin{array}{c}260 \\
400 \\
420 \\
171,242 \\
430 \\
351\end{array}$ & $\begin{array}{l}\text { 1. } 712 \\
\text { 1. } 647 \\
\text { 1. } 589 \\
\text { 1. } 5518 \\
\text { 1. } 5232 \\
\text { 1. } 5192\end{array}$ & $\begin{array}{r}10 \\
<2 \\
11 \\
21 \\
5\end{array}$ \\
\hline $\begin{array}{l}080 \\
062 \\
440 \\
113 \\
280\end{array}$ & $\begin{array}{l}\text { 1. } 5023 \\
\text { 1. } 4712 \\
\text { 1. } 4447 \\
\text { 1. } 4020 \\
\text { 1. } 3667\end{array}$ & $\begin{array}{l}4 \\
3 \\
5 \\
4 \\
5\end{array}$ \\
\hline $\begin{array}{l}262 \\
133 \\
371 \\
422 \\
460\end{array}$ & $\begin{array}{l}\text { 1. } 3434 \\
\text { 1. } 3320 \\
\text { 1. } 2911 \\
\text { 1. } 2820 \\
\text { 1. } 2724\end{array}$ & $\begin{array}{l}8 \\
8 \\
6 \\
6 \\
4\end{array}$ \\
\hline $\begin{array}{c}511 \\
153 \\
531 \\
282,333 \\
2 \cdot 10 \cdot 0\end{array}$ & $\begin{array}{l}\text { 1. } 2543 \\
\text { 1. } 2174 \\
\text { 1. } 2027 \\
\text { 1. } 1564 \\
\text { 1. } 1291\end{array}$ & $\begin{array}{r}9 \\
6 \\
15 \\
4 \\
<2\end{array}$ \\
\hline $\begin{array}{l}551 \\
480 \\
391 \\
600 \\
173\end{array}$ & $\begin{array}{l}\text { 1. } 1165 \\
\text { 1. } 1100 \\
\text { 1. } 1036 \\
\text { 1. } 0980 \\
\text { 1. } 0906\end{array}$ & $\begin{array}{l}5 \\
5 \\
5 \\
5 \\
3\end{array}$ \\
\hline $\begin{array}{c}620 \\
1 \cdot 11 \cdot 1 \\
640 \\
571 \\
2 \cdot 10 \cdot 2 \\
482,373\end{array}$ & $\begin{array}{l}\text { 1. } 0804 \\
\text { 1. } 0461 \\
\text { 1. } 0313 \\
\text { 1. } 0161 \\
\text { 1. } 0013 \\
\text { 0. } 9879\end{array}$ & $\begin{array}{r}5 \\
<2 \\
<2 \\
5 \\
3 \\
4\end{array}$ \\
\hline
\end{tabular}




\section{Terbium Arsenate, $\mathrm{TbAsO}_{4}$ (tetragonal)}

Powder data cards. None.

Additional published patterns. None.

NBS sample. The sample of terbium arsenate was prepared at NBS from a water solution of arsenic pentoxide and terbium trichloride. It was dried at $110{ }^{\circ} \mathrm{C}$. Spectrographic analysis showed the following major impurities: 0.01 to 0.1 percent silicon; 0.001 to 0.1 percent each of antimony, calcium, iron, magnesium, and lead.

The sample was colorless. The indices of refraction could not be determined because the sample was too fine.

The $d$-values of the three strongest lines are: $3.550,2.685$, and $1.834 \AA$.

Structural data. No reference to the structure of terbium arsenate was found, but it is apparently isostructural with yttrium arsenate with the space group $\mathrm{D}_{4 \mathrm{~h}}^{19}-\mathrm{I}_{1} / \mathrm{amd}(\mathrm{No} .141)$ and $4\left(\mathrm{TbAsO}_{4}\right)$ per unit cell.

\section{Lattice constants}

\begin{tabular}{|c|c|c|c|}
\hline & & $a$ & $c$ \\
\hline 1963 & $\begin{array}{l}\text { National Bureau of } \\
\text { Standards at } 25^{\circ} \mathrm{C} \text {. }\end{array}$ & 7. $\stackrel{\AA}{1025}$ & $\begin{array}{l}\stackrel{\AA}{A} \\
\text { 6. } 3536\end{array}$ \\
\hline
\end{tabular}

The density of terbium arsenate calculated from the NBS lattice constants is $6.172 \mathrm{~g} / \mathrm{cm}^{3}$ at $25^{\circ} \mathrm{C}$.

\begin{tabular}{|c|c|c|}
\hline \multirow[t]{2}{*}{$h k l$} & \multicolumn{2}{|c|}{$\begin{array}{l}\text { Internal Standard, } \\
\text { Tungsten, } a=3.1648 \AA \\
\text { Co, } 1.7889 \AA \text { at } 25^{\circ} \mathrm{C}\end{array}$} \\
\hline & $d$ & $I$ \\
\hline $\begin{array}{l}101 \\
200 \\
112 \\
220 \\
202\end{array}$ & 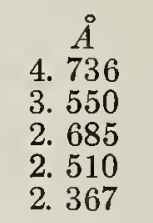 & $\begin{array}{r}8 \\
100 \\
69 \\
22 \\
7\end{array}$ \\
\hline $\begin{array}{l}301 \\
103 \\
321 \\
312 \\
400\end{array}$ & $\begin{array}{l}\text { 2. } 219 \\
\text { 2. } 029 \\
\text { 1. } 882 \\
\text { 1. } 834 \\
\text { 1. } 775\end{array}$ & $\begin{array}{r}9 \\
8 \\
6 \\
53 \\
16\end{array}$ \\
\hline $\begin{array}{l}420 \\
332 \\
204 \\
224 \\
512\end{array}$ & $\begin{array}{l}\text { 1. } 5878 \\
\text { 1. } 4809 \\
\text { 1. } 4500 \\
\text { 1. } 3423 \\
\text { 1. } 2755\end{array}$ & $\begin{array}{r}15 \\
14 \\
11 \\
8 \\
13\end{array}$ \\
\hline $\begin{array}{c}440 \\
600,404 \\
532 \\
424 \\
116\end{array}$ & $\begin{array}{l}\text { 1. } 2553 \\
\text { 1. } 1838 \\
\text { 1. } 1373 \\
\text { 1. } 1230 \\
\text { 1. } 0364\end{array}$ & $\begin{array}{r}4 \\
8 \\
8 \\
12 \\
4\end{array}$ \\
\hline $\begin{array}{l}444 \\
316 \\
604 \\
624\end{array}$ & $\begin{array}{r}0.9849 \\
.9577 \\
.9495 \\
.9168\end{array}$ & $\begin{array}{l}5 \\
7 \\
3 \\
5\end{array}$ \\
\hline
\end{tabular}

\section{Thallium Chromate, $\mathrm{Tl}_{2} \mathrm{CrO}_{4}$ (orthorhombic)}

Powder data cards. None.

Additional published patterns. Abbad and Rivoir [1] 1947.

NBS sample. The sample of thallium chromate was prepared at NBS from solutions of thallium nitrate and sodium chromate. Spectrographic analysis showed the following major impurities: 0.1 to 1.0 percent lead; 0.01 to 0.1 percent each of aluminum and silicon; and 0.001 to 0.01 percent each of barium, calcium, and antimony.

The color of the sample was yellow. The refractive indices could not be determined because the sample was too fine.

The $d$-values of the three strongest lines are: $3.145,3.060$, and $3.186 \AA$.

Structural data. Abbad and Rivoir [1] in 1947 determined that thallium chromate has the potassium sulfate structure, the space group $\mathrm{D}_{2 \mathrm{~h}}^{16}-\mathrm{Pnam}$ (No. 62), and $4\left(\mathrm{Tl}_{2} \mathrm{CrO}_{4}\right)$ per unit cell.
The lattice constants reported by Abbad and Rivoir have been converted from $\mathrm{kX}$ to angstrom units for comparison with the NBS values.

Lattice constants

\begin{tabular}{|c|c|c|c|c|}
\hline \multirow{5}{*}{$\begin{array}{l}1947 \\
1963\end{array}$} & \multirow{5}{*}{$\begin{array}{l}\text { Abbad and Rivoir } \\
{[1]_{\text {Non }}} \\
\text { National Bureau } \\
\text { of Standards } \\
\text { at } 25^{\circ} \mathrm{C}\end{array}$} & $a$ & $b$ & $c$ \\
\hline & & $\AA$ & $\AA$ & $\AA$ \\
\hline & & 782 & 10.70 & 502 \\
\hline & & 7. 908 & 10. 730 & 5. 913 \\
\hline & & \pm .001 & \pm .001 & \pm .001 \\
\hline
\end{tabular}

The density of thallium chromate calculated from the NBS lattice constants is $6.946 \mathrm{~g} / \mathrm{cm}^{3}$ at $25^{\circ} \mathrm{C}$. 
Thallium Chromate, $\mathrm{Tl}_{2} \mathrm{CrO}_{4}$ (orthorhombic)-Continued

\begin{tabular}{|c|c|c|}
\hline \multirow[t]{2}{*}{$h k l$} & \multicolumn{2}{|c|}{$\begin{array}{l}\text { Internal Standard, } \\
\text { Tungsten, } a=3.1648 \AA \\
\mathrm{Cu}, 1.5405 \AA \text { at } 25^{\circ} \mathrm{C}\end{array}$} \\
\hline & $d$ & $I$ \\
\hline $\begin{array}{l}020 \\
011 \\
120 \\
111 \\
210\end{array}$ & $\begin{array}{ll} & \AA \\
\text { 5. } & 37 \\
\text { 5. } 19 \\
\text { 4. } 45 \\
\text { 4. } 337 \\
\text { 3. } 714\end{array}$ & $\begin{array}{r}5 \\
8 \\
12 \\
23 \\
15\end{array}$ \\
\hline $\begin{array}{l}121 \\
201 \\
130 \\
220 \\
211\end{array}$ & $\begin{array}{l}3.553 \\
3.290 \\
3.263 \\
3.186 \\
3.145\end{array}$ & $\begin{array}{r}54 \\
27 \\
24 \\
57 \\
100\end{array}$ \\
\hline $\begin{array}{c}031 \\
002 \\
221 \\
040,112 \\
230\end{array}$ & $\begin{array}{l}3.060 \\
2.958 \\
2.805 \\
2.685 \\
2.654\end{array}$ & $\begin{array}{r}96 \\
53 \\
14 \\
6 \\
16\end{array}$ \\
\hline $\begin{array}{l}310 \\
140 \\
122 \\
320 \\
311\end{array}$ & $\begin{array}{l}\text { 2. } 561 \\
\text { 2. } 540 \\
\text { 2. } 461 \\
\text { 2. } 366 \\
\text { 2. } 349\end{array}$ & $\begin{array}{r}28 \\
25 \\
15 \\
8 \\
13\end{array}$ \\
\hline $\begin{array}{l}141 \\
212 \\
240 \\
321 \\
132\end{array}$ & $\begin{array}{l}\text { 2. } 336 \\
\text { 2. } 314 \\
\text { 2. } 221 \\
\text { 2. } 197 \\
\text { 2. } 191\end{array}$ & $\begin{array}{l}10 \\
10 \\
11 \\
15 \\
13\end{array}$ \\
\hline $\begin{array}{l}222 \\
330 \\
241 \\
150 \\
051\end{array}$ & $\begin{array}{ll}2 . & 167 \\
2 . & 122 \\
2 . & 079 \\
2 . & 071 \\
2 . & 017\end{array}$ & $\begin{array}{r}32 \\
9 \\
17 \\
15 \\
4\end{array}$ \\
\hline $\begin{array}{l}440 \\
232 \\
151 \\
312 \\
142\end{array}$ & $\begin{array}{l}\text { 1. } 977 \\
\text { 1. } 975 \\
\text { 1. } 954 \\
\text { 1. } 936 \\
\text { 1. } 9270\end{array}$ & $\begin{array}{r}19 \\
16 \\
6 \\
14 \\
13\end{array}$ \\
\hline $\begin{array}{c}250 \\
401 \\
420 \\
322,411 \\
123\end{array}$ & $\begin{array}{l}\text { 1. } 8863 \\
1.8750 \\
1.8553 \\
1.8472 \\
1.8013\end{array}$ & $\begin{array}{r}9 \\
12 \\
15 \\
14 \\
26\end{array}$ \\
\hline $\begin{array}{l}341 \\
060 \\
242 \\
203 \\
213\end{array}$ & $\begin{array}{l}\text { 1. } 7921 \\
\text { 1. } 7885 \\
\text { 1. } 7755 \\
\text { 1. } 7640 \\
\text { 1. } 7409\end{array}$ & $\begin{array}{r}26 \\
14 \\
7 \\
7 \\
17\end{array}$ \\
\hline
\end{tabular}

\begin{tabular}{|c|c|c|}
\hline \multirow[t]{2}{*}{$h k l$} & \multicolumn{2}{|c|}{$\begin{array}{l}\text { Internal Standard, } \\
\text { Tungsten, } a=3.1648 \AA \\
\mathrm{Cu}, 1.5405 \AA \text { at } 25^{\circ} \mathrm{C}\end{array}$} \\
\hline & $d$ & $I$ \\
\hline & $\AA$ & \\
\hline 430 & 1. 7305 & 22 \\
\hline 033 & 1. 7259 & 19 \\
\hline 332 & 1. 7235 & 14 \\
\hline 152 & 1. 6963 & 15 \\
\hline 161 & 1. 6726 & 13 \\
\hline 431 & 1. 6603 & 9 \\
\hline 402 & 1. 6434 & 5 \\
\hline 260 & 1. 6294 & 5 \\
\hline 412 & 1. 6250 & 5 \\
\hline 252 & 1. 5903 & 6 \\
\hline 422,261 & 1. 5713 & 10 \\
\hline 143 & 1. 5573 & 3 \\
\hline 062 & 1. 5299 & 6 \\
\hline 520 & 1. 5167 & 6 \\
\hline 511 & 1. 5125 & 6 \\
\hline 170 & 1. 5045 & 6 \\
\hline 432 & 1. 4931 & 8 \\
\hline 071 & 1. 4837 & 7 \\
\hline 004 & 1. 4782 & 5 \\
\hline 521 & 1. 4696 & 5 \\
\hline 450 & 1. 4539 & 4 \\
\hline 361 & 1. 4355 & 6 \\
\hline 262 & 1. 4271 & 5 \\
\hline 124,442 & 1. 4020 & 3 \\
\hline 403 & 1. 3959 & 4 \\
\hline 413 & 1. 3842 & 4 \\
\hline 540 & 1. 3623 & 6 \\
\hline 343 & 1. 3602 & 5 \\
\hline 522 & 1. 3498 & 5 \\
\hline 134 & 1. 3462 & 5 \\
\hline 080,172 & 1. 3410 & 6 \\
\hline 370 & 1. 3250 & 6 \\
\hline 600 & 1. 3182 & 4 \\
\hline
\end{tabular}

\section{Reference}

[1] M. Abbad and L. Rivoir, La estructura del cromato talioso, Anales Soc. españ. fís. y. quím 43, 831-836 (1947) 
Powder data cards. None.

Additional published patterns. None.

NBS sample. The sample of thulium arsenate was prepared at NBS from a water solution of arsenic pentoxide and thulium trichloride. It was dried at $110^{\circ} \mathrm{C}$. Spectrographic analysis showed the following major impurities: 0.01 to 0.1 percent each of silicon and antimony; 0.001 to 0.01 percent each of aluminum, calcium, iron, lead, and magnesium.

The sample was colorless. The indices of refraction could not be determined because the sample was too fine.

The $d$-values of the three strongest lines are: $3.497,2.644$, and $1.8064 \AA$.

Structural data. No reference to the structure of thulium arsenate was found, but it is apparently isostructural with yttrium arsenate with the space group $\mathrm{D}_{4 \mathrm{~h}}^{19}-\mathrm{I}_{1} /$ amd (No. 141) and $4\left(\mathrm{TmAsO}_{4}\right)$ per unit cell.

Lattice constants

\begin{tabular}{|c|c|c|c|}
\hline & & $a$ & $c$ \\
\hline 1963 & $\begin{array}{l}\text { National Bureau of } \\
\text { Standards at } 25^{\circ} \mathrm{C}\end{array}$ & $\begin{array}{r}\AA \\
\text { 6. } 9939 \\
\pm 0.0003\end{array}$ & $\begin{array}{r}\AA \\
6.2595 \\
\pm 0.0003\end{array}$ \\
\hline
\end{tabular}

The density of thulium arsenate calculated from the NBS lattice constants is $6.678 \mathrm{~g} / \mathrm{cm}^{3}$ at $25^{\circ} \mathrm{C}$.

\begin{tabular}{|c|c|c|}
\hline \multirow[t]{2}{*}{$h k l$} & \multicolumn{2}{|c|}{$\begin{array}{l}\text { Internal Standard, } \\
\text { Tungsten, } a=3.1648 \AA \\
\text { Cu, } 1.5405 \AA \text { at } 25{ }^{\circ} \mathrm{C}\end{array}$} \\
\hline & $d$ & $I$ \\
\hline $\begin{array}{l}101 \\
200 \\
211 \\
112 \\
220\end{array}$ & 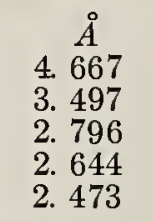 & $\begin{array}{r}11 \\
100 \\
5 \\
72 \\
23\end{array}$ \\
\hline $\begin{array}{l}202 \\
301 \\
103 \\
321 \\
312\end{array}$ & $\begin{array}{l}\text { 2. } 333 \\
\text { 2. } 1851 \\
\text { 1. } 9993 \\
\text { 1. } 8524 \\
\text { 1. } 8064\end{array}$ & $\begin{array}{r}5 \\
10 \\
7 \\
7 \\
55\end{array}$ \\
\hline $\begin{array}{l}400 \\
213 \\
420 \\
332 \\
204\end{array}$ & $\begin{array}{l}\text { 1. } 7483 \\
\text { 1. } 7354 \\
\text { 1. } 5638 \\
\text { 1. } 4584 \\
1.4281\end{array}$ & $\begin{array}{r}16 \\
3 \\
15 \\
15 \\
14\end{array}$ \\
\hline $\begin{array}{c}501 \\
224 \\
512 \\
440 \\
600,404\end{array}$ & $\begin{array}{l}\text { 1. } 3853 \\
\text { 1. } 3224 \\
\text { 1. } 2566 \\
\text { 1. } 2361 \\
\text { 1. } 1660\end{array}$ & $\begin{array}{r}3 \\
8 \\
11 \\
4 \\
7\end{array}$ \\
\hline $\begin{array}{c}532 \\
424,620 \\
116 \\
415,613 \\
444,640\end{array}$ & $\begin{array}{l}\text { 1. } 1199 \\
\text { 1. } 1060 \\
\text { 1. } 0207 \\
\text { 1. } 0073 \\
\text { 0. } 9701\end{array}$ & $\begin{array}{r}8 \\
12 \\
3 \\
<2 \\
5\end{array}$ \\
\hline $\begin{array}{c}316,552 \\
604 \\
624 \\
336,732 \\
800\end{array}$ & $\begin{array}{l}9433 \\
.9349 \\
.9032 \\
.8814 \\
.8743\end{array}$ & $\begin{array}{r}10 \\
2 \\
4 \\
5 \\
<2\end{array}$ \\
\hline $\begin{array}{l}217 \\
820 \\
516 \\
644 \\
536\end{array}$ & $\begin{array}{l}8597 \\
8480 \\
8304 \\
8245 \\
7872\end{array}$ & $\begin{array}{r}<2 \\
2 \\
5 \\
4 \\
5\end{array}$ \\
\hline
\end{tabular}


Titanium Dioxide, brookite, $\mathrm{TiO}_{2}$ (orthorhombic)

\section{Powder data cards}

\begin{tabular}{|c|c|c|}
\hline $\begin{array}{c}\text { Card } \\
\text { number }\end{array}$ & $\begin{array}{c}\text { Index } \\
\text { lines }\end{array}$ & Source \\
\cline { 2 - 3 } $3-0380$ & $\begin{array}{r}3.47 \\
2.90 \\
1.88\end{array}$ & British Museum. \\
\cline { 2 - 3 } & & \\
\hline
\end{tabular}

Additional published patterns. Sturdivant and Pauling [1].

NBS sample. The sample of brookite was bbtained from the U.S. National Museum. No. 97661 from Magnet Cove, Ark. Spectrographic analysis showed the following major impurities: 0.1 to 1.0 percent silicon; 0.01 to 0.1 percent each of aluminum, iron, and vanadium; 0.001 to 0.01 percent magnesium.

The color of the sample was black. The indices of refraction were not determined because they were higher than 2.00 .

The $d$-values of the three strongest lines are: $3.512,2.900$, and $3.465 \AA$.

Structural data. Sturdivant and Pauling [1] in 1928 determined that brookite has the space group $\mathrm{D}_{2 \mathrm{~L}}^{15}$-Pcab (No. 61) and $8\left(\mathrm{TiO}_{2}\right)$ per unit cell. Several lattice constants have been converted from $\mathrm{kX}$ to angstrom units for comparison with the NBS values.

\section{Lattice constants}

\begin{tabular}{|c|c|c|c|c|}
\hline & \multirow{2}{*}{$\begin{array}{l}\text { Sturdivant and } \\
\text { Pauling [1]. }\end{array}$} & $a$ & $b$ & $c$ \\
\hline 1928 & & $\stackrel{\stackrel{̊}{A}}{5.447}$ & $\begin{array}{c}\mathscr{A} \\
9.185\end{array}$ & $\begin{array}{l}\stackrel{\AA}{A} \\
\text { 5. } 145\end{array}$ \\
\hline 1928 & Schröder $[2]$ & 5. 450 & 9. 154 & 5. 163 \\
\hline 1932 & Phillips [3] $\ldots \ldots$ & 5.44 & 9.20 & 5. 14 \\
\hline 1963 & $\begin{array}{l}\text { National Bureau } \\
\text { of Standards } \\
\text { at } 25^{\circ} \mathrm{C} \text {. }\end{array}$ & $\begin{array}{l}{ }^{8} 5.4558 \\
\pm .0004 \\
\mathrm{~b} 5.456 \\
\pm .002\end{array}$ & $\begin{array}{l}9.1819 \\
\pm .0007 \\
9.174 \\
\pm .001\end{array}$ & $\begin{array}{l}\text { 5. } 1429 \\
\pm .0003 \\
5.138 \\
\pm .001\end{array}$ \\
\hline
\end{tabular}

a Sample described above.

b Sample from the National Museum No. R2108 from von der Söule, Virven, Tyrol. Spectrographic analysis showed the following major impurities: 0.1 to 1.0 percent iron; 0.01 to 0.1 percent each of magnesium, silicon, and vanadium; and 0.001 to 0.01 percent each of aluminum, barium, and manganese. The lattice constants given were derived from powder pattern data.

The density of brookite calculated from the NBS lattice constants (a) is $4.120 \mathrm{~g} / \mathrm{cm}^{3}$ at $25^{\circ} \mathrm{C}$.

\section{References}

[1] J. H. Sturdivant and L. Pauling, The crystal structure of brookite, Z. Krist. 68, 239 (1928).

[2] A. Schröder, Röntgenographische Feinbauuntersuchung am Brookit und über physikalische Eigenschaften der drei Titandioxyde, Z. Krist. 66, 493 (1928).

[3] F. C. Phillips, Crystals of brookite tabular parallel to the basal plane, Min. Mag. 23, 126-129 (1932).

\begin{tabular}{|c|c|c|}
\hline \multirow[t]{2}{*}{$h k l$} & \multicolumn{2}{|c|}{$\begin{array}{c}\text { Internal Standard, } \\
\text { Silver, } a=4.0861 \AA \\
\mathrm{Cu} \lambda=1.5405 \AA \text { at } 25^{\circ} \mathrm{C}\end{array}$} \\
\hline & $d$ & $I$ \\
\hline $\begin{array}{l}120 \\
111 \\
121 \\
200 \\
012\end{array}$ & $\begin{array}{c}\AA \\
3.512 \\
3.465 \\
2.900 \\
2.729 \\
2.476\end{array}$ & $\begin{array}{r}100 \\
79 \\
91 \\
4 \\
23\end{array}$ \\
\hline $\begin{array}{l}201 \\
131 \\
220 \\
211 \\
040\end{array}$ & $\begin{array}{l}\text { 2. } 409 \\
\text { 2. } 370 \\
\text { 2. } 344 \\
\text { 2. } 332 \\
\text { 2. } 296\end{array}$ & $\begin{array}{r}18 \\
6 \\
4 \\
4 \\
5\end{array}$ \\
\hline $\begin{array}{l}112 \\
022 \\
221 \\
032 \\
231\end{array}$ & $\begin{array}{l}\text { 2. } 254 \\
\text { 2. } 244 \\
\text { 2. } 133 \\
\text { 1. } 9685 \\
\text { 1. } 8934\end{array}$ & $\begin{array}{r}8 \\
18 \\
16 \\
16 \\
28\end{array}$ \\
\hline $\begin{array}{l}132 \\
212 \\
240 \\
320 \\
241\end{array}$ & $\begin{array}{l}\text { 1. } 8514 \\
\text { 1. } 8332 \\
1.7568 \\
\text { 1. } 6908 \\
1.6617\end{array}$ & $\begin{array}{r}18 \\
3 \\
3 \\
21 \\
28\end{array}$ \\
\hline $\begin{array}{l}151 \\
113 \\
232 \\
123 \\
052\end{array}$ & $\begin{array}{l}1.6486 \\
1.6098 \\
1.5968 \\
1.5408 \\
1.4942\end{array}$ & $\begin{array}{r}5 \\
13 \\
2 \\
7 \\
10\end{array}$ \\
\hline $\begin{array}{l}160 \\
312 \\
251 \\
203 \\
152\end{array}$ & $\begin{array}{l}1.4729 \\
\text { 1. } 4656 \\
\text { 1. } 4609 \\
1.4515 \\
1.4415\end{array}$ & $\begin{array}{r}4 \\
9 \\
12 \\
12 \\
6\end{array}$ \\
\hline $\begin{array}{l}213 \\
161 \\
400 \\
332 \\
401\end{array}$ & $\begin{array}{l}\text { 1. } 4336 \\
\text { 1. } 4167 \\
\text { 1. } 3640 \\
\text { 1. } 3358 \\
\text { 1. } 3186\end{array}$ & $\begin{array}{r}10 \\
9 \\
5 \\
8 \\
3\end{array}$ \\
\hline $\begin{array}{c}233 \\
004 \\
024,171 \\
431 \\
124\end{array}$ & $\begin{array}{l}\text { 1. } 3116 \\
\text { 1. } 2852 \\
\text { 1. } 2381 \\
\text { 1. } 2107 \\
\text { 1. } 2074\end{array}$ & $\begin{array}{r}2 \\
2 \\
10 \\
2 \\
1\end{array}$ \\
\hline $\begin{array}{c}333 \\
080 \\
441 \\
044 \\
521,423\end{array}$ & $\begin{array}{l}\text { 1. } 1552 \\
1.1480 \\
1.1432 \\
1.1217 \\
1.0399\end{array}$ & $\begin{array}{l}4 \\
2 \\
2 \\
4 \\
3\end{array}$ \\
\hline $\begin{array}{c}281 \\
324 \\
125 \\
372,254\end{array}$ & $\begin{array}{r}1.0366 \\
1.0237 \\
0.9873 \\
.9829\end{array}$ & $\begin{array}{l}2 \\
4 \\
2 \\
4\end{array}$ \\
\hline
\end{tabular}




\section{Zinc Telluride, ZnTe (cubic)}

\section{Powder data cards}

\begin{tabular}{|c|c|c|}
\hline $\begin{array}{c}\text { Card } \\
\text { number }\end{array}$ & $\begin{array}{c}\text { Index } \\
\text { lines }\end{array}$ & Source \\
\cline { 2 - 3 } $1-0582$ & $\begin{array}{r}3.50 \\
2.14 \\
1.83\end{array}$ & New Jersey Zinc Co. \\
\hline
\end{tabular}

Additional published patterns. None.

NBS sample. The sample of zinc telluride was obtained from Semi-Elements, Inc., Saxonburg, Pa. Spectrographic analysis showed the following major impurities: 0.01 to 0.1 percent of silicon, and 0.001 to 0.01 percent each of aluminum, barium, iron, and magnesium.

The color of the sample was reddish-brown. The indices of refraction were too high to be determined by the usual grain-immersion method.

The $d$-values of the three strongest lines are: $3.523,2.159$, and $1.840 \AA$.

Structural data. Zachariasen [1] in 1925 determined that zinc telluride has the zinc sulfide structure, the space group $\mathrm{T}_{\mathrm{d}}^{2}-\mathrm{F} 43 \mathrm{~m}$ (No. 216), and $4[\mathrm{ZnTe}]$ per unit cell. The unit cell measurement reported by Zachariasen has been converted from $\mathrm{kX}$ to angstrom units for comparison with the NBS value.

Lattice constants

\begin{tabular}{|c|c|c|}
\hline & & $\AA$ \\
\hline 1925 & Zachariasen $[1] \ldots \ldots$ & 6. 101 \\
\hline 1963 & $\begin{array}{l}\text { National Bureau of Standards } \\
\text { at } 25^{\circ} \mathrm{C}\end{array}$ & 6. 1026 \\
\hline
\end{tabular}

The density of zinc telluride calculated from the NBS lattice constant is $5.639 \mathrm{~g} / \mathrm{cm}^{3}$ at $25{ }^{\circ} \mathrm{C}$.

\begin{tabular}{|c|c|c|c|}
\hline \multirow[t]{2}{*}{$h k l$} & \multicolumn{3}{|c|}{$\begin{array}{l}\text { Internal Standard } \\
\text { Tungsten, } a=3.1648 \AA \\
\mathrm{Cu}, 1.5405 \AA \text { at } 25^{\circ} \mathrm{C}\end{array}$} \\
\hline & $d$ & $I$ & $a$ \\
\hline $\begin{array}{l}111 \\
200 \\
220 \\
311 \\
222\end{array}$ & 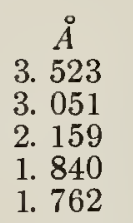 & $\begin{array}{r}100 \\
10 \\
81 \\
37 \\
3\end{array}$ & 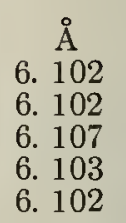 \\
\hline $\begin{array}{l}400 \\
331 \\
420 \\
422 \\
511\end{array}$ & $\begin{array}{l}\text { 1. } 526 \\
\text { 1. } 4003 \\
\text { 1. } 3645 \\
\text { 1. } 2456 \\
\text { 1. } 1745\end{array}$ & $\begin{array}{r}8 \\
13 \\
3 \\
10 \\
7\end{array}$ & $\begin{array}{l}\text { 6. } 103 \\
\text { 6. } 104 \\
\text { 6. } 102 \\
\text { 6. } 102 \\
\text { 6. } 103\end{array}$ \\
\hline $\begin{array}{l}440 \\
531 \\
600 \\
620 \\
533\end{array}$ & $\begin{array}{r}\text { 1. } 0789 \\
\text { 1. } 0315 \\
\text { 1. } 0171 \\
0.9648 \\
.9307\end{array}$ & $\begin{array}{l}3 \\
6 \\
1 \\
6 \\
3\end{array}$ & $\begin{array}{l}\text { 6. } 1028 \\
\text { 6. } 1042 \\
\text { 6. } 1026 \\
\text { 6. } 1022 \\
\text { 6. } 1028\end{array}$ \\
\hline $\begin{array}{l}622 \\
444 \\
711 \\
642 \\
731\end{array}$ & $\begin{array}{r}.9200 \\
.8808 \\
.8545 \\
.8155 \\
.7945\end{array}$ & $\begin{array}{l}1 \\
1 \\
6 \\
8 \\
6\end{array}$ & $\begin{array}{l}\text { 6. } 1026 \\
\text { 6. } 1026 \\
\text { 6. } 1024 \\
\text { 6. } 1026 \\
\text { 6. } 1026\end{array}$ \\
\hline \multicolumn{3}{|c|}{ Average value of last five lines } & 6. 1026 \\
\hline
\end{tabular}

\section{Reference}

[1] W. Zachariasen, The erystal structure of the tellurides of zinc, cadmium, and mercury, Norsk. geol Tidskrift 8, 302-306 (1925). 


\section{CUMULATIVE INDEX TO CIRCULAR 539, VOLUMES $1,2,3,4,5,6,7,8,9,10$, MONOGRAPH 25, SECTIONS 1, 2, AND $3^{5}$}

Aluminum, Al

Aluminum antimony, AlSb_..........

Aluminum calcium sulfate hydrate (ettringite), $\mathrm{Al}_{2} \mathrm{O}_{3} \cdot 6 \mathrm{CaO} \cdot 3 \mathrm{SO}_{3} \cdot 31 \mathrm{H}_{2} \mathrm{O}$ Aluminum chloride hexahydrate (chloraluminite), $\mathrm{AlCl}_{3} \cdot 6 \mathrm{H}_{2} \mathrm{O}$ Aluminum fluosilicate, topaz, $\mathrm{Al}_{2} \mathrm{SiO}_{4}(\mathrm{~F}, \mathrm{OH})$

Aluminum metaphosphate, $\mathrm{Al}\left(\mathrm{PO}_{3}\right)_{3} \ldots \ldots$ Aluminium or thophosphate (berlinite),

$\mathrm{AlPO}_{4}$ (trigonal) rhombic) - . . . . . Aluminum oxide, (corundum), alpha $\mathrm{Al}_{2} \mathrm{O}_{3}--$

Aluminum oxide monohydrate (böhmite), alpha $\mathrm{Al}_{2} \mathrm{O}_{3} \cdot \mathrm{H}_{2} \mathrm{O}$ beta $\mathrm{Al}_{2} \mathrm{O}_{3} \cdot \mathrm{H}_{2} \mathrm{O}$
Aluminum $3: 2$

Aluminum $3: 2$ silicate (mullite), Ammonium aluminum sulfate dodecahydrate (tes chermigite), $\mathrm{NH}_{4} \mathrm{Al}\left(\mathrm{SO}_{4}\right)_{2} \cdot 12 \mathrm{H}_{2} \mathrm{O}_{-}$

Ammonium azide, $\mathrm{NH}_{4} \mathrm{~N}_{3}$

Ammonium bicarbonate (teschemacherite), $\left(\mathrm{NH}_{4}\right) \mathrm{HCO}_{3 \ldots} \ldots$

Ammonium bromide, $\left.\mathrm{NH}_{4} \mathrm{Br}_{1} \mathrm{NH}_{4}\right) \mathrm{Os}_{6} \mathrm{Br}_{6}$ Ammonium bromoosmate, $\left(\mathrm{NH}_{4}\right) \mathrm{O}_{5} \mathrm{Br}_{6--.-}$ Ammonium bromoselenate, $\left(\mathrm{NH}_{4}\right)_{2} \mathrm{SeBr}_{6-. .-}$ Ammonium bromotellurate, $\left(\mathrm{NH}_{4}\right)_{2} \mathrm{TeBr}_{6-.-}$ Ammonium chloride (sal-ammoniac),

$\mathrm{NH}_{4} \mathrm{Cl} \ldots$

Ammonium chloroosmate, $\left(\mathrm{NH}_{4}\right)_{2} \mathrm{OsCl}_{6}-\mathrm{Cl}_{6}$

Ammonium chloropalladate, $\left(\mathrm{NH}_{4}\right)_{2} \mathrm{PdCl}_{6---}$ Ammonium chloropalladite, $\left(\mathrm{NH}_{4}\right)_{2} \mathrm{PdCl}_{4---}$ Ammonium chloroplatinate, $\left(\mathrm{NH}_{4}\right)_{2} \mathrm{PtCl}_{6-}-$ Ammonium chlorostannate $\left(\mathrm{NH}_{4}\right)_{2} \mathrm{SnCl}_{6-.--}$ Ammonium chlorotellurate, $\left(\mathrm{NH}_{4}\right)_{2} \mathrm{Te} \mathrm{Cl}_{6---}$ Ammonium chromium sulfate dodecahydrate, $\mathrm{NH}_{4} \mathrm{Cr}\left(\mathrm{SO}_{4}\right)_{2} \cdot 12 \mathrm{H}_{2} \mathrm{O}$ A mmonium dihydrogen phosphate, $\mathrm{NH}_{4} \mathrm{H}_{2} \mathrm{PO}_{4}$ Ammonium fluoberyllate, $\left(\mathrm{NH}_{4}\right)_{2} \mathrm{BeF}_{4-. .-}$ Ammonium fluoborate, $\mathrm{NH}_{4} \mathrm{BF}_{4-}$ Ammonium fluogermanate, ( $\left.\mathrm{NH}_{4}\right)_{2} \mathrm{GeF}_{6}-1-$ -

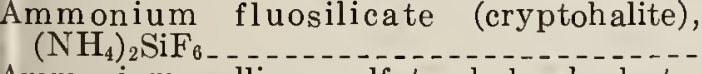
Ammonium gallium sulfate dodecahydrate, $\mathrm{NH}_{4} \mathrm{Ga}\left(\mathrm{SO}_{4}\right)_{2} \cdot 12 \mathrm{H}_{2} \mathrm{O}$

Ammonium iodide, $\mathrm{NH}_{4} \mathrm{I}_{\ldots} \ldots$ Ammonium iron sulfate dodecahydrate, $\mathrm{NH}_{4} \mathrm{Fe}\left(\mathrm{SO}_{4}\right)_{2} \cdot 12 \mathrm{H}_{2} \mathrm{O} \ldots$ Ammonium metavanadate, $\mathrm{NH}_{4} \mathrm{~V} \mathrm{O}_{3}-\ldots-1$ Ammonium nitrate (ammonia-niter), Ammonium oxalate monohydrate (oxammite), $\left(\mathrm{NH}_{4}\right)_{2} \mathrm{C}_{2} \mathrm{O}_{4} \cdot \mathrm{H}_{2} \mathrm{O}_{-}-\mathrm{NH}_{4} \mathrm{ClO}_{4}$, (orthoAmmonium perchlorate, $\mathrm{NH}_{4} \mathrm{ClO}_{4}$, (orthoAmmonium perrhenate, $\mathrm{NH}_{4} \mathrm{ReO}_{4}$ Ammonium phosphomolybdate tetrahydrate, $\left(\mathrm{NH}_{4}\right)_{3} \mathrm{PO}_{4}\left(\mathrm{MoO}_{3}\right)_{12} \cdot 4 \mathrm{H}_{2} \mathrm{O}-\overline{\left(\mathrm{NH}_{4}\right)_{2} \mathrm{SO}_{4}}$ (revised) Ammonium zirconium fluoride, $\left(\mathrm{NH}_{4}\right)_{3} \mathrm{Zr}_{\mathrm{r}} \mathrm{F}_{7-}$ Antimony, $\mathrm{Sb}_{-}$

Frrther work on this program is in progress, and it is anticipated that additional sections will be issi ed. Therefore, the accumulative index here isnot necessarily the concluding index for the project.

m-Monograph 25.

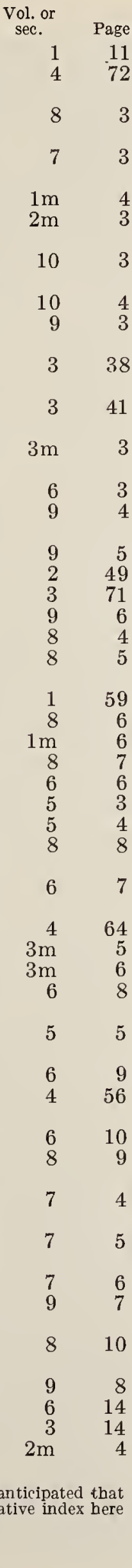

Vol. or sec.

Antimony (III) iodide, $\mathrm{SbI}_{3}$
Antimony (III) oxide (senarmontite), $\mathrm{Sb}_{2} \mathrm{O}_{3}$

(cubic)
(orthorh (III) oxide, valentinite, $\mathrm{Sb}_{2} \mathrm{O}_{3}$ Antimony (IV) oxide (cervantite), $\mathrm{Sb}_{2} \mathrm{O}_{4--}$ Antimony $(V)$ Oxide, $\mathrm{Sb}_{2} \mathrm{O}_{5} \ldots \ldots \ldots$ Antimony selenide, $\mathrm{Sb}_{2} \mathrm{Se}_{3}$ Antimony telluride, $\mathrm{Sb}_{2} \mathrm{~T}_{3}$

Arsenic, As

Arsenic (III) iodide, $\mathrm{AsI}_{3}$

Arsenic trioxide, claudetite, $\mathrm{As}_{2} \mathrm{O}_{3}$ (monoclinic)

Arsenic trioxide (arsenolite), $\mathrm{A}_{S_{2}} \mathrm{O}_{3}$ (cubic) - -

$\begin{array}{ll}\text { Arsenic trioxide (arsenolite), } \mathrm{A}_{S_{2}} \mathrm{O}_{3} \text { (cubic) _. } & 1 \\ \text { Barium, } \mathrm{Ba} & \end{array}$

Barium arsenate, $\mathrm{Ba}_{3}\left(\mathrm{AsO}_{4}\right)_{2}$

Barium bromide monohydrate, $\mathrm{BaBr}_{2} \cdot \overline{\mathrm{H}}_{2} \mathrm{O}_{--}^{--}$

Barium carbonate (witherite), $\mathrm{BaCO}_{3}$ (or thorhombic)

Barium carbonate, $\mathrm{BaCO}_{3}$ (cubic) at 1075

Barium fluoride, $\mathrm{BaF}_{2-}$

Barium molybdate, $\mathrm{Ba} \mathrm{MoO}_{\mathrm{O}}$

Barium molybdate, $\mathrm{Ba} \mathrm{MoO}_{4}-\overline{\mathrm{Ba}}\left(\mathrm{NO}_{3}\right)_{2 \ldots} \ldots$

Barium perchlorate trihydrate, $\mathrm{Ba}\left(\mathrm{ClO}_{4}\right)_{2} \cdot 3 \mathrm{H}_{2} \mathrm{O}_{-}$

Barium peroxide, $\mathrm{BaO}_{2-}$

Barium stannate, $\mathrm{BaSn} \mathrm{O}_{3}$

Barium sulfate (barite), $\mathrm{BaSO}$

Barium sulfide, $\mathrm{BaS}$

Barium titanate, $\mathrm{BaTiO}_{3}$

Barium tungstate, $\mathrm{BaWO}$

Barium zirconate, ${\mathrm{BaZ} \mathrm{rO}_{3}}$

Beryllium aluminum oxide (chrysoberyl), $\mathrm{BeAl}_{2} \mathrm{O}_{4}$

Beryllium aluminum silicate, beryl,

$\mathrm{Be}_{3} \mathrm{Al}_{2}\left(\mathrm{SiO}_{3}\right)_{6}$

Beryllium chromium oxide, $\mathrm{BeCr}_{2} \mathrm{O}_{4 \ldots} \ldots .$.

Beryllium germanate, $\mathrm{Be}_{2} \mathrm{GeO}_{4}$

Beryllium orthosilicate, phenacite, $\mathrm{Be}_{2} \mathrm{SiO}_{4--}$

Beryllium oxide (bromellite), $\mathrm{BeO}$

Bismuth, Bi

Bismuth fluoride, $\mathrm{BiF}_{3}$

Bismuth (III) iodide, $\mathrm{BiI}_{3} \ldots$

Bismuth orthophosphate, $\mathrm{BiPO}_{4}$ (monoclinic)

$\mathrm{Bismuth}$ orthovanadate, low form, $\mathrm{BiVO}_{4}$ (tetragonal)

Bismuth orthovanadate, high form, $\mathrm{BiVO}$ (monoclinic)

Bismuth oxybromide, $\mathrm{BiOBr}$

Bismuth oxychloride (bismoclite), $\mathrm{BiO} \mathrm{Cl}_{---}$

Bismuth oxyiodide, BiOI

Bismuth sulfide (bismuthinite), $\mathrm{Bi}_{2} \mathrm{~S}_{3 \ldots} \ldots$

Bismuth telluride (tellurobismuthite), $\mathrm{Bi}_{2} \mathrm{Te}_{3}$

Bismuth trioxide (bismite), alpha $\mathrm{Bi}_{2} \mathrm{O}_{3} \ldots$

Cadmium, Cd

Cadmium bromide, $\mathrm{CdBr}$

Cadmium carbonate (otavite), $\mathrm{Cd} \mathrm{CO}_{3-\ldots}$

Cadmium chloride, $\mathrm{CdCl}_{2 \ldots} \ldots$

Cadmium cyanide, $\mathrm{Cd}(\mathrm{CN})_{2} \ldots$

Cadmium molybdate, $\mathrm{CdMOO}_{4}$

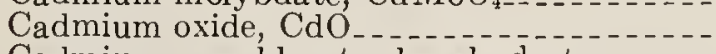

Cadmium perchlorate hexahydrate, $\mathrm{Cd}\left(\mathrm{ClO}_{4}\right)_{2} \cdot 6 \mathrm{H}_{2} \mathrm{O}$

Cadmium selenide, $\mathrm{CdSe}$, (hexagonal)

Cadmium sulfate, $\mathrm{CdSO}_{4}$

Cadmium sulfide (greenockite), $\mathrm{CdS}_{-} \ldots$

Cadmium telluride, CdTe.

Cadmium tungstate, $\mathrm{CdW} \mathrm{O}_{4}$

\section{6}

10

10

10

$3 \mathrm{~m}$

$3 \mathrm{~m}$

3

6

$3 m$

$2 \mathrm{~m}$

2

10

7

1

$2 \mathrm{~m}$

6

$3 \mathrm{~m}$

3

7
Page

31

6

8
10

7

6

6

17

9

51

6

10

54

11

70

81

7

18

11

65

45

9

10

13

12

13

11

36

20

20

11

13

14

14

14

54

16

23

16

17

10

17

11

18

8
21

27

19

12

20

15

21 

MONOGRAPH 25, SECTIONS 1, 2, AND $3^{5}$-Continued

\begin{tabular}{|c|c|c|c|c|}
\hline $\begin{array}{l}\text { Vol. or } \\
\text { sec. }\end{array}$ & Page & & $\begin{array}{l}\text { Vol. or } \\
\text { sec. }\end{array}$ & Page \\
\hline 5 & 10 & Chromium orthophosphate, beta, $\mathrm{CrPO}_{4-\ldots}$ & 9 & 26 \\
\hline 9 & 20 & $\begin{array}{l}\text { Chromium (III) oxide, } \mathrm{Cr}_{2} \mathrm{O}_{3 \ldots} \ldots \\
\text { Chromium silicide, } \mathrm{Cr}_{3} \mathrm{Si}_{\ldots} \ldots \ldots\end{array}$ & $\begin{array}{l}5 \\
6\end{array}$ & $\begin{array}{l}22 \\
29\end{array}$ \\
\hline 10 & 15 & Cobalt aluminum oxide, $\mathrm{CoAl}_{2} \mathrm{O}_{4-}$ & 9 & 27 \\
\hline 8 & 15 & $\begin{array}{l}\text { Cobalt arsenide (skutterudite), CoAs } \\
\text { Cobalt(II) carbonate (spherocobaltite), }\end{array}$ & 10 & 21 \\
\hline 3 & 53 & $\mathrm{CoCO}_{3}$ & 10 & 24 \\
\hline 2 & 51 & $\begin{array}{l}\text { Cobalt diarsenide, } \mathrm{CoAs}_{2} \\
\text { Cobaltfluosilicate hexahydrate, } \mathrm{CoSiF}_{6} \cdot 6 \mathrm{H}_{2} \mathrm{O}_{-}\end{array}$ & $\begin{array}{r}10 \\
3 \mathrm{~m}\end{array}$ & $\begin{array}{l}26 \\
27\end{array}$ \\
\hline 7 & 13 & $\begin{array}{l}\text { Cobalt gallate, } \mathrm{CoG} a_{2} \mathrm{O}_{4} \\
\text { Cobalt germanate, } \mathrm{Co}_{2} \mathrm{GeO}_{4}\end{array}$ & $\begin{array}{l}10 \\
10\end{array}$ & $\begin{array}{l}27 \\
27\end{array}$ \\
\hline 10 & 16 & $\begin{array}{l}\text { Cobalt iron arsenide (safflorite), } \mathrm{CoFeAs} \\
\text { Cobalt mercury thioc yanate, } \mathrm{Co}\left[\mathrm{Hg}(\mathrm{CNS})_{4}\right]_{-}\end{array}$ & $\begin{array}{r}10 \\
2 \mathrm{~m}\end{array}$ & $\begin{array}{l}28 \\
13\end{array}$ \\
\hline 10 & 17 & Cobalt(II) oxide, $\mathrm{CoO}$ & 9 & 28 \\
\hline 1 & 69 & $\begin{array}{l}\text { Cobalt(II, III) oxide, } \mathrm{Co}_{3} \mathrm{O}_{4} \\
\text { Cobalt perchlorate hexahydrate, } \mathrm{Co}\left(\mathrm{ClO}_{4}\right)_{2}\end{array}$ & 9 & 29 \\
\hline $3 \mathrm{~m}$ & 22 & $6 \mathrm{H}_{2} \mathrm{O}$ & $3 \mathrm{~m}$ & 28 \\
\hline $\begin{array}{r}8 \\
10\end{array}$ & $\begin{array}{l}16 \\
18\end{array}$ & Cobalt sulfate, beta, $\mathrm{CoSO}_{4-}$ & $2 \mathrm{~m}$ & 14 \\
\hline $\begin{array}{r}10 \\
1\end{array}$ & $\begin{array}{l}18 \\
58\end{array}$ & $\begin{array}{l}\text { Copper, Cu}+ \\
\text { Copper(I) bromide, } \mathrm{Cu} \mathrm{Br}_{-}\end{array}$ & $\begin{array}{l}1 \\
4\end{array}$ & $\begin{array}{l}15 \\
36\end{array}$ \\
\hline 10 & 19 & $\begin{array}{l}\mathrm{C} o p p e r \\
\left(\mathrm{CO}_{3}\right)_{2}\end{array}$ & 10 & 30 \\
\hline 9 & 22 & Copper carbonate, basic, (malachite), & & \\
\hline $\begin{array}{l}6 \\
7\end{array}$ & $\begin{array}{l}22 \\
14\end{array}$ & Copper(I) chloride (nantokite), $\mathrm{CuCl}$ & $\begin{array}{r}10 \\
4\end{array}$ & $\begin{array}{l}31 \\
35\end{array}$ \\
\hline 1 & 43 & Copper(I) iodide (marshite), CuI & 4 & 38 \\
\hline 4 & 65 & Copper(I) oxide (cuprite), $\mathrm{Cu}_{2} \mathrm{O}$ & 2 & 23 \\
\hline 7 & 15 & Copper(II) oxide (tenorite), C & 1 & 49 \\
\hline 6 & 23 & Copper sulfate (chalcocyanite) & $3 \mathrm{~m}$ & 29 \\
\hline 2 & 5 & Copper(II) sulfide (covellite), & 4 & 13 \\
\hline $1 \mathrm{~m}$ & 8 & Dysprosium arsenate, $\mathrm{DyAsO}_{4}$ & $3 \mathrm{~m}$ & 30 \\
\hline 8 & 17 & $\begin{array}{l}\text { Dysprosium gallium oxide } 3: 5 \text {, } \\
\text { Dy }_{3} \mathrm{Ga}_{2}\left(\mathrm{GaO}_{4}\right)_{3}\end{array}$ & $2 \mathrm{~m}$ & \\
\hline 10 & 20 & Dysprosium sesquioxide, $\mathrm{Dy}_{2} \mathrm{O}_{3} \ldots$ & & 30 \\
\hline $3 \mathrm{~m}$ & 24 & $\begin{array}{l}\text { Erbium arsen ate, } \mathrm{ErAsO}_{4-} \\
\text { Erbium gallium oxide } 3: 5 \\
\mathrm{Er}\end{array}$ & $\begin{array}{l}3 \mathrm{~m} \\
1 \mathrm{~m}\end{array}$ & 31 \\
\hline 1 & 56 & $\begin{array}{l}\text { Erblum gallum oxide } 3: 5, \mathrm{Er}_{3} \mathrm{C} \\
\text { Erbium manganite, ErMnO }\end{array}$ & $2 \mathrm{~m}$ & $\begin{array}{l}12 \\
16\end{array}$ \\
\hline $1 \mathrm{~m}$ & 9 & $\begin{array}{l}\text { Erbium phosphate, } \mathrm{ErPO}_{4} \mathrm{PO}_{2} \\
\text { Erbium sesquioxide, } \mathrm{E}_{2} \mathrm{O}_{3-}\end{array}$ & $\begin{array}{l}9 \\
8\end{array}$ & $\begin{array}{l}31 \\
25\end{array}$ \\
\hline 6 & 25 & Europium arsenate, $\mathrm{EuAs}_{4} \mathrm{O}_{4}$ & $3 \mathrm{~m}$ & 32 \\
\hline 8 & 18 & Europium (III) chloride, $\mathrm{EuCl}_{3-}$ & $1 \mathrm{~m}$ & 13 \\
\hline 3 & 49 & Europium gallium oxide $3: 5, \mathrm{Eu}_{3} \mathrm{Ga}_{2}\left(\mathrm{GaO}_{4}\right)_{3-}$ & $2 \mathrm{~m}$ & 17 \\
\hline $2 \mathrm{~m}$ & 10 & Europium ox ychloride, EuOCl $\ldots \ldots$ & $1 \mathrm{~m}$ & 13 \\
\hline 8 & 19 & Gadolinium fluoride, $\mathrm{GdF}_{3} \ldots$ & $1 \mathrm{~m}$ & 14 \\
\hline 8 & 20 & Gadolinium gallium oxide $3: 5$, & & \\
\hline 9 & 24 & $\mathrm{Gd}_{3} \mathrm{Ga}_{2}\left(\mathrm{GaO}_{4}\right)_{3} \ldots$ & $2 \mathrm{~m}$ & \\
\hline 8 & 20 & Gadolinium oxide, $\mathrm{Gd}_{2} \mathrm{O}_{3}$ & $1 \mathrm{~m}$ & 16 \\
\hline 2 & 44 & Gadoiinium ox ychloride, $\mathrm{GdOCl}$ & $1 \mathrm{~m}$ & 17 \\
\hline $2 \mathrm{~m}$ & 11 & Gallium, Ga $\ldots \ldots$ & 2 & 9 \\
\hline 5 & 14 & Gallium arsenide, GaAs .... & $3 \mathrm{~m}$ & \\
\hline 5 & 16 & Gallium antimonide, $\mathrm{GaSb}$ & 6 & 30 \\
\hline $3 \mathrm{~m}$ & 25 & $\begin{array}{l}\text { Gallium oxide, alpha, } \mathrm{Ga}_{2} \mathrm{O}_{3} \\
\text { Gallium phosphate ( } \alpha \text {-quartz type), } \mathrm{GaPO}_{4-}\end{array}$ & $\begin{array}{l}4 \\
8\end{array}$ & $\begin{array}{l}25 \\
27\end{array}$ \\
\hline $\begin{array}{l}8 \\
3\end{array}$ & 21 & Germanium, Ge $\ldots$ & 1 & 18 \\
\hline $\begin{array}{l}3 \\
8\end{array}$ & $\begin{array}{l}50 \\
22\end{array}$ & $\begin{array}{l}\text { Germanium dioxide, } \mathrm{GeO}_{2} \text { (hexagonal) } \\
\text { (low form) }\end{array}$ & 1 & 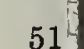 \\
\hline 5 & 17 & Germanium dioxide, $\mathrm{GeO}_{2}$ (tetragonal) & & \\
\hline 6 & 27 & (high form) & 8 & 28 \\
\hline $3 \mathrm{~m}$ & 26 & Germanium(IV) iodide, $\mathrm{GeI}_{4-.}$ & 5 & 25 \\
\hline 5 & 19 & $\begin{array}{l}\text { Gold, Au } \\
\text { Gold antimony } 1: 2 \text { (aurostibite), } \mathrm{AuSb}_{2}\end{array}$ & $\frac{1}{7}$ & $\begin{array}{l}33 \\
18\end{array}$ \\
\hline 8 & 23 & Gold(I) cyanide, AuCN & 10 & 33 \\
\hline 4 & 47 & $\begin{array}{l}\text { Gold tin, } 1: 1 \text { AuSn } \\
\text { Hafnium, Hf }\end{array}$ & $\begin{array}{l}7 \\
3\end{array}$ & $\begin{array}{l}19 \\
18\end{array}$ \\
\hline 6 & 28 & Holmium arsenate, $\mathrm{H}_{0} \mathrm{As}_{4}$ & $3 \mathrm{~m}$ & 34 \\
\hline 10 & 25 & Holmium ethylsulfate nonahydrate, & & 104 \\
\hline $1 \mathrm{~m}$ & 10 & $\mathrm{Ho}\left[\left(\mathrm{C}_{2} \mathrm{H}\right)_{5} \mathrm{SO}_{4}\right]_{3} \cdot 9 \mathrm{H}_{2} \mathrm{O} \ldots$ & $\operatorname{lm}_{9}$ & 18 \\
\hline 7 & 17 & $\begin{array}{l}\mathrm{H} \text { Holmium sesquioxide, } \mathrm{H}_{\mathrm{O}_{2}} \mathrm{O}_{3} \\
\text { Indium, In }\end{array}$ & 3 & $\begin{array}{l}32 \\
12\end{array}$ \\
\hline $1 \mathrm{~m}$ & 11 & $\operatorname{InSb} b_{\ldots} \ldots \ldots$ & 4 & 73. \\
\hline $\begin{array}{r}5 \\
2 \mathrm{~m}\end{array}$ & $\begin{array}{l}20 \\
12\end{array}$ & $\begin{array}{l}\text { Indium arsenide, } \mathrm{In} A \mathrm{As} \ldots \ldots \\
\text { Indium oxide, } \operatorname{In}_{2} \mathrm{O}_{3} \ldots \ldots\end{array}$ & $\begin{array}{r}3 \mathrm{~m} \\
5\end{array}$ & $\begin{array}{ll}30 \\
26\end{array}$ \\
\hline & & Indium phosphate, $\mathrm{In}_{\mathrm{P}} \mathrm{PO}_{4}$ & 8 & 291 \\
\hline
\end{tabular}

tri-Calcium aluminate, $3 \mathrm{CaO} \cdot \mathrm{Al}_{2} \mathrm{O}_{3}$
Calcium aluminate $12: 7,12 \mathrm{CaO} \cdot 7 \mathrm{Al}_{2} \mathrm{O}_{3} \ldots$

Calcium aluminum germanate,

$\mathrm{Ca}_{3} \mathrm{Al}_{2}\left(\mathrm{GeO}_{4}\right)_{3}$

Calcium carbonate (aragonite), $\mathrm{CaCO}_{3}$ (orthorhombic) -

Calcium carbonate (calcite) $\mathrm{CaCO}_{3}$ (hexagonal)

Calcium chromate, $\mathrm{Ca} \mathrm{CrO}_{4}$

Calcium chromium germanate, $\mathrm{Ca}_{3} \mathrm{Cr}_{2}\left(\mathrm{GeO}_{4}\right)_{3}$

Calcium chromium silicate (uvarovite), $\mathrm{Ca}_{3} \mathrm{Cr}_{2}\left(\mathrm{SiO}_{4}\right)_{3}$

Calcium fluoride (fluorite), $\mathrm{CaF}_{2}$

Calcium fluoride phosphate (fluorapatite), $\mathrm{Ca} 5 \mathrm{~F}\left(\mathrm{PO}_{4}\right)_{3} \ldots$

Calcium formate, $\left.\mathrm{Ca}(\mathrm{HCO})_{2}\right)_{2} \mathrm{Ca}_{3} \mathrm{Ga}_{2}\left(\mathrm{GeO}_{4}\right)_{3-}$

Calcium hydroxide (portlandite), $\mathrm{C}_{2}(\mathrm{OH})_{2--}$

Calcium iron germanate, $\mathrm{Ca}_{3} \mathrm{Fe}_{2}\left(\mathrm{GeO}_{4}\right)_{3} \ldots$

Calcium iron silicate (andradite),

$\mathrm{Ca}_{3} \mathrm{Fe}_{2} \mathrm{Si}_{3} \mathrm{O}_{12}$
Calcium molybdate (powellite), $\mathrm{CaMoO} \mathrm{M}_{4-}$

Calcium nitrate, $\mathrm{Ca}\left(\mathrm{NO}_{3}\right)_{2 \ldots} \ldots$

Calcium oxide, $\mathrm{CaO}$

Calcium sulfate (anhydrite), $\mathrm{CaSO}_{4 \ldots . . .}$

Calcium sulfide (oldhamite), CaS

Calcium tungstate scheelite, $\mathrm{CaWO}_{4}$

Carbon, diamond, $\mathrm{C}$

Cerium (III) chloride, $\mathrm{CeCl}_{3}$

Cerium (III) fluoride, $\mathrm{CeF}_{3}$

Cerium, magnesium nitrate 24 -hydrate, $\mathrm{Ce}_{2} \mathrm{Mg}_{3}\left(\mathrm{NO}_{3}\right)_{12} \cdot 24 \mathrm{H}_{2} \mathrm{O}$

Cerium niobium titanium oxide (eschynite), $\mathrm{CeNbTiO}_{6}$

Cerium (IV) oxide (cerianite) $\mathrm{CeO}_{2 . . . .}$

Cerium (III) vanadate, $\mathrm{CeVO}_{4}$

Cesium aluminum sulfate dodecahydrate, $\mathrm{CsAl}\left(\mathrm{SO}_{4}\right)_{2} \cdot 12 \mathrm{H}_{2} \mathrm{O}$

Cesium bromate, $\mathrm{Cs}_{\mathrm{BrO}} \mathrm{Br}_{3 .} \ldots$

Cesium bromide, $\mathrm{CsBr}$

Cesium bromoosmate (IV), $\mathrm{C}_{2} \mathrm{O}_{\mathrm{S}} \mathrm{Br}_{6-\ldots}$

Cesium bromoplatinate, $\mathrm{C}_{\mathrm{S}_{2}} \mathrm{PtBr}_{6} \ldots$

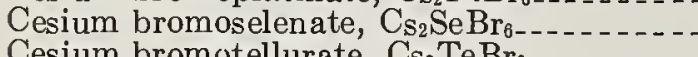

Cesium bromotellurate, $\mathrm{C}_{2} \mathrm{TeBr}_{6} \ldots$

Cesium chlorate, $\mathrm{CsClO}_{3-}$

Cesium chloride, $\mathrm{CsCl}_{\mathrm{S}}$

Cesium chloroosmate (IV), $\mathrm{CS}_{2} \mathrm{Os}_{\mathrm{S}} \mathrm{Cl}_{6} \ldots$

Cesium chloroplatinate, $\mathrm{C}_{\mathrm{S}_{2}} \mathrm{PtCl}_{6}$

Cesium chlorostannate, $\mathrm{C}_{2} \mathrm{SnCl}_{6} \ldots$

Cesium chromate, $\mathrm{C}_{\mathrm{S}_{2}} \mathrm{CrO}_{4}$

Cesium chromium sulfate dodecahydrate, $\mathrm{Cs} \mathrm{Cr}\left(\mathrm{SO}_{4}\right)_{2} \cdot 12 \mathrm{H}_{2} \mathrm{O}$

Cesium dichloroiodide, $\mathrm{C}_{\mathrm{SI}} \mathrm{Cl}_{2 .} \ldots$

Cesium fluoborate, $\mathrm{CSBF}_{4}$

Cesium fluogermanate, $\mathrm{Cs}_{2} \mathrm{GeF}_{6} \ldots$

Cesium fluoplatinate, $\mathrm{C}_{2} \mathrm{PtF}_{6-}$

Cesium fluoride, $\mathrm{CsF}_{\mathrm{s}}$

Cesium fluosilicate, $\mathrm{CS}_{2} \mathrm{SiF}_{6}$

Cesium gallum sulfate dodecahydrate, $\mathrm{C}_{\mathrm{S}} \mathrm{Ga}\left(\mathrm{SO}_{4}\right)_{2} \cdot 12 \mathrm{H}_{2} \mathrm{O}$

Cesium iodide, CsI iron sulfate dodecahydrate,

$\mathrm{CsFe}\left(\mathrm{SO}_{4}\right)_{2} \cdot 12 \mathrm{H}_{2} \mathrm{O}$

Cesium nitrate, $\mathrm{CsNO}_{3}$

Cesium perchlorate, $\mathrm{Cs}_{5} \mathrm{CiO}_{4}$, (orthorhombic) -

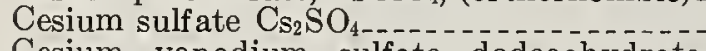

Cesium vanadium sulfate dodecahydrate,

$\mathrm{CsV}\left(\mathrm{SO}_{4}\right)_{2} \cdot 12 \mathrm{H}_{2} \mathrm{O}_{2} \ldots$

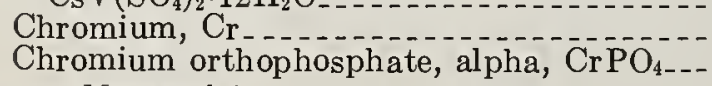

m-Monograph 25.

Indium oxide, $\mathrm{In}_{2} \mathrm{O}_{3-}$ 


\section{CUMULATIVE INDEX TO CIRCULAR 539, VOLUMES $1,2,3,4,5,6,7,8,9,10$,}

MONOGRAPH 25, SECTIONS 1, 2, AND $3^{5}$-Continued

Vol. or
sec.

Iodic acid, $\mathrm{HIO}_{3-}$

Iodine, $\mathrm{I}_{2-\ldots}$

Iridium, Ir . . .

Iron, alpha Fe

Iron arsenide, FeAs...................

Iron arsenide (loellingite), FeAs $\mathbf{s}_{2 \ldots}$

Iron sulfide (pyrite), $\mathrm{FeS}_{2} \ldots \ldots \ldots$

Lanthanum arsenate, $\mathrm{LaAsO}_{4} \ldots \ldots \ldots$

Lanthanum borate, $\mathrm{LaBO}_{3-}$

Lanthanum chloride, $\mathrm{LaCl}_{3} \ldots \ldots \ldots$

Lanthanum fluoride, $\mathrm{LaF}_{3} \ldots$

Lanthanum magnesium nitrate 24 -hydrate,

$\mathrm{La}_{2} \mathrm{Mg}_{3}\left(\mathrm{NO}_{3}\right)_{12} \cdot 24 \mathrm{H}_{2} \mathrm{O} \ldots$

Lanthanum niobium titanium oxide,

$\mathrm{LaNbTiO} 6 \ldots$

Lanthanum oxide, $\mathrm{La}_{2} \mathrm{O}_{3} \ldots$

Lead, $\mathrm{Pb}$

Lead bromide, $\mathrm{PbBr}_{2} \ldots \ldots$

Lead carbonate (cerrussite), $\mathrm{PbCO}_{3 \ldots} \ldots$

Lead chloride (cotunnite), $\mathrm{PbCl}_{2 \ldots} \ldots$

Lead formate, $\mathrm{Pb}\left(\mathrm{HCO}_{2}\right)_{2}$

Lead fluoride, alpha $\mathrm{PbF}_{2}$ (orthorhombic) -

Lead fluoride, beta $\mathrm{PbF}_{2}$ (cubic)

Lead (II), iodide, $\mathrm{PbI}_{2} \ldots$

Lead molybdate (wulfenite), $\mathrm{PbMoO}_{4} \ldots$

Lead monoxide (litharge), $\mathrm{PbO}$ (red) tetrag-

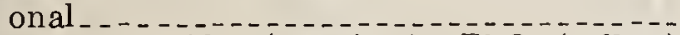

Lead monoxide (massicot), $\mathrm{PbO}$ (yellow) (orthorhombic)

Lead nitrate, $\mathrm{Pb}\left(\mathrm{NO}_{3}\right)_{2} \ldots$

Lead (II, III) oxide (minium), $\mathrm{Pb}_{3} \mathrm{O}_{4} \ldots$

Lead phos phate hydrate, $\mathrm{Pb}_{5}\left(\mathrm{PO}_{4}\right)_{3} \mathrm{OH}$

Lead selenide (clausthalite), $\mathrm{PbSe} . . . . .$.

Lead sulfate (anglesite), $\mathrm{PbSO}_{4-\ldots \ldots \ldots} \ldots$

Lead sulfide (galena), $\mathrm{PbS}$.

Lead titanate, $\mathrm{PbTiO}_{3} \ldots \ldots \ldots$

Lead tungstate (stolzite), $\mathrm{PbWO}_{4}$

Lithium arsenate, $\mathrm{Li}_{3} \mathrm{AsO}_{4} \ldots$

Lithium bromide, $\mathrm{LiBr}$

Lithium chloride, $\mathrm{LiCl} \ldots \ldots \ldots$

Lithium fluoride, $\mathrm{LiF}$

Lithium iodate, $\mathrm{LiIO}_{3 \ldots} \ldots \ldots$

Lithium molybdate, $\mathrm{Li}_{2} \mathrm{M}_{0} \mathrm{O}_{4}$, (trigonal) $\ldots$

Lithium oxide, $\mathrm{Li}_{2} \mathrm{O}$

Lithium nitrate, $\mathrm{LiNO} \mathrm{O}_{3}$

Lithium phosphate, low form, (lithiophosphate), $\mathrm{Li}_{3} \mathrm{PO}_{4}$

Lithium phosphate, high form, $\mathrm{Li}_{3} \mathrm{PO}_{4-\ldots}$

Lithium trimetaphosphate trihydrate,

$\mathrm{Li}_{3} \mathrm{P}_{3} \mathrm{O}_{9} \cdot 3 \mathrm{H}_{2} \mathrm{O}_{\ldots} \ldots$

Lithium tungstate, $\mathrm{Li}_{2} \mathrm{~W}_{4}$, (trigonal)

Lithium tungstate hemihydrate,

$\mathrm{Li}_{2} \mathrm{WO}_{4} \cdot 1 / 2 \mathrm{H}_{2} \mathrm{O}$

Lutetium gallium oxide $3: 5, \mathrm{Lu}_{3} \mathrm{Ga}_{2}\left(\mathrm{GaO}_{4}\right)_{3--}$

Lutetium manganite, $\mathrm{LuMnO}_{3}$

Lutetium oxide, $\mathrm{Lu}_{2} \mathrm{O}_{3} \ldots$

Magnesium, $\mathrm{Mg}$

Magnesium aluminum silicate (low-cordierite), $\mathrm{Mg}_{2} \mathrm{Al}_{4} \mathrm{Si}_{5} \mathrm{O}_{18}$ (or thorhombic) -...-

Magnesium aluminum silicate (high-cordierite), $\mathrm{Mg}_{2} \mathrm{Al}_{4} \mathrm{Si}_{5} \mathrm{O}_{18}$ (hexagonal)

Magnesium ammonium phosphate hexahydrate, (struvite), $\mathrm{MgNH}{ }_{4} \mathrm{PO}_{4} \cdot 6 \mathrm{H}_{2} \mathrm{O}_{-}$

Magnesium carbonate (magnesite), $\mathrm{MgCO}_{3}$

Magnesium chromite (picrochromite),

$\mathrm{MgCr}_{2} \mathrm{O}_{4}$
Magnesium fluoride (sellaite), $\mathrm{MgF}_{2} \ldots \ldots$

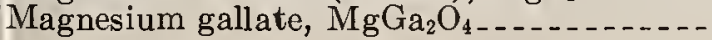
m-Monograph 20.

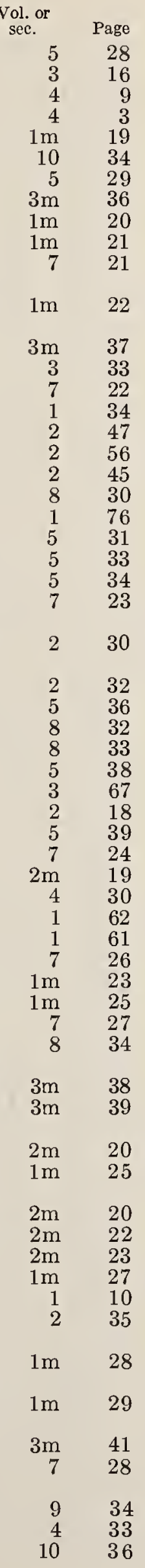

28

16

9

19

34

29

20

21

1

37

33

22

47

56

45

76

31

33

34
23

30

36

25

20

2

3

\section{0}

35

28

9

1
Magnesium germanate, $\mathrm{Mg}_{2} \mathrm{GeO}_{4}$ (cubic) ---
Magnesium germanate, $\mathrm{Mg}_{2} \mathrm{GeO}_{4}$ (ortho-

rhombic)
Magnesium hydroxide (brucite), $\mathrm{Mg}(\mathrm{OH})_{2--}$
Magnesium oxide (periclase), $\mathrm{MgO}$

Magnesium silicate, enstatite, $\mathrm{MgSiO}_{3} \ldots$

Magnesium silicate (forsterite), $\mathrm{Mg}_{2} \mathrm{SiO}_{4-\ldots}$

Magnesium silicate fluoride (norbergite), $\mathrm{Mg}_{2} \mathrm{SiO}_{4} \cdot \mathrm{MgF}_{2} \ldots$

Magnesium silicate fluoride (humite), $3 \mathrm{Mg}_{2} \mathrm{SiO}_{4} \cdot \mathrm{MgF}_{2}$
Magnesium sulfate heptahydrate (epsomite),

$\mathrm{MgSO}_{4} \cdot 7 \mathrm{H}_{2} \mathrm{O}$

Magnesium sulfide, $\mathrm{MgS}$

Magnesium tin, $\mathrm{Mg}_{2} \mathrm{Sn}$

Magnesium titanate (geikielite), $\mathrm{MgTiO}_{3-}$

Magnesium tungstate, $\mathrm{MgW} \mathrm{O} \mathrm{O}_{4}$
Manganese aluminate (galaxite), $\mathrm{MnAl}_{2} \mathrm{O}_{4-}$

Manganese(II) carbonate (rhodochrosite), $\mathrm{MnCO}_{3}$

Manganese ferrite (jacobsite), $\mathrm{MnF}{ }_{2} \mathrm{O}_{4}$

Manganese(II) oxide (manganosite), $\mathrm{MnO}$

Manganese(III) oxide (partridgeite), $\mathrm{Mn}_{2} \mathrm{O}_{3}$

Manganese selenide, MnSe.

Manganese sulfide (alabandite), alpha MnS

Manganese(II) tungstate (huebnerite), $\mathrm{MnWO}$

Mercury (I) bromide, $\mathrm{Hg}_{2} \mathrm{Br}_{2} \ldots, \mathrm{Hg}_{2} \mathrm{Cl}_{2} \ldots \ldots$
Mercury(I) chloride (calomel),

Mercury(II) chloride, $\mathrm{HgCl}_{2-}$

Mercury(II) cyanide, $\mathrm{Hg}(\mathrm{CN})_{2}$

Mercury(II) fluoride, $\mathbf{H g F}_{2}$

Mercury(I) iodide, $\mathrm{H}$ gI

Mercury(II) iodide, $\mathrm{HgI}_{2-}$

Mercury(II) oxide (montroydite), $\mathrm{HgO}_{-}$ (revised)

Vol. or

sec.

Mercury(II) selenide (tiemannite), HgSe...

Mercury(II) sulfide (cinnabar), HgS (hexagonal

Mercury(II) sulfide (metacinnabar), $\mathrm{HgS}$ (cubic)

Molybdenum, Mo

Molybdenum disulfide (molybdenite), MoS2

Molybdenum trioxide (molybdite), $\mathrm{MoO}_{3-\ldots}$

Neodymium borate, $\mathrm{NdBO}_{3}$

Neodymium chloride, $\mathrm{NdCl}_{3}$

Neodymium ethylsulfate nonahydrate, $\mathrm{Nd}\left[\left(\mathrm{C}_{2} \mathrm{H}_{5}\right) \mathrm{SO}_{4}\right]_{3} \cdot 9 \mathrm{H}_{2} \mathrm{O}$

Neodymium fluoride, $\mathrm{NdF}_{3}$

Neodymium gallium oxide $3: 5$,

$\mathrm{Nd}_{3} \mathrm{Ga}_{2}\left(\mathrm{GaO}_{4}\right)_{3-}$

Neodymium oxide, $\mathrm{Nd}_{2} \mathrm{O}_{3}$

Neodymium oxychloride, $\overline{\mathrm{NdOCl}}$

Nickel, $\mathrm{Ni}$

Nickel aluminate, $\mathrm{NiAl}_{2} \mathrm{O}_{4} \ldots$

Nickel arsenic 1:2 (rammelsbergite), $\mathrm{NiAs}_{2}$

Nickel arsenic sulfide (gersdorffite), NiAsS) -

Nickel (II) carbonate, $\mathrm{NiCO}_{3}$ (trigonal)

Nickel ferrite (trevorite), $\mathrm{NiFe}_{2} \mathrm{O}_{4}-\overline{-}-\overline{\mathrm{F}} \mathrm{H}_{2} \mathrm{O}$

Nickel gallate, $\mathrm{NiGa}_{2} \mathrm{O}_{4}$

Nickel germanate, $\mathrm{Ni}_{2} \mathrm{GeO}_{4} \ldots$

Nickel (II) oxide (bunsenite), $\mathrm{NiO}_{\ldots} \ldots . . .$.

Nickel sulfate, $\mathrm{NiSO}_{4 . \ldots} \ldots$

Nickel sulfate hexahydrate (retgersite), $\mathrm{NiSO}_{4} \cdot 6 \mathrm{H}_{2} \mathrm{O}$

Nickel sulfide, millerite, $\mathrm{NiS}$

Nickel tungstate, NiWO $\mathrm{N}_{4} \ldots$

Niobium silicide, $\mathrm{NbSi}_{2} \ldots$

Osmium, Os

Palladium, Pd

Palladium oxide, $\mathrm{Pd}{ }_{\mathrm{O}}$ 

MONOGRAPH 25, SECTIONS 1, 2, AND $3^{5}$-Continued

Vol. or

sec. Page

Platinum, Pt Potassium aluminum sulfate dodecahydrate

(alum), $\mathrm{KAl}\left(\mathrm{SO}_{4}\right)_{2} \cdot 12 \mathrm{H}_{2} \mathrm{O}_{-}$

Potassium borohydride, $\mathrm{KBH}_{4}$

Potassium bromate, $\mathrm{KBrO}_{3}$

Potassium bromide, $\mathrm{KBr}$

Potassium bromoplatinate, $\overline{\mathrm{K}}_{2} \mathrm{PtBr}_{6} \ldots \ldots$

Potassium bromoselenate, $\mathrm{K}_{2} \mathrm{SeBr}_{6 \ldots \ldots} \ldots$

Potassium chlorate, $\mathrm{KClO}_{3}$

Potassium chloride (sylvite), $\overline{\mathrm{K}} \mathrm{Cl}_{\ldots} \ldots \ldots$

Potassium chloroplatinate, $\mathrm{K}_{2} \mathrm{PtCl}_{6 \ldots} \ldots \ldots .$.

Potassium chlororhenate, $\mathrm{K}_{2} \mathrm{ReCl}_{6} \ldots \ldots . .$.

Potassium chlororuthenate (IV), $\mathrm{K}_{2} \mathrm{RuCl} \mathrm{Cl}_{6-}$

Potassium chlorostannate, $\mathrm{K}_{2} \mathrm{Sn} \mathrm{Cl}_{6 \ldots \ldots} \ldots \ldots$

Potassium chromium sulfate dodecahydrate,

$\mathrm{KCr}\left(\mathrm{SO}_{4}\right)_{2} \cdot 12 \mathrm{H}_{2} \mathrm{O}_{\ldots} \ldots \ldots$

Potassium cobaltinitrite, $\mathrm{K}_{3} \mathrm{Co}\left(\mathrm{NO}_{2}\right)_{6 \ldots \ldots} \ldots$

Potassium cyanate, $\mathrm{KCNO}$

Potassium cyanide, $\mathrm{KCN}$

Potassium dihydrogen arsenate, $\mathrm{KH}_{2} \mathrm{AsO}_{4}$ -

Potassium dihydrogen phosphate, $\mathrm{KH}_{2} \mathrm{PO}_{4}-$

Potassium fluogermanate, $\mathrm{K}_{2} \mathrm{GeF}_{6-}$

Potassium fluoplatinate, $\mathrm{K}_{2} \mathrm{PtF}_{6}$

Potassium fluoride, $\mathrm{KF}$

Potassium fluosilicate (hieratite), $\overline{\mathrm{K}}_{2} \mathrm{SiF}_{6-\ldots}$

Potassium fluotitanate, $\mathrm{K}_{2} \mathrm{TiF}_{6-\ldots} \ldots$

Potassium heptafluozirconate, $\mathrm{K}_{3} \mathrm{ZrF}_{7}$

Potassium hydroxy-chlororuthenate,

$\mathrm{K}_{4} \mathrm{Ru}_{2} \mathrm{Cl}_{10} \mathrm{O} \cdot \mathrm{H}_{2} \mathrm{O}$

Potassium iodide, $\overline{\mathrm{KI}}$

Potassium lithium sulfate, $\mathrm{K} \mathrm{LiSO}_{4} \ldots . .$.

Potassium metaperiodate, $\mathrm{KIO}_{4}$

Potassium nitrate (niter), $\mathrm{KNO}_{3} \ldots \ldots . . . .$.

Potassium nitroso chlororuthenate,

$\mathrm{K}_{2} \mathrm{RuCl}_{6} \mathrm{NO}$

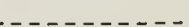

Potassium perchromate, $\mathrm{K}_{3} \mathrm{CrO}_{8}$

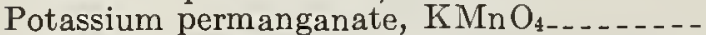

Potassium perrhenate, $\mathrm{KReO}_{4} \ldots$

Potassium phosphomolybdate, tetrahydrate,

$\mathrm{K}_{2} \mathrm{PO}_{4}\left(\mathrm{MoO}_{3}\right)_{12} \cdot 4 \mathrm{H}_{2} \mathrm{O}$

Potassium sulfate (arcanite), $\overline{\mathrm{K}}_{2} \mathrm{SO}_{4 \ldots \ldots} \ldots \ldots$

Potassium thioc ynate, KCNS

$\mathrm{K}_{2} \mathrm{Zn}_{2} \mathrm{~V}_{10} \mathrm{O}_{23} \cdot 16 \mathrm{H}_{2} \mathrm{O}$

Potassium zinc fluoride, $\mathrm{KZnF}_{3}$

Praseodymium chloride, $\mathrm{PrCl}_{3}$

Praseodymium fluoride, $\mathrm{PrF}_{3}$

Praseodymium oxychloride, PrOCl.

Rhenium, $\mathrm{Re}$

Rhodium, $\mathrm{Rh}$

$\mathrm{RbAl}\left(\mathrm{SO}_{4}\right)_{2} \cdot 12 \mathrm{H}_{2} \mathrm{O}_{\ldots} \ldots \ldots$

Rubidium bromate, $\mathrm{RbBrO}_{3}$

Rubidium bromide, $\mathrm{RbBr}$

Rubidium bromotellurate, $\mathrm{Rb}_{2} \mathrm{Te}_{\mathrm{Br}} \mathrm{Br}_{6} \ldots \ldots$

Rubidium chlorate, $\mathrm{RbClO}_{3} \ldots \ldots . . . . . .$.

Rubidium chloride, $\mathrm{RbCl}$.

Rubidium chloroplatinate, $\mathrm{Rb}_{2} \mathrm{PtCl}_{6} \ldots \ldots$

Rubidium chlorostannate, $\mathrm{Rb}_{2} \mathrm{Sn}_{\mathrm{Cl}} \mathrm{Cl}_{6} \ldots \ldots \ldots$

Rubidium chlorotellurate, $\mathrm{Rb}_{2} \mathrm{TeCl}_{0_{-} \ldots \ldots} \ldots$

Rubidium chromate, $\mathrm{Rb}_{2} \mathrm{CrO}_{4} \ldots \ldots . . . . .$.

Rubidium chromium sulfate dodecahydrate;

$\mathrm{RbCr}\left(\mathrm{SO}_{4}\right)_{2} \cdot 12 \mathrm{H}_{2} \mathrm{O}_{\ldots} \ldots \ldots$

Rubidium fluoplatinate, $\mathrm{Rb}_{2} \mathrm{PtF}_{0 .} \ldots \ldots . .$.

Rubidium fluosilicate, $\mathrm{Rb}_{2} \mathrm{SiF}_{6}$

Rubidium iodide, $\mathrm{RbI}$

Rubidium perchlorate, $\mathrm{RbClO}_{4}$

Rubidium periodate, $\mathrm{RbIO}_{4}$

Rubidium sulfate, $\mathrm{Rb}_{2} \mathrm{SO}_{4}$

Ruthenium, Ru

m-Monograph 25.
Samarium chloride, $\mathrm{SmCl}_{3}$

Samarium fluoride, $\mathrm{SmF}_{3}$

Samarium gallium oxide $3: 5, \mathrm{Sm}_{3} \mathrm{Ga}_{2}\left(\mathrm{GaO}_{4}\right)_{3-}$ Samarium ox ychloride, $\mathrm{SmOCl}$

Scandium oxide, $\mathrm{Sc}_{2} \mathrm{O}_{3}--\overline{\mathrm{S}}--$

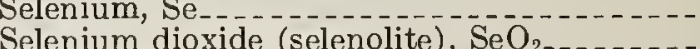

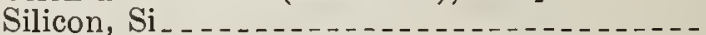

Silicon dioxide alpha or low quartz, $\mathrm{SiO}_{2}$ (hexagonal)

Silicon dioxide (alpha or low cristobalite), $\mathrm{SiO}_{2}$ (Revised) (tetragonal) - .......... Silicon dioxide (beta or high crisobalite), $\mathrm{SiO}_{2}$ (cubic)

Silver, Ag

Silver antimony telluride, AgSbTe

Silver arsenate, $\mathrm{Ag}_{3} \mathrm{AsO}_{4} \ldots \ldots$

Silver bromate, $\mathrm{AgBrO}_{3}$

Silver bromide (bromyrite), $\mathrm{AgBr}_{-}$

Silver carbonate, $\mathrm{Ag}_{2} \mathrm{CO}_{3} \ldots \ldots$

Silver chlorate, $\mathrm{AgClO}$

Silver chloride, (cerargyrite), $\mathrm{AgCl} \ldots \ldots$

Silver iodide (iodyrite), AgI (hexagonal)

Silver iodide, gamma, AgI (cubic) _......-

Silver metaperiodate, $\mathrm{AgIO}_{4} \ldots$

Silver molybdate, $\mathrm{Ag}_{2} \mathrm{MoO}_{4-\ldots} \ldots \ldots$

Silver nitrate, $\mathrm{AgNO}_{3}$

Silver nitrite, AgNO

Silver oxide, $\mathrm{Ag}_{2} \mathrm{O}$

Silver (II) ox ynitrate, $\mathrm{Ag}_{7} \mathrm{O}_{8} \mathrm{NO}_{3} \ldots \ldots$

Silver perrhenate, AgReO

Silver phosphate, $\mathrm{Ag}_{3} \mathrm{PO}_{4}$

Silver selenate, $\mathrm{Ag}_{2} \mathrm{SeO}_{4-}$

(1) - n

Silver sulfate, $\mathrm{Ag}_{2} \mathrm{SO}_{4} \ldots \ldots \ldots \ldots$

Silver sulfide (argentite), $\mathrm{Ag}_{2} \mathrm{~S} \ldots \ldots \ldots$

Sodium acid fluoride, $\mathrm{NaHF}_{2}$

Sodium borohydride, $\mathrm{NaBH}_{4}$

Sodium bromate, $\mathrm{NaBrO}_{3}$

Sodium bromide, $\mathrm{NaBr} \ldots \ldots \ldots$

Sodium carbonate monohydrate (thermonatrite), $\mathrm{Na}_{2} \mathrm{CO}_{3} \cdot \mathrm{H}_{2} \mathrm{O}$

Sodium chlorate, $\mathrm{NaClO}_{3} \ldots \ldots \ldots$

Sodium chloride (halite), $\mathrm{NaCl}_{-}$

Sodium cyanate, NaCNO

Sodium cyanide, $\mathrm{NaCN}$ (orthorhombic) at $6{ }^{\circ} \mathrm{C}$

Sodium fluoride (villiaumite), $\mathrm{NaF}$

Sodium iodate, $\mathrm{NaIO}_{3} \ldots \ldots \ldots$

Sodium iodide, $\mathrm{NaI}-$ droxy silicate, dravite,

$\mathrm{NaMg} \mathrm{Al}_{3} \mathrm{~B}_{3} \mathrm{Si}_{6} \mathrm{O}_{27}(\mathrm{OH})_{4}$

Sodium metaperiodate, $\mathrm{NaIO}_{4} \ldots$

Sodium molybdate, $\mathrm{Na}_{2} \mathrm{MoO}_{4}$

Sodium nitrate (soda-niter), $\mathrm{Na}_{\mathrm{N}} \mathrm{O}_{3 \ldots} \ldots$

Sodium nitrite, $\mathrm{NaNO}_{2} \ldots$

Sodium orthotungstate (VI) dihydrate, $\mathrm{Na}_{2} \mathrm{WO}_{4} \cdot 2 \mathrm{H}_{2} \mathrm{O}$

Sodium perchlorate, $\mathrm{NaClO}_{4}$ (orthorhombic)-

Sodium sulfate (thenardite), $\mathrm{Na}_{2} \mathrm{SO}_{4} \ldots$

Sodium sulfite, $\mathrm{Na}_{2} \mathrm{SO}_{3-}$

Sodium tetrametaphosphate tetrahydrate, alpha, $\mathrm{Na}_{4} \mathrm{P}_{4} \mathrm{O}_{12} \cdot 4 \mathrm{H}_{2} \mathrm{O}$ (monoclinic) ......

Sodium tetrametaphosphate tetrahydrate, beta, $\mathrm{Na}_{4} \mathrm{P}_{4} \mathrm{O}_{12} \cdot 4 \mathrm{H}_{2} \mathrm{O}$ (triclinic)

Sodium trimetaphosphate, $\mathrm{Na}_{3} \mathrm{P}_{3} \mathrm{O}_{3} \ldots \ldots$

Sodium trimetaphosphate monohydrate, $\mathrm{Na}_{3} \mathrm{P}_{3} \mathrm{O}_{9} \cdot \mathrm{H}_{2} \mathrm{O}_{-}$

Sodium tungstate, $\mathrm{Na}_{2} \mathrm{~W}_{3} \mathrm{O}_{4} \ldots$

Stannous fluoride, $\mathrm{SnF}_{2}$

Strontium arsenate, $\mathrm{Sr}_{3}\left(\mathrm{AsO}_{4}\right)_{2}$
Vol. or

sec.

$1 \mathrm{~m}$

$1 \mathrm{~m}$

$1 \mathrm{~m}$

$1 \mathrm{~m}$

3

8

5

2

3

10

1

$3 \mathrm{~m}$

5

5
4

$1 \mathrm{~m}$

7

8

9

9

7

5

$1 \mathrm{~m}$

4
8

5

$2 \mathrm{~m}$

7
10

5
9

5

$2 \mathrm{~m}$

Page

41 
Strontium 1:1 borate, $\mathrm{SrO} \cdot \mathrm{B}_{2} \mathrm{O}_{3-}$

Strontium bromide hexahydrate, $\mathrm{Sr} \mathrm{Br}_{2} \cdot 6 \mathrm{H}_{2} \mathrm{O}_{-}$ Strontium carbonate (strontianite), $\mathrm{SrCO}_{3--}$ Strontium chloride, $\mathrm{SrCl}_{2-}$

Strontium chloride hexahydrate, $\mathrm{SrCl}_{2} \cdot 6 \mathrm{H}_{2} \mathrm{O}_{-}$ Strontium fluoride, $\mathrm{SrF}_{2}$

Strontium formate, $\mathrm{Sr}\left(\mathrm{CHO}_{2}\right)_{2}$ Strontium formate dihydrate,

$\mathrm{Sr}\left(\mathrm{CHO}_{2}\right)_{2} \cdot 2 \mathrm{H}_{2} \mathrm{O}$ (orthorhombic)

Strontium iodide hexahydrate, $\mathrm{SrI}_{2} \cdot 6 \mathrm{H}_{2} \mathrm{O}_{---}$

Strontium molybdate, $\mathrm{SrMoO}_{4}$

Strontium nitrate, $\mathrm{Sr}\left(\mathrm{NO}_{3}\right)_{2 \ldots}$

Strontium oxide, $\mathrm{SrO}_{-}$

Strontium peroxide, $\mathrm{SrO}_{2}$

Strontium sulfate (celestite), $\mathrm{SrSO}_{4}$ Strontium sulfide, SrS.

Strontium titanate, $\mathrm{SrTiO}_{3}$ Strontium tungstate, $\mathrm{SrWO}_{4}$

Strontium zirconate, $\mathrm{SrZrO}_{3}$ Sulfamic acid, $\mathrm{NH}_{3} \mathrm{SO}_{3}$ Sulfur, S Tantalum, Ta Tantalum Silicide, $\mathrm{TaSi}_{2-}$

Tellurium, Te Tellurium (IV) oxide (paratellurite), $\mathrm{TeO}_{2}$
(tetragonal) Tellurium(IV) oxide, paratellurite, $\mathrm{TeO}_{2}$ (tetragonal) Tellurium(IV) oxide tellurite, $\mathrm{TeO}_{2}$ (ortho-
rhombic) Thombic) Thallium aluminum sulfate dodecahydrate, $\mathrm{TlAl}\left(\mathrm{SO}_{4}\right)_{2} \cdot 12 \mathrm{H}_{2} \mathrm{O}_{-}$

Thallium(I) arsenate, $\mathrm{Tl}_{3} \mathrm{AsO}_{4}$

Thallium (I) bromate, $\mathrm{Tl}_{\mathrm{BrO}} \ldots$

Thallium bromide, $\mathrm{TlBr}_{\mathrm{B}}$

Thallium(I) chlorate, $\mathrm{TlClO}_{3}$

Thallium(I) chloride, $\mathrm{TlCl}$

Thallium chloroplatinate, $\mathrm{Tl}_{2} \mathrm{PtCl}{ }_{6} \ldots$

Thallium chlorostannate, $\mathrm{Tl}_{2} \mathrm{Sn}_{\mathrm{Cl}} \mathrm{Cl}_{6} \ldots \ldots . .$.

Thallium chromate, $\mathrm{Tl}_{2} \mathrm{CrO}_{4-}$

Thallium chromium sulfate dodecahydrate, $\mathrm{TlCr}\left(\mathrm{SO}_{4}\right)_{2} \cdot 12 \mathrm{H}_{2} \mathrm{O}$

Thallium fluosilicate, $\mathrm{Tl}_{2} \mathrm{SiF}_{6}$ Thallium gallium sulfate dodecahydrate, $\mathrm{TlGa}\left(\mathrm{SO}_{4}\right)_{2} \cdot 12 \mathrm{H}_{2} \mathrm{O}_{-}$

Thallium(I) iodate, TiIO

Thallium(I) iodide, TII (orthorhombic)

Thallium(I) nitrate, $\mathrm{TlNO}_{3 \ldots} \ldots \ldots$

Thallium(III) oxide, $\mathrm{Tl}_{2} \mathrm{O}_{3} \ldots$

Thallium(I) perchlorate, $\mathrm{TIClO}_{4}$

Thallium(I) phosphate, $\mathrm{Tl}_{3} \mathrm{PO}_{4}$ Thallium(III) phosphate, TIPO Thallium(I) sulfate, $\mathrm{Tl}_{2} \mathrm{SO}_{4-}$

Thallium (I) thiocyanate, TICNS m-Monograph 25

\begin{tabular}{|c|c|}
\hline $\begin{array}{l}\text { Vol, or } \\
\text { səc. }\end{array}$ & Page \\
\hline $3 \mathrm{~m}$ & 53 \\
\hline 4 & 60 \\
\hline 3 & 56 \\
\hline 4 & 40 \\
\hline 4 & 58 \\
\hline 5 & 67 \\
\hline 8 & 55 \\
\hline 8 & 56 \\
\hline 8 & 58 \\
\hline 7 & 50 \\
\hline 1 & 80 \\
\hline 5 & 68 \\
\hline 6 & 52 \\
\hline 2 & 61 \\
\hline 7 & 52 \\
\hline 3 & 44 \\
\hline 7 & 53 \\
\hline 9 & 51 \\
\hline 7 & 54 \\
\hline 9 & 54 \\
\hline 1 & 29 \\
\hline 8 & 59 \\
\hline 1 & 26 \\
\hline 7 & 56 \\
\hline 10 & 55 \\
\hline 9 & 57 \\
\hline $3 \mathrm{~m}$ & 54 \\
\hline 6 & 53 \\
\hline $2 \mathrm{~m}$ & 37 \\
\hline 8 & 60 \\
\hline 7 & 57 \\
\hline 8 & 61 \\
\hline 4 & 51 \\
\hline 5 & 70 \\
\hline 6 & 54 \\
\hline $3 \mathrm{~m}$ & 54 \\
\hline 6 & 55 \\
\hline 6 & 56 \\
\hline 6 & 57 \\
\hline 8 & 62 \\
\hline 4 & 53 \\
\hline 6 & 58 \\
\hline 2 & 28 \\
\hline $2 \mathrm{~m}$ & 38 \\
\hline 7 & 58 \\
\hline 7 & 59 \\
\hline $\begin{array}{l}6 \\
8\end{array}$ & $\begin{array}{l}59 \\
63\end{array}$ \\
\hline
\end{tabular}

Thallium(I) tungstate, $\mathrm{Tl}_{2} \mathrm{WO}$

Thorium oxide (thorianite), Tho

Thulium arsenate, $\mathrm{TmAsO}_{4}$

Thulium sesquioxide, $\mathrm{Tm}_{2} \mathrm{O}_{3-}$

Tin, alpha, Sn (cubic)

Tin, beta, Sn (tetragonal)

Tin(IV) iodide, $\mathrm{SnI}_{4} \ldots \ldots \ldots$

Tin(II) oxide, SnO.

Tin (IV) oxide (cassiterite), $\mathrm{SnO}_{2 \ldots} \ldots \ldots$

Tin(II) telluride, SnTe.

Titanium, Ti...........................

Titanium dioxide (anatase), $\mathrm{TiO}_{2}$ (tetragonal)- Titanium dioxide, brookite, $\mathrm{TiO}_{2}$ (ortho
rhombic) Thombic)

Titanium(III) oxide, TiO ${ }_{1.515} \ldots$ Titanium silicide, $\mathrm{Ti}_{5} \mathrm{Si}_{3}$

Tungsten, $W$

Tungsten sulfide (tungstenite)

Uranium dioxide (uraninite), $\mathrm{UO}_{2} \ldots \ldots . .$. Urea, $\mathrm{CO}\left(\mathrm{NH}_{2}\right)_{2}$

Vanadium (V) oxide, $\mathrm{V}_{2} \mathrm{O}_{5-}$

Ytterbium gallium oxide $3: 5, \mathrm{Yb}$

Yttrium arsenate, $\mathrm{YAs}_{4}$

Yttrium gallium oxide $3: 5, \mathrm{Y}_{3} \mathrm{Ga}_{2}\left(\mathrm{GaO}_{4}\right)_{3 \ldots}$

Yttrium, oxide, $\mathrm{Y}_{2} \mathrm{O}_{3-}$

Yttrium oxychloride, $\mathrm{Y} \mathrm{OCl}_{\ldots} \ldots$

Yttrium phosphate (xenotime), $\mathrm{Y}_{\mathrm{PO}}$

Zinc, $\mathrm{Zn}_{2}$

Zinc borate, $\mathrm{ZnB}_{2} \mathrm{O}_{4}$

Zinc cyanide, $\mathrm{Zn}(\mathrm{CN})_{2 \ldots \ldots} \ldots$

Zinc, fluoride, $\mathrm{ZnF}_{2}$

Zinc fluosilicate hexahydrate, $\mathrm{ZnSiF}_{6} \cdot 6 \mathrm{H}_{2} \mathrm{O}_{-}^{-}$

Zinc germanate, $\mathrm{Zn}_{2} \mathrm{GeO}_{4}$

Zinc iodide, $\mathrm{ZnI}_{2} \ldots \ldots$

Zinc orthosilicate (willemite), $\mathrm{Zn}_{2} \mathrm{SiO}_{4-\ldots . .-}$

Zinc oxide (zincite), $\mathrm{ZnO}$

Zinc pyrosilicate hydrate, hemimorphite, $\mathrm{Zn}_{4}(\mathrm{OH})_{2} \mathrm{Si}_{2} \mathrm{O}_{7} \cdot \mathrm{H}_{2} \mathrm{O}$

Zinc selenide, ZnSe.

Zinc sulfate (zinkosite), $\mathrm{ZnSO}_{4} \ldots$

Zinc sulfate heptahydrate (goslarite), $\mathrm{ZnSO}_{4} \cdot 7 \mathrm{H}_{2} \mathrm{O}_{-}$

Zinc sulfide, (wurtzite), alpha $\mathrm{ZnS}$ (hexagonal)

Zinc sulfide, (sphalerite), beta ZnS (cubic) .-

Zinc telluride, ZnTe

Zinc tungstate, (sanmartinite), $\mathrm{Zn} \mathrm{WO}_{4-1}$

Zirconium, alpha, $\mathrm{Zr}$

Zirconium silicate zircon, $\mathrm{ZrSiO}$

Zirconium sulfate tetrahydrate,

$\mathrm{Zr}\left(\mathrm{SO}_{4}\right)_{2} \cdot 4 \mathrm{H}_{2} \mathrm{O}$
Vol, or

sec.

$1 \mathrm{~m}$

$3 \mathrm{~m}$

$3 \mathrm{~m}$

2

1

5

4

\section{CUMULATIVE MINERAL INDEX}

Alabandite $\mathrm{MnS}$

Alum, KAI $\left(\mathrm{SO}_{4}\right)_{2} \cdot 12 \mathrm{H}_{2} \mathrm{O}_{\ldots} \ldots$

Ammonia-niter, $\mathrm{NH}_{4} \mathrm{NO}_{3}$

Anatase, $\mathrm{TiO}_{2-}$

Andradite, $\mathrm{Ca}_{3} \mathrm{Fe}_{2} \mathrm{Si}_{3} \mathrm{O}_{12} \ldots$

$\begin{array}{rr}\begin{array}{c}\text { vol. or } \\ \text { sec. }\end{array} & \text { Page } \\ 4 & 11 \\ 6 & 36 \\ 7 & 4 \\ 1 & 46 \\ 9 & 22\end{array}$

Vol. or

Anglesite, $\mathrm{PbSO}_{4}$

Anhydrite, $\mathrm{CaSO}_{4} \ldots$

Aragonite, $\mathrm{CaCO}_{3}$

Argen tite, $\mathrm{Ag}_{2} \mathrm{~S}$

3

4

3
10

10
3

Page

67

65

53

51 
Arsenolite, $\mathrm{As}_{2} \mathrm{O}_{3}$

Aurostibite $\mathrm{AuSb}$

*Azurite, $\mathrm{Cu}_{3}(\mathrm{OH})_{2}\left(\mathrm{CO}_{3}\right)_{2 \ldots} \ldots$

Barite, $\mathrm{BaSO}$

Berlinite, $\mathrm{AlPO}_{4}$

*Beryl, $\mathrm{Be}_{3} \mathrm{Al}_{2}\left(\mathrm{SiO}_{3}\right)_{6}$

Bismite, (alpha) $\mathrm{Bi}_{2} \mathrm{O}_{3}$

Bismoclite, $\mathrm{BiOCl}$

Bismuthinite, $\mathrm{Bi}_{2} \mathrm{~S}_{3-}$

Böhmite, $\mathrm{Al}_{2} \mathrm{O}_{3} \cdot \mathrm{H}_{2} \mathrm{O}$

Bromellite, $\mathrm{BeO}$

Bromyrite, $\mathrm{AgBr}$

*Brookite, TiO

Brucite, $\mathrm{Mg}(\mathrm{OH})_{2}$

Bunsenite, $\mathrm{NiO}$

Calomel, $\mathrm{Hg}_{2} \mathrm{Cl}_{2}$

Cassiterite, $\mathrm{SnO}_{2}$

Celestite, $\mathrm{SrSO}_{4}$

Cerargyrite, $\mathrm{AgCl}$

Cerianite, $\mathrm{CeO}_{2}$

Cerrussite, $\mathrm{PbCO}_{3}$

Cervantite, $\mathrm{Sb}_{2} \mathrm{O}_{4}$

Chalcocyanite, $\mathrm{CuSO}_{4-}$

Chloraluminite, $\mathrm{AlCl}_{3}$ -

Chrysoberyl, $\mathrm{BeAl}_{2} \mathrm{O}_{4}$

Cinnabar, $\mathrm{HgS}$

* Claudetite, $\mathrm{As}_{2} \mathrm{O}_{3}$

Clausthalite, $\mathrm{PbSe}$

Cordierite, $\mathrm{Mg}_{2} \mathrm{Al}_{4} \mathrm{Si}_{5} \mathrm{O}_{18}$ (hexagonal) . . . . .

Corundum, $\mathrm{Al}_{2} \mathrm{O}_{3}$

Cotunnite, $\mathrm{PbCl}_{2}$

Covellite, $\mathrm{CuS}$

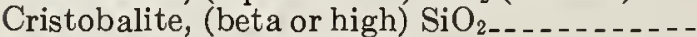

Cryptohalite, $\left(\mathrm{NH}_{4}\right)_{2} \mathrm{SiF}_{6}$

Cuprite, $\mathrm{Cu}_{2} \mathrm{O}$

*Diaspore, $\mathrm{Al}_{2} \mathrm{O}_{3} \cdot \mathrm{H}_{2} \overline{\mathrm{O}}$

* Dravite, $\mathrm{NaMg} \mathrm{Ml}_{0} \mathrm{~B}_{3} \mathrm{Si}_{27}$

*Enstatite, $\mathrm{MgSiO}_{3}$

Epsomite, $\mathrm{MgSO}_{4} \cdot 7 \mathrm{H}_{2} \mathrm{O}_{\ldots} \ldots \ldots$

Eschynite, $\mathrm{CeNbTiO}$

Ettringite, $\mathrm{Al}_{2} \mathrm{O}_{3} \cdot 6 \mathrm{CaO} \cdot 3 \mathrm{SO}_{3} \cdot 31 \mathrm{H}_{2} \mathrm{O}$

Fluoroapatite, $\mathrm{Ca}_{5} \mathrm{~F}\left(\mathrm{PO}_{4}\right)_{3}$

Fluorite, $\mathrm{CaF}_{2}$

Forsterite, $\mathrm{Mg}_{2} \mathrm{SiO}_{4}$

Galaxite, $\mathrm{MnAl}_{2} \mathrm{O}_{4 \ldots \ldots} \ldots$

Galena, $\mathrm{PbS}$

Gahnite, $\mathrm{ZnAl} \mathrm{O}_{4}$

Geikielite, $\mathrm{MgTiO}_{3}$

Gersdorffite, NiAsS

Goslarite, $\mathrm{ZnSO}_{4} \cdot 7 \mathrm{H}_{2} \mathrm{O}$

Greenockite, CdS

Halite, $\mathrm{NaCl}-\mathrm{Cl}_{-}$

Hieratite, $\mathrm{K}_{2} \mathrm{SiF}_{6}$

Huebnerite, $\mathrm{MnW} \mathrm{O}_{4}$

Humite, $3 \mathrm{Mg}_{2} \mathrm{SiO}_{4} \cdot \mathrm{MgF}_{2}$

Iodyrite, AgI

Jacobsite, $\mathrm{MnFe} \mathrm{O}_{4} \mathrm{O}_{4}$

Litharge, $\mathrm{PbO}$ (red)

Lithiophosphate, $\mathrm{Li}_{3} \mathrm{PO}_{4}$

Loellingite, FeAs?

Magnesite, $\mathrm{MgCO}_{3}$

Malachite, $\mathrm{Cu}_{2}(\mathrm{OH})_{2} \mathrm{CO}_{3}$

Manganosite, $\mathrm{MnO}$

Marshite, CuI

Mascagnite, $\left(\mathrm{NH}_{4}\right)_{2} \mathrm{SO}_{4}$ (revised)

* Natural mineral.

m-Monograph 25.
Massicot, $\mathrm{PbO}$ (yellow)

Matlockite, $\mathrm{PbFCl}$ -

*Millerite, NiS

Molybdenite, MoS

Montroydite, $\mathrm{HgO}$ (revised)

Mullite, $3 \mathrm{Al}_{2} \mathrm{O}_{3} \cdot 2 \mathrm{SiO}$

Nantokite, $\mathrm{CuCl}$

Niter, $\mathrm{KNO}_{3}$

Nitrobarite, $\mathrm{Ba}\left(\mathrm{NO}_{3}\right)_{2}$

Norbergite, $\mathrm{Mg}_{2} \mathrm{SiO}_{4} \cdot \mathrm{MgF}_{2}$

Oldhamite, $\mathrm{CaS}$

年

Otavite, $\mathrm{CdCO}_{3} \mathrm{CO}_{3}$

* Paratellurite, $\mathrm{TeO}_{2} \ldots \ldots$

Paratellurite, $\mathrm{TeO}_{2}$

Partridgeite, $\mathrm{Mn}_{2} \mathrm{O}_{3}$

*Phenacite, $\mathrm{Be}_{2} \mathrm{SiO}$

Picrochromite, $\mathrm{MgCr}_{2} \mathrm{O}_{4}$

Portlandite, $\mathrm{Ca}(\mathrm{OH})_{2}$

Powellite, $\mathrm{CaMoO}_{4}$

Pyrite, $\mathrm{FeS}_{2-}$

*Quartz, $\mathrm{SiO}_{2}$ (alpha or low)

Rammels bergite, NiAs 2

Retgersite, $\mathrm{NiSO}_{4} \cdot 6 \mathrm{H}_{2} \mathrm{O}$

Rutile, TiO

Safflorite, CoFeAs4

Sal-ammoniac, $\mathrm{NH}_{4} \mathrm{Cl}$

Sanmartinite, $\mathrm{ZnWO}_{4}$

* Scheelite, $\mathrm{CaWO}$

Selenolite, $\mathrm{SeO}_{2}$

Sellaite, $\mathrm{MgF}_{2}$

Senarmontite, $\mathrm{Sb}_{2} \mathrm{O}_{3}$

Skutterudite, $\mathrm{C}_{0} \mathrm{~A}_{\mathrm{S}_{3}}$

*Smithsonite, $\mathrm{Zn} \mathrm{CO}_{3}$

Soda-niter, $\mathrm{NaNO}$

Sphalerite, ZnS.

Spherocobaltite, $\mathrm{CoC} \mathrm{O}_{3}$

Stibnite, $\mathrm{Sb}_{2} \mathrm{~S}_{3}$

Strontianite, $\mathrm{SrCO}_{3}$

Struvite, $\mathrm{MgNH}_{4} \mathrm{PO}_{4} \cdot 6 \mathrm{H}_{2} \mathrm{O}$

Sylvite, $\mathrm{KCl}$

*Tellurite, $\mathrm{TeO}_{2} \ldots \ldots$

Tellurobismuthite, $\mathrm{Bi}_{2} \mathrm{Te}_{3} \ldots$

Tenorite, $\mathrm{CuO}$

Teschemacherite, $\mathrm{NH}_{4} \mathrm{H} \mathrm{CO}_{3}$

Teschermigite, $\mathrm{NH}_{4} \mathrm{Al}\left(\mathrm{SO}_{4}\right)_{2} \cdot 12 \mathrm{H}_{2} \mathrm{O}_{\ldots} \ldots \ldots$.

Thenardite, $\mathrm{Na}_{2} \mathrm{SO}_{4}$

Thermonatrite, $\mathrm{Na}_{2} \mathrm{CO}_{3} \cdot \mathrm{H}_{2} \mathrm{O}_{\ldots} \ldots$

Thorianite, $\mathrm{ThO}_{2}$

Tiemannite, $\mathrm{HgSe}$

*Topaz, $\mathrm{Al}_{2} \mathrm{SiO}_{4}(\mathrm{~F}, \mathrm{O} \mathrm{H})$

Trevorite, $\mathrm{NiFe}_{2} \mathrm{O}$

Tungstenite, $\mathrm{WS}_{2}$

Uraninite, $\mathrm{UO}_{2}$

Uvarovite, $\mathrm{Ca}_{3} \mathrm{Cr}_{2}\left(\mathrm{SiO}_{4}\right)_{3}$

*Valentinite, $\mathrm{Sb}_{2} \mathrm{O}_{3} \ldots \ldots$

Villiaumite, $\mathrm{NaF}$.

Willemite, $\mathrm{Zn}_{2} \mathrm{SiO}_{4-1}$

Witherite, $\mathrm{BaCO}_{3}$

Wulfenite, $\mathrm{PbMoO}_{4} \ldots$

Wurtzite, ZnS

Xenotime, $\mathrm{YPO}_{4} \ldots \ldots$

Zincite, $\mathrm{ZnO}_{\mathrm{O}}$

Zinkosite, $\mathrm{ZnSO}_{4}$

*Zircon, $\mathrm{ZrSiO}_{4}$
Vol. or

sec.

$1 \mathrm{~m}$

Page 

